Portland State University

PDXScholar

Dissertations and Theses

Dissertations and Theses

Spring 6-5-2019

\title{
Strength Tuned Steel Eccentric Braced Frames
}

Hosam Abdullah Al-Azzawi

Portland State University

Follow this and additional works at: https://pdxscholar.library.pdx.edu/open_access_etds

Part of the Civil and Environmental Engineering Commons Let us know how access to this document benefits you.

\section{Recommended Citation}

Al-Azzawi, Hosam Abdullah, "Strength Tuned Steel Eccentric Braced Frames" (2019). Dissertations and Theses. Paper 4981.

https://doi.org/10.15760/etd.6857

This Dissertation is brought to you for free and open access. It has been accepted for inclusion in Dissertations and Theses by an authorized administrator of PDXScholar. Please contact us if we can make this document more accessible: pdxscholar@pdx.edu. 
Strength Tuned Steel Eccentric Braced Frames

by

Hosam Abdullah Al-Azzawi

A dissertation submitted in partial fulfilment of the requirements for the degree of

\title{
Doctor of Philosophy \\ in
}

Civil and Environmental Engineering

\author{
Dissertation Committee: \\ Peter Dusicka, Chair \\ Franz Rad \\ Thomas Schumacher \\ Hormoz Zareh
}

Portland State University

2019 
(C) 2019 Hosam Abdullah Al-Azzawi 


\begin{abstract}
The primary component in eccentrically braced frames $(\mathrm{EBF})$ is the link as its plastic strength controls the design of the frame as well as the entire building within which it is installed. EBFs are the first part of building design and every other component is sized based on the forces developed in the link. Oversized link elements lead to the use of unnecessary materials and can increase construction costs. Additionally, the advantages of using a continuous member of the same depth for both the link and the controller beam (in terms of the cost and the time) motivates researchers to find a way to control the link strength in conventional EBFs. Previous studies on the link-to-column connections in EBF have shown that the links are likely to fail before reaching the required rotation due to fractures at low drift level. Moreover, improving the strength of the links in EBF depends primarily on their ability to achieve target inelastic deformation and to provide high ductility during earthquakes. Therefore, in this study, the concept of tuned link strength properties in EBF, T-EBF, is experimentally introduced as a solution to improve the performance of the link in conventional EBF by cutting out an opening in the link web. Furthermore, a new brace-to-link connection is proposed to bolt the brace member with the link in contrast to the conventional method of welding them. This new idea in continuous beam design was investigated to verify the stability of the tuned eccentrically braced frame, either welded or bolted, with a bracing member. A total of four full-scale cyclic tests were conducted to study the ability of T-EBF to achieve inelastic deformation. The specimens have two different cross sections: W18x76 and W16x67, two different sections where the brace was welded to the link, and two other
\end{abstract}


specimens at different sections where the brace was bolted to the link were examined. The experimental results indicate that the link in T-EBF can achieve high rotation, exceeding $0.15 \mathrm{rad}$, and an overstrength factor equal to 1.5. Failure involved included web buckling at very high rotation. The T-EBF displayed a very good, non-replaceable ductile link. The experiments were followed by an isotropic kinematic-combined hardening model in the finite element analyses (FEA). The FEA analysis is developed to predict the effect of web opening configuration on the local section stresses and strains and global characteristics of the frame. FEA exhibits good agreement with the experimental results and can capture the inelastic buckling behavior of the sections. The link configuration parameters of the T-EBF were studied extensively on a W18x76 shear link subjected to the 2016 AISC seismic design provisions loading protocol (ANSI/AISC 341-16, 2016). The parametric study also included the performance of a range of wide flange sections. The analysis shows that the reduced web section has effect on the plastic strain in which low plastic strain observed near ends and connections and high at the center of the web. Results also demonstrate that if the shear link is appropriately sized with web opening and intermediate web stiffeners provided, an excellent shear link with high ductility under cyclic loads can be obtained. Changing the configuration of the opening cutout also had a significant effect on reducing the transition zone cracks. 


\section{Dedication}

To my parents, my sisters and my brothers

To my wife

To my children 


\section{Acknowledgments}

First and foremost, I would like to thank God, Allah Almighty, for helping me and giving me the faith and strength to complete this dissertation. Many thanks to my advisor Dr. Peter Dusicka for his encouragement and thoughtful guidance during my studies. This work would not be possible without his support, patience and continuous advice. I also would like to extend my appreciation to my dissertation committee members, Dr. Franz Rad, Dr. Hormoz Zareh and Dr. Thomas Schumacher for their comments and review of this research.

Special thanks to the Higher Committee for Educational Development in Iraq for sponsoring my $\mathrm{PhD}$ program and partially funding this work. It is also grateful to my home university in Iraq, Tikrit University to support of leave of absence for several years. My gratitude goes to my friends and colleagues Wisam Aules, Anwer Mohammed, Qusay Aqooly, Anas Yosefani, Yasir Saeed, Salih Mahmood, Ilya Palknikov, and Aaron Smith for helping me with my experimental works.

My deep gratitude to my parents, two brothers, and three sisters for their help and supports. Finally, I wish to thank my wife, two sons, Saif and Suhaib, and daughter, Sarah, for their loves, patience and encouragement. 


\section{Table of Contents}

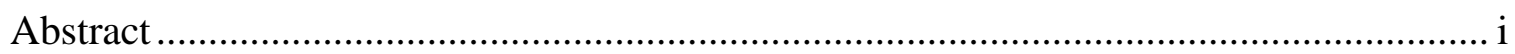

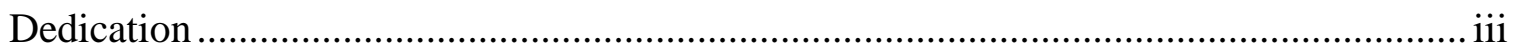

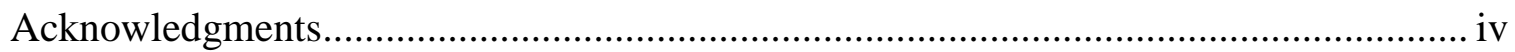

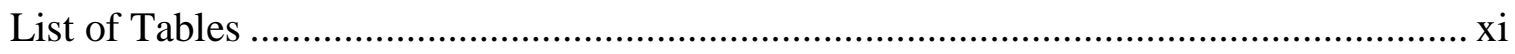

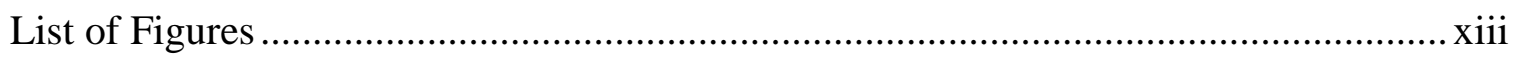

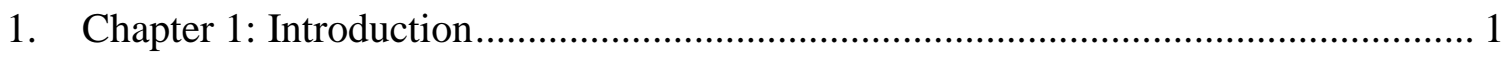

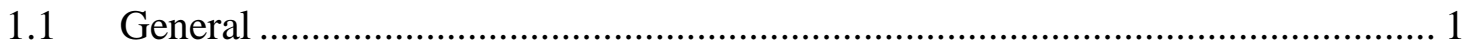

1.2 Eccentrically Braced Frames Damages and Repairs............................................ 4

1.1.1 Link Damages ................................................................................... 4

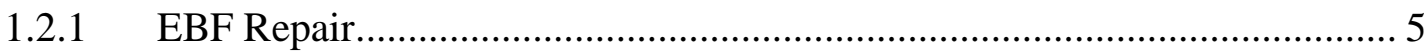

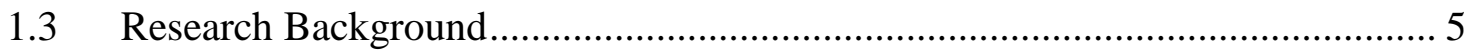

1.3.1 Failure Observed from Researchers ............................................................ 11

1.3.2 Failure Observed from Real Practice........................................................ 12

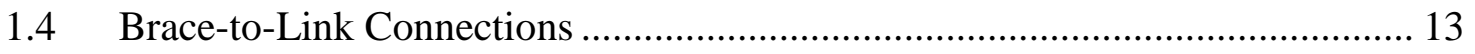

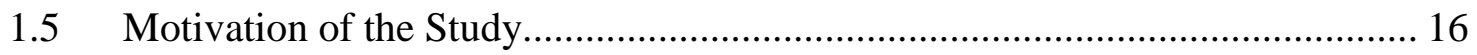

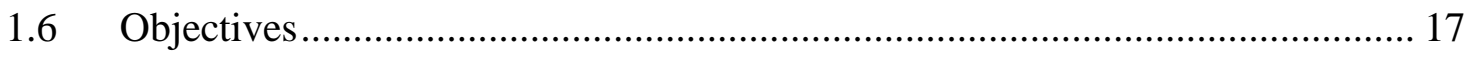

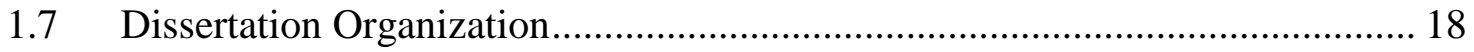


2 Chapter 2: Experimental Specimens and Test Setup.......................................... 20

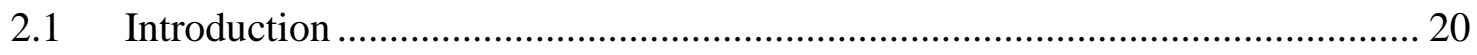

2.2 EBF Design Requirements ....................................................................... 20

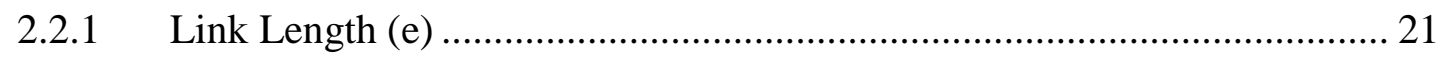

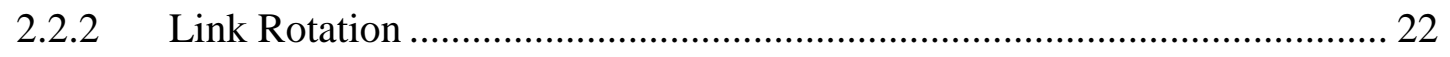

2.2.3 Forces in Links and End Moments ..................................................... 24

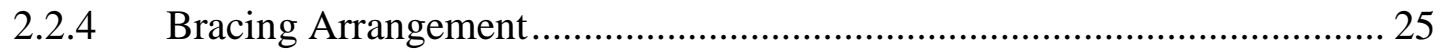

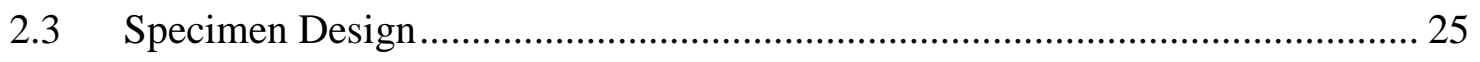

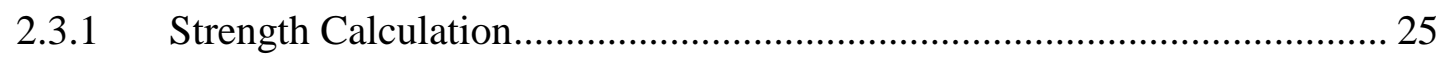

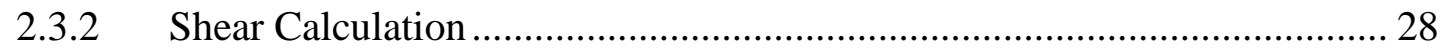

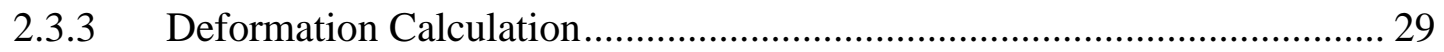

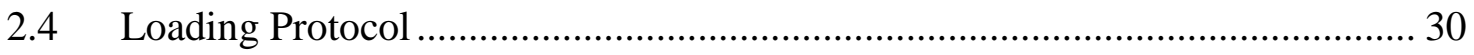

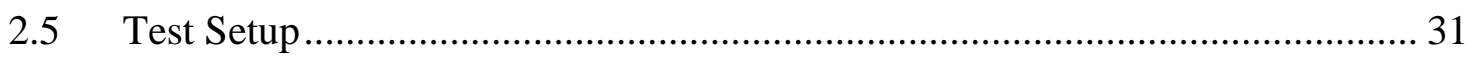

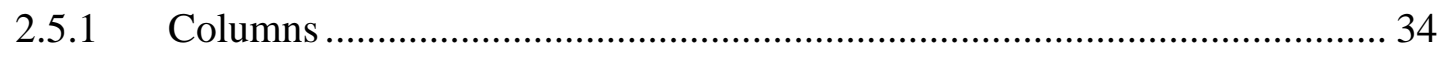

2.6 Test Phases and Specimens Description ................................................. 36

2.6.1 Phase One: T-EBF, Welded Diagonal Brace Connection ........................ 36

2.6.2 Phase Two: T-EBF, Bolted Diagonal Brace Connection........................... 38

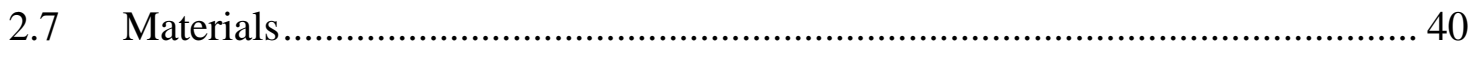

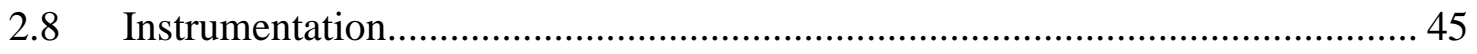




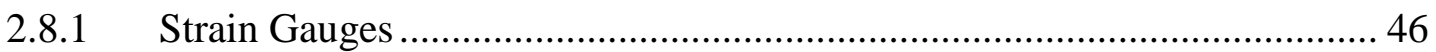

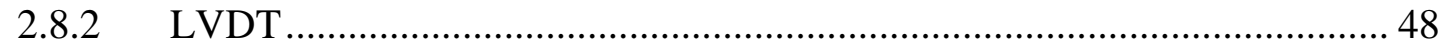

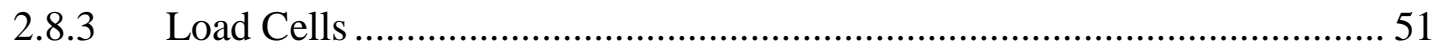

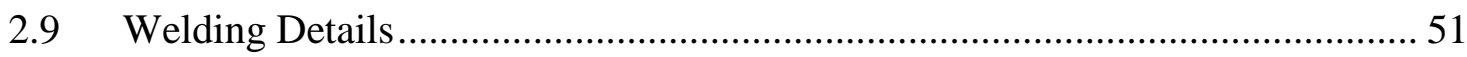

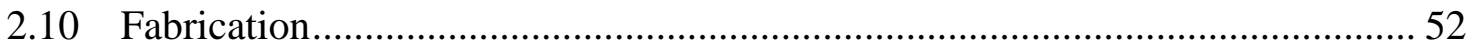

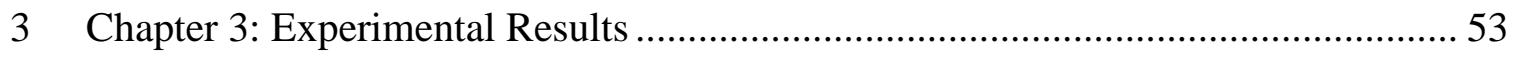

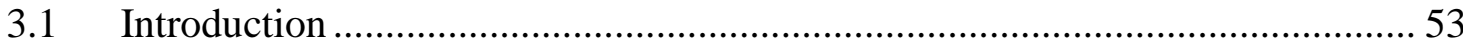

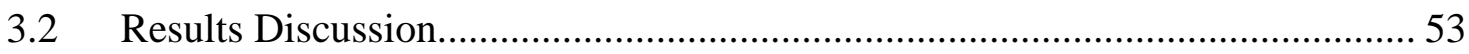

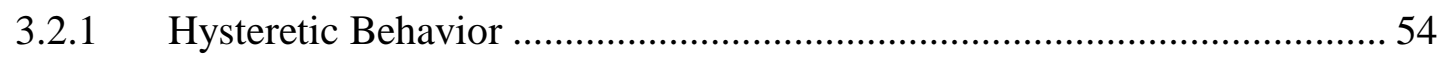

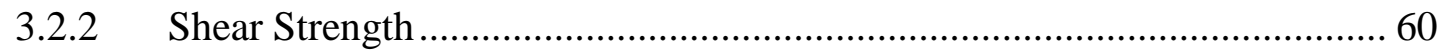

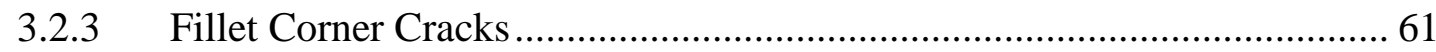

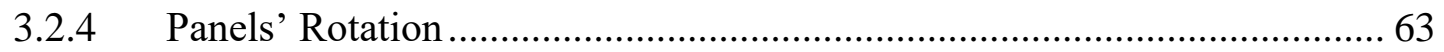

3.2.5 Shear Strain Discuss (Welded Brace) …………........................................ 70

3.2.6 Strain Development (Welded Brace) ………………….......................... 71

3.3 Compare strains for both welded and bolted brace ......................................... 75

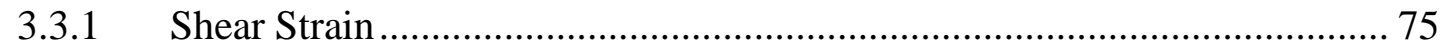

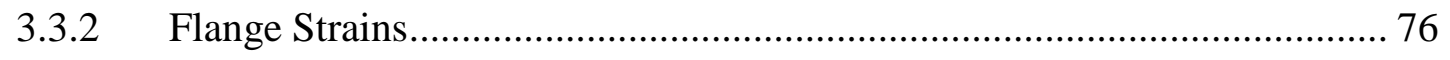

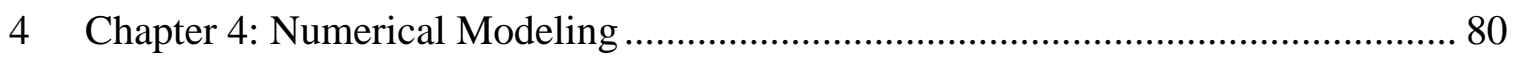

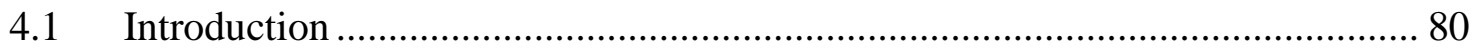


4.1.1 General Notes of the Model.....

4.2 Model Definition ................................................................................. 81

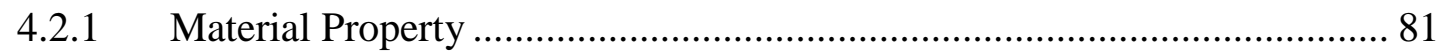

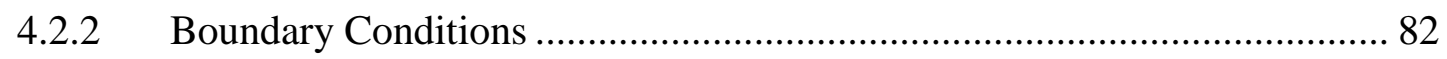

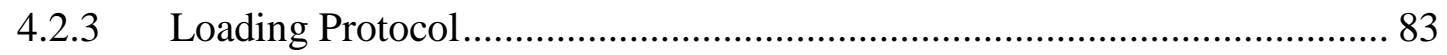

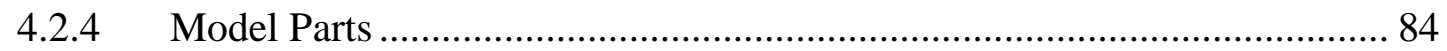

4.2.5 Partitions and Contact Surfaces ......................................................... 84

4.3 Element Types and Mesh ........................................................................ 84

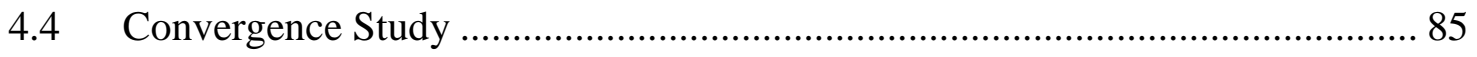

4.5 Validation of Experimental and Numerical Results................................... 89

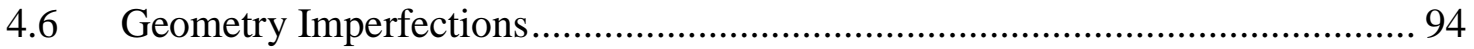

4.7 Bolted Brace to Link Simulation Using Finite Element Method ...................... 97

5 Chapter 5: Parametric Study on Key Dimensions of T-EBF................................ 103

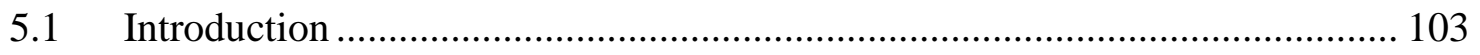

5.2 Control over EBF Strength................................................................. 104

5.3 Parameters Used in Design of T-EBF ...................................................... 105

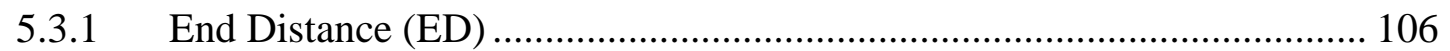

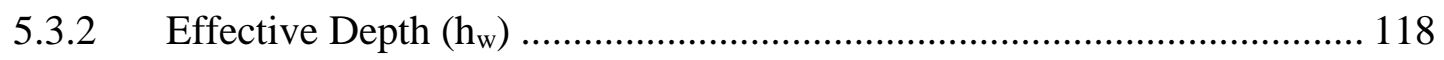

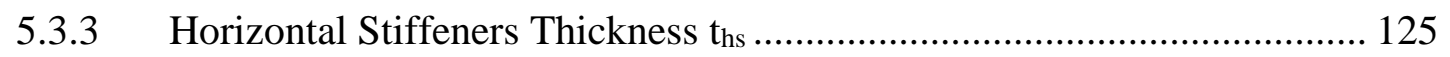


5.3.4 Transition Zone Radius ................................................................... 127

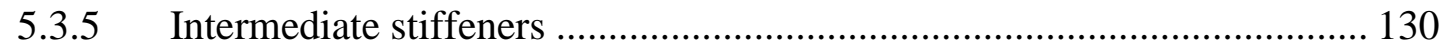

5.3.6 Tuned Common Sections ..................................................................... 134

5.4 Beam Outside the link ......................................................................... 139

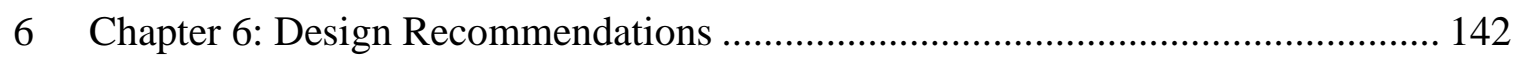

6.1 Design Loads .................................................................................... 142

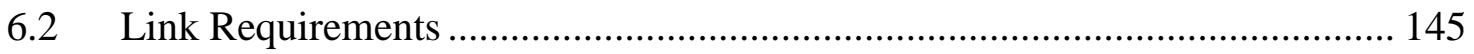

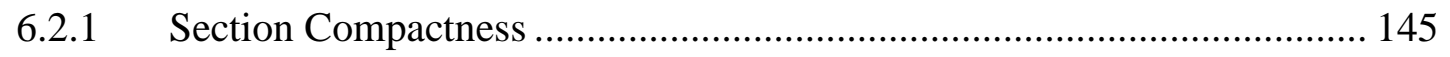

6.2.2 Link Rotation ............................................................................ 145

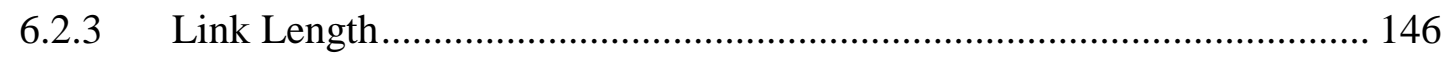

6.3 Link Design ..................................................................................... 146

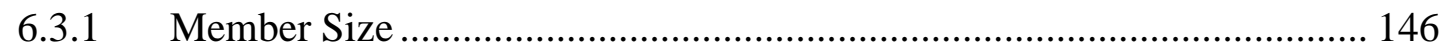

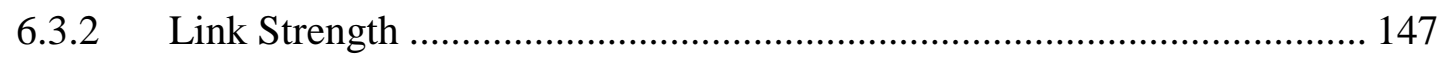

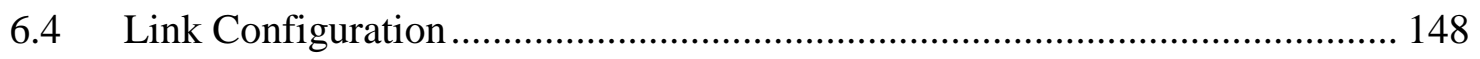

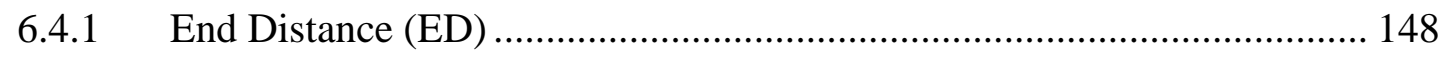

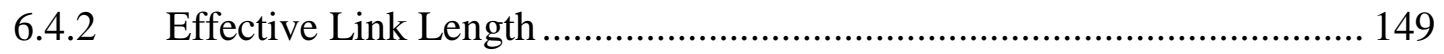

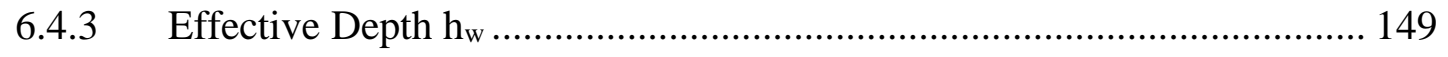

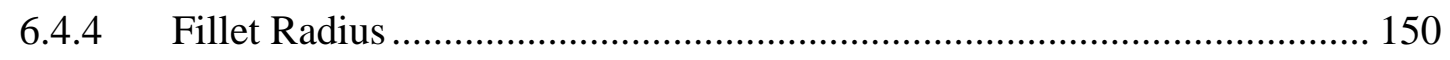

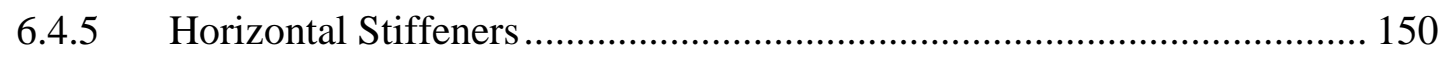




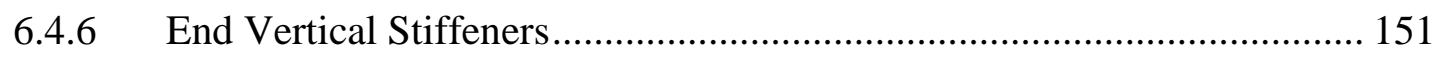

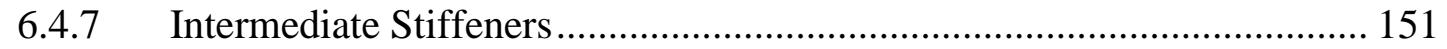

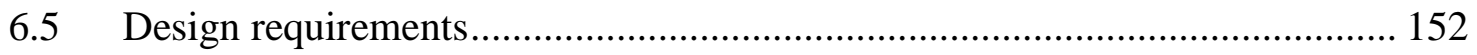

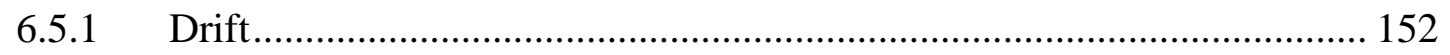

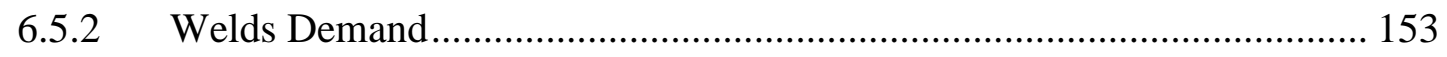

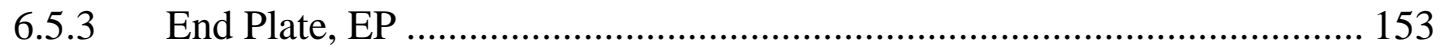

6.5.4 Brace-link Connection Design Requirement .......................................... 154

6.5.5 Beam Outside the Link Design ............................................................. 154

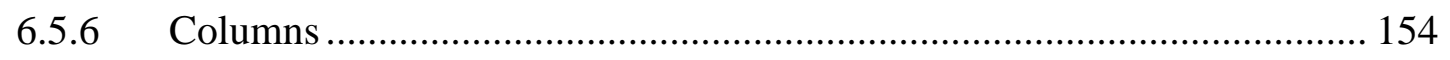

$7 \quad$ Chapter 7: Conclusions and Recommendations .................................................. 156

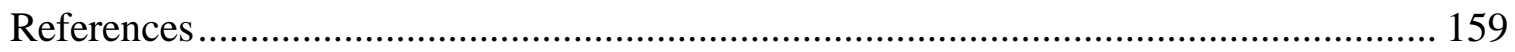

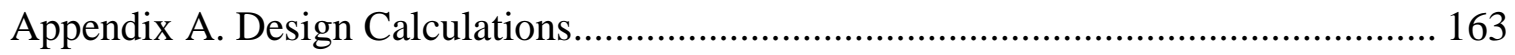

Appendix B. Model Parameters ............................................................................. 185

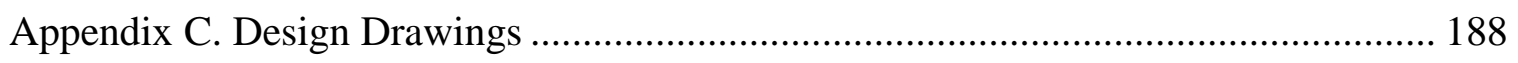

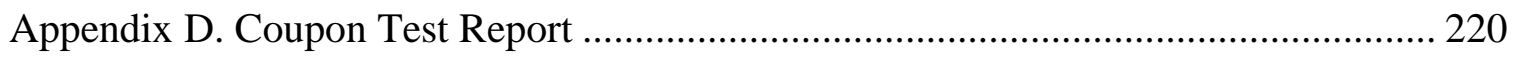




\section{List of Tables}

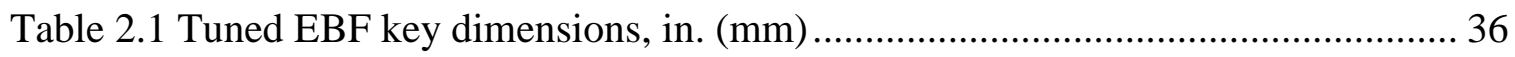

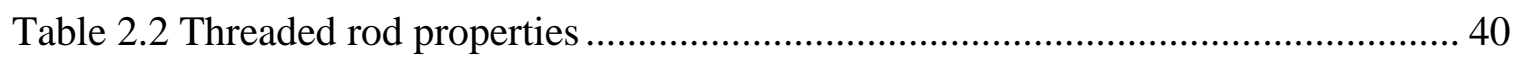

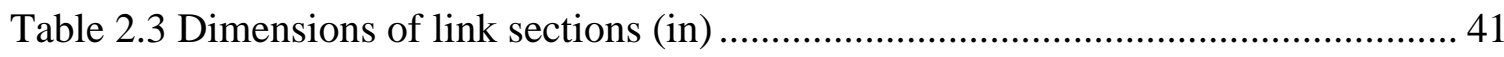

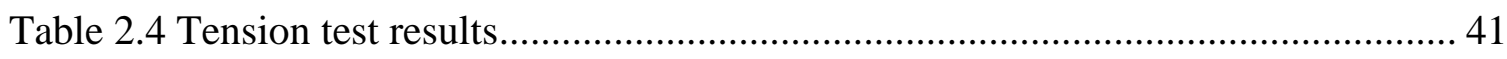

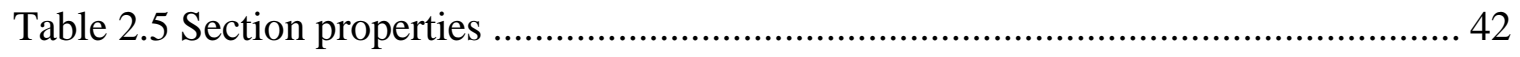

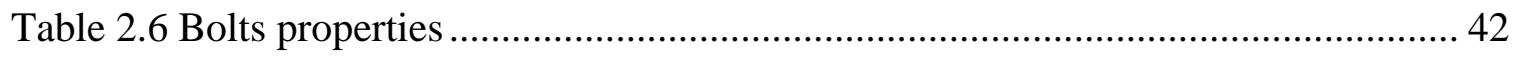

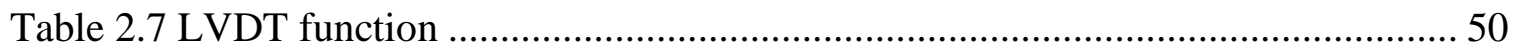

Table 3.1 Nominal design properties and section dimensions......................................... 60

Table 3.2 Maximum shear strength for all specimens ................................................... 60

Table 3.3 Displacement deformations of three panels translated from the panels' rotations

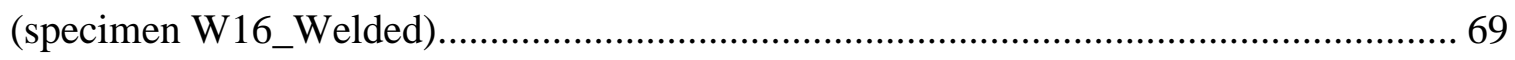

Table 3.4 Displacement deformations of three panels (specimens W18_Welded) .......... 69

Table 4.1 Combined Isotropic\& Kinematic material parameters used in FEA Volynkin

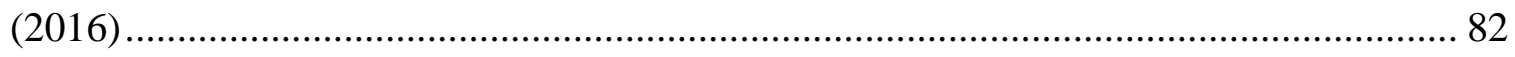

Table 4.2 Summary of contribution percentage of effective panel to the total link rotation

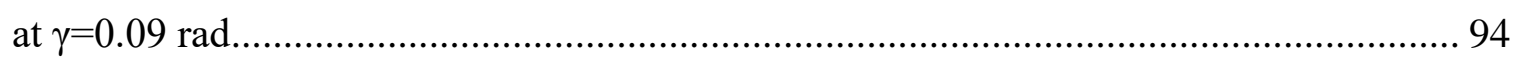

Table 4.3 Simple bilinear steel material used in FEA (Imam, 2015) ............................... 99

Table 5.1 Tuned section W18x76 properties, ED Models .............................................. 108

Table 5.2 PEEQ distributions of the models, ED models............................................... 114

Table 5.3 PEEQ for various cases of ED under cyclic loading at $\gamma=0.09 \mathrm{rad} . . . \ldots \ldots \ldots \ldots . . . .116$

Table 5.4 hw Parameter models, dimensions in inches ............................................. 118 
Table 5.5 Distribution of PEEQ within the links ..................................................... 122

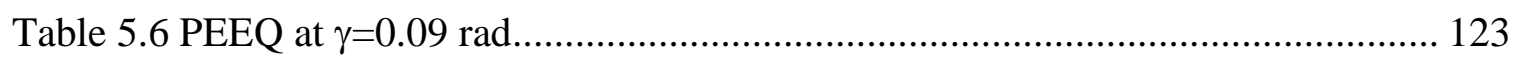

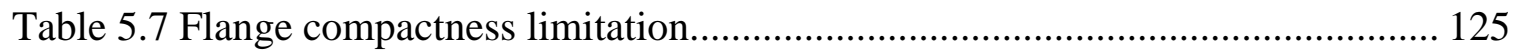

Table 5.8 PEEQ at transition zone radius (at rotation $0.09 \mathrm{rad}$ ) ................................ 129

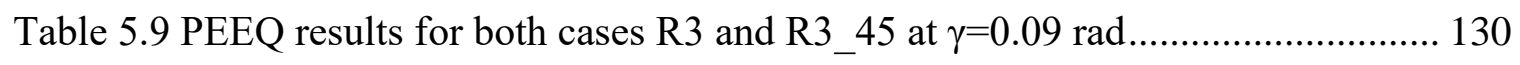

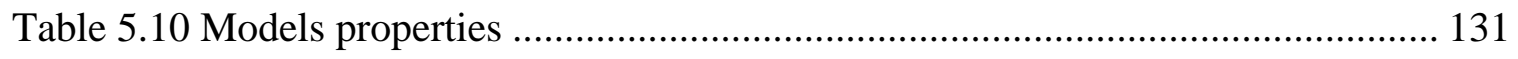

Table 5.11 Common sections' properties (in.)..................................................... 135

Table 5.12 Tuned Common sections' properties (in.) …....................................... 135

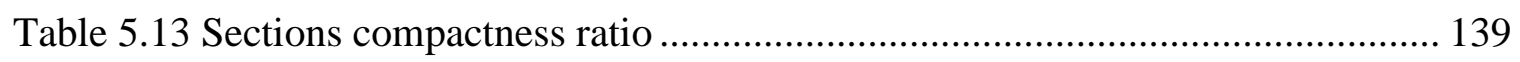




\section{List of Figures}

Figure 1.1 Three types of frames described above .................................................... 1

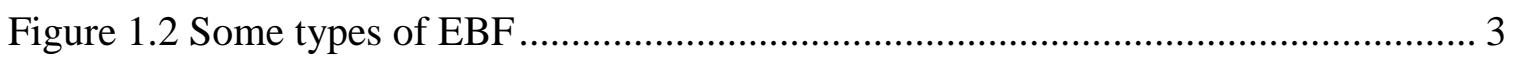

Figure 1.3 Test setup for bolted end plate replaceable links (Dusicka and Lewis,2010) ... 7

Figure 1.4 Fractures observed in some EBF links during tests ................................. 12

Figure 1.5 Various types of earthquake damages appeared in EBF (Clifton, et al. 2011) 13

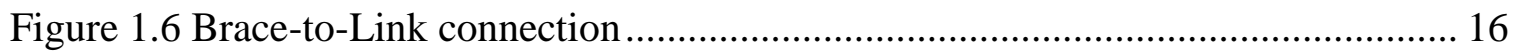

Figure 2.1 Link types related to the link rotation and length...................................... 22

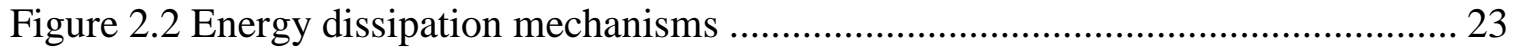

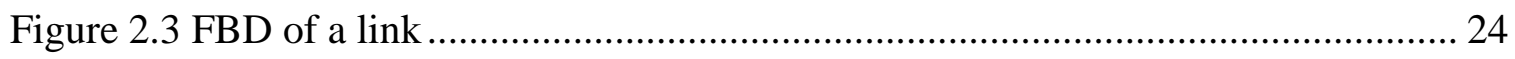

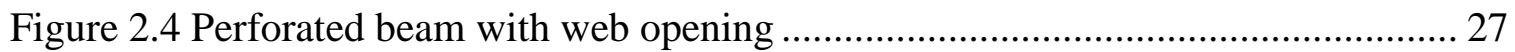

Figure 2.5 Link shear force in D-Braced EBF .................................................... 28

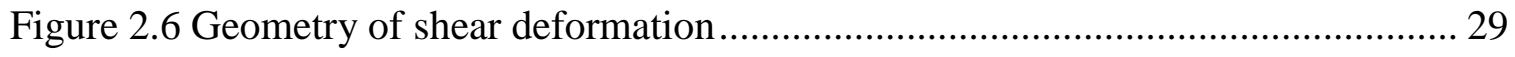

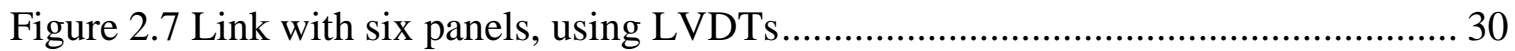

Figure 2.8 Loading protocol (AISC Seismic Design Provision) .................................. 31

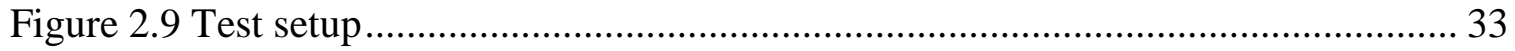

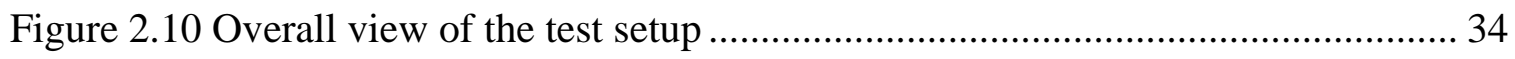

Figure 2.11 Columns details with used bolts and endplates locations........................... 35

Figure 2.12 Configuration of first specimen .......................................................... 37

Figure 2.13 Configuration of second specimen .................................................. 38

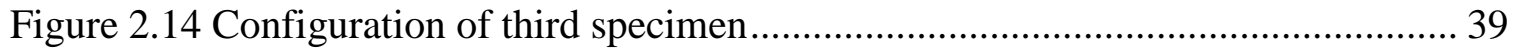

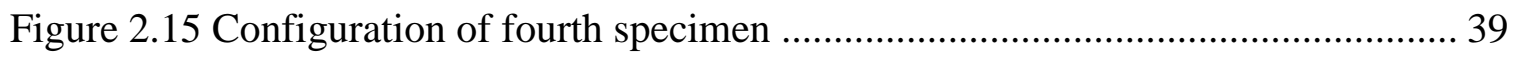




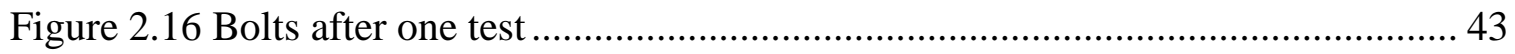

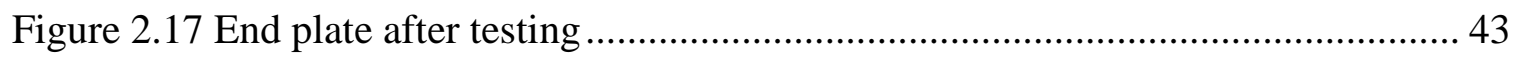

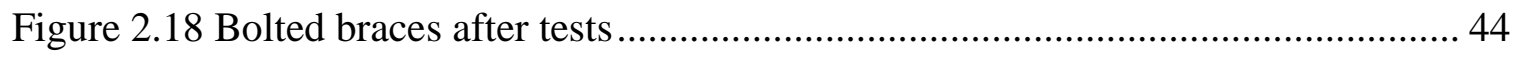

Figure 2.19 Instrumentations layout used on the EBF.......................................... 45

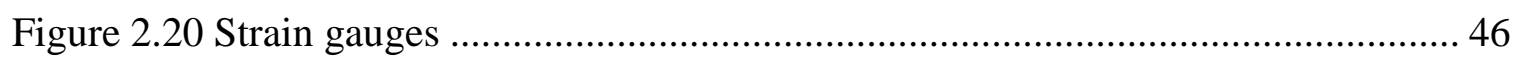

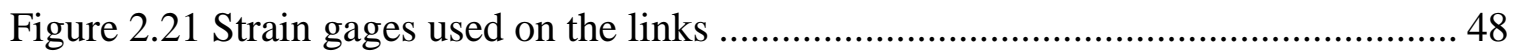

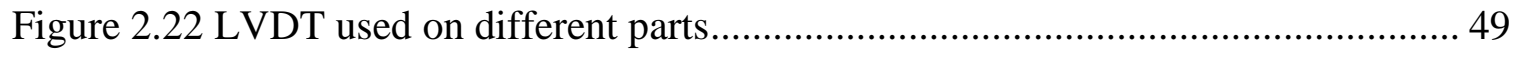

Figure 2.23 Load cell installed on the link ........................................................... 51

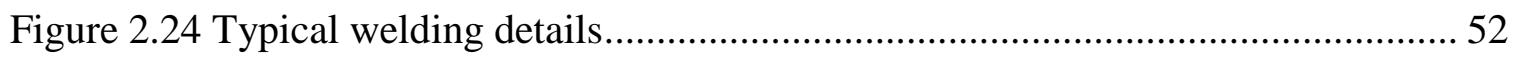

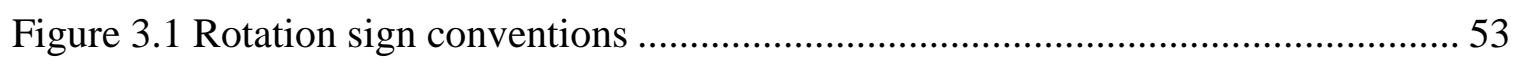

Figure 3.2 Comparison link shear force versus rotation hysteresis curve for both sections.

(n)

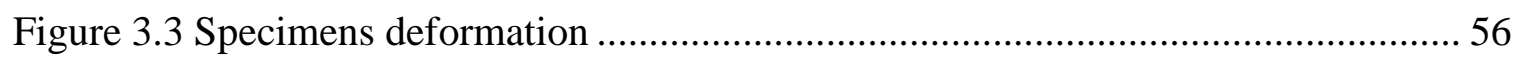

Figure 3.4 Specimens deformation (W16x67 Welded brace) .................................... 58

Figure 3.5 Specimens deformation (W16x67 Bolted brace) ..................................... 59

Figure 3.6 Opening corner cracks in three specimens at the north and south side .......... 62

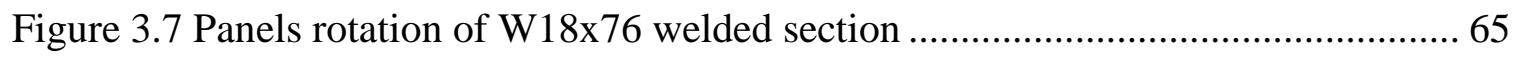

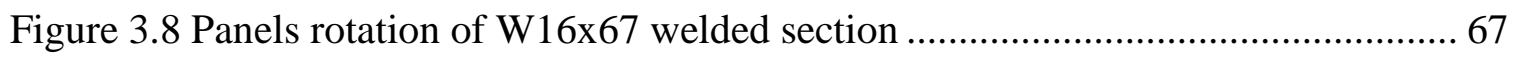

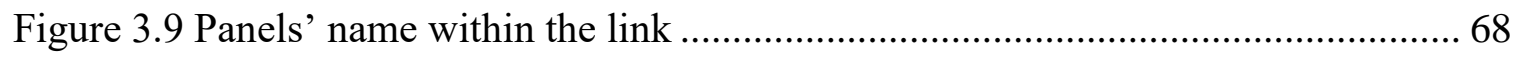

Figure 3.10 Contribution of the effective panel rotation to the total link rotation........... 70

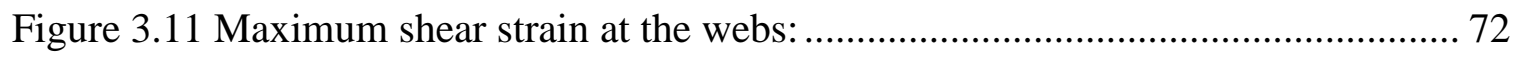

Figure 3.12 Strain at the flanges of W18 welded brace ......................................... 73 
Figure 3.13 Strain at the flanges of W16 welded brace 74

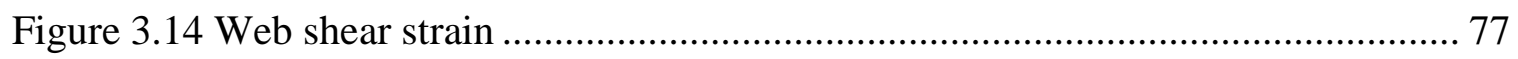

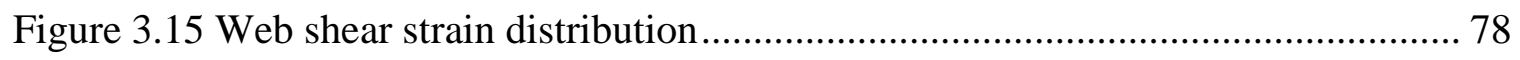

Figure 3.16 Middle flange strain distribution at the opening edge ............................... 79

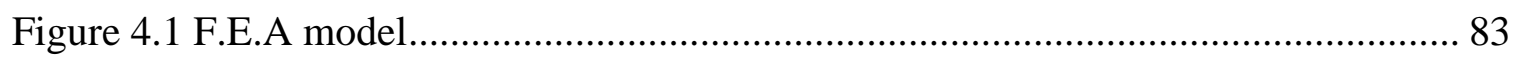

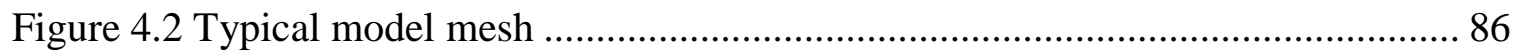

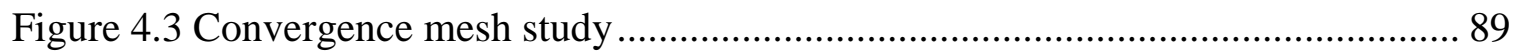

Figure 4.4 Link rotation-shear forces model results compared with the experimental data

91

Figure 4.5 Links deformation for FEA vs experiments ........................................ 93

Figure 4.6 Comparing end panels' rotations for experimental and numerical analysis

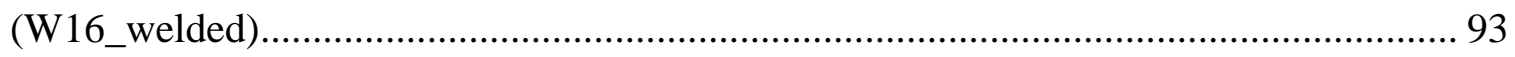

Figure 4.7 Effects of initial geometrical imperfection.......................................... 97

Figure 4.8 EBF described in the Christchurch Hospital Garage (Imani et al. 2015,

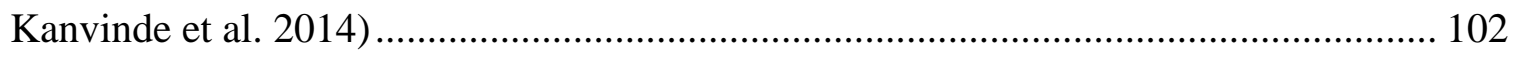

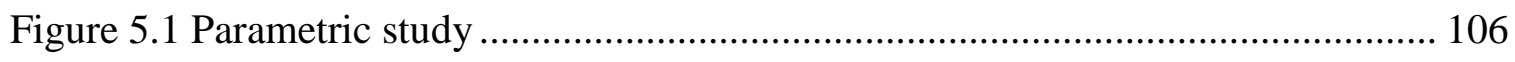

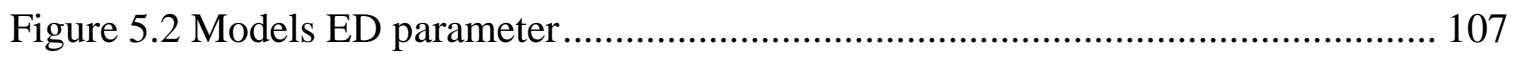

Figure 5.3 Effect of ED parameter on story drift ................................................. 110

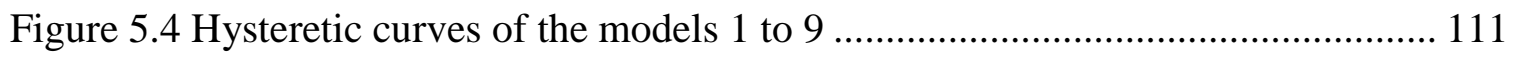

Figure 5.5 Points of interest within the link...................................................... 115

Figure 5.6 Effect of initial imperfections on PEEQ and hysteresis of models 1 and 9 .. 117

Figure 5.7 Effect of hw parameter on story drift ............................................... 119 
Figure 5.8 Hysteretic curves of the models 10 to 15

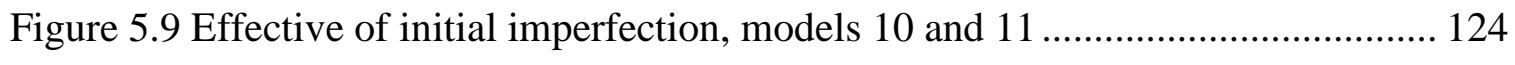

Figure 5.10 Hysteretic curves of the stiffeners thickness models.............................. 126

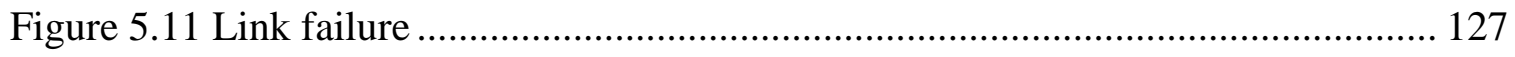

Figure 5.12 Two models to study the effect of opening edge on link performance ....... 129

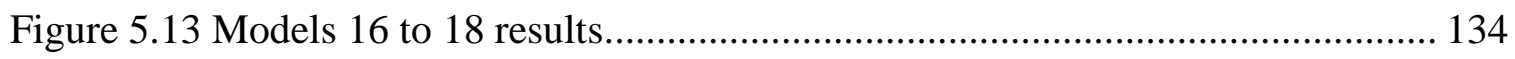

Figure 5.14 Models of common sections ….................................................... 138

Figure 5.15 von Mises stress distributions........................................................ 141

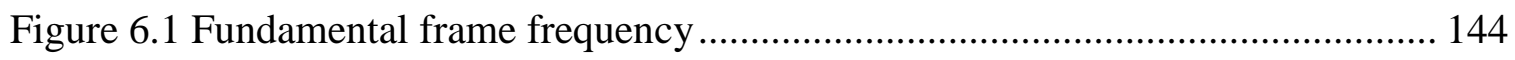




\section{Chapter 1: Introduction}

\subsection{General}

Eccentrically braced steel frames (EBF) are primarily designed and used for resisting lateral forces. EBF is described as a seismic resisting system compromised of two types of frames: concentrically braced steel frames (CBF), which have large lateral stiffness but low ductility, and moment resisting frames (MRF), which exhibit high ductility but a poor ability to support lateral loads. The bracing member in EBF gives it its stiffness, while the link gives it its ductility. EBF's were presented at the end of the 1970s as innovative steel frames designed according to the era's seismic design philosophy and performance criteria. Figure (1.1) shows the three different types of frames MRF, CBF and EBF.

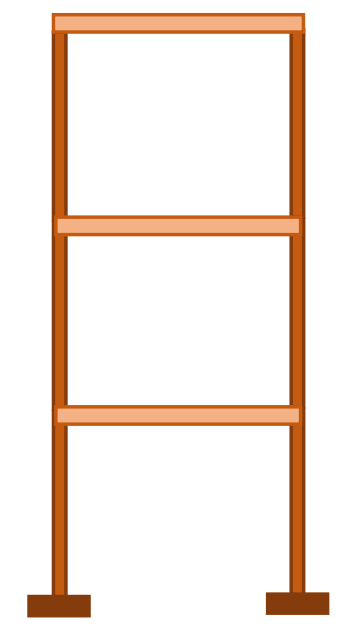

a) MRF

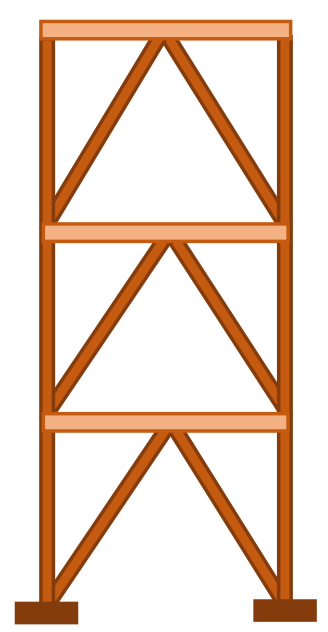

b) $\mathrm{CBF}$

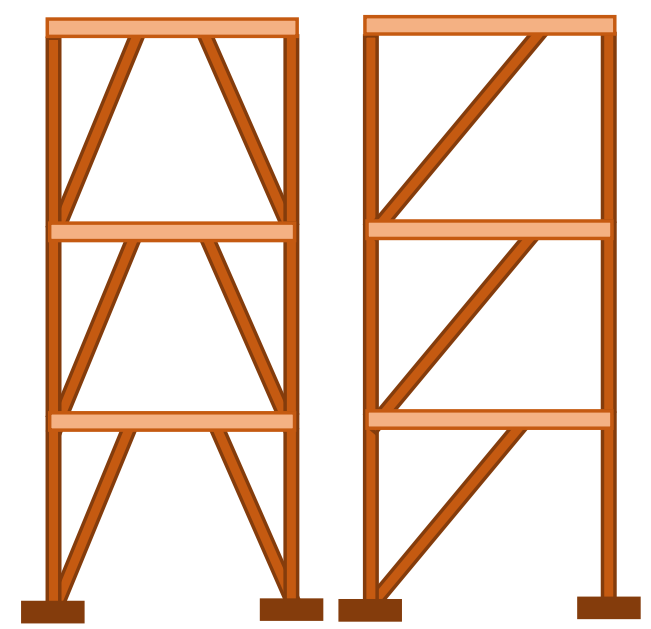

c) Examples of EBF

Figure 1.1 Three types of frames described above 
There are two main link configurations in EBF depending on the connections. The first one is called "K-Braced," where the link is eccentrically connected between two bracing members with the beam centerline. The second configuration is named D-Braced, where the link is adjusted to the column and the link is connected directly to it. In addition, there are also two types of EBF depending on the replaceability of the link: nonreplaceable links (conventional link) in which the link and the beam are in the same section and depth without need to change the link, and a replaceable link, wherein the link is a movable part and is bolted with the beam and/or column through thick plates. The concept of replaceable links in an EBF system appeared in the early 1980s as an easy way to remove the damaged links after a severe earthquake. Usually, the replaceable link has smaller depth than floor beams in order to overcome the oversized section problem, see Figure (1.2) for EBF configurations illustrated.

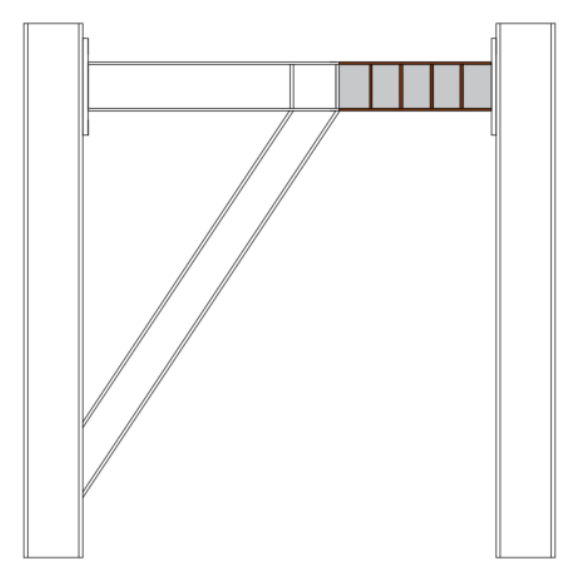

a) Conventional D-Braced EBF

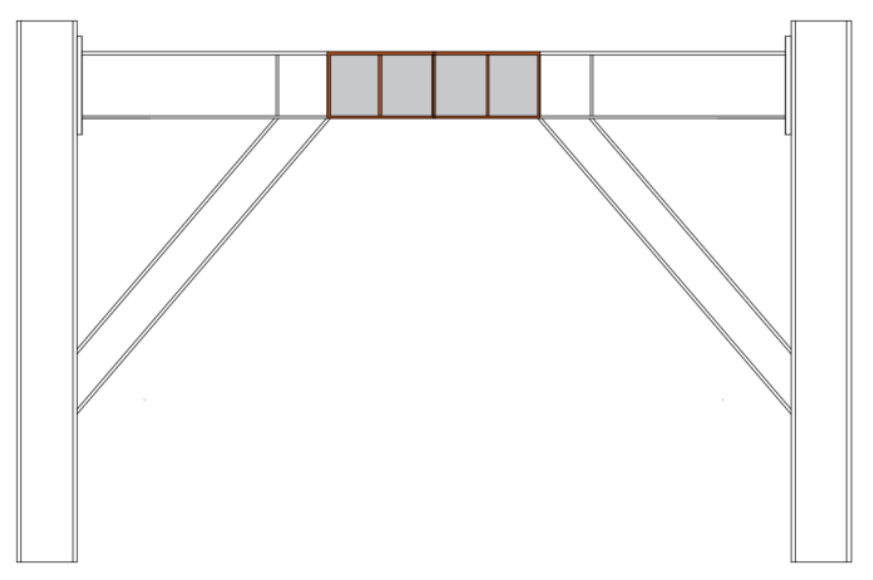

b) Conventional K-Braced EBF 


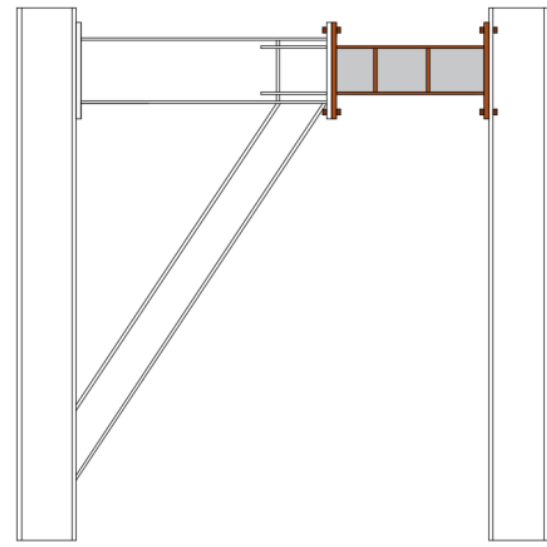

c) Replaceable D-Braced EBF

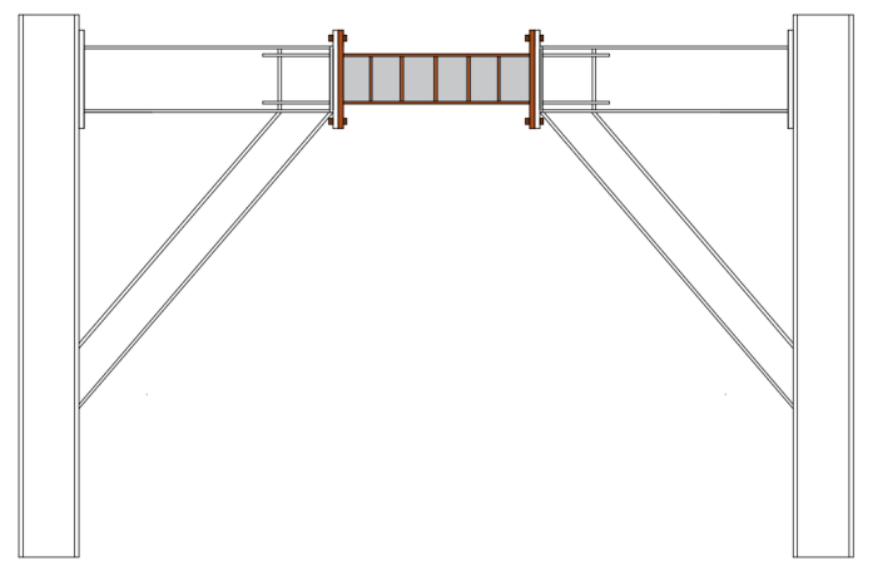

d) Replaceable K-Braced EBF

Figure 1.2 Some types of EBF

EBFs are assembled by many components such as columns, beams, connections, and braces. In most seismic design provisions, EBFs are designed to be part of a building's gravity resisting system and to resist the forces developed outside the link due to strain hardening. Therefore, following seismic design requirements leads to oversized link in conventional EBF, resulting in a need for other oversized components and foundations that increase the cost of the construction. Even though replaceable links can be a solution to address oversized link sections in EBF, the additional cost of detailing bolted links compared with conventional links may not be the best solution for noncritical buildings. Thus, the conventional EBF of a single continuous link and beam member is the most EBF configuration used (ANSI/AISC 341-16, 2016). Therefore, finding a way to modify the link in conventional EBF is still a valuable potential alternative for strength control. 


\subsection{Eccentrically Braced Frames Damages and Repairs}

\subsubsection{Link Damages}

A major earthquake can cause potential damage to the structure and the infrastructure. Control of damage in the structure can only be possible if the structures are designed according to a good seismic design procedure. Damages depend on the design criteria and types of structure. Cost plays a key role in determining the damage control design.

The design of structural steel buildings under seismic loading has been studied and improved upon for several decades. However, reducing building damages after an extreme earthquake is still a primary objective of researchers, not just to reduce the cost of repair, but also to resume an operational building. To reach these goals for the steel structural system, studies show that the structure must be ductile in order to achieve an inelastic deformation during the earthquake. Since the concept of eccentrically steelbraced frames was first introduced, many studies have focused ways to improve this type of frame which can protect the building from excessive damages by forming a fuse at the link location and then either by replacing or repairing the link.

EBF link damages are classified into four major types: web/flange yielding, web/flange buckling, slab concrete damages above the link, and web/flange fracture. Not all cases require replacement of the link, and the link should only be replaced in the case

of link fracture. Moreover, repair of the link may require simple cosmetic repair as in the case of the yielding of the web and the flange (Gulec, et. al 2011). 


\subsubsection{EBF Repair}

There are four ways to repair damaged EBF link which are connected with damage states as shown below (Gulec, et. al 2011);

Cosmetic repair: In this repair, it is unnecessary to regain strength and stiffness of the link and the repair does not affect on the performance of the link.

Concrete damages: due to vertical deflection of the link, the concrete above the link may be damaged. The repair process is done by either replacing the concrete or by injecting epoxy into cracks.

Heat straightening: heating can treat web buckling and flange buckling in minor cases.

Link replacement: removal and replacement are required when fractures appear in the link web or flanges.

\subsection{Research Background}

EBFs in their current configurations were previously suggested as an innovative solution for a seismic resisting system that can dissipate earthquake energy through a structural fuse and can prevent buckling of the brace through the inelastic deformation that can occur in the ductile link during a severe earthquake (Roeder and Popov, 1977). The web stiffeners are used in the link to give it the necessary stability and to distribute shear stress in the web of the link. They also work to avoid any possibility of local yielding or web crippling that may occur. 
The specimens used in early EBF experimental work during the 1970s and 1980s were built from steel materials using A36 grade steel. Results from those studies showed that the links were stable and can dissipate earthquake energy through inelastic shear deformation (Hjelmstad and Popov, 1983). More studies on the effect of material properties were also experimentally tested later (Okazaki et al., 2005; Chao et al., 2006; Dusicka et al., 2009). Research on very short links showed that a very high overstrength factor, about $\Omega=5$, can be achieved with high rotation when a low-yield stress steel is used in very short links and without using web stiffeners (Dusicka et al., 2009). In contrast, studies on the behavior of the links in EBF with steel containing a higher yielding point such as A709 and A992 have demonstrated that initial fractures start at the end of the stiffeners - to - web weld before observing any web buckling (Okazaki et al., 2005). Attempting to modify the web stiffener details in the link showed that stiffener arrangement has a limited effect on the link fractures and can only delay the web fractures and enhance the link rotation capacity, but only through welding to the flanges (Okazaki et al., 2007). More research regarding the stiffener configurations and the influence of the material properties of the $\mathrm{k}$-area on the link web fracture was recommended by Okazaki et al. (2007). Numerical study on the effect of material properties of the k-area has shown that a k-area with low-yield material can reduce the potential of web ductile fracture at the end of the stiffeners because material with a lowyielding point allows yielding to penetrate deeper into the $\mathrm{k}$-area, resulting in an increase within the yielding region at the web gap and a reduction in the local ductile fracture demand (Chao et al., 2006). Depending on the numerical results, Chao et al. (2006) 
suggested use of a single horizontal stiffener instead of employing many vertical stiffeners to avoid welding near the k-area.

The extended end plate used in link-to-column connections was suggested by Ramadan and Ghobarah (1995) as shop-welded link-to-end plate and the end plate is bolted to the column flange. The performance results of the bolted extended end plate were similar to that of fully welded connections. Later, experimental study by Dusicka and Lewis (2010) on a new method of using parallel stiffeners near the end plate connections in the replaceable link in linked column frame, LCF, showed that using end stiffeners with bolted extended end plate can improve the link performance and reduce the plastic demand and move the plastic strain away from the link-to-end plate weld. Figure (1.3) shows the test setup of the bolted end plate in replaceable links.

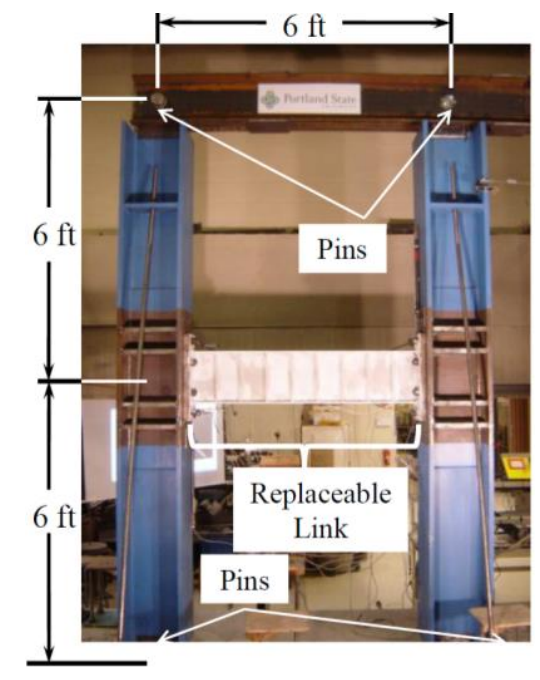

Figure 1.3 Test setup for bolted end plate replaceable links (Dusicka and Lewis, 2010)

Because the link-to-column connection is an asymmetrical link, the elastic moment at the shear link end is much larger than the other end link moment (Kasai and 
Popov, 1986). In the link-to-column configuration, high axial stiffness of the column and less flexural stiffness of the link can cause fractures of the link flange at low rotation. Fractures at the link flange to endplate connection have been observed before the link reaches the target rotation (Okazaki et al., 2006). Therefore, link-to-column connection in EBF remains a field in need of ongoing research, as indicated by the AISC in 2016 due to the poor performance of the links.

Moreover, more data on the behavior of EBF were collected during the 2010 and 2011 large earthquakes which hit Christchurch in New Zealand, inflicting massive damages. This event represents the first recording of actual behavior of EBF and served as a very real test of it. Reports and studies published subsequently have shown relatively better behavior of steel structures than other types of structural systems as EBF performed satisfactorily in the studied buildings (Clifton et al., 2011). However, unpredictable fractures appeared in some links which were designed to attain inelastic deformation. The inelastic deformation of the link in EBF dissipates the seismic energy. The amount of dissipation depends on the ductility of the link and its ability to deform largely without fracturing. Degree of damages might be varied, and the repair cost plays significant role in the design principles (Kanvinde et al., 2014). The damages of conventional links can be difficult and expensive to repair since the link is not separated from the collector and serves as part of floor beam.

Reducing the web area of the shear links of EBFs has been investigated in previous studies. A numerical study was conducted on the behavior of the link in EBF with reduction of the web area by drilling holes. The aim of forming holes in the web link 
is to reduce link-flange connection stresses and improve the connection rotation capacity. Finite element analysis revealed that forming holes in the web link can achieve these goals, however, it increases the stresses triaxiality, as well as the plastic strain in the link web (Prinz and Richards, 2009). Perforated replaceable cast steel links under cyclic loading were tested experimentally and compared with specimens without circular perforations (Tong, et al., 2018). Results have shown that links without web opening have more ductility than links with web perforation and can achieve a rotation angle up to 0.17 rad. Initial cracks of specimen with web opening, labeled L-C-2, were noticed at low link rotation of $0.03 \mathrm{rad}$. The cracks formed at the hole center of the middle web panel and increased with increasing of the link rotation. In opposite to the reducing the web area, reducing a flange area in the EBF link-to-column connection is an optional solution. Plastic strain at the end of the links can drop dramatically with removal of the flange area, thus achieving better link ductility (Berman et al. 2009).

Reduction of the flange area is not the only option to improve the link performance, the yielding mechanism can also play a significant role in the behavior of the links in EBF's. Among the four yield mechanism types used in Tan and Christopoulos (2016), the simultaneous flexural yielding link concept behaved the best. This type is based on the concept that the cross-sectional area of the shear link is proportional to the shear force applied over that length and the deformation of the segment contributes to overall link deformation. A varying width-rectangular hollow section was suggested for use in the link since it prevented failure modes, such as web buckling, weld metal fracturing, and lateral torsional buckling. The links can achieve up 
to double the rotation capacity specified in the AISC seismic code. Theoretically, using replaceable build-up links with wide, thick flanges and thin webs that are bolted with the controller beam through the flanges and web splicing can achieve the seismic design requirement. In this model, the link depth is relatively less than the beam depth (Ashikov and Clifton 2016).

The concept of using Tuned-Eccentrically Braced Frame, T-EBF, was conceptually suggested by (Volynkin, 2016) during his doctoral study. Numerical models were pushed over to investigate the stresses on T-EBF and some design ideas were suggested. However, his suggestion did not involve any experimental work or cyclic analysis. More control over link strength can be achieve by using T-EB and more wide flange sections can be utilized as a link in T-EBF. In this study, conventional continuous beams with reduced web section of the shear link were proposed and evaluated experimentally in order to control the link strength. The idea of reducing web area serves to ensure that any plastic deformation can only occur in the link.

For the design, Overstrength factor $(\Omega)$ is one of the primary parameters necessary to determine. It is used to calculate the maximum forces developed in the link and is defined as the ratio between the ultimate shear strength and the plastic shear strength. Depending on early experimental works undertaken at the University of California, $(\Omega)$ ratio was suggested to the value of 1.5 (Hjelmstad and Popov 1983; Malley and Popov 1983). However, AISC seismic provision reduces $(\Omega)$ factor to 1.25 for design of the diagonal strength. Previous tests on large scale build-up sections used in the bridge demonstrated the overstrength factor increases up to 2 (Dusicka et al. 2002). In 
this literature, to the present researcher's knowledge, there is a lack of experimental investigation regarding the overstrength factor of the reduced section area in the links. The other goal of this study is to find the ultimate strength of the links with section reduction.

\subsubsection{Failure Observed from Researchers}

Fractures in the link web are observed when high-yield stress steel is used as shown in Figure (1.4). The fracture begins at the fillet weld between the web and the top or bottom of the stiffener then grows along the web, resulting in strength degradation. This type of failure was not observed during previous studies of 1970's and 1980's. The seismic design procedure of available EBFs today depends primarily on these earlier studies. Therefore, many researchers have addressed this issue and suggested that the main reason for the appearance of ductile web fractures is the result of the types of materials used today, which are different from the materials used previously when these studies were first undertaken (Chao et al. 2006; Okazaki et al. 2007; Dusicka et al. 2009). EBF has different types of shapes, and among all types of EBF configurations, the link to - column is still not recommended for use until further studies have been undertaken, as indicated by the AISC in 2016 due to poor performance of the links. 


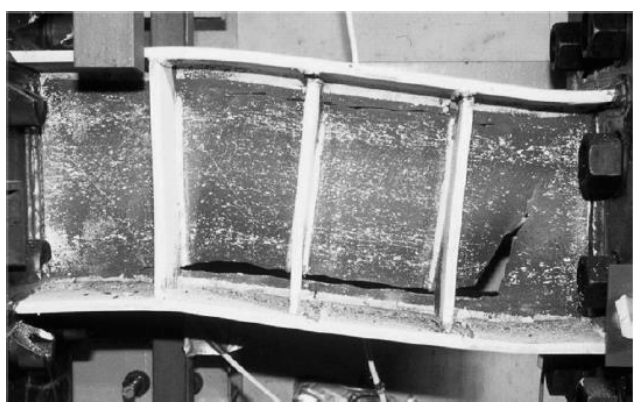

a) Fracture at $\gamma=0.06 \mathrm{rad}$ (Okazaki et al 2007)

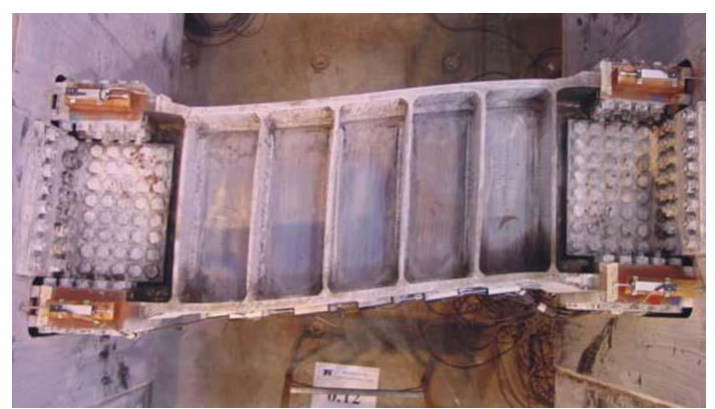

b) Initial cracks at $\gamma=0.07 \mathrm{rad}$ (Dusicka, 2009)

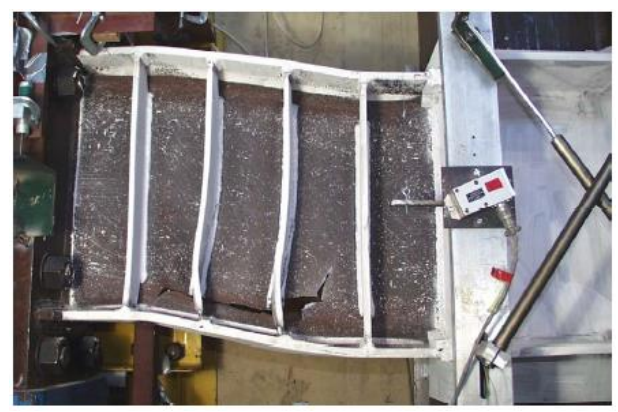

c) Fracture at the link-to-column connection at $\gamma=0.071 \mathrm{rad}$ (Okazaki, 2004)

Figure 1.4 Fractures observed in some EBF links during tests

\subsubsection{Failure Observed from Real Practice}

After a series of earthquakes hit Christchurch in New Zealand in 2010/2011,

EBFs were put into real practice. Reports show many EBF links were suffering extensive damage as shown in Figure (1.5a, b and c) (Clifton, et al. 2011). Even in new buildings, such as a 22-story building built in 2009, some links in the EBF suffered severe damages, as shown in Figure (1.5d).

These high-risk concerns suggest a need to find a way to improve the design of the link in EBF. EBFs also have been widely used to retrofit buildings. 


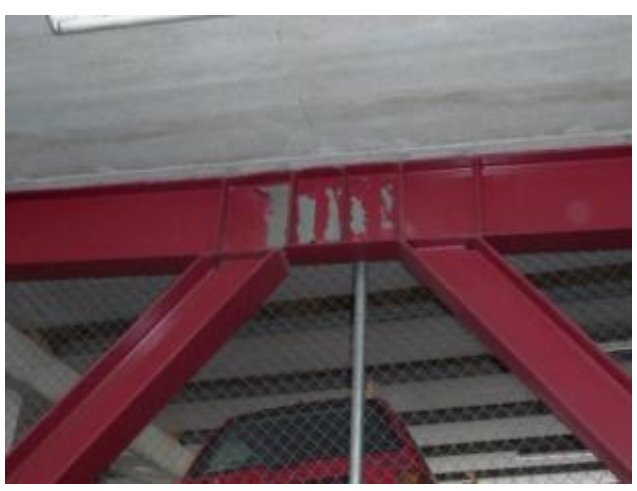

(a) Yielding of EBF link

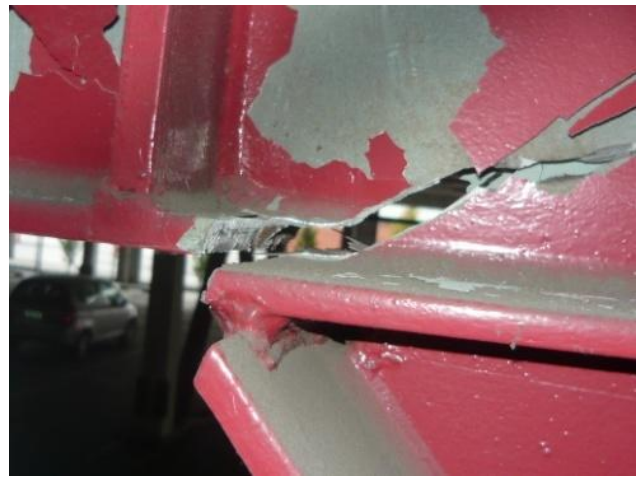

c) EBF link fractures in a garage

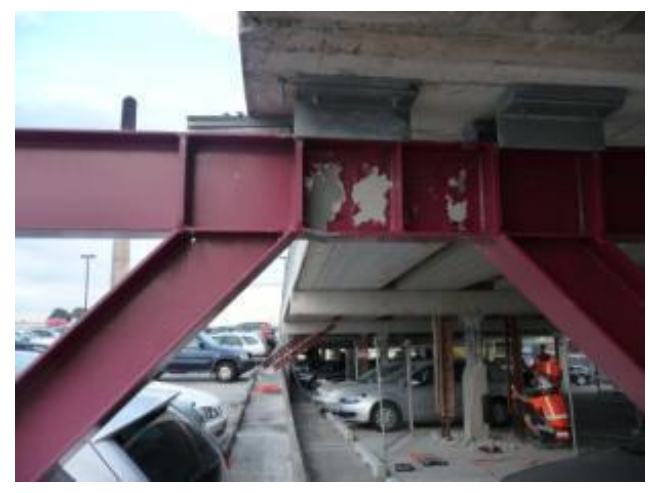

b) Flange local buckling

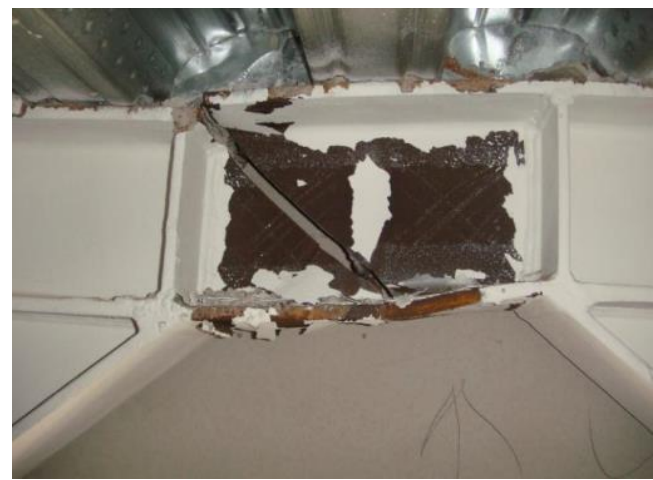

d) EBF link fractures in a building

Figure 1.5 Various types of earthquake damages appeared in EBF (Clifton, et al. 2011)

\subsection{Brace-to-Link Connections}

The important details of the brace-to-link connection can play a significant role in the stability of EBFs. Therefore, in the current AISC seismic provisions (ANSI/AISC $341-16,2016)$, the required strength of the diagonal brace connection should be the same strength as the diagonal brace. The connection is designed as a fully-restrained, momentresisting connection. Brace-to-link connections were studied and tested in previous research during the 1980's. A six-story building with EBF's was tested using tube braces. 
Stiffened gusset plates were used to connect the tube braces with the links. A simulated earthquake with a half-ground acceleration was applied to the building and the test extended beyond the designed level to detect the failure mechanism. The test demonstrated that the yield was followed by buckling of the guest plate causing the end of the brace to move out of the plane, which in turn resulted in lateral torsional buckling in the girder close to the shear link. Therefore, the brace no longer had the ability to transfer forces into the link, limiting its strength and energy dissipation capacity (Foutch et al., 1986).

The three most common types of brace-to-link connections used today were proposed and tested in fourteen $2 / 3$ scale subassemblage models subjected to cyclic loads in order to investigate the stability of the connections during inelastic deformation of the links (Popov et al., 1989). These typical connections, shown in Figure (1.6a, b, and c), include a direct welded connection for $\mathrm{W}$-section brace, a direct welded connection for the tube section brace, and a stiffened gusset plate for the tube section brace. All these types performed well during testing and they have since been adapted and recommended by the AISC seismic design provision (ANSI/AISC 341-16, 2016).

In the brace-to-link connections, the vertical stiffeners are welded to the web on both sides to prevent web crippling or local yielding and to reduce stress concentrations. Several studies have shown that misalignment of the beam-brace flange with the link stiffeners point, such as the link shown in Figure (1.5c), can cause premature fracturing outside of the link due to stress concentration (Imani et al., 2015; Kanvinde et al., 2014). 
Therefore, the AISC provision warns designers that even a small offset can affect performance of the link.

The aim of this research is to investigate the performance and behavior of TunedEccentrically Braced Frame (T-EBF) within two different brace- to-link connection configuration and estimate the efficiency of bolted brace-to-beam connections.

The typical brace to link connections shown in Figure (1.6a, b,and c) are the common types used in practice. The direct welded tube and $\mathrm{W}$ section connections are used in different EBF configurations when a large amount of movement is expected in the brace, as in the case of long links. The stiffened gusset plate connection is one of the most popular brace-to-link connection types and is often used. The brace can be either be welded or bolted to the plate and the plate also can be welded or bolted to the beam. The gusset plate is designed to be stable for both tension and compression loading under the cyclic reverse. The failure of the gusset connection under tension might be result in either bolt or weld fracture, or block shear failure of the gusset plate, while the buckling gusset plate is the type of failure found in compression loading. In case of the initial imperfection or plate buckling, the lateral torsional buckling is also a possible mode of failure of the beam. The beam outside of the link can also be damaged through repeated inelastic deformation during seismic design level (Mansour et al., 2011). In this research, welded and bolted brace-to-link connection of T-EBF was experimentally tested. The proposed bolted connection is expected to contribute to finding a solution to the problems related to beam fracture or damage outside the link since this type of connection is more practical in that engineers can disconnect the parts in case of replacement and, at the 
same time, sustain the undamaged bracing member. In addition, the bolted brace-to-link connection can add stiffness to the beam flange due to use of thick plates and reduce stress concentration because of the misallocation of the vertical stiffness and lessen the need for field welding.

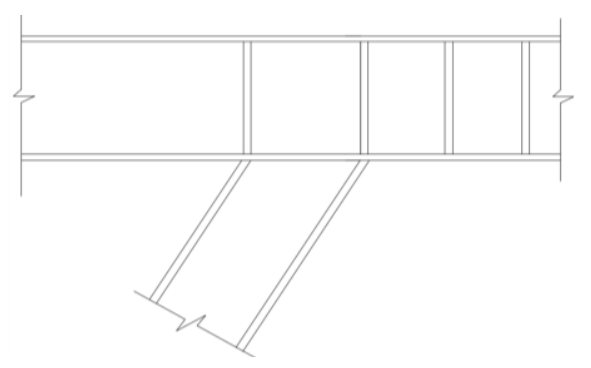

a) Direct Welded W-Section

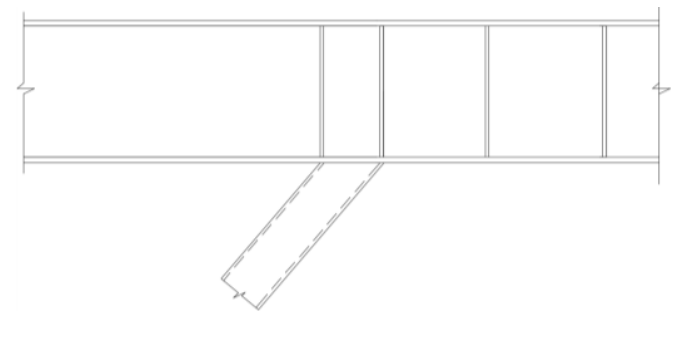

b) Direct Welded Tube

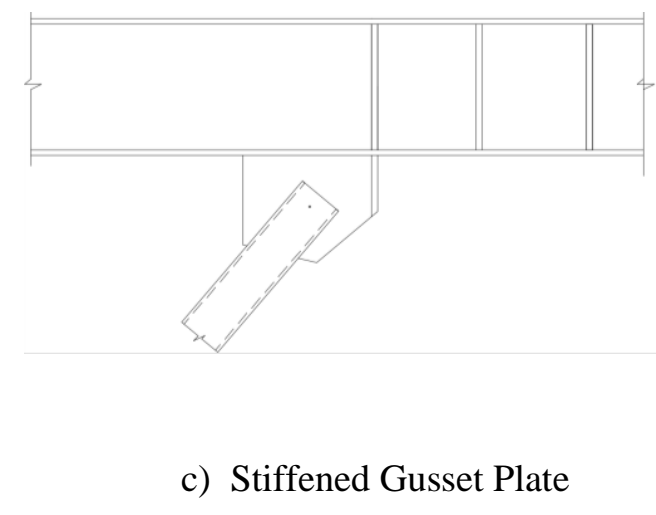

Figure 1.6 Brace-to-Link connection

\subsection{Motivation of the Study}

- The poor performance of the link to column connection, which leads to the AISC seismic provision (ANSI/AISC 341-16, 2016) suggests not using this type of EBF until more studies are coming. 
- Find a way to control the link strength to overcome the oversized design problem (braces, columns, floor slabs, connections and foundations) in conventional EBF which is still the preferable type due to economic issues and construction time.

- Repairing the damaged conventional EBF is difficult as the entire beam and link might require replacement. This fact has motivated researchers to find ways to improve the performance of link to column connection.

- Welded a bracing member to a collector beam can require greater time and cost to repair after a major earthquake than the suggested method of bolting the bracing member with the collector beam.

\subsection{Objectives}

The study objectives can be summarized as follows;

- Develop a Tuned-Eccentrically Braced Frame, T-EBF in a conventional, nonreplaceable link-to-column connection, and experimentally evaluate the performance of the suggested opening configurations of the link. The aim of reducing the web section in the link is to limit the capacity strength of the link so that it can work as a structural fuse capable of successfully dissipating earthquake energy through inelastic deformation and reduce the transferred forces and moments to the column and beam outside the link.

- To find experimentally the overstrength factor for reduced web section.

- Propose a new bolted diagonal brace connection in T-EBF that can be easily disconnected for the repair and replacement of damaged components without needing to replace the bracing member after a design-level seismic event. 
Reducing the stress concentration at the flange brace and stiffener edge connection is also a preferable feature of this type of connection.

- Develop a nonlinear analytical model to study the opening configuration of the proposed tuned link and the effectiveness of the main parametric on the general performance of the frame and local strains developed during the cyclic loading.

- Recommend design guidelines for using T-EBF in seismic design of the link-tocolumn connections.

\subsection{Dissertation Organization}

The dissertation will be divided into seven chapters as follows:

- Chapter 1 Introduces the topic and a literature review on the topic and offers the necessary background of the research will discuss the motivations and objectives of the study.

- Chapter 2 Describes the experimental program, test setup, the specimens, and ways to measure the variables required in this study, such as an instrumentations map and methods to calculate deformation and forces.

- Chapter 3 Describes the test results of the four specimens including the two brace to link connection types.

- Chapter 4 Describes the analytical part of this study, including the model definition and the convergence study. Validation of the model versus the experimental tests is also will be checked in this chapter in terms of the global behavior. 
- Chapter 5 Illustrates the parametric study of the link in the tuned eccentrically braced frame, T-EBF, and discusses the effect of the parametric values on the link behavior.

- Chapter 6 Gives design guidelines for T-EBF

- Chapter 7 Includes both conclusions and recommendations.

- Appendix: Design Calculation, research drawings, coupon test report. 


\section{Chapter 2: Experimental Specimens and Test Setup}

\subsection{Introduction}

Four full-scale specimens were used here to apply the concept of Tuned Eccentrically Braced Frame, or T-EBF. T-EBF was implemented in this research on Dbraced EBF, where the link is connected to the column. The specimens were designed and fabricated in accordance with the AISC seismic provision (ANSI/AISC 341-16, 2016) and the test procedure was based on the prequalification and cyclic qualification of link to column connections in an eccentrically braced frame, as described in the provision. All the experiments were done in the iSTAR lab at PSU. The major components of the test system were main test floor, reaction frame, and T-EBF. The test employed two experiment phases to study the stability of the tuned link in EBF, and to ensure that they could achieve the seismic design prediction, in which the link dissipates earthquake energy while the other frame parts remain within elastic range. The first phase of T-EBF contained two specimens of different dimensions, W18x76 and W16x67, in which the brace member was welded to the beam. The second phase also consisted of two specimens: W18x76 and W16x67. However, in phase two, the brace was bolted to the beam. The tests were in plane only. This chapter illustrates the experimental program in detail by providing an overview of the test setup and testing procedures, including T-EBF specimens, instrumentation plans, and cyclic loading protocols.

\subsection{EBF Design Requirements}

In the conceptual design of EBF, yielding, inelastic deformation, and damages occur within the link. Therefore, the link should be designed as the weakest part of the 
frame. In addition, the strength of the other components, such as the brace, connections, columns, and beams, is estimated depending on the forces developed in the link due to strain hardening. The success of the link relies on its ductile behavior. To achieve the target ductility, the link length, overstrength factor, and rotation capacity should be estimated and the link should be designed to meet the rotation demand. Therefore, the link design must follow seismic design codes. Some of the design guidelines suggested by Popov adapted by AISC seismic provision (ANSI/AISC 341-16, 2016) are presented below.

\subsubsection{Link Length (e)}

The types of the link are classified into three categories depending on length (e): shear link, intermediate link, and flexural link. The large variety of link lengths is due to the wide range of shear-flexural interaction resulting from the strain hardening of the links. Based on the previous experimental works, the link types can be limited as shown in the expressions below.

$$
\begin{array}{ll}
e \leq 1.6 \frac{M_{p}}{V_{p}} & \text { shear link } \\
e \geq 2.6 \frac{M_{p}}{V_{p}} & \text { flextural link } \\
1.6 \frac{M_{p}}{V_{p}} \leq e \geq 2.6 \frac{M_{p}}{V_{p}} & \text { intermediate link }
\end{array}
$$

The shear link has a high capacity for energy dissipation and ductility and is recommended for use when the link is attached to the column (Kasai, K., \& Popov, E. P., 1986). In contrast, the long link should not be used in column connection due to a link 
flange fracture that appeared before the link achieved the required rotation (Engelhardt, M. D., \& Popov, E. P., 1992).

\subsubsection{Link Rotation}

The phrase 'link rotation' is used to describe the link ductility in EBF. The total link rotation is denoted as $\gamma_{\text {total }}$. The main goal of EBF is to achieve a link rotation greater than the rotation demand of the seismic design. Link type and link rotation are related as shown in Figure (2.1). Shear links should be designed to reach a rotation of 0.08 radian, while the rotation demand of the flexural link is 0.02 rad. Interpolation should be used to estimate the link rotation demand for intermediate links.

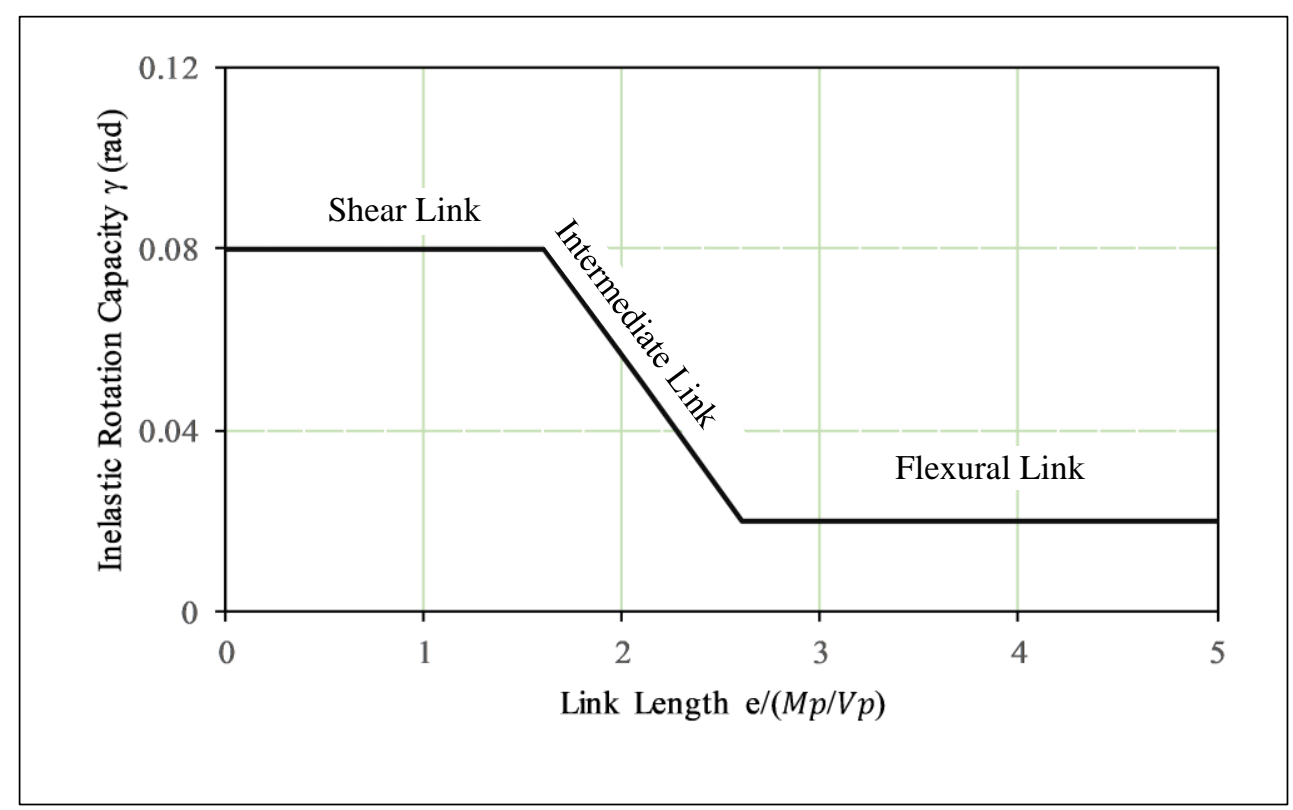

Figure 2.1 Link types related to the link rotation and length

The rigid plastic mechanism illustrated in Figure (2.2) illustrates the relationship between the plastic story drift angle and the link plastic rotation (Kasai, K., \& Popov, E. 
P., 1986). The expression below shows that increasing the ratio of (L/e) for a given plastic story drift $\left(\theta_{\mathrm{P}}\right)$ increases the link rotation angle $\left(\gamma_{\text {total }} \approx \gamma_{P}\right)$. Figure $(2.2)$ also shows the energy dissipation mechanism for type D-EBF.

$\gamma_{P}=\frac{L}{e} \theta_{P}$

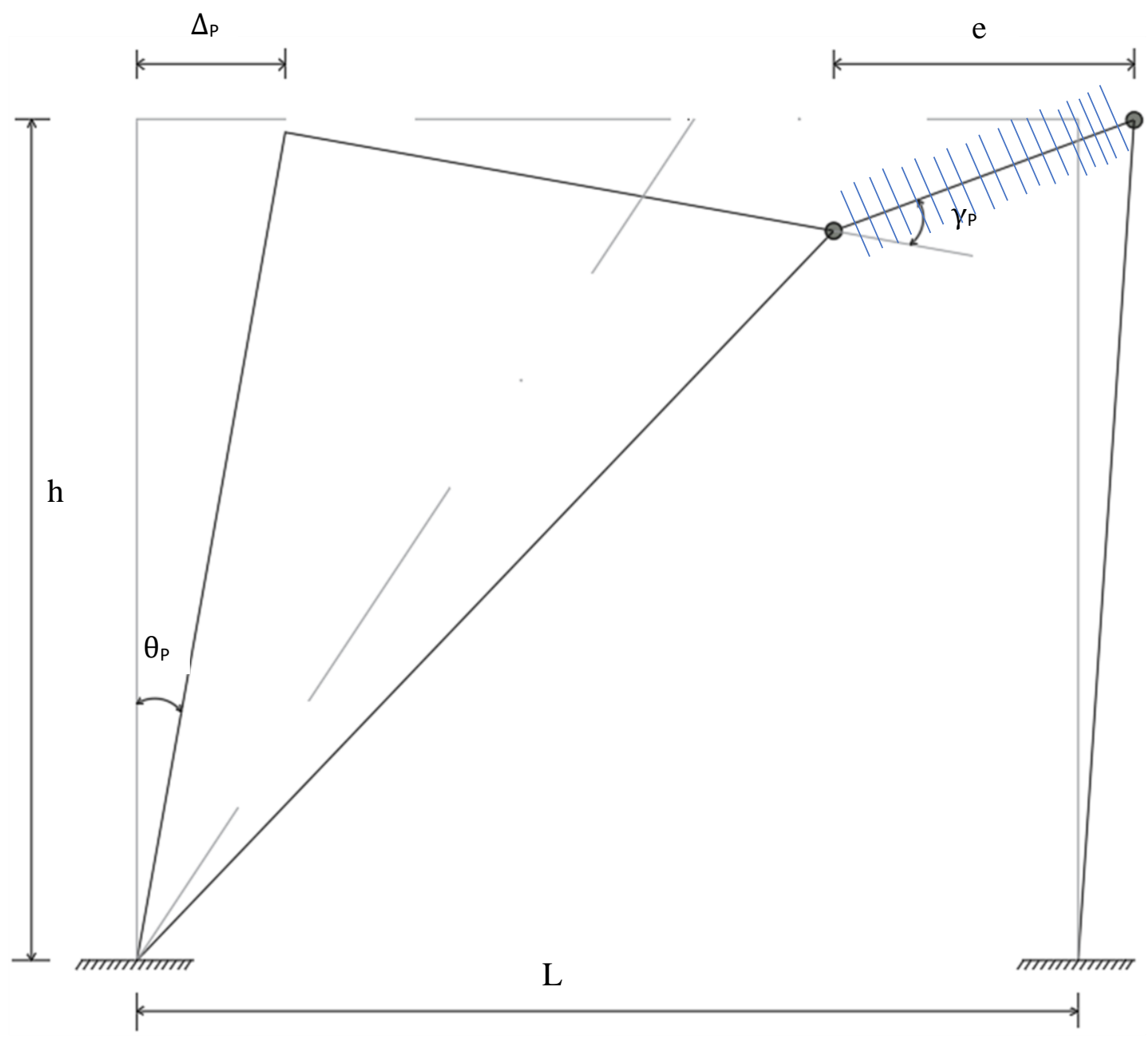

Figure 2.2 Energy dissipation mechanisms 


\subsubsection{Forces in Links and End Moments}

Because the link to column connection is asymmetrical, the elastic moment at the shear link end is much larger than the other end link moment (Kasai and Popov, 1986). However, as the link shear force rises, and the link rotation increases, and the ends moments come to be equal. Therefore, for the purpose of simplification, researchers usually neglect the differences between them. Moreover, high axial stiffness of the column and less flexural stiffness of the link is another feature of the link-to-column configuration. In general, the link carries high end moments, high shear force, and low axial loads. From the free body diagram (FBD) of an isolated link, subjected to coupled shear forces (V) and end moments (M) shown in Figure (2.3), the length of the link is related to these forces by the below expression:

$e=\frac{2 M_{P}}{V_{P}}$

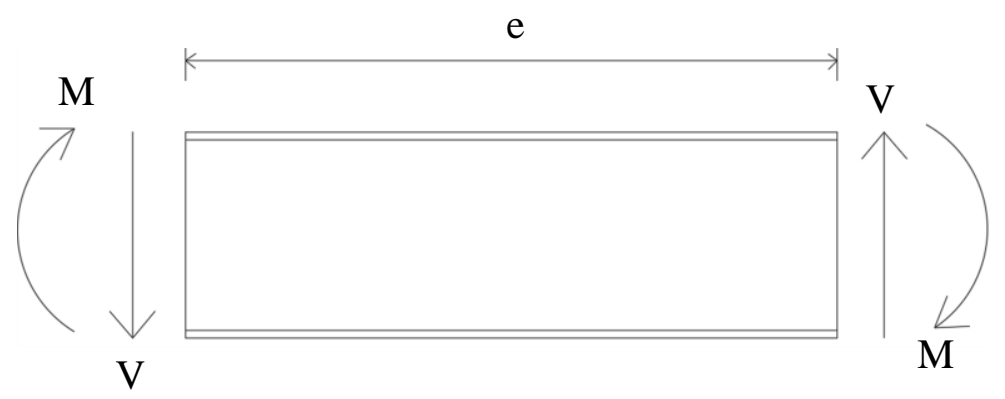

Figure 2.3 FBD of a link

Here, $V_{P}$ and $M_{P}$ are the fully plastic shear and moment for link of elastic perfectly plastic. 


\subsubsection{Bracing Arrangement}

It is important to choose the bracing configuration in appropriately to ensure stable inelastic behavior within the link. The bracing member should be able to resist very high axial loads in both tension and compression. The brace to link connection must be designed for the same bracing member forces. In practical design, the link strength must be at least 1.5 times the nominal link strength in order to prevent the brace buckling. The angle between the link and the diagonal brace should be large enough to reach the required design capacity.

\subsection{Specimen Design}

Specimen design calculations are included in the appendix A. The frame columns were designed and left from a previous test (Volynkin, 2016). The frame member sizes were adjusted to the actuator load capacity, which is 220 kips. The link strength, shear force, and deformation calculation are summarized below.

\subsubsection{Strength Calculation}

The strength of the EBF depends mainly on the strength and ductility of the link. The EBF components were designed following the development forces that resulted from fully yielded link and strain hardening. As mentioned before, the moment capacity at the shear link end to column connection may not be the same as the other link end. The strength capacity of the perforated link section, shown in Figure (2.4), is less than the strength of the unreduced link section. The plastic moment strength of the unperforated 
section depends on the yield stress $\left(\mathrm{F}_{\mathrm{y}}\right)$ of the material and on plastic modulus of the section (Z), as shown in Equation 2.6.

$M_{p}=F_{y} Z$

When the opening is located at the center of the section, the plastic modulus of the perforated section can be estimated by eliminating the contribution of the opening from Equation 2.6, as shown in Equation 2.7, where; $h_{o}$ is depth of the opening, $\mathrm{e}_{\mathrm{o}}$ is the eccentricity of the opening,

$M_{p}=F_{y}\left(Z-\frac{h_{o}^{2} t_{w}}{4}\right)$

When the center of the opening is offset from the center of the section $\left(e_{o}\right)$, another term is added to Equation 2.7, as shown in Equation 2.8 (Darwin, 1990).

$M_{p}=F_{y}\left(Z-\frac{h_{o}^{2} t_{w}}{4}-h_{o} e_{o} t_{w}\right)$

Since the plastic neutral axis is always located inside the horizontal stiffener, and to simplify the plastic moment equation, the effect of the horizontal stiffeners is taken to the center of the horizontal stiffener (equation 2.9), resulting in a more conservative plastic moment capacity. The terms $t_{h s}$ and $b_{f}$ are the stiffener thickness and flange width respectively.

$M_{p}=F_{y}\left(Z-\frac{h_{o}^{2} t_{w}}{4}-h_{o} e_{o} t_{w}+\frac{\left(t_{h s}\right)^{2}\left(b_{f}-t_{w}\right)}{4}\right)$ 
To evaluate the plastic shear capacity of perforated section, the remaining web area is calculated, and the contribution of the flanges is omitted, as shown in Equation 2.10.

$V_{p}=0.6 F_{y} A_{l w}, \quad A_{l w}=\left(d-h_{o}-2 t_{f}\right) t_{w}$

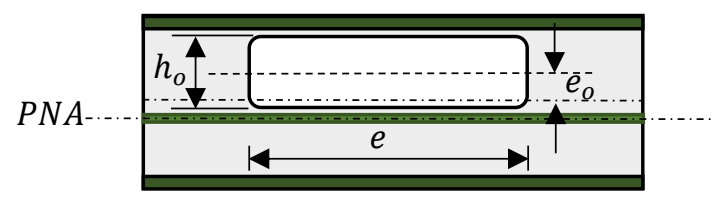

(a) T-EBF opening configuration

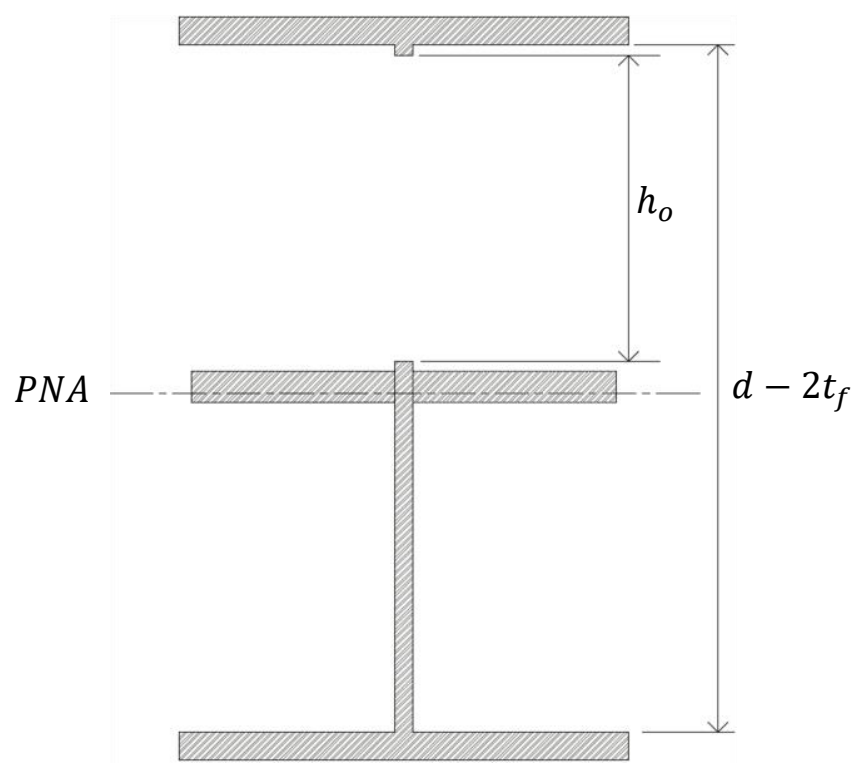

(b) T-EBF opening cross section

Figure 2.4 Perforated beam with web opening 


\subsubsection{Shear Calculation}

Link shear force $\mathrm{V}$ was calculated equal to the force reaction at the pinnedcolumn base plate, as shown in Figure (2.5). From the static equilibrium, the shear force is found as the equation below (2.11).

$V=\frac{P_{a c t . H}}{L}$

In the above equation, $P_{\text {act. }}$ represents the applied lateral load, $\mathrm{L}$ is the horizontal distance between the columns center to center, and $\mathrm{H}$ represents the vertical distance between the actuator and the center of the pin-supported column base.

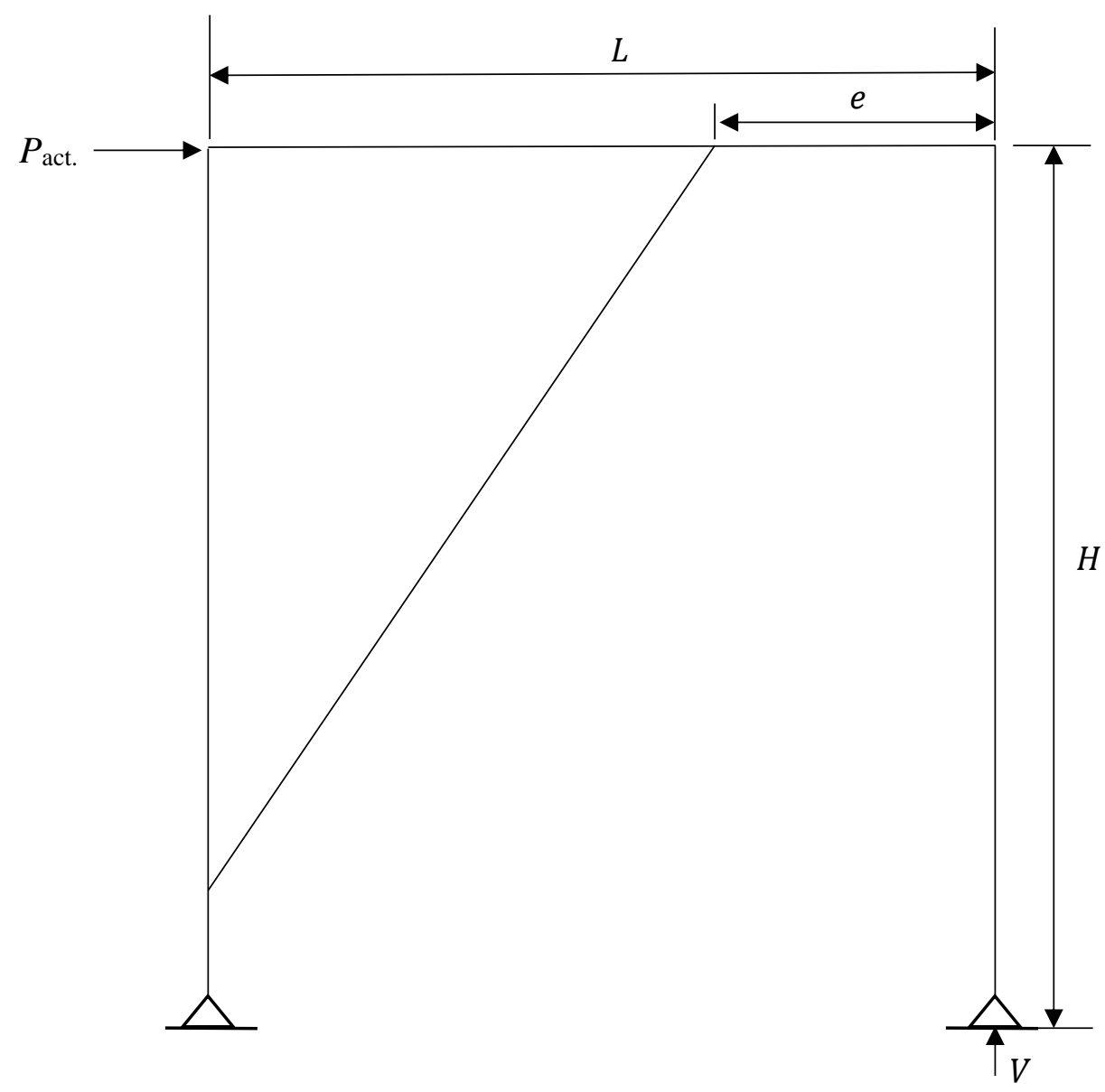

Figure 2.5 Link shear force in D-Braced EBF 


\subsubsection{Deformation Calculation}

The effective link rotation and the panel rotations were calculated through the linear variable differential transformers placed diagonally on the specimens. The rotation of the link panels was calculated depending on the diagonal deformation, as expressed in the following relation:

$\gamma=\frac{1}{2} \frac{(\delta 1-\delta 2) \sqrt{a^{2}+b^{2}}}{a b}$

$\delta 1$, and $\delta 2$, shown in Figure (2.6), were the deformations recorded during the test. The parameters a and $\mathrm{b}$ represent the vertical and horizontal distance between LVDTs of the deformable panel, respectively.

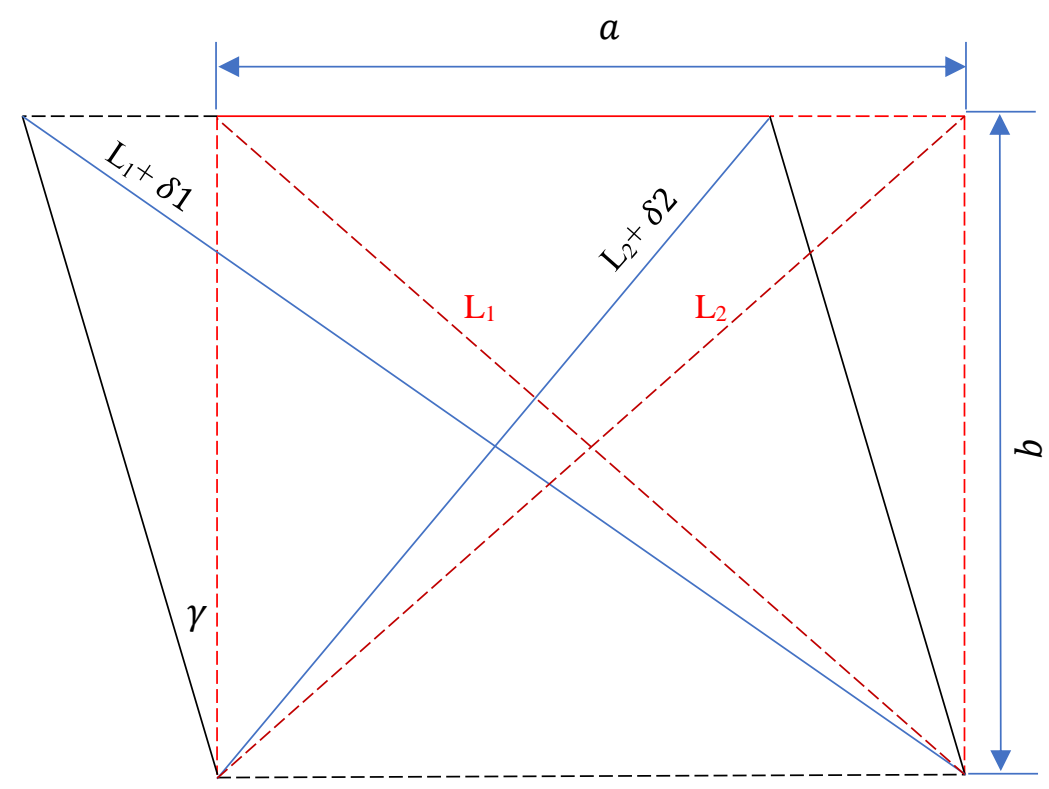

Figure 2.6 Geometry of shear deformation 
The effective panel is the one located under the web opening in the middle of the link, named BCFG in Figure (2.7). The panel rotation can be estimated using equation (2.13). A total of twelve LVDTs were used for this purpose in the six link panels, as presented in Figure (2.7).

Rotation_BCFG $=\frac{1}{2} \frac{(\text { Change length_BG-Change length_CF }) \sqrt{B C^{2}+B F^{2}}}{(B C)(B F)}$

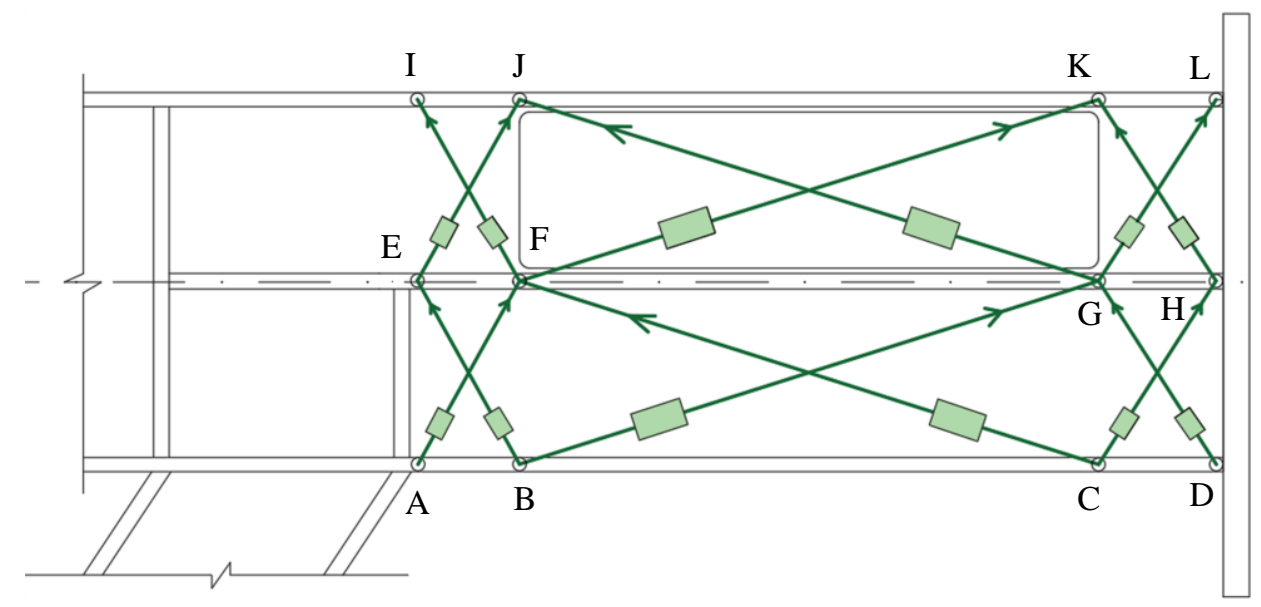

Figure 2.7 Link with six panels, using LVDTs

\subsection{Loading Protocol}

Quasi-static cyclic lateral load tests were carried out on four full scale specimens to evaluate their seismic performance. The loading protocol used in this study was adapted by AISC Seismic Provision (ANSI/AISC 341-16, 2016). The shear link achieved 0.08 rad before any degradation or loss of its strength. Figure (2.8) represents the required number of cycles against each increment of rotation. 


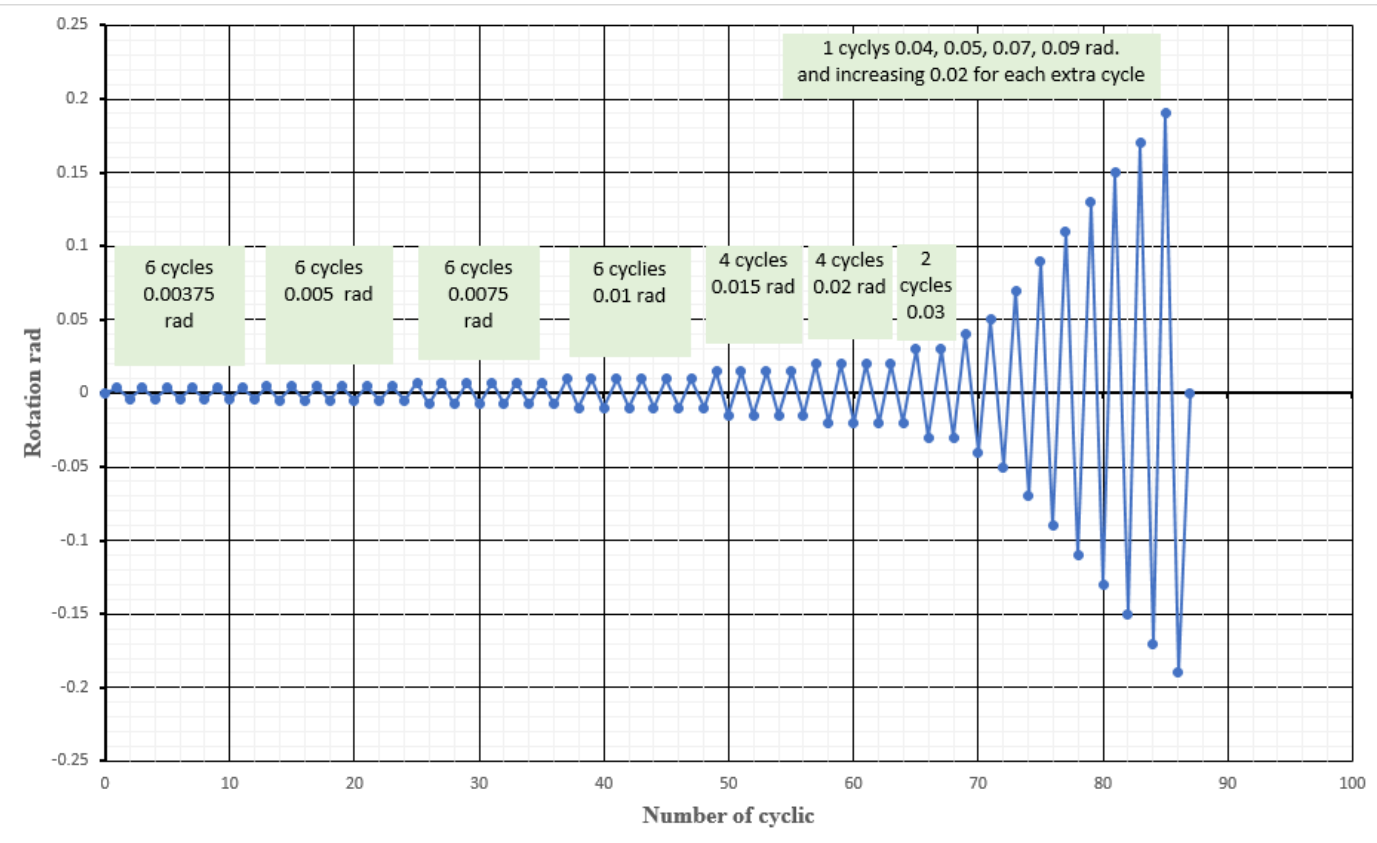

Figure 2.8 Loading protocol (AISC Seismic Design Provision)

\subsection{Test Setup}

Full scale T-EBF quasi static tests were investigated experimentally on link-tocolumn connections. The test setup for the T-EBF is shown in Figure (2.9). The EBF was comprised of two columns of W14x 145, connected with pinned base plates. The 2 " thick column base plates were bolted to the strong floor beam of section W14x132 with $11 / 4^{\prime \prime}$ diameter bolts. The clevises and column-based plate were designed as fully pinned to prevent plastic hinges forming in the column. The beams and links were wide flange single continuous member sections of W18x76 and W16x67, specified as A992 grade 50 steel, while all the plates and stiffeners were A572 grade 50. The actuator was mounted horizontally on a reaction frame to impose cyclic load and was connected to the south column. The actuator capacity was $979 \mathrm{kN}$ (220 kips) and had a stroke of $( \pm) 254 \mathrm{~mm}$ 
$\left(10^{\prime \prime}\right)$. It was connected horizontally to the reaction frame at a level of $3446 \mathrm{~mm}\left(135.68^{\prime \prime}\right)$ above the pin-supported column centerline, and the distance between the two pinned columns was $2981 \mathrm{~mm}(117.375 ")$. Figure (2.10) shows a more detailed overview of the test setup.

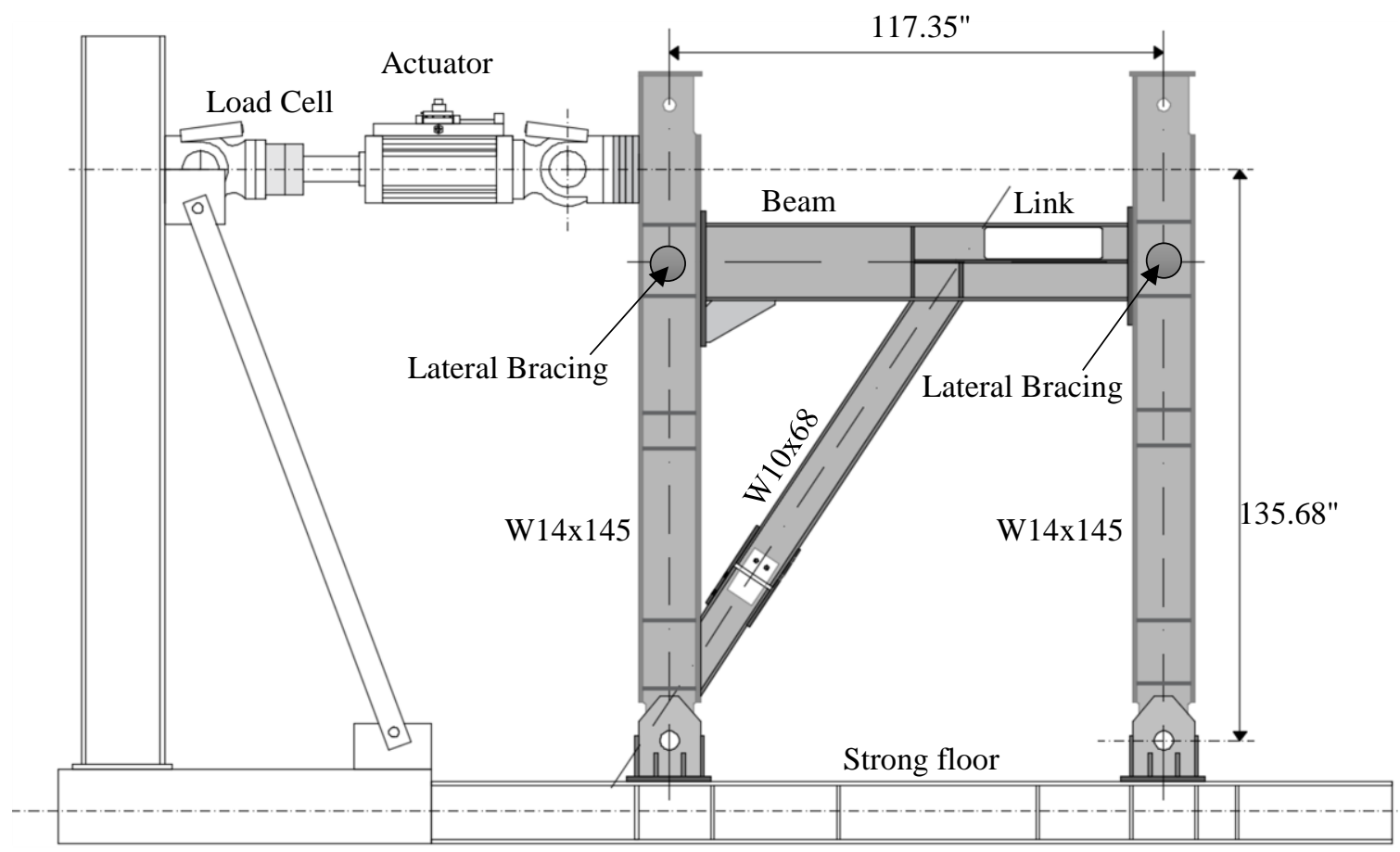

a) EBF welded braced 


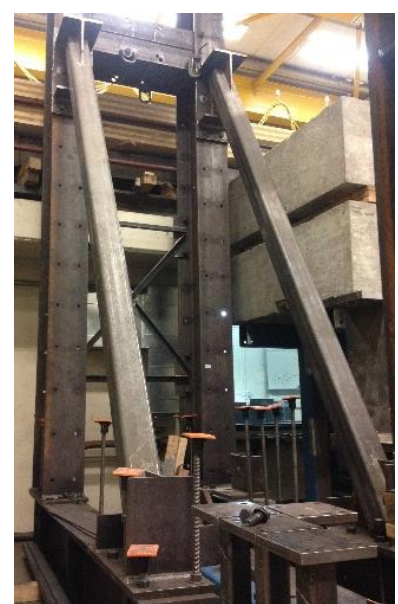

b) Reaction frame

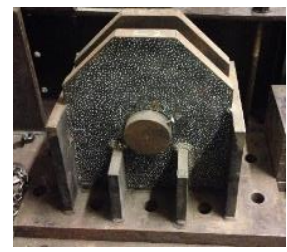

c) Column clevis

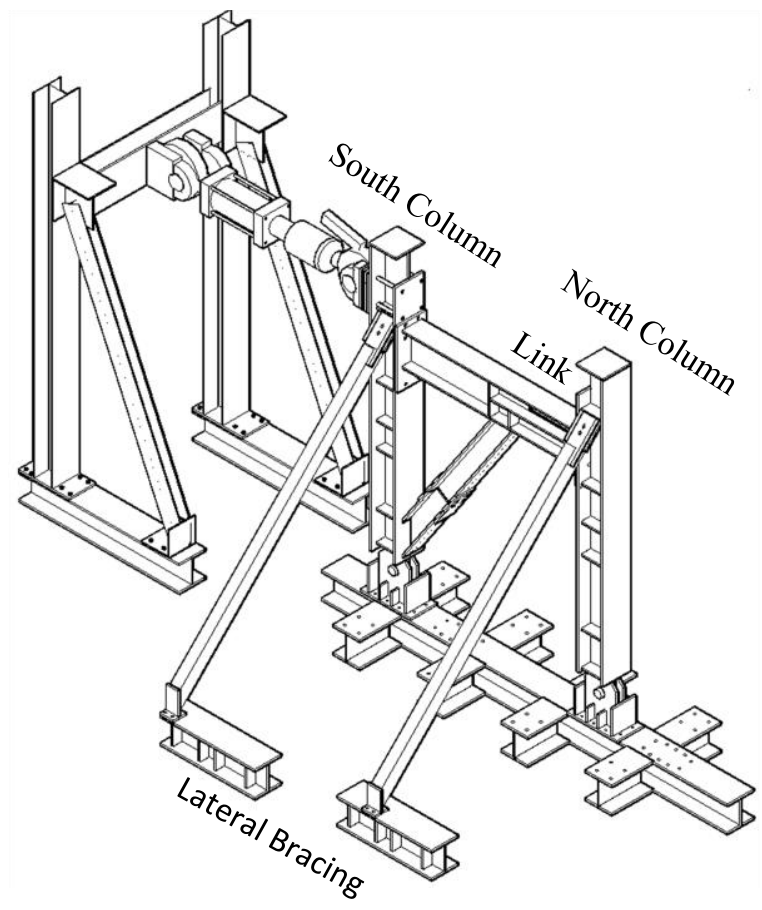

d) 3D test setup

Figure 2.9 Test setup

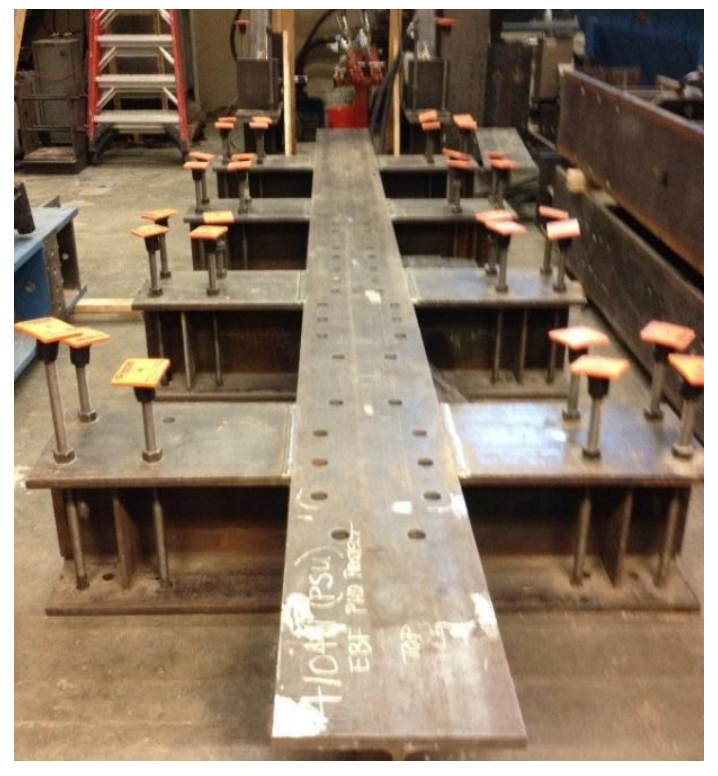

(a) Floor beam

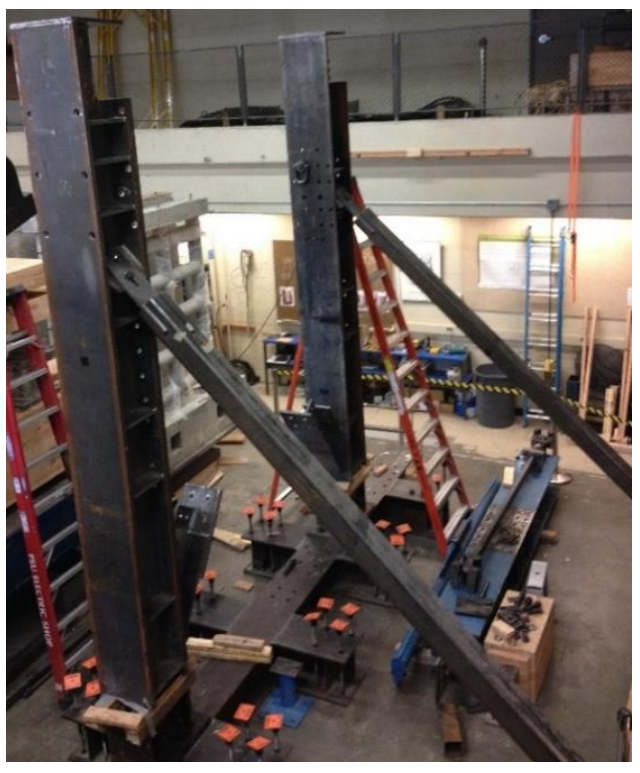

(b) Lateral bracing 


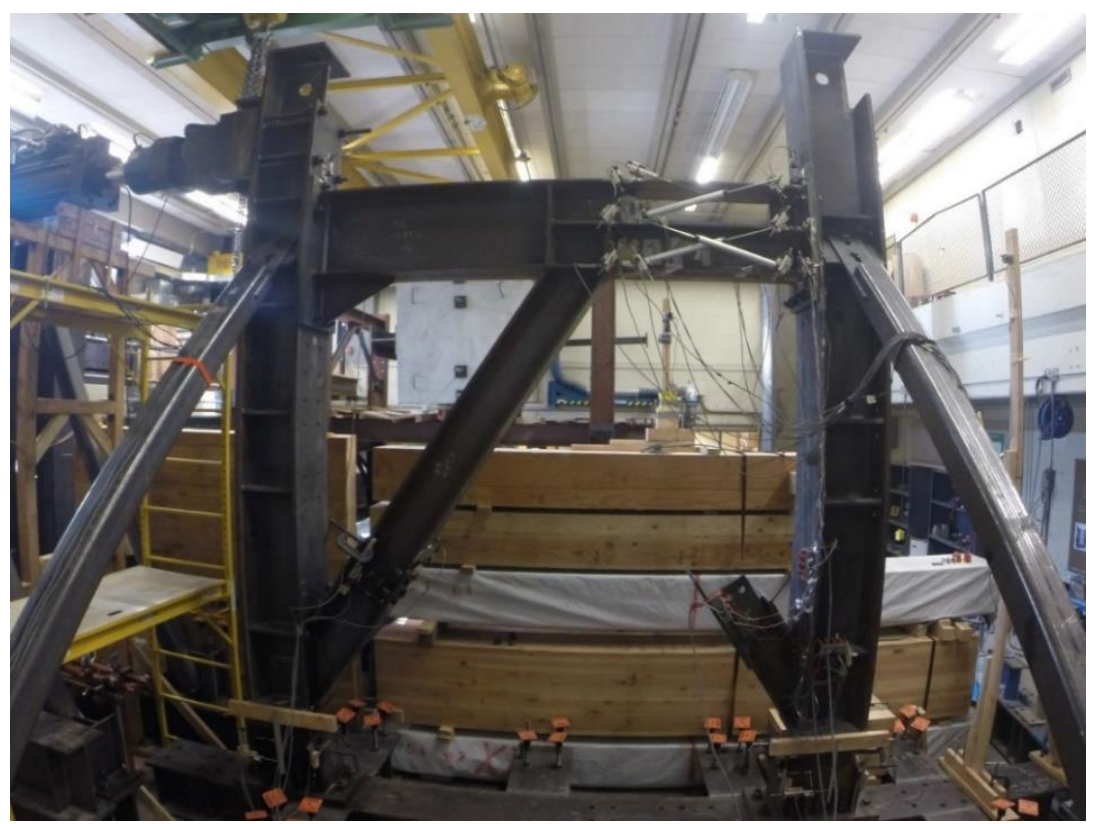

(c) EBF welded brace before testing

Figure 2.10 Overall view of the test setup

\subsubsection{Columns}

The two columns of section W14x145, the floor beam, and the clevises used in this research were left from previous tests (Volynkin , 2016). The column details are shown in Figure (2.11). The south column was connected to the actuator, while the north column was connected to the specimens through the end plate. Six bolts of $11 / 8$ " diameter (group B) and 2 bolts of 1.5" diameter (group A) were used in the end plate of the beam and the south column. In the other side, ten bolts of $11 / 8^{\prime \prime}$ diameter (group B) and two bolts of $1.25^{\prime \prime}$ diameter (group B) were used to connect the link with the north column. The location of end plates and bolts used in the tests are shown in the figure below. 


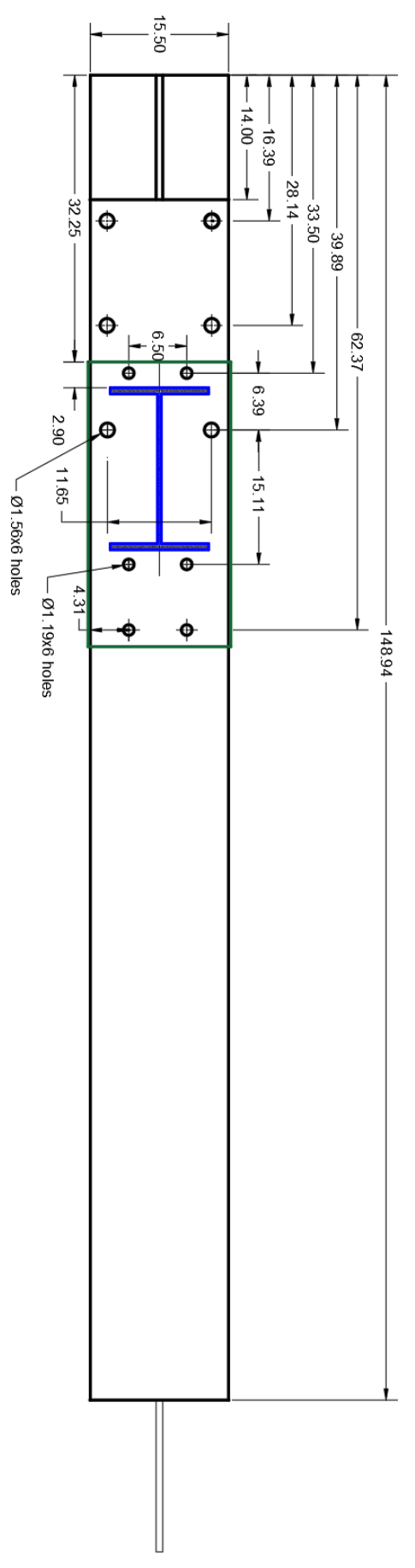

(a) South column

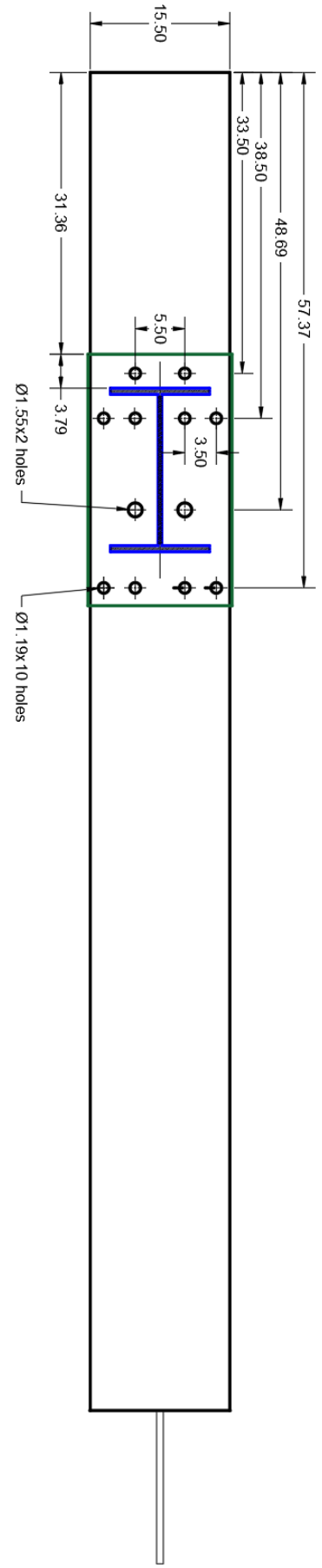

(b) North column

Figure 2.11 Columns details with used bolts and endplates locations 


\subsection{Test Phases and Specimens Description}

All the detailed design and fabrication drawings are included in the Appendix C. The calculations are also attached in Appendix A. In general, for all specimens, the links were welded with a $1 / 2$ " fillet weld to end plates of 1.25 " thickness, made from A572 steel grade 50 . The end plates were bolted to the columns. The bracing member has a section of W10x68. For setup stability, the frame was supported at two points by lateral braces to prevent the out of plane movement of specimens.

\subsubsection{Phase One: T-EBF, Welded Diagonal Brace Connection}

The first phase of T-EBF consisted of two specimens of different sections, W18x76 and W16x67, in which the brace member was welded with the beam. The key dimensions of the specimens are included in Table (2.1).

Table 2.1 Tuned EBF key dimensions, in. (mm)

\begin{tabular}{|c|c|c|c|c|c|c|c|c|c|}
\hline Sections & ED & $\mathbf{t}_{\text {hs }}$ & $\mathbf{t}_{\text {vss }}$ & $\mathbf{e}^{\prime}$ & $\mathbf{e}$ & $\mathbf{h}_{\mathbf{w}}$ & $\mathbf{W}_{\mathbf{s}}$ & $\mathbf{r}_{\text {top }}$ & $\mathbf{r b o t}$ \\
& & & & & & & & & \\
\hline W18x76 & 6 & 0.75 & 0.75 & 27.812 & 39.812 & 8.07 & 0.25 & 0.5 & 0.5 \\
& $(152)$ & $(19)$ & $(19)$ & $(707)$ & $(1011)$ & $(205)$ & $(6.35)$ & $(12.7)$ & $(12.7)$ \\
\hline W16x67 & 6 & 0.75 & 0.75 & 27.25 & 39.25 & 8 & 0.25 & 0.5 & 3 \\
& $(152)$ & $(19)$ & $(19)$ & $(693)$ & $(997)$ & $(203)$ & $(6.35)$ & $(12.7)$ & $(76.2)$ \\
\hline
\end{tabular}

\subsubsection{First Specimen}

Figure (2.12) shows the main parametric dimension used in T-EBF for a W18x76 welded brace. The link was created by making an opening in the web and adding horizontal and vertical stiffeners at the brace connection area. The transition zones in the 
web cutout had a radius of $1 / 2$ ". The length of the opening was selected to provide area for the end panels, which are important to shift the inelastic region away from the filletwelded end plate connection.

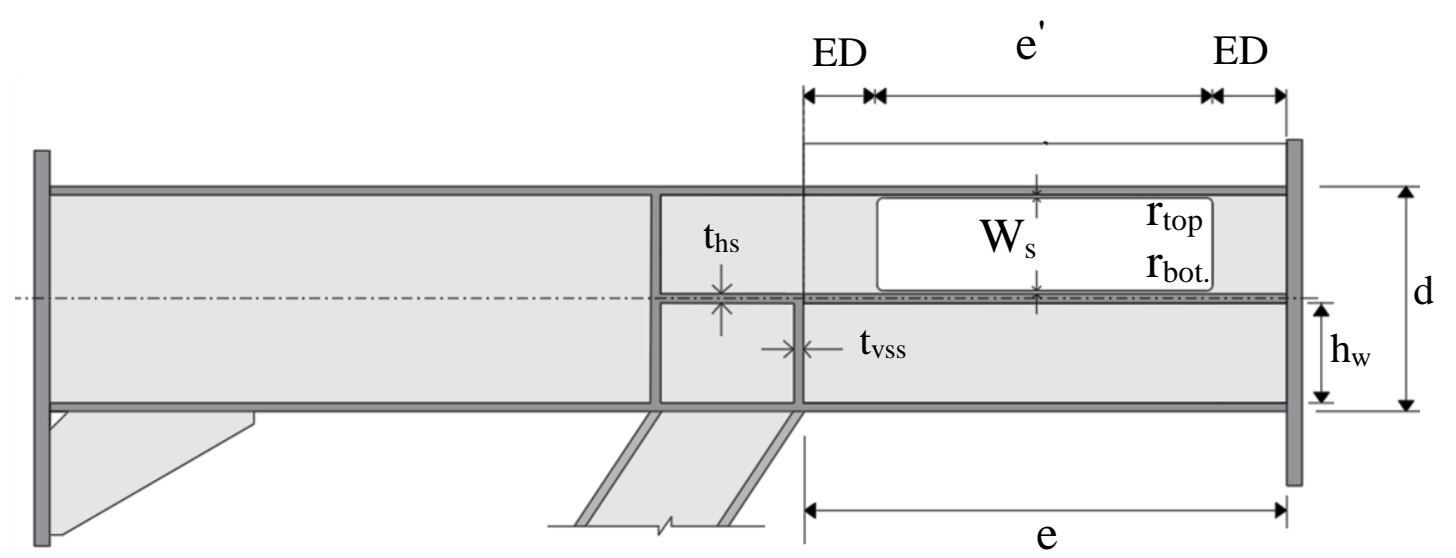

Figure 2.12 Configuration of first specimen

\subsubsection{Second Specimen}

The second specimen was a W16x67 welded brace. The link web opening configuration of this section was modified to prevent the cracks that appeared in the first section at the radius transition on both north and south sides. The fillet radius was increased from $12.7 \mathrm{~mm}\left(1 / 2^{\prime \prime}\right)$ to $76.2 \mathrm{~mm}\left(3^{\prime \prime}\right)$ for the opening bottom edge, with a $45^{\circ}$ diagonal edge side as shown in Figure (2.13). 


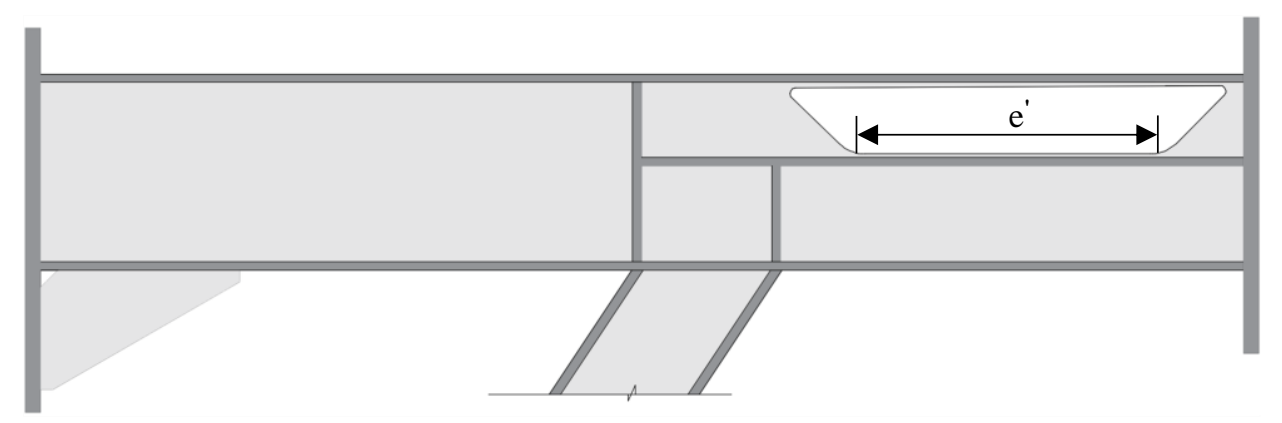

Figure 2.13 Configuration of second specimen

\subsubsection{Phase Two: T-EBF, Bolted Diagonal Brace Connection}

The second phase also comprises two specimens (W18x76 and W16x67) however, the brace was bolted with the beam.

\subsubsection{Third Specimen}

The third specimen had a section of W18x76 with a bolted brace member. The primary purpose of this specimen was to compare the behavior of a bolted brace-to-link connection in T-EBF with the first specimen of welded brace-to-link. Figure (2.14) describes the specimen configuration, which used a thick plate at the bracing connection to support the forces developed. 


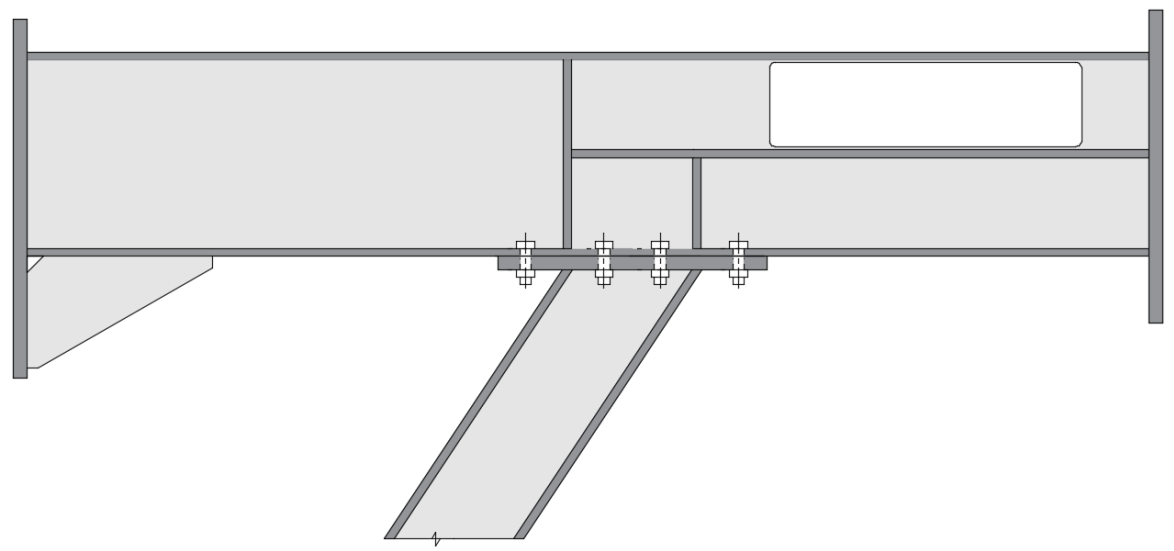

Figure 2.14 Configuration of third specimen

\subsubsection{Fourth Specimen}

Cracks at the transition zone of the third section (W18x76) spread and grew with the increase of the link rotation. Therefore, the last specimen, section W16x67, was supported by four bolts (1" dia.), as shown in Figure (2.15), to control the danger of cracks. Table (2.2) shows the properties of the whole threaded high strength rod that was used in the fourth specimen.

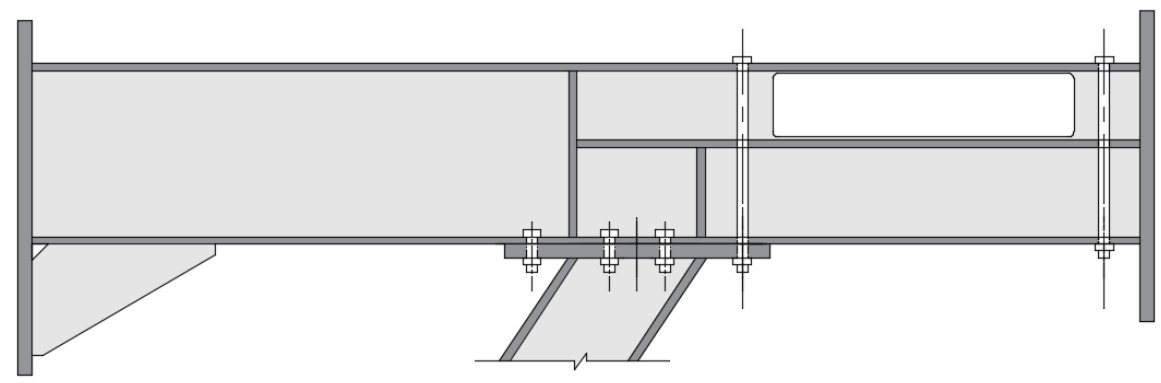

Figure 2.15 Configuration of fourth specimen 
Table 2.2 Threaded rod properties

\begin{tabular}{|c|c|c|c|}
\hline $\begin{array}{c}\text { Dia./Threaded size } \\
\text { inch }\end{array}$ & Tensile strength & Length & Material grade \\
& psi & Ft. & \\
\hline $1 "-8$ & 125,000 & 3 & B7 \\
\hline
\end{tabular}

The initial post-tension forces, before applying the lateral loads, were 14.3 kips in average of the four bolt rods. The lateral load was applied according to the AISC loading protocol.

The first phase focused on tuning the link in EBF and checking the stability of the frame to achieve the target seismic requirements. The second test phase focused on the brace to link connection in T-EBF, in keeping with the objective of link and beam replaceability due to either beam/link fracture or to repeated earthquakes during the design level of the frame.

The details of the exact design drawing that were sent to the fabricator are included in appendix C.

\subsection{Materials}

The beams and links of sections W18x76 and W16x67 were made from the same material: A992 steel. The measured dimensions of the sections are presented in Table (2.3), as compared with the design dimensions. 
Table 2.3 Dimensions of link sections (in)

\begin{tabular}{|c|c|c|c|c|c|}
\hline Sections & Dimensions (in) & d & $t_{w}$ & $b_{f}$ & $\mathrm{t}_{\mathrm{f}}$ \\
\hline \multirow[t]{2}{*}{ W18x76 } & Measured & 18.2 & 0.403 & 11.0 & 0.642 \\
\hline & Nominal & 18.2 & 0.425 & 11.0 & 0.680 \\
\hline \multirow[t]{2}{*}{ W16x67 } & Measured & 16.3 & 0.392 & 10.2 & 0.634 \\
\hline & Nominal & 16.3 & 0.395 & 10.2 & 0.665 \\
\hline \multirow[t]{2}{*}{ Stiffeners } & Measured & - & - & - & 0.738 \\
\hline & Nominal & - & - & - & 0.750 \\
\hline
\end{tabular}

The tensile coupon test results are shown in Table (2.4). The locations of the samples represent the web and flanges of the two specimen sections as well as the horizontal stiffeners.

Table 2.4 Tension test results

\begin{tabular}{|c|c|c|c|c|}
\hline Sections & Location & $\begin{array}{c}\text { Tensile Strength } \\
\mathrm{ksi}\end{array}$ & $\begin{array}{c}\text { Yield Strength } \\
\mathrm{ksi}\end{array}$ & $\begin{array}{c}\text { Elongation } \\
\text { in 2" (\%) }\end{array}$ \\
\hline \multirow{2}{*}{ W18x76 } & Web & 81.3 & 59.5 & 30.5 \\
\cline { 2 - 5 } & Flange & 77.4 & 57.2 & 35.5 \\
\hline W16x67 & Web & 80.0 & 59.4 & 30.5 \\
\cline { 2 - 5 } & Flange & 77.2 & 56.3 & 34.0 \\
\hline Stiffeners & - & 75.7 & 53.9 & 35.5 \\
\hline
\end{tabular}

The section strength was calculated based on nominal and measured dimensions and tensile strength. Table (2.5) illustrates the values of plastic shear and moment strength for both sections, W18x76 and W16x67. 
Table 2.5 Section properties

\begin{tabular}{|c|c|c|c|c|c|}
\hline \multicolumn{3}{|c|}{ W18x76 } & \multicolumn{3}{c|}{ W16x67 } \\
\hline Strength & $\begin{array}{c}F_{y}=50 k s i \\
(\text { Nominal })\end{array}$ & $\begin{array}{c}F_{y}=59.5 k s i \\
\text { (Measured) }\end{array}$ & Strength & $\begin{array}{c}F_{y}=50 k s i \\
\text { (Nominal) }\end{array}$ & $\begin{array}{c}F_{y}=59.5 \text { ksi } \\
\text { (Measured) }\end{array}$ \\
\hline$V_{p}=0.6 f_{y} A_{w}$ & $\begin{array}{c}119.085 \\
\text { kips }\end{array}$ & $\begin{array}{c}135.46 \\
\text { kips }\end{array}$ & $V_{p}=0.6 f_{y} A_{w}$ & $\begin{array}{c}109.3 \\
\text { kips }\end{array}$ & $\begin{array}{c}128.82 \\
\text { kips }\end{array}$ \\
& & & & & \\
\hline$M_{p}=f_{y} Z$ & 618.334 & 695.455 & $M_{p}=f_{y} Z$ & 508.43 & 589.12 \\
& kips.ft & kips.ft & & kips.ft & kips.ft \\
& & & & & \\
\hline
\end{tabular}

Each test requires using a lot of bolts. Bolt details are described in Table (2.6). Nuts type were hex nut-heavy with nut standard ASTMA194 and material grade 2H right hand. There were no signs of bolt failures during the tests. Figure (2.16) shows the bolts used in one test after the test.

Table 2.6 Bolts properties

\begin{tabular}{|c|c|c|c|}
\hline $\begin{array}{c}\text { Dia./Threaded size } \\
\text { (inch) }\end{array}$ & $\begin{array}{c}\text { Tensile strength } \\
(\mathrm{psi})\end{array}$ & $\begin{array}{c}\text { Length } \\
\text { (inch) }\end{array}$ & Standards \\
\hline $1{ }^{\prime \prime}-8$ & 115,000 & 36 & B7 \\
\hline $11 / 8^{\prime \prime}-7$ & 150000 & $4-1 / 2$ & ASTM F3125 \\
\hline $11^{\prime \prime}-8$ & 150000 & $4 "$ & ASTM F3125 \\
\hline $11 / 4 "-7$ & 150000 & $3-1 / 4^{\prime \prime}$ & ASTM F3125 \\
\hline $11 / 2 "-6$ & 105000 & $4-1 / 2^{\prime \prime}$ & ASTM 563 \\
\hline
\end{tabular}



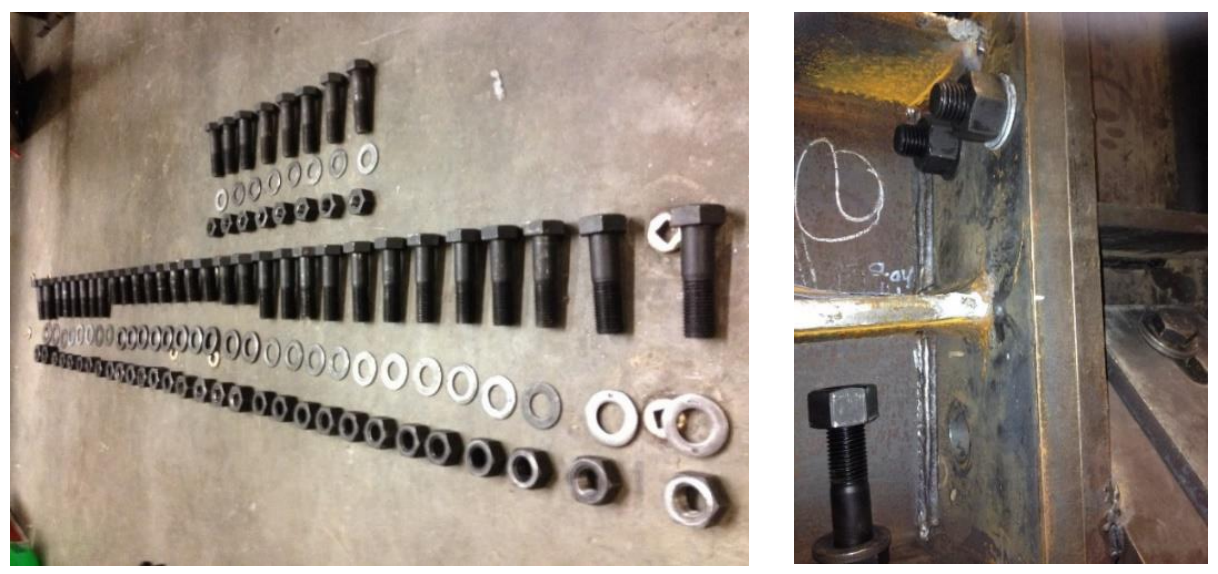

Figure 2.16 Bolts after one test

There are two end plate configurations in this study, the first one is the one that connect the beam with the column and the other one is connected the link with the column. Their details in the four specimens are the same. They have the same dimensions, thickness and number of bolts. They designed according to the Steel Design Guide 4: Extended End-Plate Moment Connections. The performances of them were excellent. Two LVDTs were placed in contact with end plates. As expected, slip to happen in end plates, however, due to the number of bolts that used, very slightly slips were recorded. Figure (2.17) shows the end plate after the test.

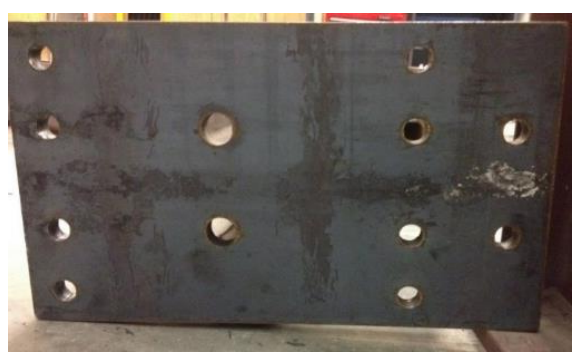

Figure 2.17 End plate after testing 
The brace in this research has two pieces connected using splice. Three LVDTs shown in Figure (2.22d) were used to measure the slips between flanges and web. Even though the slips in this region did not affect on the link behavior, however, the slip data is important in global estimations. The data shows slip did occur at very high loads.

In design of the EBF, the brace should be stay in elastic stage. The Figure (2.18) shows the two bolted braces after the tests. They are both in very good shape and can be used again. The concept of the bolted brace-link connection suggested in this research is showing that the brace can be easily disconnect from the frame and it can be used again which is important in seismic design since the cost and time play an important role in return to occupancy after an earthquake.
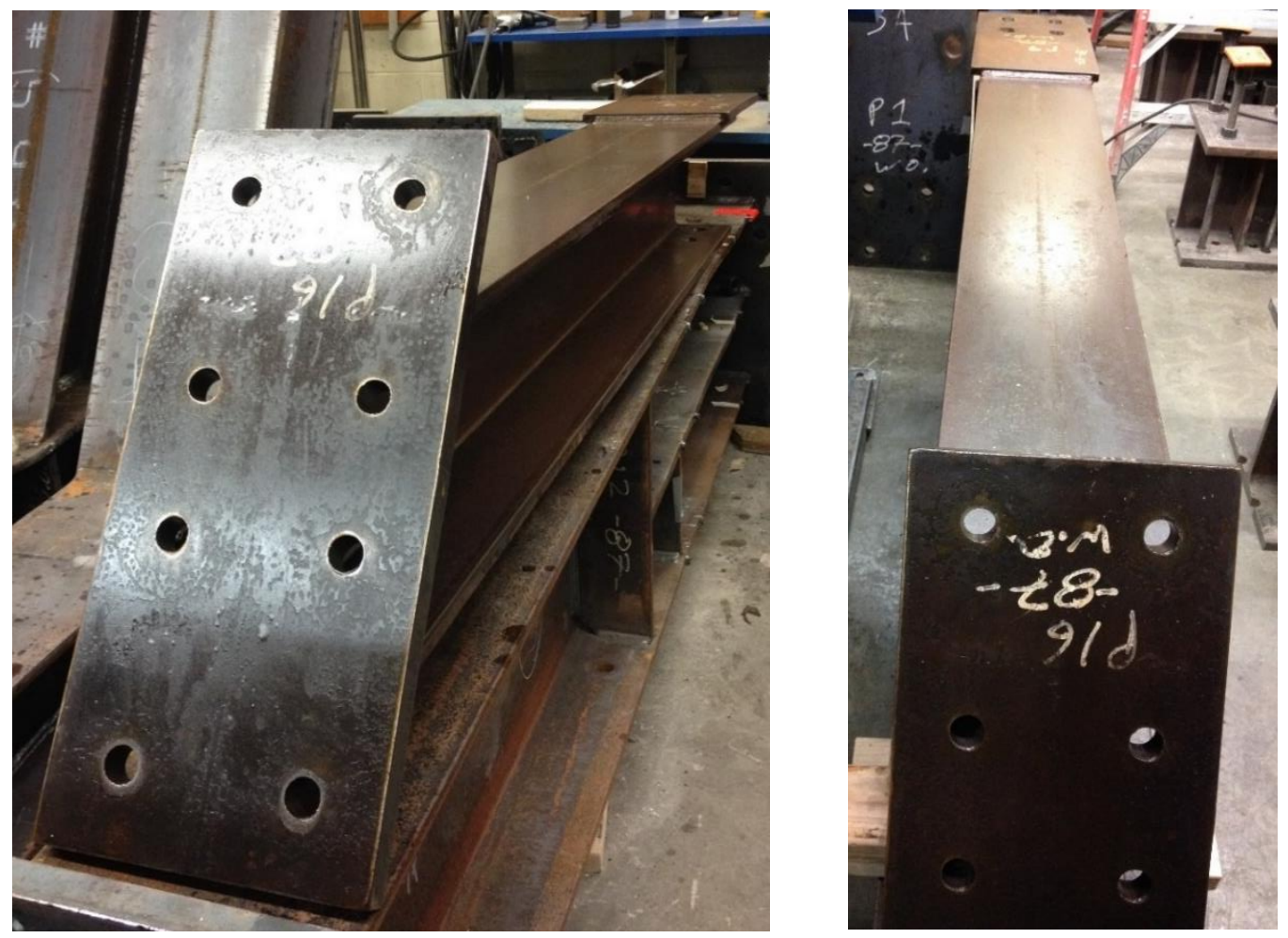

Figure 2.18 Bolted braces after tests 


\subsection{Instrumentation}

Figure (2.19) illustrates the location of instrumentation used within the specimen frame. The cyclic response of T-EBF was measured using LVDTs, strain gages, load cells, and string pots. LVDTs were used to measure displacement, slip and rotation. The string pots were attached to the north column to measure frame drift.

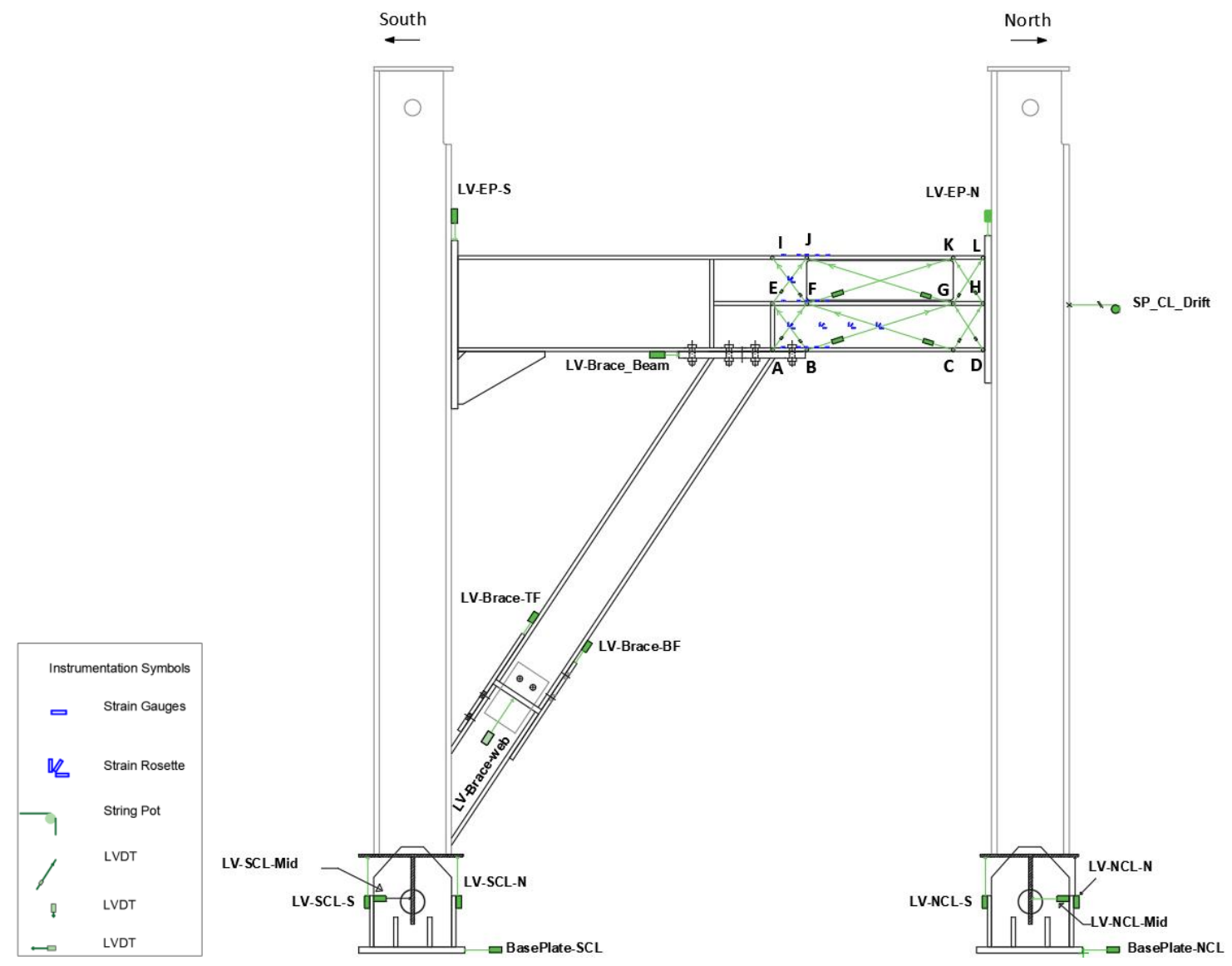

Figure 2.19 Instrumentations layout used on the EBF 


\subsubsection{Strain Gauges}

Two types of strain gauges were used to measure the strains and shears of the specimens. Uniaxial gauges were placed on the link flanges at the most critical location, the opening edge near the bracing member side, where high shear and moment were expected. They were attached on the outer fibers of the top flange and on top of the middle flange to measure the strain along the flanges. Five rosette strain gauges were attached on the link web: three on the effective length panel at mid height, and two glued on the mid depth of the ABEF and EFIJ panels. Rosette gauges were used on the link web to calculate the shear strain developed within. The uniaxial gauges used in this research were YFLA-5-5L (gauge length: 5mm), while the rosette gauges were YEFRA-5, as shown in Figure (2.20) (gauge length: $5 \mathrm{~mm}$ ). Both were from Tokyo Sokki products. Figure (2.21) shows the exact locations of the strain gauges within the all four links.
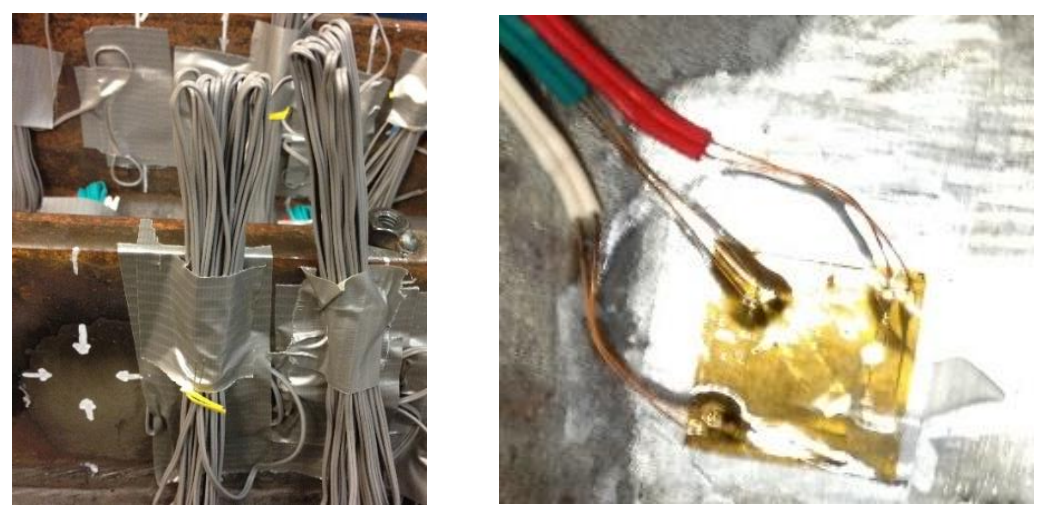

Figure 2.20 Strain gauges 

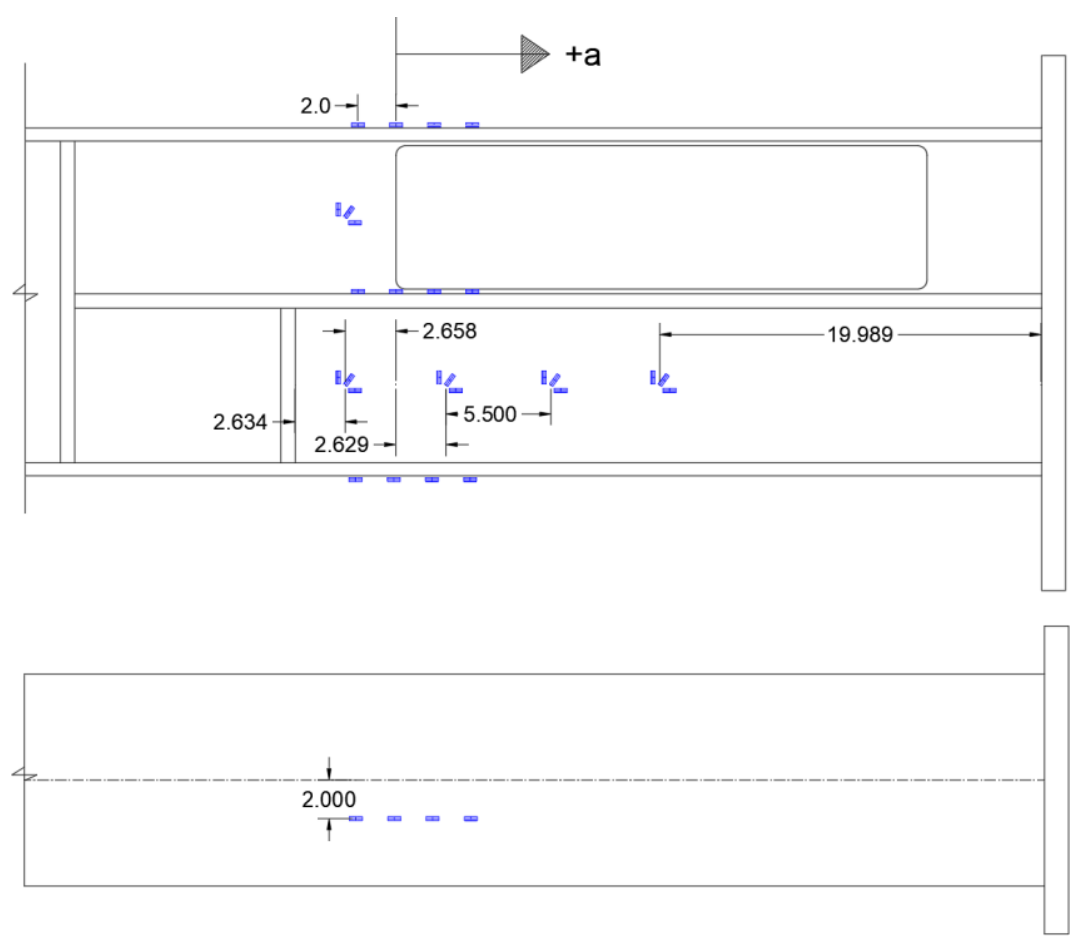

a) W18x76 welded

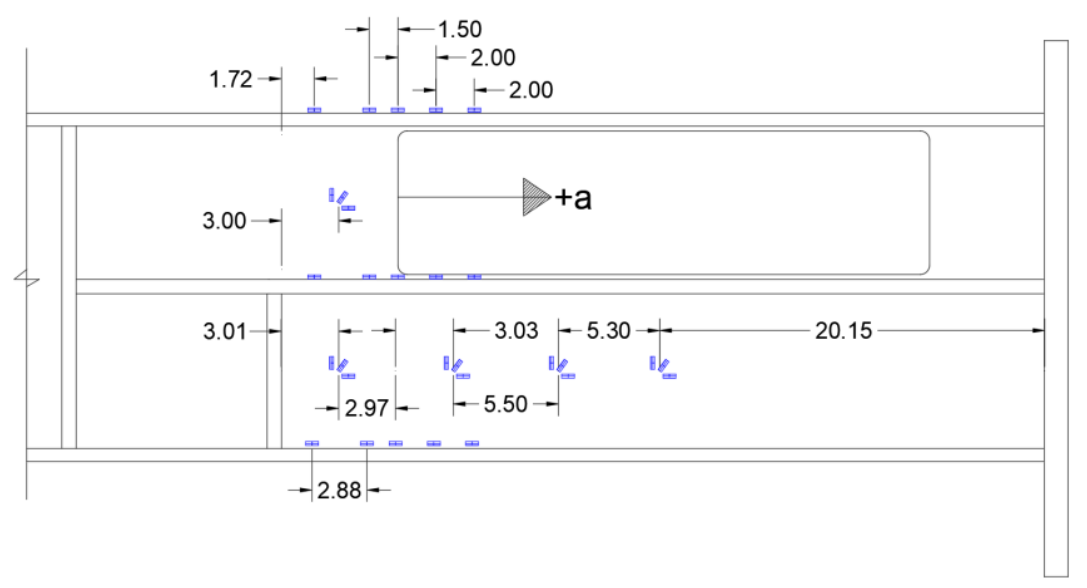

b) W18x76 bolted 


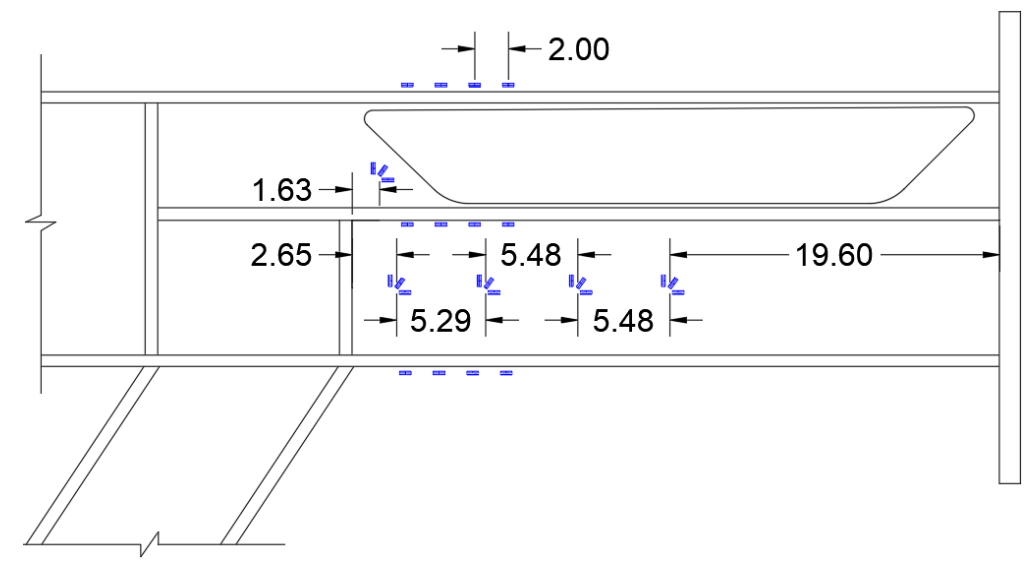

c) W16x67 welded

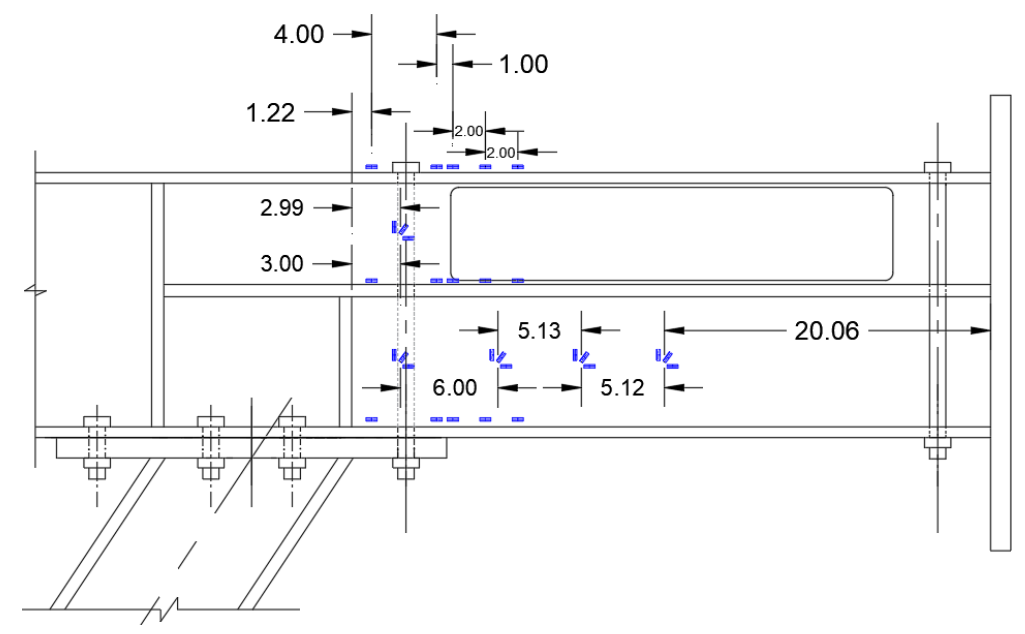

d) W16x67 bolted

Figure 2.21 Strain gages used on the links

\subsubsection{LVDT}

Figure (2.19) shows the location of the LVDTs used during the tests. In addition to the twelve LVDTs used to measure panel rotation within the link, more LVDTs were added to monitor any slip that may occur in the column's base plates, end plates, brace- 
link connection plate, brace flanges, and web. Tack weld was installed at the specific locations on the link flanges in which threaded 5/16" diameter rods were placed to carry the LVDTs. In other areas, C-clamps were used to hold the LVDTs, as shown in Figure (2.22). Six LVDTs were added to the two clevises, three on each, to investigate the movements of the clevises related to the columns. Table (2.7) illustrates in detail the function of each LVDT.

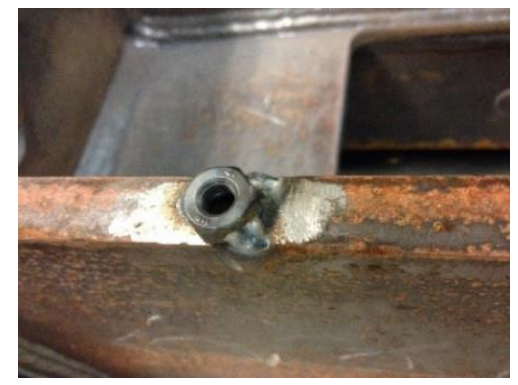

a) Tack welded
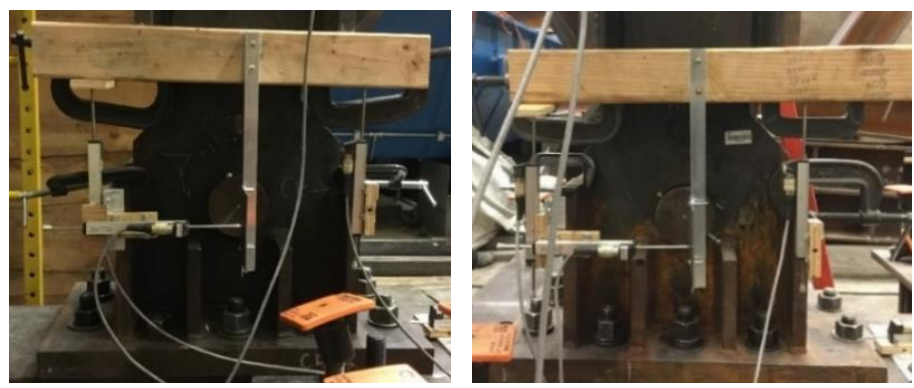

b) South clevis

c) North clevis

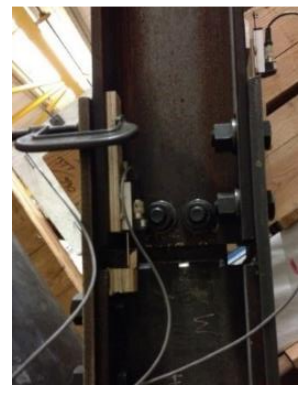

d) Brace member

Figure 2.22 LVDT used on different parts 
Table 2.7 LVDT function

\begin{tabular}{|c|c|c|}
\hline Item & LVDTs Labels & Function \\
\hline 1 & $\mathrm{ABEF}$ & Measuring ABEF panel Rotation \\
\hline 2 & $\mathrm{BCFG}$ & Measuring BCFG panel Rotation \\
\hline 3 & $\mathrm{CDGH}$ & Measuring CDGH panel Rotation \\
\hline 4 & EFIJ & Measuring EFIJ panel Rotation \\
\hline 5 & FGJK & Measuring FGJK panel Rotation \\
\hline 6 & GHKL & Measuring GHKL panel Rotation \\
\hline 7 & LV-EP-N & Link end plate slip, north side \\
\hline 8 & LV-EP-S & Link end plate slip, south side \\
\hline 9 & SP_CL_Drift & North column drift \\
\hline 10 & LV-Brace_Beam & Brace bolted plate slip \\
\hline 11 & LV-Brace-TF & Brace top flange slip \\
\hline 12 & LV-Brace-BF & Brace bottom flange slip \\
\hline 13 & LV-Brace-web & Brace web slip \\
\hline 14 & LV-SCL-N & South clevis vertical movement in north side \\
\hline 15 & LV-SCL-S & South clevis vertical movement in south side \\
\hline 16 & LV-SCL-Mid & South clevis horizontal movement in the middle. \\
\hline 17 & LV-NCL-N & North clevis vertical movement in north side \\
\hline 18 & LV-NCL-S & North clevis vertical movement in south side \\
\hline 19 & LV-NCL-Mid & North clevis horizontal movement in the middle. \\
\hline 20 & BasePlate-SCL & South base plate slip \\
\hline 21 & BasePlate-NCL & North base plate slip \\
\hline 22 & Actuator-Disp. & Actuator applied displacement (frame drift) \\
\hline
\end{tabular}




\subsubsection{Load Cells}

Figure (2.23) shows the locations of load cells used on the last specimens. Four load cells were added to determine the applied forces on four high-tension threaded rods. The actuator load cell was used to monitor the lateral applied loads.

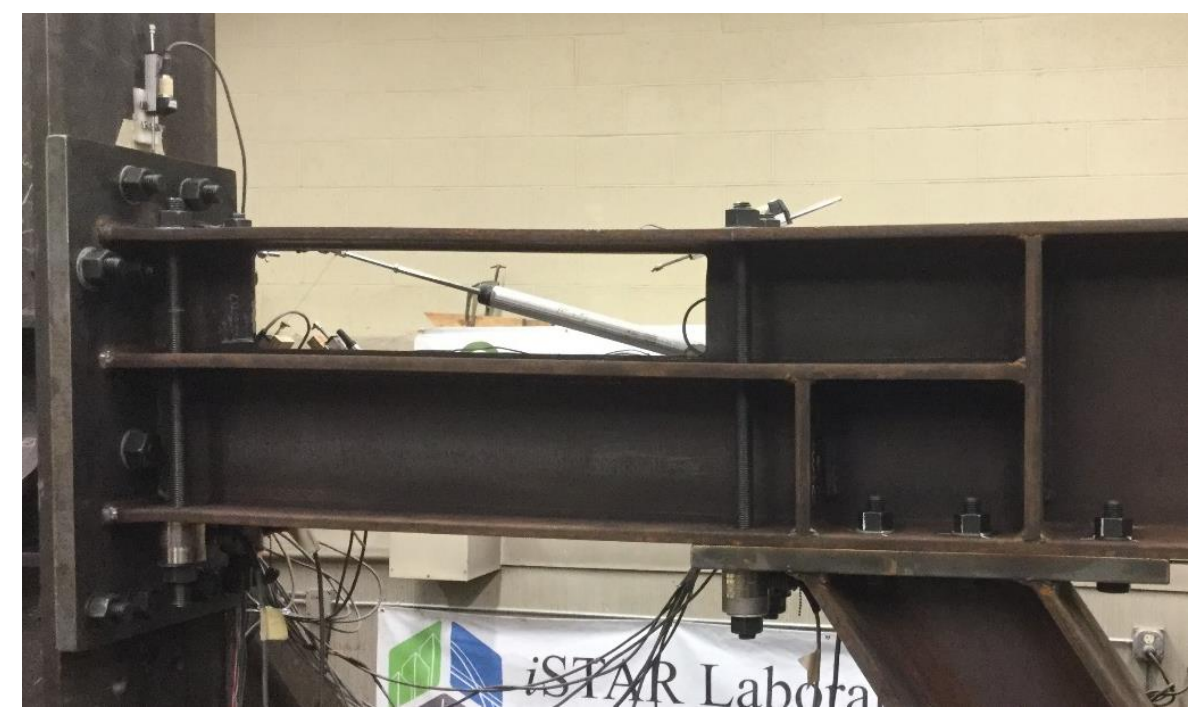

Figure 2.23 Load cell installed on the link

\subsection{Welding Details}

Shop welds were used on all specimens. The specimens were then connected to the other frame parts using bolts. Demand critical welds are defined as welds designated by the engineer and must meet the specific requirement of the Structural Welding CodeSeismic Supplement (AWS) D1.8. Figure (2.24) shows the typical welding details, while the calculations and detailed welding drawings are provided in the appendix A and C respectively. 
All welding sizes are ( 0.5 inches) except as shown

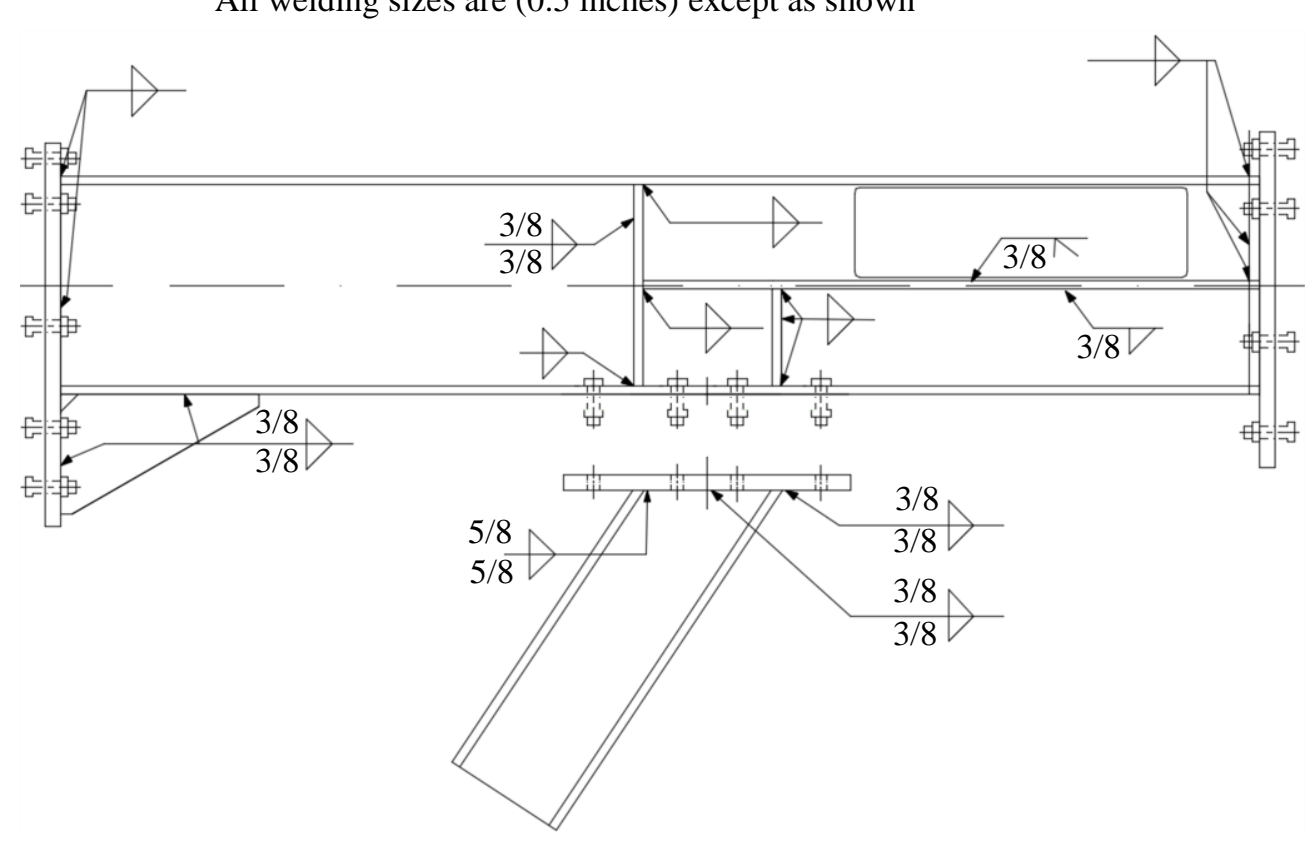

Figure 2.24 Typical welding details

\subsection{Fabrication}

The specimens were fabricated by Fought \& Company Inc, OR. The fabrication drawings are included in Appendix C. 


\section{Chapter 3: Experimental Results}

\subsection{Introduction}

In this chapter, the results from tests will be provided and discussed to assess the performance of T-EBF. The assessments included checking for cracks that might appear in the opening corners at its beginning stages during loading cycles. Seeking for cracks at low link rotation, in which the cracks sizes are small, was difficult since it depends on visual inspections. Therefore, cracks graphs in this chapter, which appear that cracks start at link rotation $0.02 \mathrm{rad}$, they might occur at earlier stages. The main findings from this chapter are the link shear rotation relation, strains and shear strains within the links, panels' rotations, cracks and comparison between the specimens of both major cases welded brace and bolted brace.

\subsection{Results Discussion}

The sign convention in this study is illustrated in Figure (3.1). A clockwise rotation is a positive rotation, while the counterclockwise is a negative rotation. Strain and shear strain profiles are plotted against the distance from the start of the link effective length, denoted a, see Figures (2.21a and b) and (3.1) for clarifying the distance a.
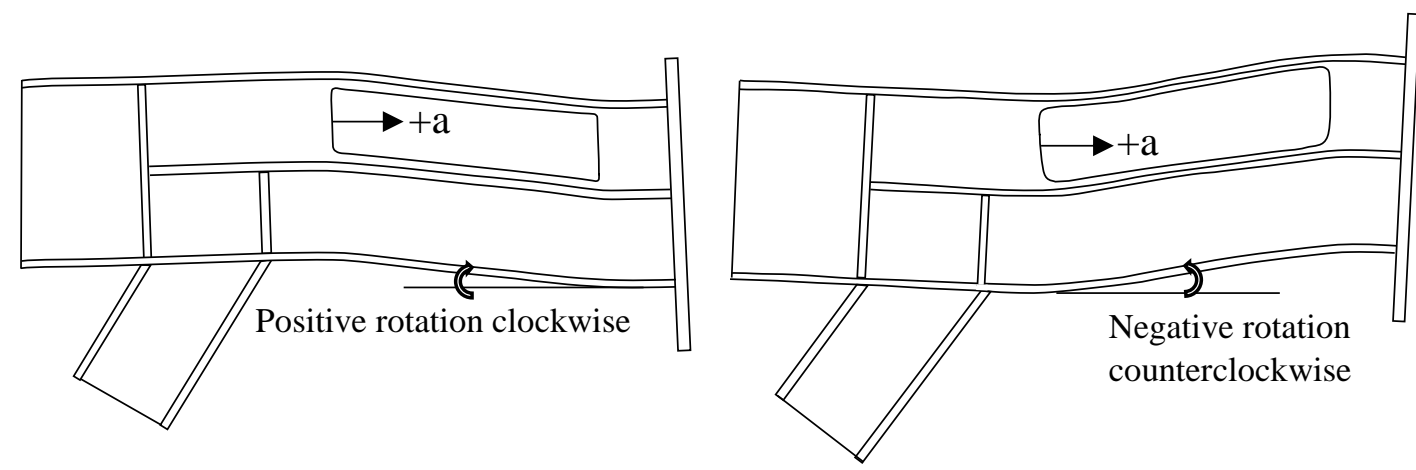

Figure 3.1 Rotation sign conventions 


\subsubsection{Hysteretic Behavior}

Hysteresis curves were plotted for all specimens to assess the cyclic response of the T-EBF links as shown in Figure (3.2). Achieving the inelastic deformation capacity of the link is one of the most significant measurements of the shear link. The tested specimens exceed the minimum rotation of the shear link, $\gamma_{\min }=0.08 \mathrm{rad}$, which is relayed by the seismic design provision (AISC) and all four links showed the desired ductility through the inelastic shearing with the failure mode located inside the link web. The high inelastic rotation capacity of the T-EBF links showed the feasibility of using this type of link to allow control over the link strength properties. The mode failure for both sections was comparable to each other. The first specimen, W18x76 brace welded, reached the actuator capacity at 0.15 rad with no signs of link failure; therefore, the last rotation was again repeated with attention paid to web buckling this time. The second W18x76 bolted specimens achieved up to 0.17 rad before strength degradation was noticed due to web buckling and ending with web fractures. The specimens, W16x67 brace welded, reached rotations of 0.19 rad with shear force approximately equal to the link plastic strength $V_{p}$. In the hysteresis curves of Figure (3.2), a drop in shear capacity indicates the occurrence of web buckling. Figure (3.3) shows the deformed specimens at $0.15 \mathrm{rad}$ of the W18x76 welded and bolted brace. Local buckling and the fracture for that specimens are also shown in Figure (3.3). For both sections, there was no lateral torsional buckling to be considered. No failure of bolts or welding occurred during the tests. 


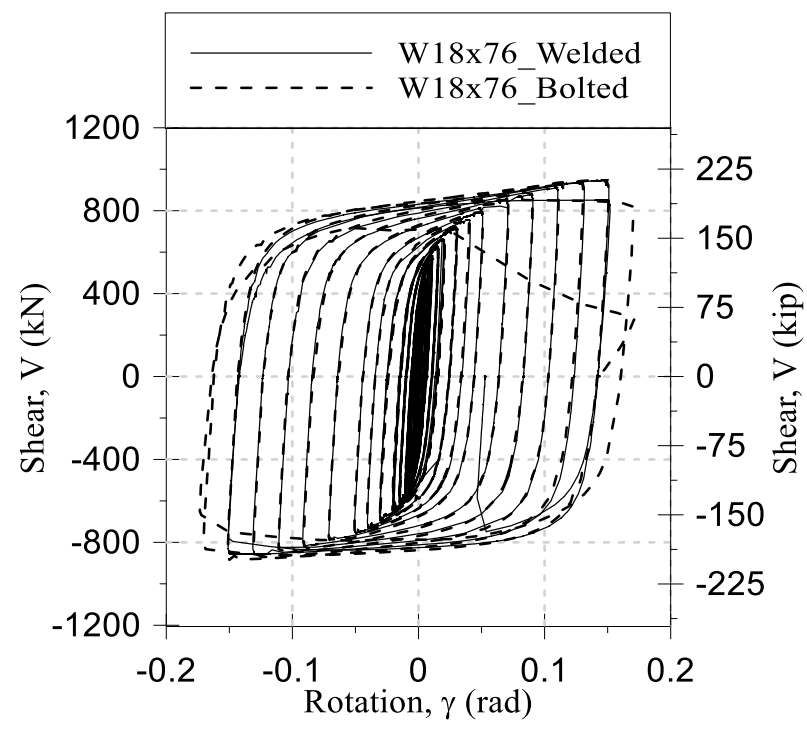

a) W18x76 section

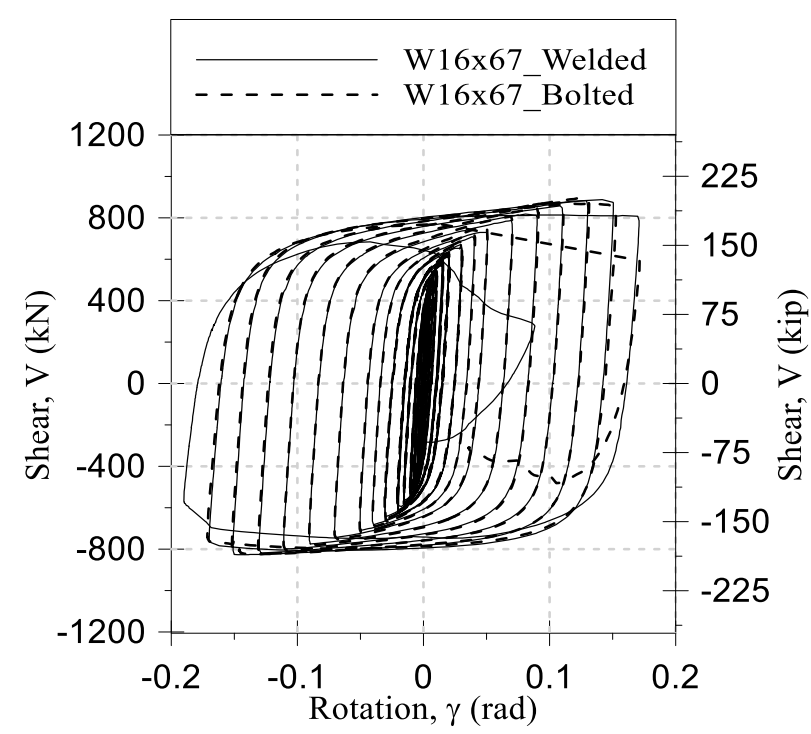

b) W16x67 section

Figure 3.2 Comparison link shear force versus rotation hysteresis curve for both sections. 


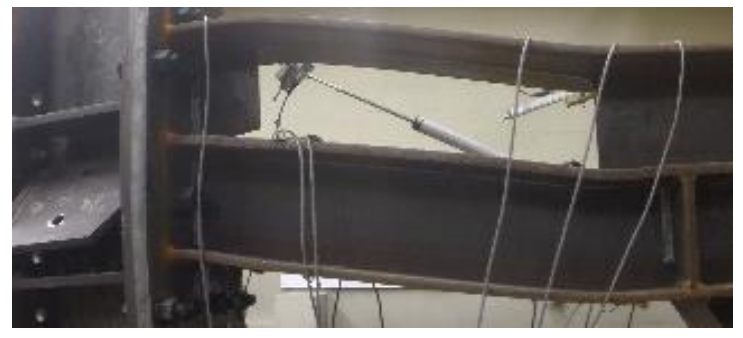

(a) W18x76 Welded at $\gamma=-0.15$

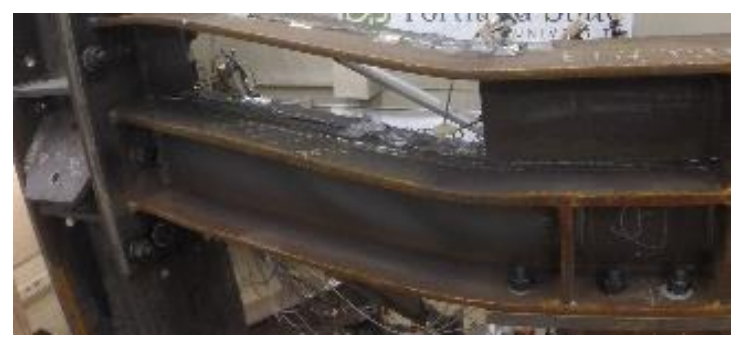

(c) W18x76 Bolted at $\gamma=-0.15$

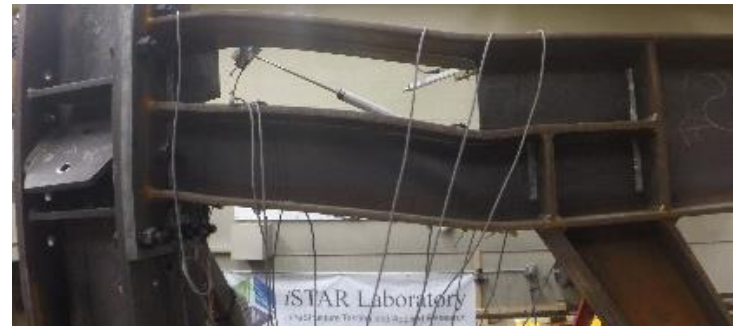

(e) W18x76 Welded at repeated $\gamma=-0.15$

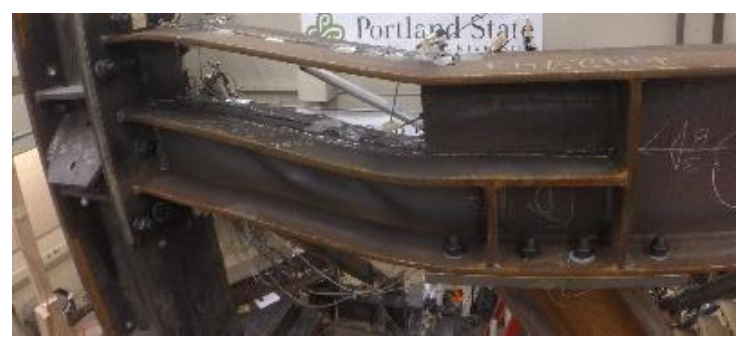

(g) W18x76 Bolted at $\gamma=-0.17$

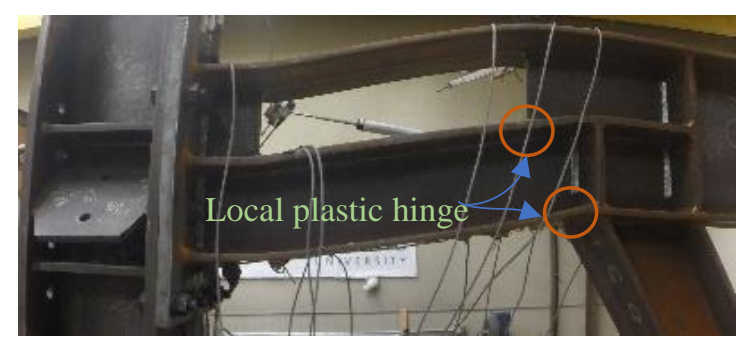

(b) W18x76 Welded at $\gamma=+0.15$

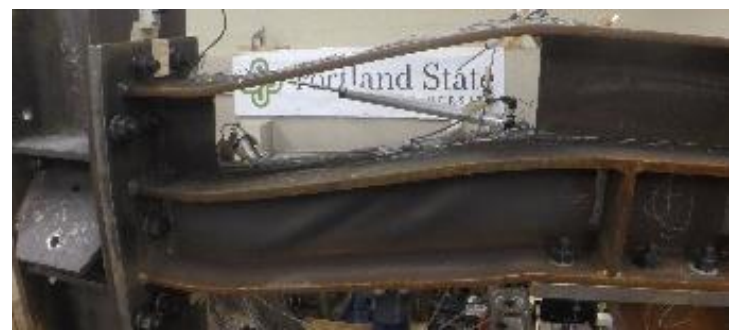

(d) W18x76 Bolted at $\gamma=+0.15$

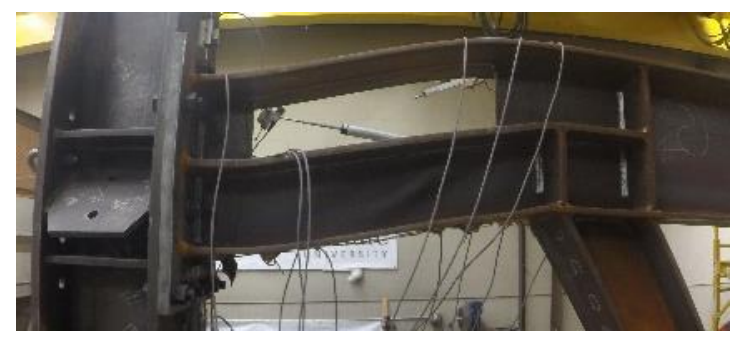

(f) $\mathrm{W} 18 \times 76$ Welded at repeated $\gamma=+0.15$

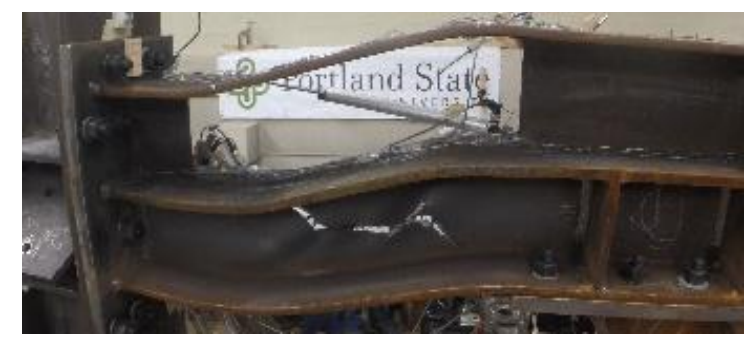

(h) W18x76 Bolted at $\gamma=+0.17$

Figure 3.3 Specimens deformation 
Slight plastic local buckling was observed in many locations along the flanges. The local flange buckling formed in the bottom flange of W18x76 welded near the bracing member and in the middle flange at the opening edge in positive rotation. During negative rotation, it was noticed that the local flange hinge appeared near the end plate. The plastic hinges also generated in the W16x67 welded bottom flange but a little far away from the bracing member. Generating plastic hinges away from the connections protects the welds, bolts, connections, and the bracing member from fracture or failure and the plastic local flange hinges have some effect on the panels' rotation and strain signs, as discussed later. Formatting plastic hinges at reasonable distance can help to reduce the stress level near the column face. There were no effects of flange buckling on the link capacity. Figures (3.4) and (3.5) show the links deformations at different rotation angles for both specimens, W16x67 welded brace and W16x67 bolted brace respectively. 


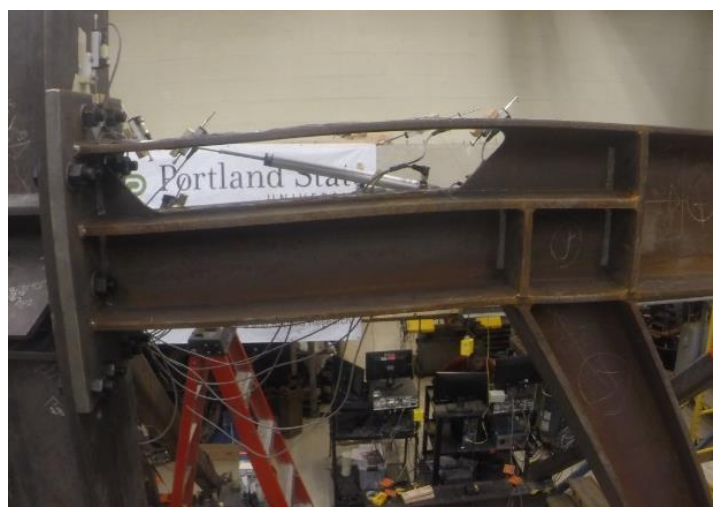

(a) $\gamma=+0.02$

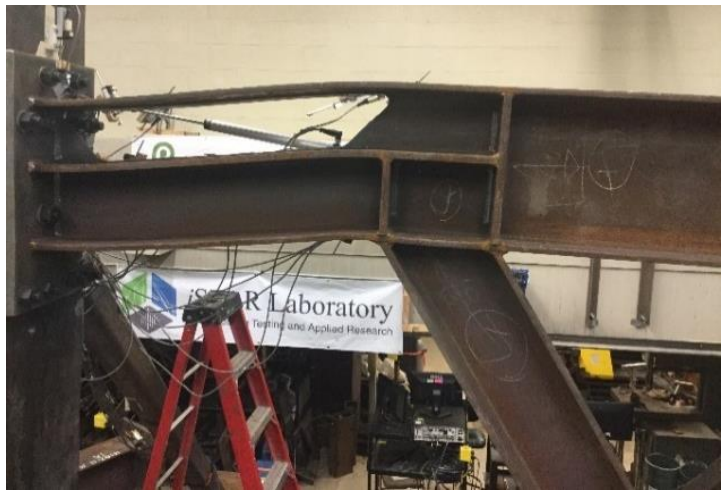

(c) $\gamma=+0.11$

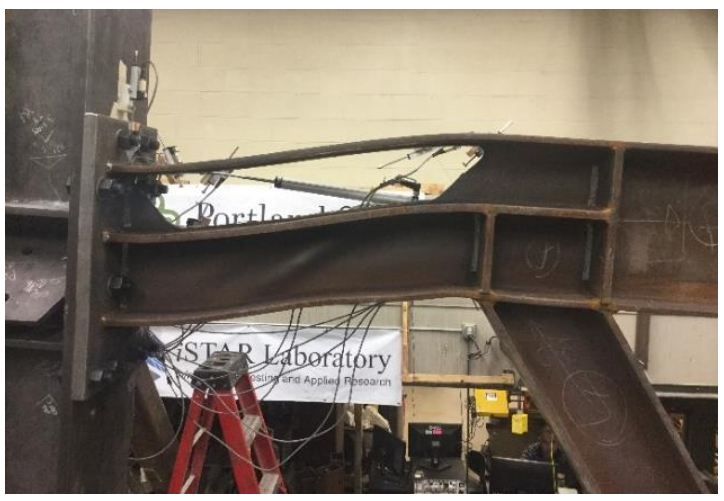

(e) $\gamma=+0.17$

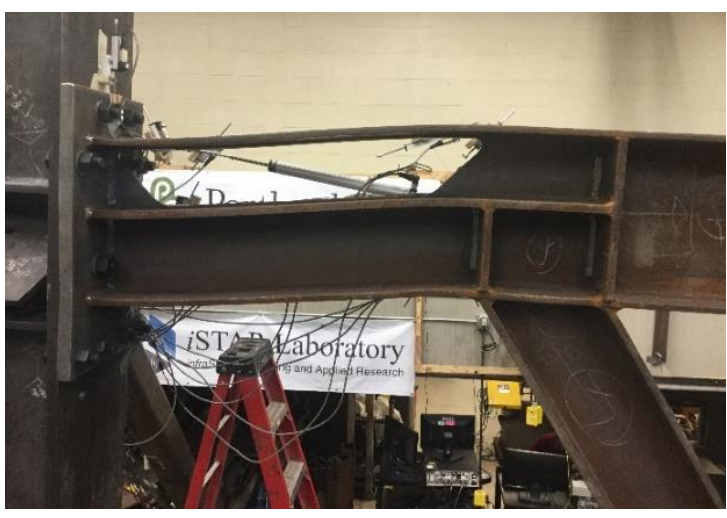

(b) $\gamma=+0.09$

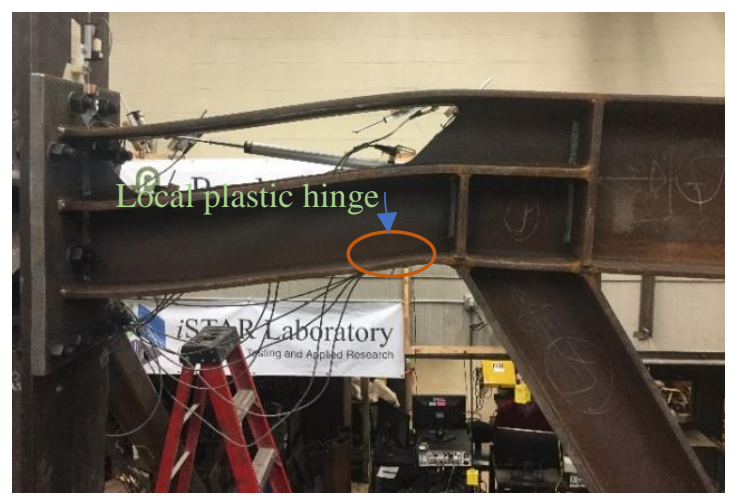

(d) $\gamma=+0.15$

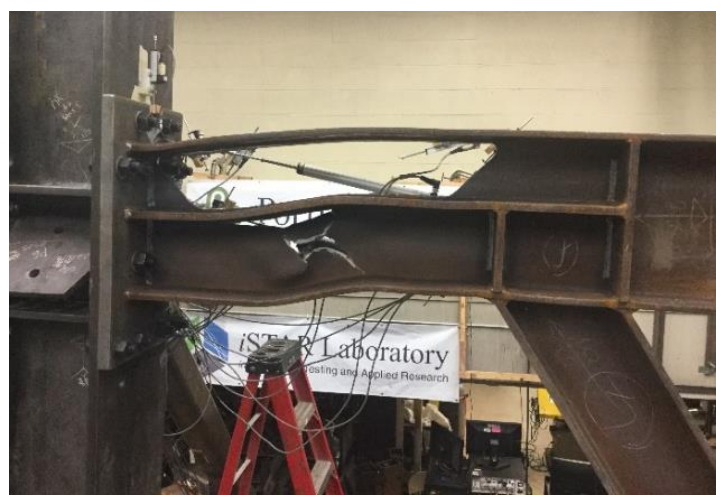

(f) at the final fracture

Figure 3.4 Specimens deformation (W16x67 Welded brace) 

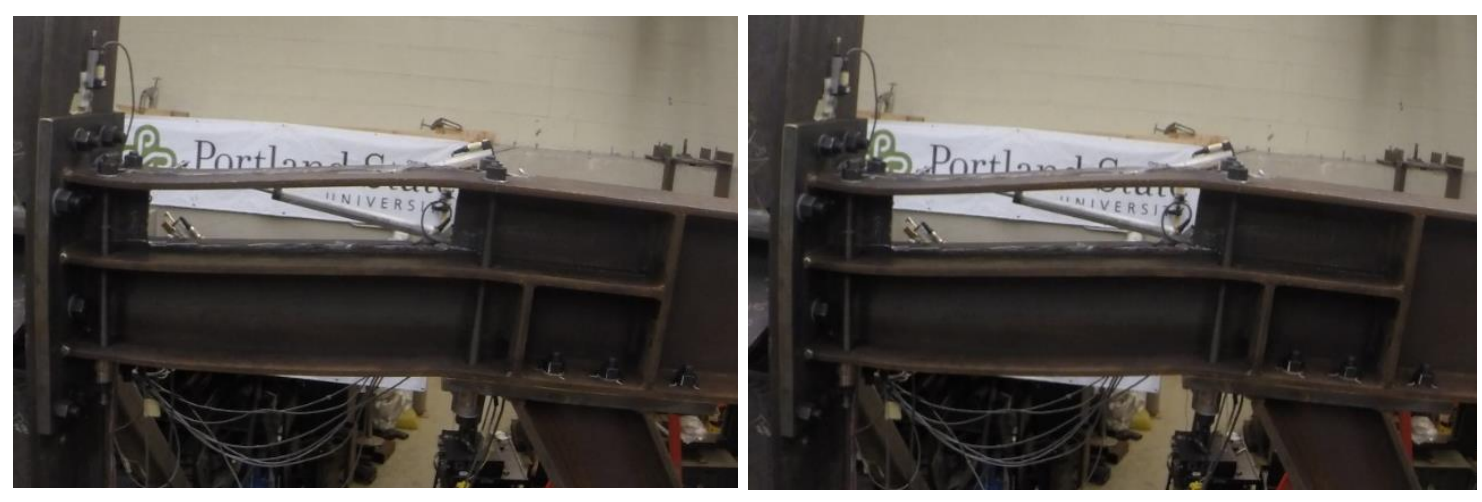

(a) $\gamma=+0.09$

(b) $\gamma=+0.11 \mathrm{rad}$
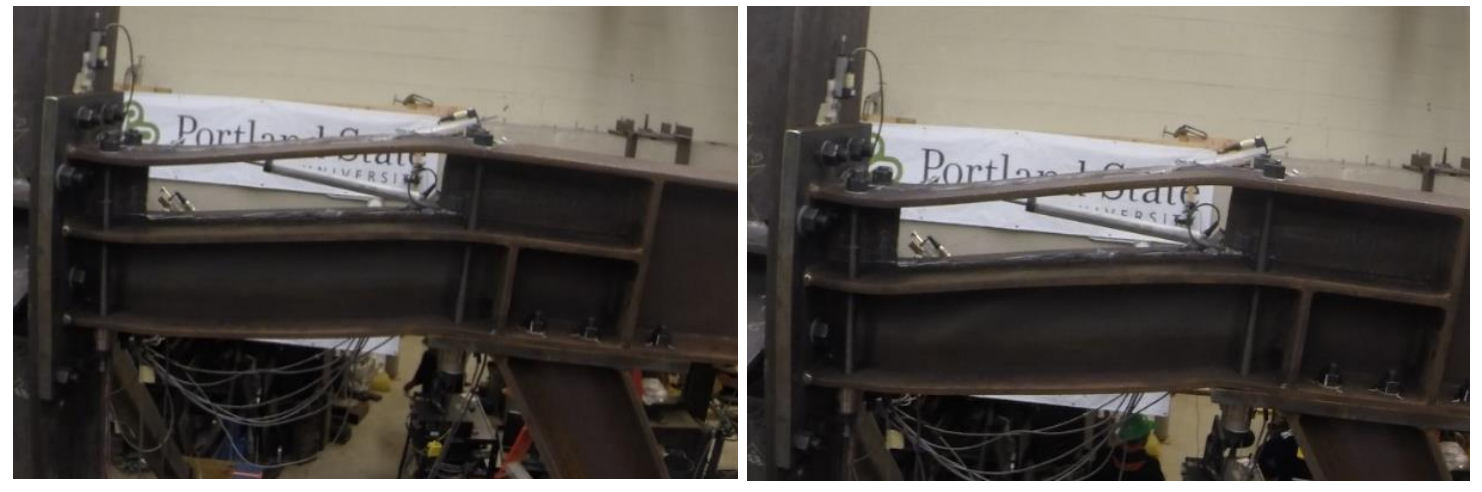

(c) $\gamma=+0.13 \mathrm{rad}$

(d) $\gamma=+0.15 \mathrm{rad}$
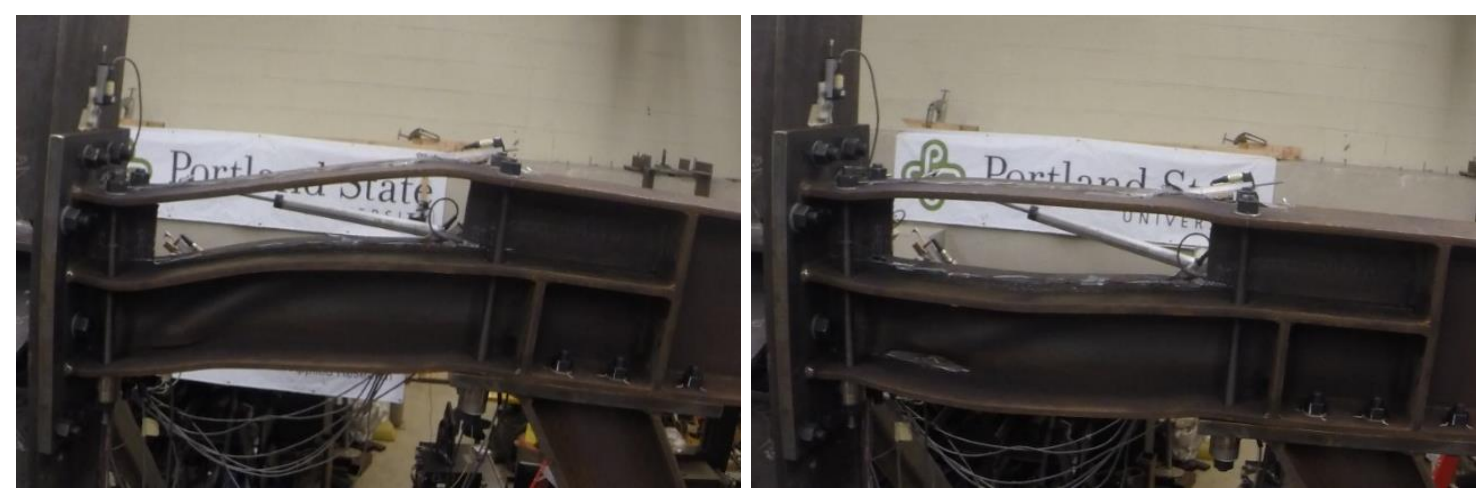

(e) $\gamma=+0.17 \mathrm{rad}$

(f) Link fracture (at zero rotation)

Figure 3.5 Specimens deformation (W16x67 Bolted brace) 


\subsubsection{Shear Strength}

The overstrength factor $\Omega$, which is defined as the ratio between the maximum shear strength $V_{\max }$ and plastic shear strength $V_{p}$, is listed in Table (3.1). Clearly, the values of the overstrength factor are close since the specimens have a very slight difference in the plastic shear strength. Table (3.2) has the maximum values of the shear strength of the tested specimens. The specimens with either bolted or welded braces have an almost equal overstrength factor for both sections. In general, the $\Omega$ factor for T-EBF links is almost the same as that specified by seismic design provision AISC (ANSI/AISC 341-16, 2016) for EBF, which is 1.5 .

Table 3.1 Nominal design properties and section dimensions

\begin{tabular}{|c|c|c|c|c|c|c|c|c|c|}
\hline $\begin{array}{c}\text { Welded } \\
\text { Sections }\end{array}$ & $\begin{array}{c}\mathbf{d} \\
\mathbf{m m} \\
\text { (inch) }\end{array}$ & $\begin{array}{c}\mathbf{t}_{\mathbf{w}} \\
\mathbf{m m} \\
\text { (inch) }\end{array}$ & $\begin{array}{c}\mathbf{b f} \\
\mathbf{m m} \\
\text { (inch) }\end{array}$ & $\begin{array}{c}\mathbf{t}_{\mathbf{f}} \\
\mathbf{m m} \\
\text { (inch) }\end{array}$ & $\begin{array}{c}\mathbf{V}_{\mathbf{p}} \\
\mathbf{k N} \\
\text { (kips) }\end{array}$ & $\begin{array}{c}\mathbf{M}_{\mathbf{p}} \\
\mathbf{k N . m} \\
\text { (kips.ft) }\end{array}$ & $\begin{array}{c}\mathbf{1 . 6} \times \\
\boldsymbol{M}_{\boldsymbol{p}} / \boldsymbol{V}_{\boldsymbol{p}}, \mathbf{m m}\end{array}$ & $\begin{array}{c}\mathbf{e}^{\prime} \\
\mathbf{m m}\end{array}$ & $\mathbf{\Omega}$ \\
(inch) & \\
\hline Tuned_ & 462.28 & 10.236 & 279.4 & 17.272 & 605 & 959 & 2540 & 707 & 1.56 \\
W18x76 & $(18.2)$ & $(0.403)$ & $(11)$ & $(0.68)$ & $(136)$ & $(707)$ & $(100)$ & $(27.832)$ & \\
& & & & & & & & & \\
\hline Tuned_ & 414 & 10.033 & 259.08 & 16.1 & 583 & 775 & 2130 & 692 & 1.52 \\
W16x67 & $(16.3)$ & $(0.395)$ & $(10.2)$ & $(0.634)$ & $(131)$ & $(572)$ & $(84)$ & $(27.2)$ & \\
\hline
\end{tabular}

Table 3.2 Maximum shear strength for all specimens

\begin{tabular}{|c|c|}
\hline Sections & $\begin{array}{c}\text { Maximum shear } \\
\text { strength, kN (kips) }\end{array}$ \\
\hline W16_Welded & $885(199)$ \\
\hline W16_bolted & $894(201)$ \\
\hline W18_bolted & $930(209)$ \\
\hline W18_Welded & $943(212)$ \\
\hline
\end{tabular}




\subsubsection{Fillet Corner Cracks}

Fillet curves are positioned in the web opening corners at the transition zone along the two adjacent edges. Cracks at the fillet curves were observed at low-link rotation due to high-stress concentration during the cyclic test and they propagated in the horizontal direction. Stress concentration resulted in tension at the corners and eventually the cracks. They were spread and grew up with increase of the link rotation. The first observed cracks were at $\gamma=0.02$ radian. However, it is believed the cracks appeared before this rotation but was not observed because the rotation was too small, and the cracks were almost closed cracks. In order to avoid these undesirable cracks, two T-EBF specimens were modified from the original design. The modified specimens were crafted in the same section, W16x67. The first modification was made on the W16x67 welded brace, where the fillet curve radius increased from $0.5^{\prime \prime}$ radius to $3 "$ and the opening edges were changed to $45^{\circ}$. The second modification, on the W16x67 bolted brace specimen was changed by adding high-tensile bolts on the end panels to tighten the flanges and compress the web area in the transition zone during cyclic loading. Figure (3.6) compares crack growth versus the link rotation for three specimens. It shows increased cracking with increasing rotation for both corner sides. The fourth specimen, welded brace W16x67 with a $3 "$ fillet radius and $45^{\circ}$ edge, did not show any cracks at the fillet curve and it performed excellently. Obviously, adding post tension bolts does not prevent cracking. The cracks did not affect link strength except by increasing some end panel rotations, as will be discussed later. 


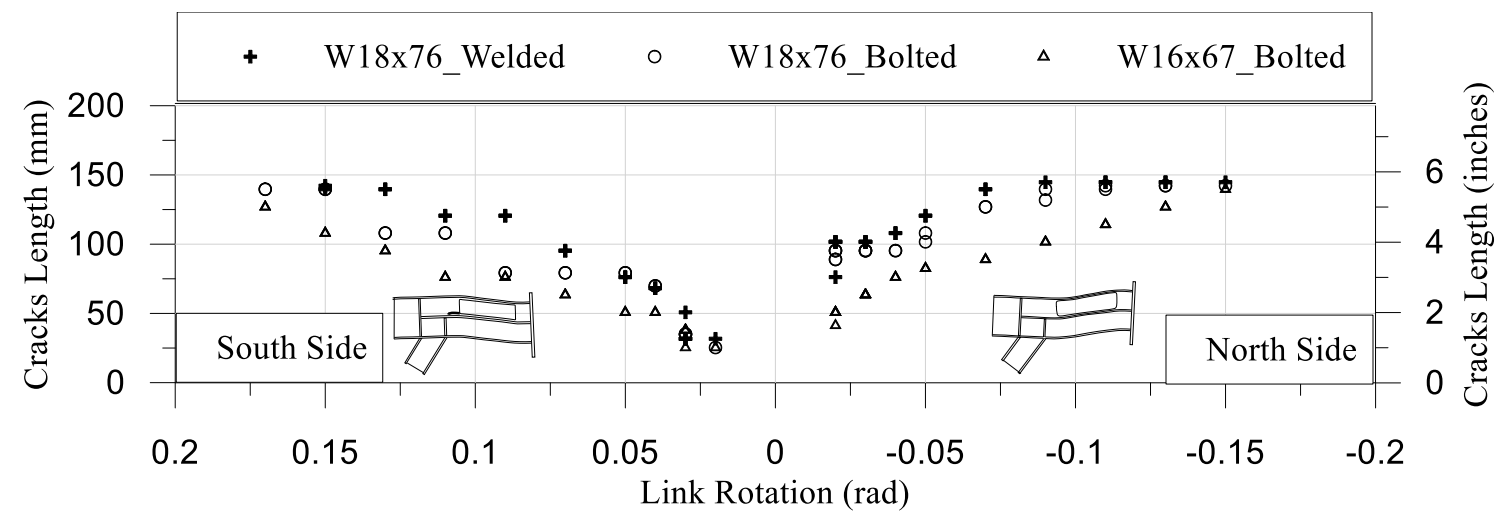

a) Rotation versus measured cracks.
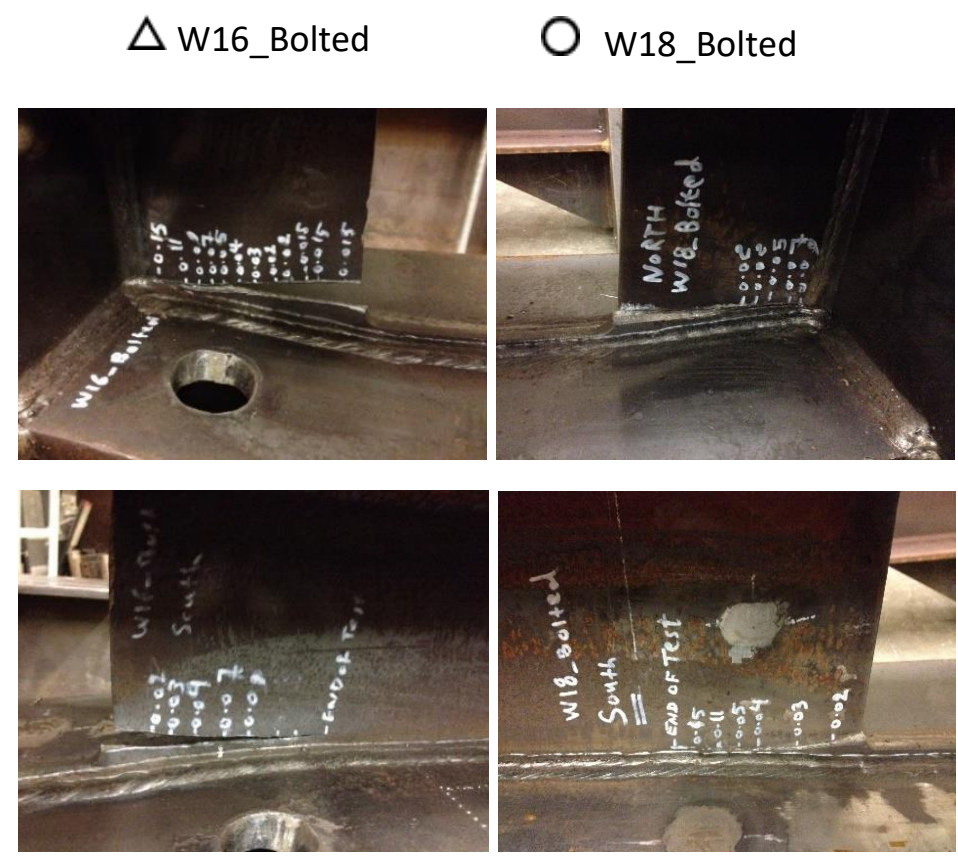

4 W18_Welded
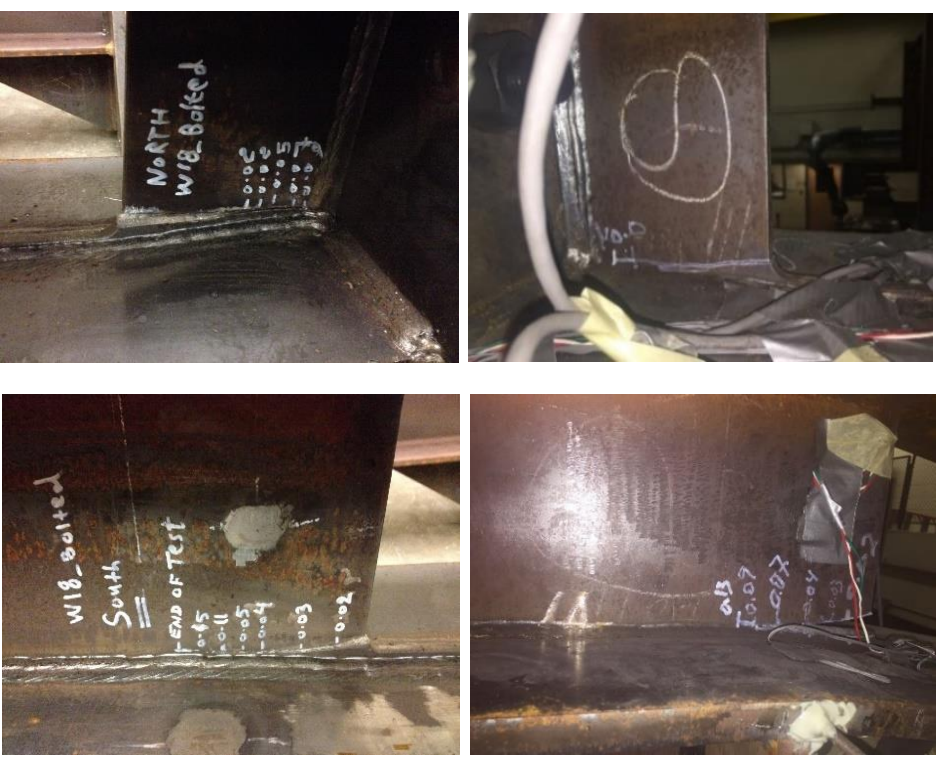

b) Cracks photographs.

Figure 3.6 Opening corner cracks in three specimens at the north and south side 


\subsubsection{Panels' Rotation}

Rotations of six main panels were measured using Linear Variable Differential Transformers (LVDt's) placed diagonally on each panel. Four end panels, one effective panel and one of the opening panels represent the total link. The Equation (2.11) was used to calculate the panel's rotation. Since the link is asymmetrical, the rotational capacity of the panels differs from each other. The back-bone curves of the panels' rotations were graphed with the effective panel for both sections in Figures (3.7) and (3.8). Normalized shear strength was calculated by dividing the tested shear strength on the measured design strength. Clearly, as seen from the panels' rotation of section W16x67, all panel rotations were in the same direction of the effective panel and the value of rotation was smaller compared with the effective panel with the exception of the opening panel, which exhibited almost the same value. The transition zone cracks and plastic flange buckling of section W18x76 have significant and noticeable effects on the rotation capacity of some panels. For instance, the south upper end (CDGH) had a very small rotation value and different rotation direction than the effective panel in positive rotation due to the cracks that appeared in the panel. The south and north lower end panels (ABEF and EFIJ) were also affected by the flange buckling during the negative rotation and they showed small opposite rotation. The north upper panel (GHKL) had very slight rotation in positive rotation due to the hinge formed at the end of the panel near the opening edge. Other panels exhibited the same rotation with the effective panel.

End panels did not display web buckling during the test, and the cracking appeared only in the transient location. Among the all end panels, rotations of the ABEF 
and CDGH panels of section W18 have the maximum values in positive and negative rotation respectively. This is due to cracks that appeared on the transition zone radius, which effect on the rotational resistance capacity. Additionally, from comparing ABEF panel rotations for both sections, cracks have notable effects on increasing the panel rotation. The maximum ratio of $\gamma_{A B E F} / \gamma_{e f f}$. were found 1.173 and 0.27655 for sections W18 and W16 respectively. Panel CDGH also had a high ratio of $\gamma_{C D G H} / \gamma_{\text {eff }}$. for section W18, 0.97, compared with 0.162 for section W16.

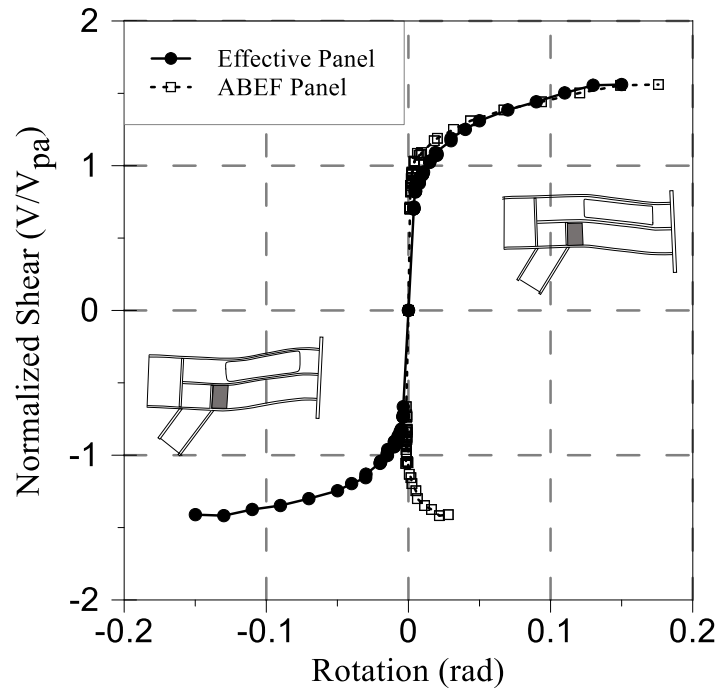

(a)

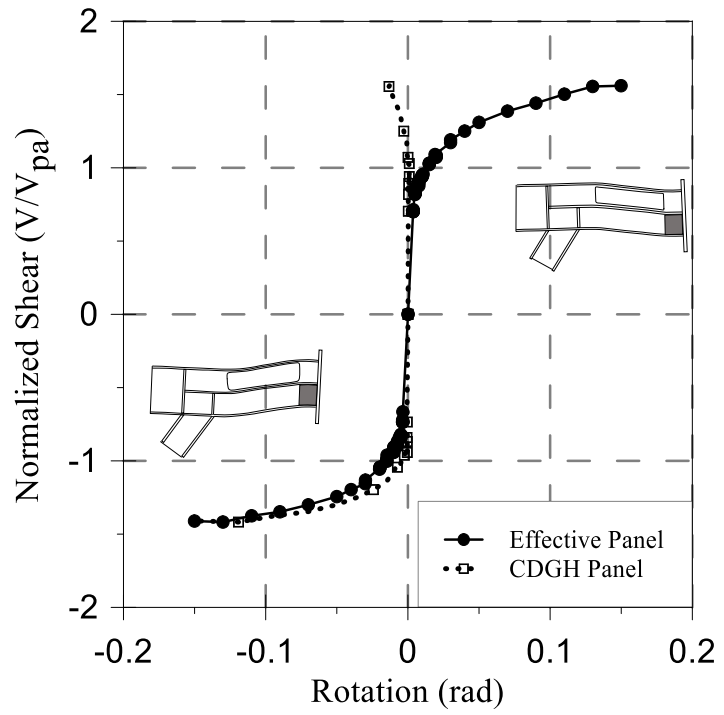

(b) 


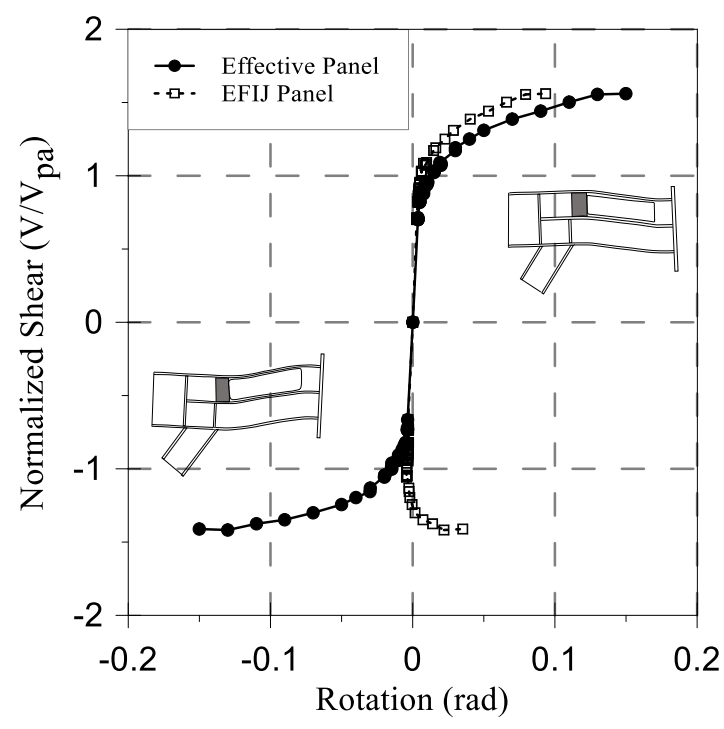

(c)

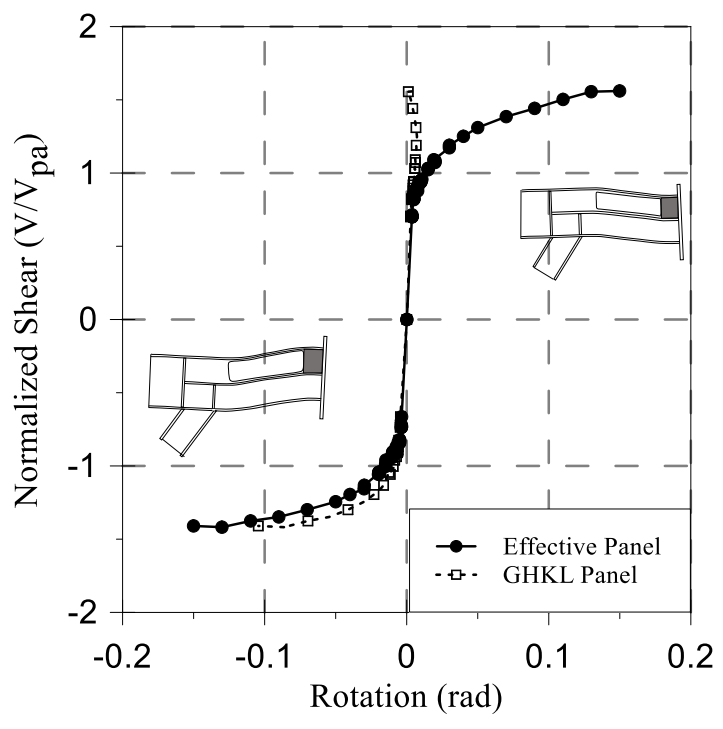

(d)

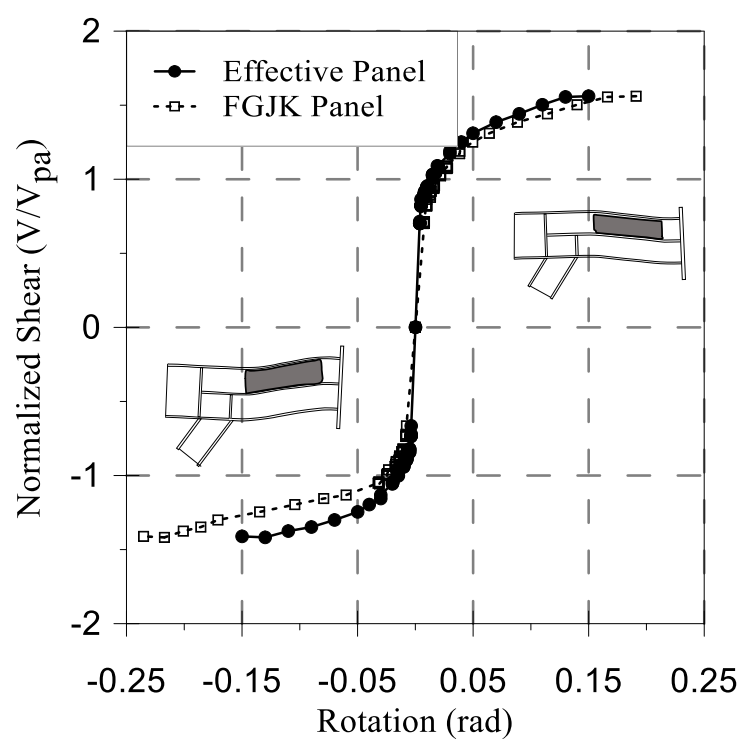

(e)

Figure 3.7 Panels rotation of W18x76 welded section 


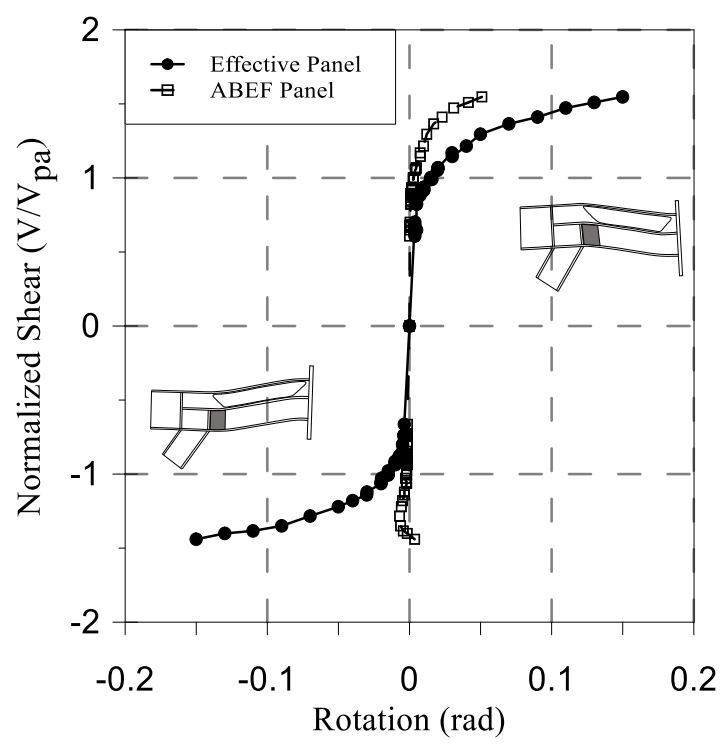

(a)

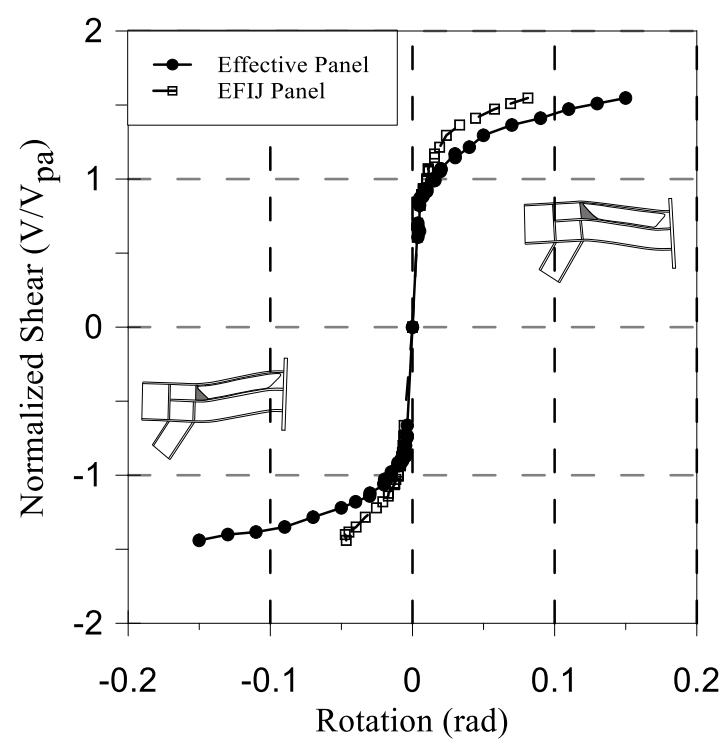

(c)

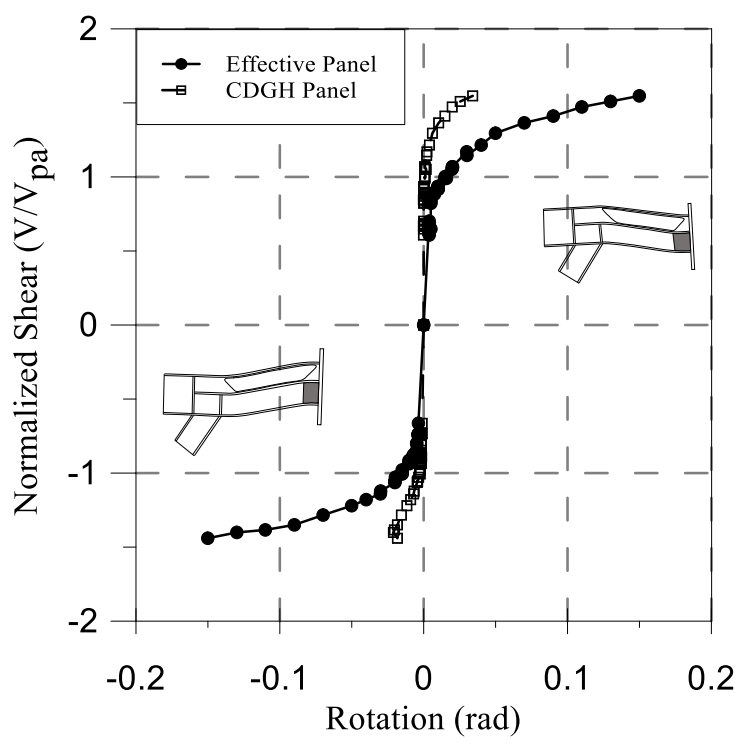

(b)

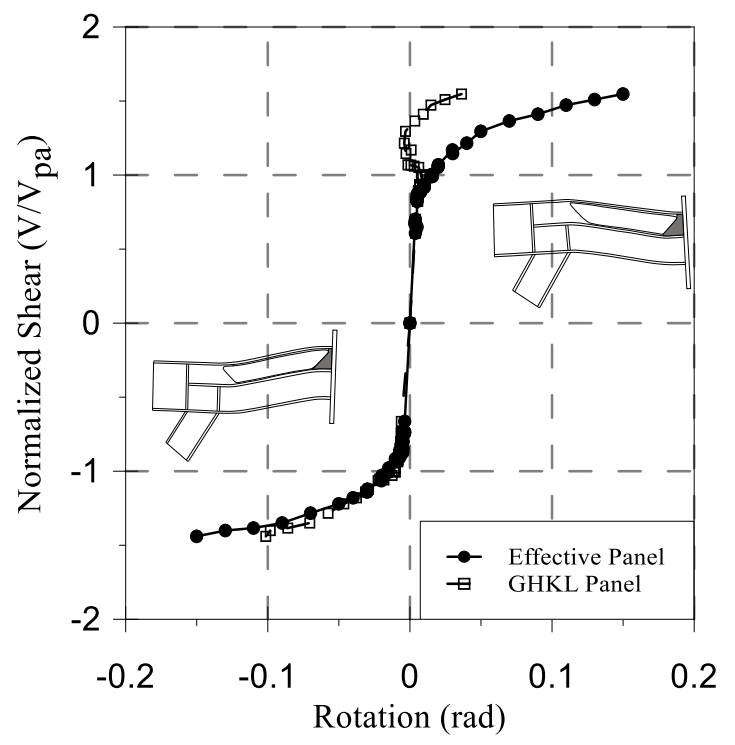

(d) 


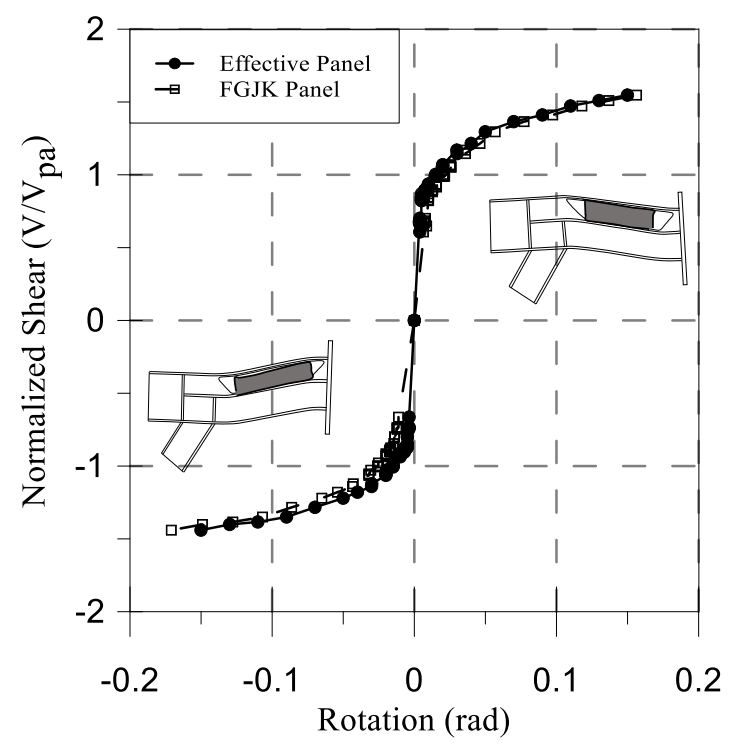

(e)

Figure 3.8 Panels rotation of W16x67 welded section

To quantify the contribution of the effective panel, BCFG, to the total link rotation, the panel contribution completed in one cycle is calculated as follows:

$\left(\Delta_{\text {total }}\right)_{c y c l e}=\left(\frac{\gamma_{1} \times e_{1}+\gamma_{2} \times e_{2}+\gamma_{3} \times e_{3}}{e_{1}+e_{2}+e_{3}}\right)_{c y c l e}$

Where; $\Delta_{\text {total }}=$ Vertical movement by the total link rotation, $\gamma_{i}=$ Calculated panel shear deformation, and $\mathrm{e}_{\mathrm{i}}=$ Length of the panel

By substituting the panels' name shown in Figure (3.9), the equation 3.1 becomes;

$\left(\Delta_{\text {total }}\right)_{c y c l e}=\left(\frac{\gamma_{(A B E F)} \times E D+\gamma_{(B C F G)} \times e^{\prime}+\gamma_{(C D G H)} \times E D}{E D+e^{\prime}+E D}\right)_{c y c l e}$

Where; $\mathrm{e}^{\prime}=$ Effective panel length, and $\mathrm{ED}=$ End distance $=$ Length of the end panels 


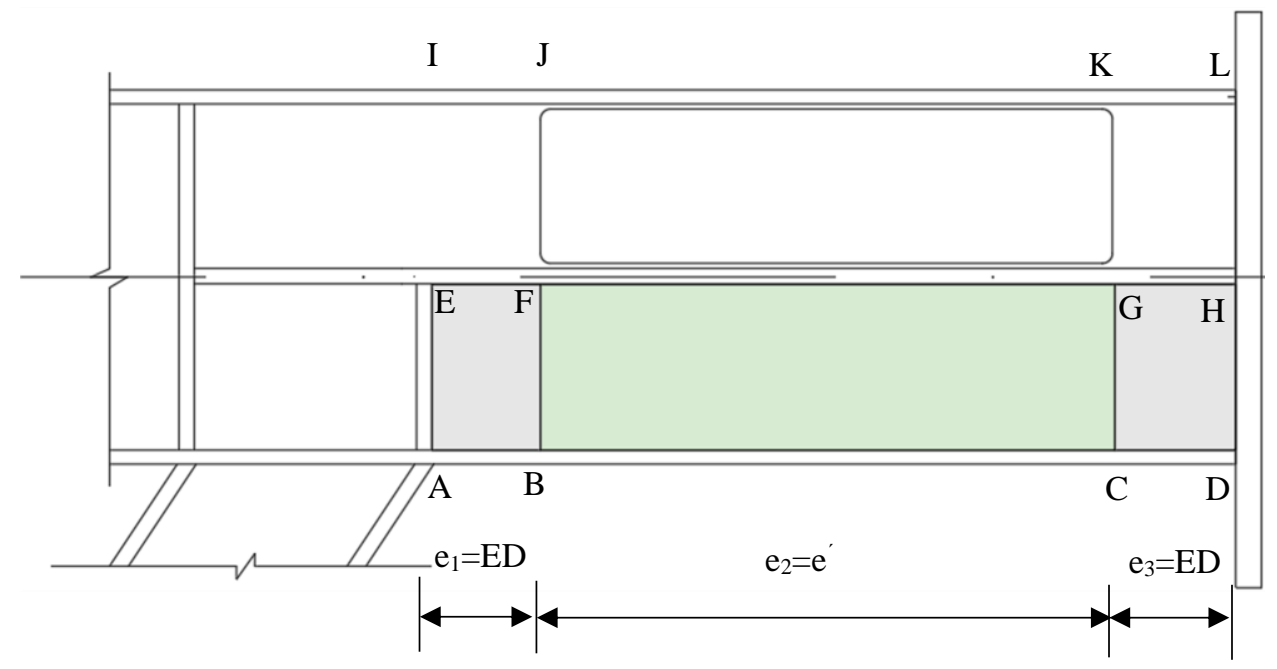

Figure 3.9 Panels' name within the link

The total rotation can be calculated using equation (3.3);

$\left(\gamma_{\text {total }}\right)_{c y c l e}=\left(\frac{\Delta_{\text {total }}}{e}\right)_{c y c l e}$

In which $\mathrm{e}=$ Total length of the link $=e^{\prime}+2 \times E D$

Therefore, the effective panel contribution percentage,Cont. \%, is equal to:

(Cont. $\%)_{\mathrm{BCFG}}=\frac{\Delta_{B C F G}}{\Delta_{\text {total }}} \times 100$

Tables (3.3) and (3.4) present the experimental vertical movements translated from the panels' rotations, $\Delta=\gamma \times \mathrm{e}$, for specimens W16_welded and W18_welded respectively. The vertical displacements shown in the tables correspond to effective panels' rotations $0.09 \mathrm{rad}$. The contribution percentages of the effective panel, which are 
computed up to a rotation equal to $0.15 \mathrm{rad}$, is shown in Figure (3.10). It was found that the contribution of the effective panel's rotation to the total link rotation was on average about $94 \%$ for the specimen without transition zone cracks and $88 \%$ for the specimen with the cracks.

Table 3.3 Displacement deformations of three panels translated from the panels' rotations (specimen W16_Welded)

\begin{tabular}{|c|c|c|c|c|}
\hline $\begin{array}{l}\text { Panel's } \\
\text { name }\end{array}$ & $\gamma(\mathrm{rad})$ & $\begin{array}{l}\text { Panel's length } \\
\text { (in) }\end{array}$ & $\begin{array}{c}\Delta \\
\text { (in) }\end{array}$ & $\gamma_{\text {total }}$ \\
\hline ABEF & 0.023 & 6 & 0.14 & \multirow{3}{*}{0.0677} \\
\hline $\mathrm{BCFG}$ & 0.09 & 27.25 & 2.45 & \\
\hline $\mathrm{CDGH}$ & 0.014 & 6 & 0.08 & \\
\hline $\mathrm{ABEF}$ & -0.006 & 6 & 0.009 & \multirow{3}{*}{-0.0652} \\
\hline $\mathrm{BCFG}$ & -0.09 & 27.25 & 2.45 & \\
\hline $\mathrm{CDGH}$ & -0.018 & 6 & 0.1 & \\
\hline
\end{tabular}

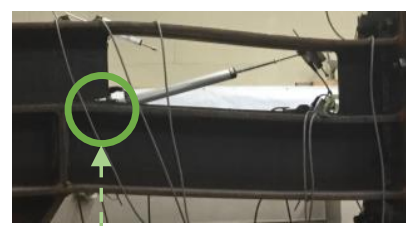

Table 3.4 Displacement deformations of three panels (specimens W18_Welded)

\begin{tabular}{|c|c|c|c|c|}
\hline $\begin{array}{c}\text { Panel's } \\
\text { name }\end{array}$ & $\gamma(\mathrm{rad})$ & Panel's length (in) & $\begin{array}{c}\Delta \\
\text { (in) }\end{array}$ & $\gamma_{\text {total }}$ \\
\hline $\mathrm{ABEF}$ & 0.094 & 6 & 0.564 & \multirow{3}{*}{0.075} \\
\hline BCFG & 0.09 & 27.8 & 2.5 & \\
\hline $\mathrm{CDGH}$ & -0.0087 & 6 & -0.052 & \\
\hline $\mathrm{ABEF}$ & 0.011 & 6 & 0.066 & \multirow{3}{*}{-0.071} \\
\hline $\mathrm{BCFG}$ & -0.09 & 27.8 & -2.5 & \\
\hline $\mathrm{CDGH}$ & -0.07 & 6 & -0.42 & \\
\hline
\end{tabular}

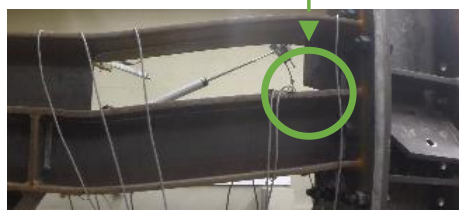




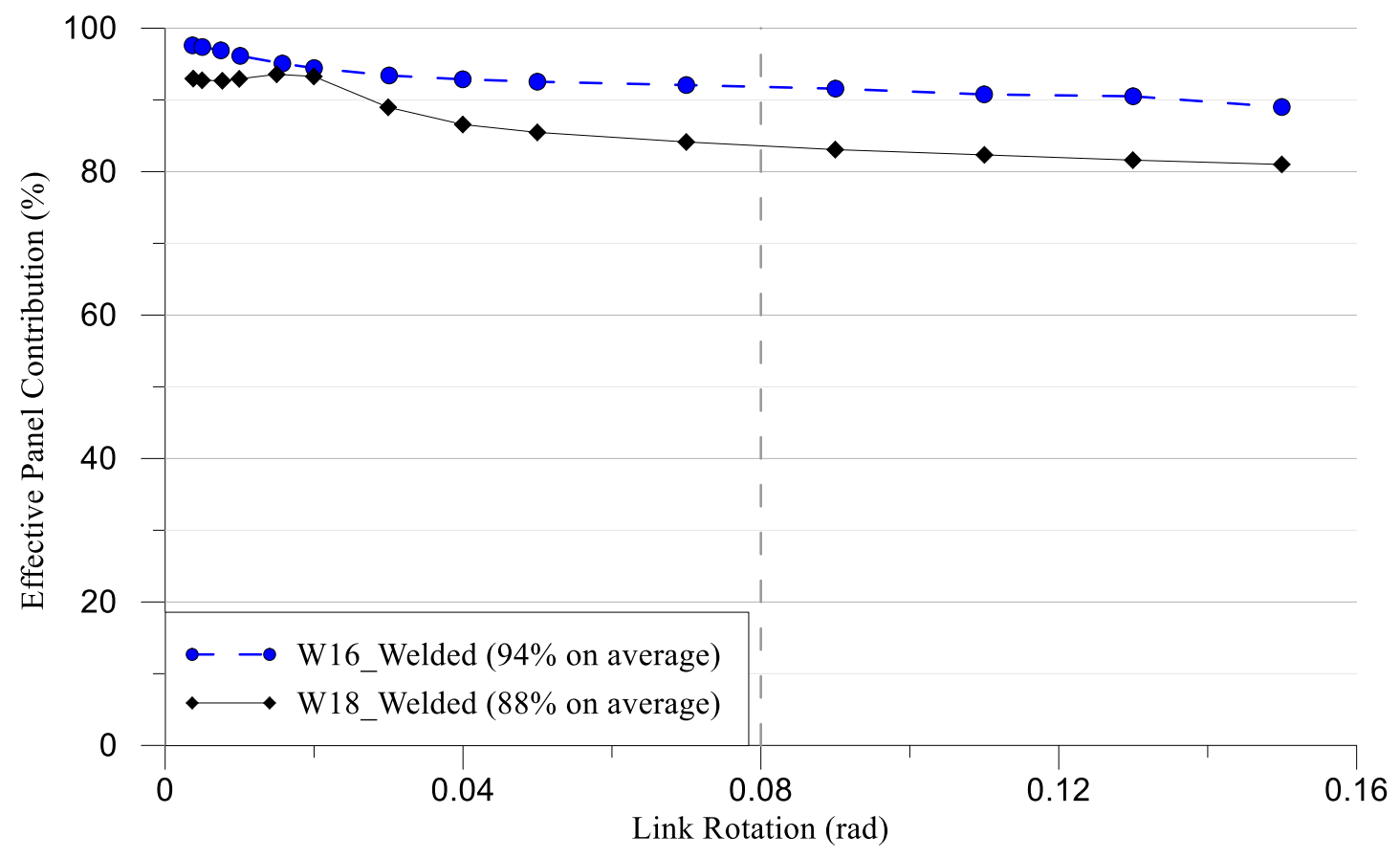

Figure 3.10 Contribution of the effective panel rotation to the total link rotation

\subsubsection{Shear Strain Discuss (Welded Brace)}

Five rosette strain gauges were attached on the link web: three of them were placed on the effective length panel at mid height, and two rosette gauges were glued on the mid depth of the ABEF and EFIJ panels. The link is asymmetrical, and it has two different rotational resistances. The asymmetrical link in EBF leads to generation of an axial force in the link. The lateral stiffness of the shear link comes from the shear strength and specifically from shear strain hardening during the yielding of the link (Yang, 1982). Shear strains were distributed non-uniformly along the measured area and were not the same, which means that shear strain at negative rotation differs from shear strain at positive rotation. Additionally, the shear strain is different over the measured distance. 
Clearly, as demonstrated in the relationship between the rotation and shear strain shown in Figure (3.11), the maximum shear strain of the link when the rotation is negative, is larger than when the rotation is positive. The bracing member plays a significant role in this behavior since it is in tension.

\subsubsection{Strain Development (Welded Brace)}

Negative and positive strains refer to compression and tension respectively. Strain gauges used in this study were placed on the most critical location at the opening edge near the bracing member side where high shear and moment are expected. Uniaxial strain gauges were attached on the outer fibers of the top and bottom flanges and on top of the middle flange to investigate the strains along the flanges at the locations. The graphs in Figure (3.12) and (3.13) demonstrate that when the rotation is negative, for both tested sections, the tensile strain was the main strain for the bottom flanges and it increased with an increase in the rotation, especially in the area near the bracing member due to an increase in the sloping of the flange bent. In contrast, the strains of the top and middle flanges measured compressive strain since the flanges bent clockwise at these locations and strain gauges were placed on the upper surfaces. In the opposite rotation (positive rotation), the strains of the W16 section occurred as compressive strains in the bottom flanges and tensile strains in the middle and top flanges. This is not the case in W18x76, when it is clearly depicted in the graphs that the bottom flange strains changed from compressive to tensile strain or produced a decreasing of the strain with an increase in rotation. The reason for this change is that a local plastic buckling of the bottom flange near the bracing member formed, causing a plastic sectional deformation. The middle 
flange also formed a plastic local buckling and, therefore, the area near the bracing, ABEF panel, was in tension and gradually increased while strain on the further area became lower approaching near zero. Maximum measured strain was calibrated to be $0.0095 \mathrm{in} /$ in so up-to-rotation equal to 0.07 radian can be captured and discussed.
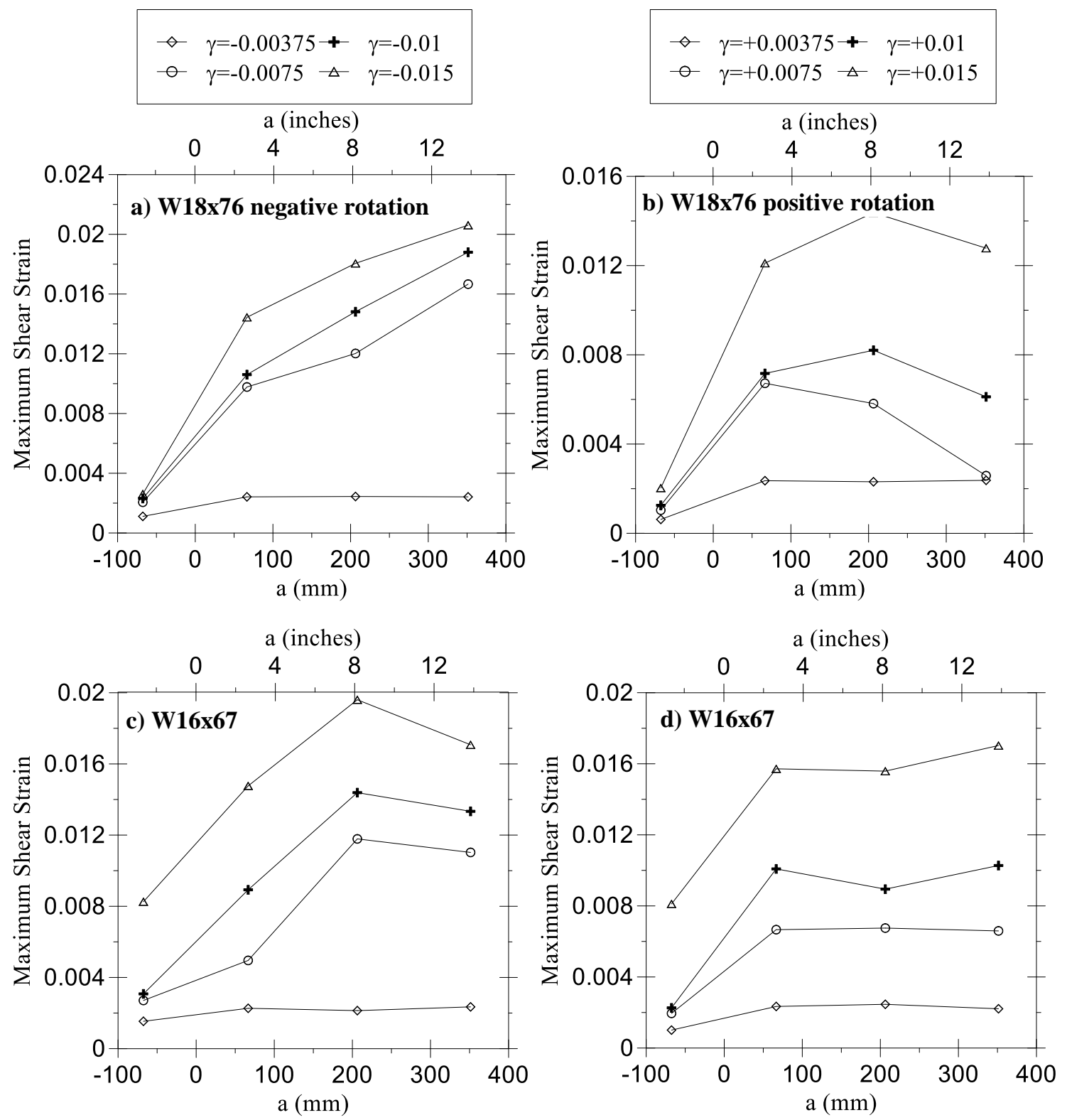

Figure 3.11 Maximum shear strain at the webs: 

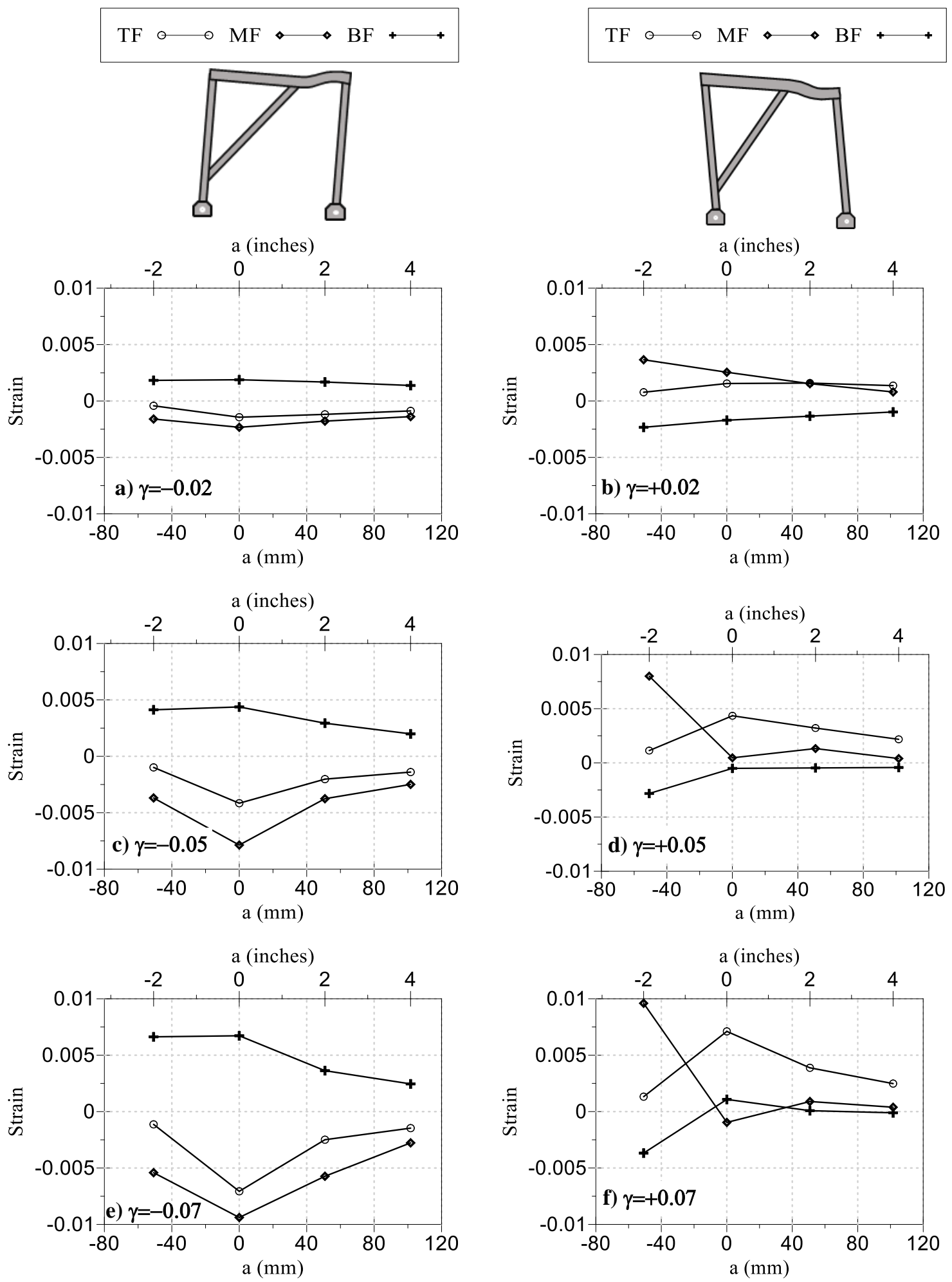

Figure 3.12 Strain at the flanges of W18 welded brace 


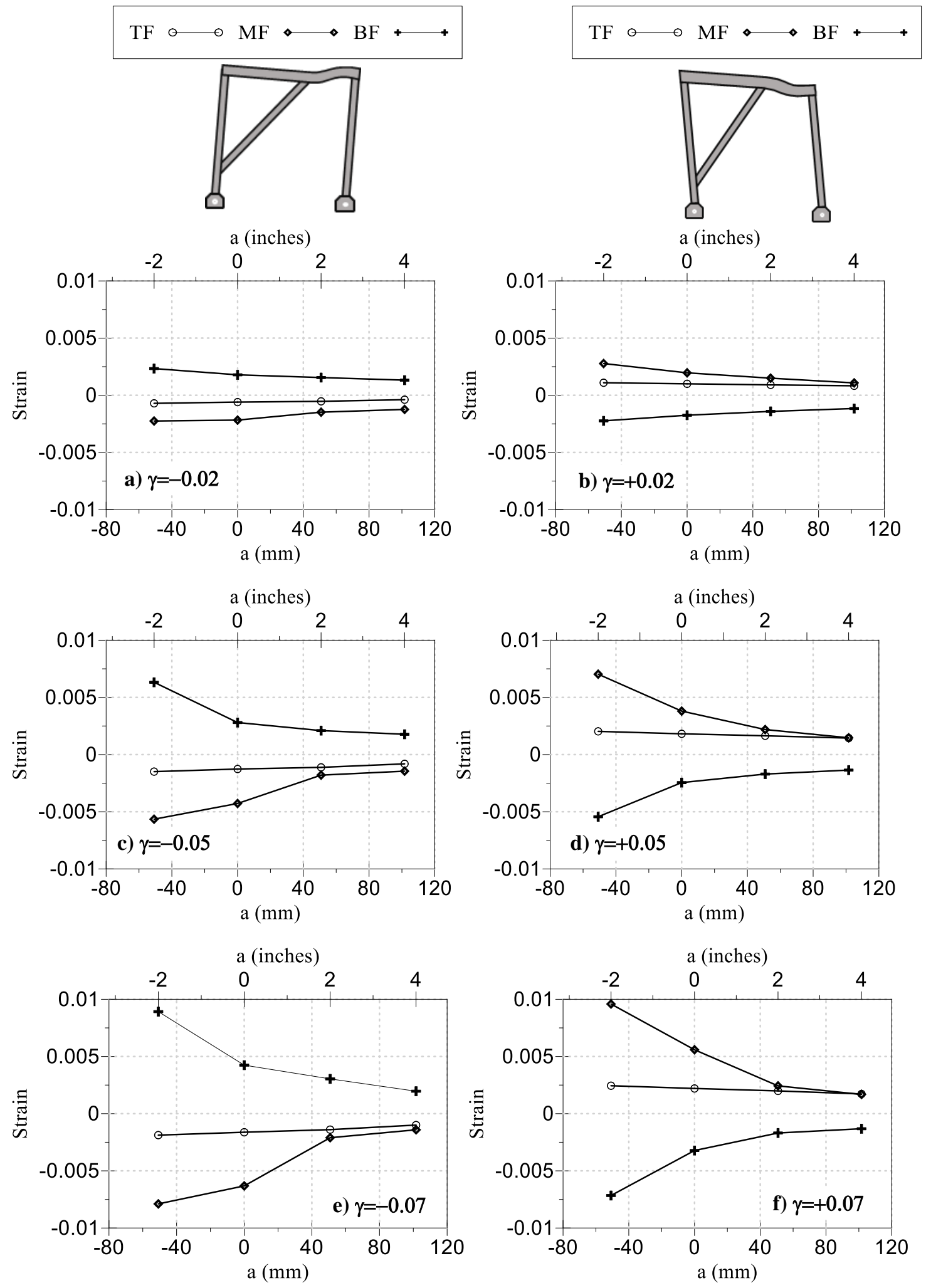

Figure 3.13 Strain at the flanges of W16 welded brace 
In addition to the panel's rotation hysteretic response, and strain analysis comparison between brace welded and bolted was also discussed as it is difficult to assess the suggested tuned-EBF for the bolted braced-link connection. All four full-scale TEBFs passed the seismic design requirements and the discussion here will help to understand the effectiveness of the new brace-link connection on the panels and all links.

\subsection{Compare strains for both welded and bolted brace}

\subsubsection{Shear Strain}

The typical strain gauge locations are shown in Figure (2.21). In general, the shear strains at the middle of the link are higher compared with the link ends for the same rotation value. Comparison was made between shear strains resulting from the web links for both welded and bolted brace-link connections. Figure (3.14) shows the maximum shear strain versus the link rotation of the two end panels, ABEF and EFIJ, while Figure (3.15) describes the shear strain distribution along the link. The slight differences between the behaviors of T-EBF for the two connection types were evident on the influence of the thick plate used in the bolted-brace link connection on the rotational stiffness of the effective and end panels. The smaller value of the maximum shear strain versus negative link rotation at the center of the ABEF and EFIJ for the bolted-brace link connection may indicate that the brace connection in the bolted-brace link connection is not fully rigid, especially when the brace connection bolts come under tension.

Therefore, the rotational stiffness of the bolted-brace link connection is less than that of the case when the brace is welded with the link. In contrast, when the link rotation is positive, inelastic shear deformation increases as the thick plate of the bolted-brace link 
connection applies more compression to the panels. The figure also shows that the shear strain at the center of the link of the bolted connection becomes close the link rotation. The inelastic shear deformation increased in the center of the links, which is intended by design to concentrate the inelastic deformation within the web effective panel of the link.

\subsubsection{Flange Strains}

Figure (3.16) shows the relation between the rotation and the longitudinal strain in the link middle flanges for both types of T-EBF brace connection, W18x76 welded and W18x76 bolted. The plotted strain represents the strain gauge located at the edge of the web opening, as indicated in the stain pattern shown in Figure (2.21). Clearly, the strain increased gradually with increase in the link rotation. It appears that the two sections have different sign patterns in positive rotation due to local flange buckling at this location in the welded brace connection configuration. When the local buckling deforms, the local stress sign changes due to transverse the slope of the bending curvature. In contrast, strain sign was the same during the negative rotation and there were no local flange buckling effect on the slope curvature. 

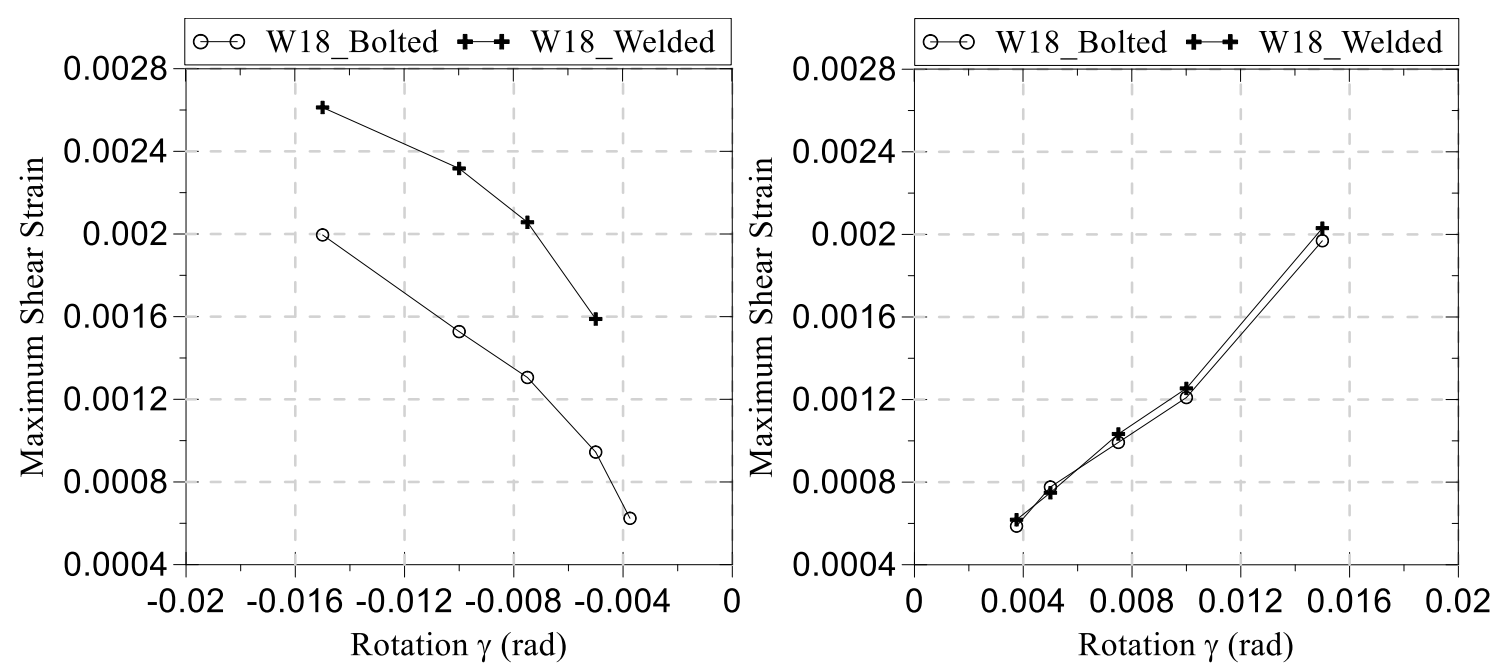

a) $\mathrm{ABEF}$ end panel
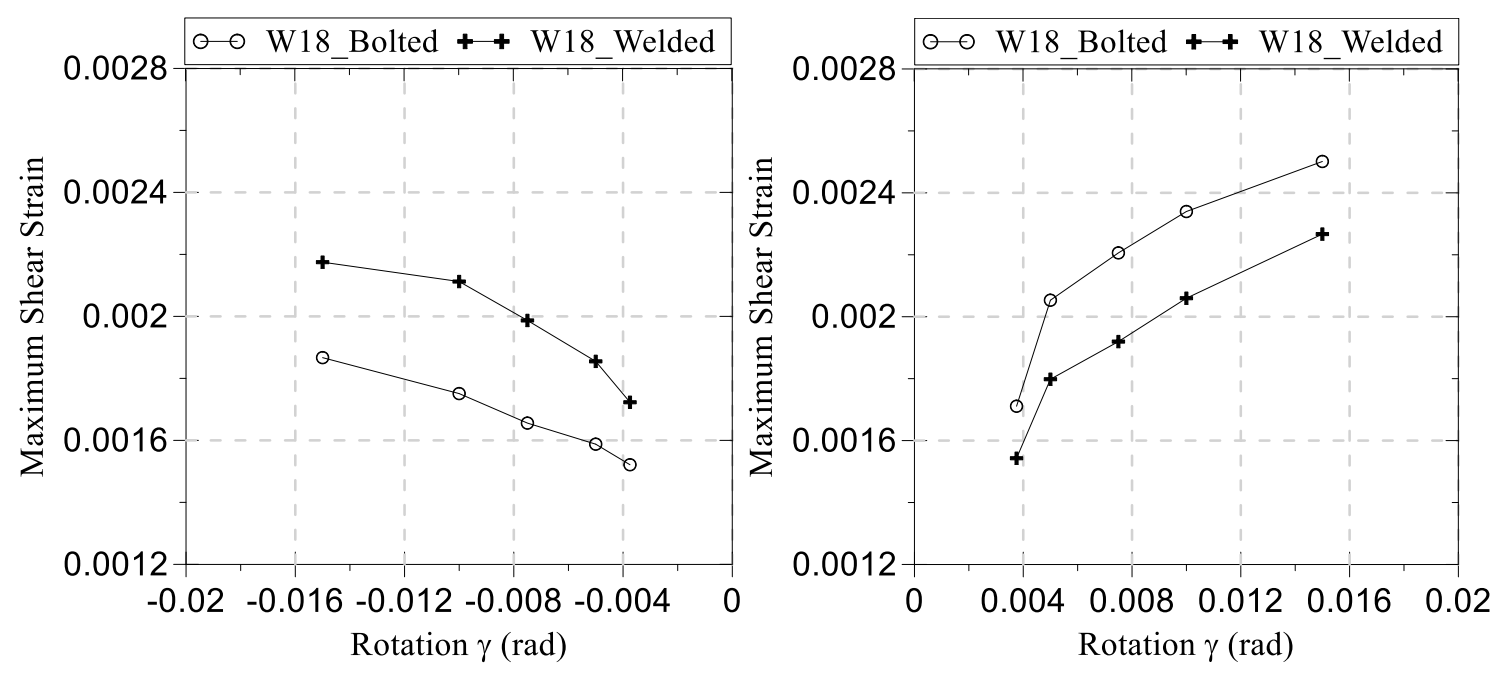

b) EFIJ end panel

Figure 3.14 Web shear strain 

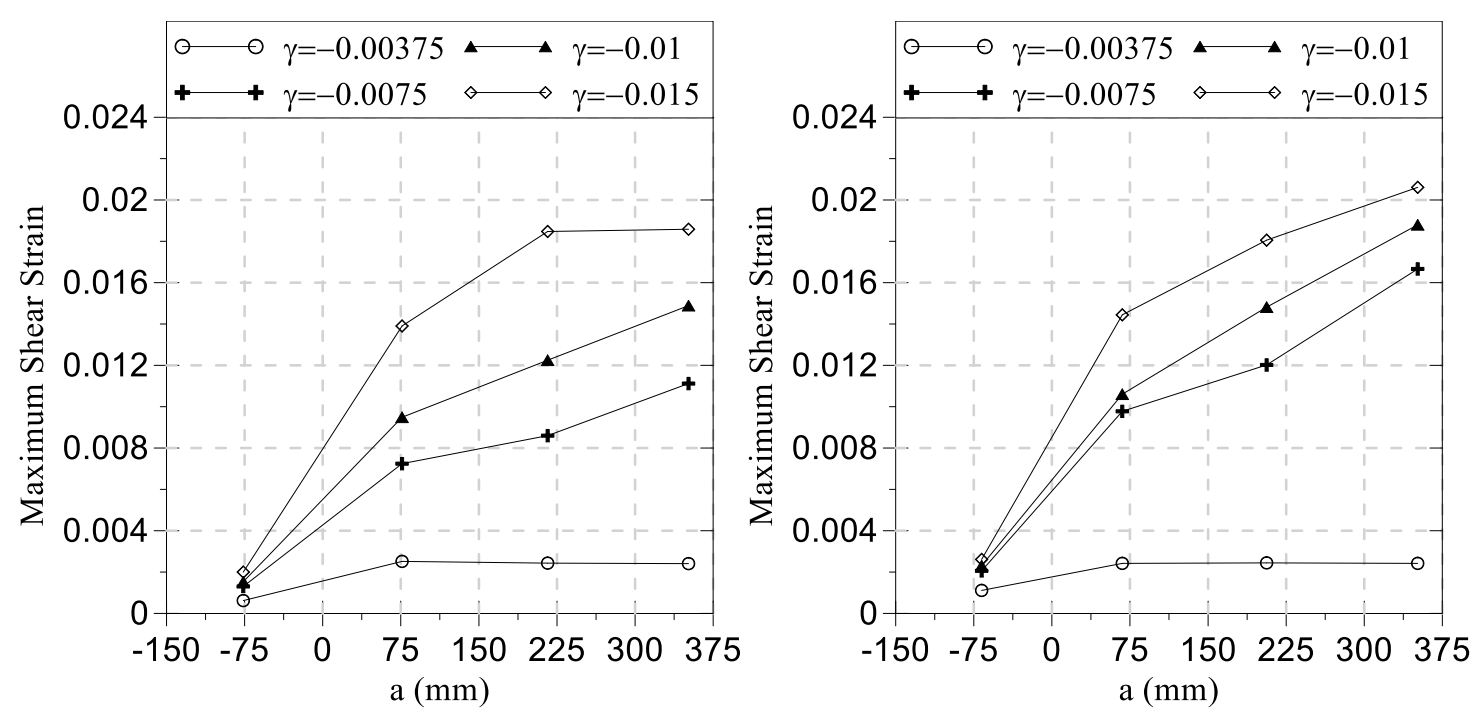

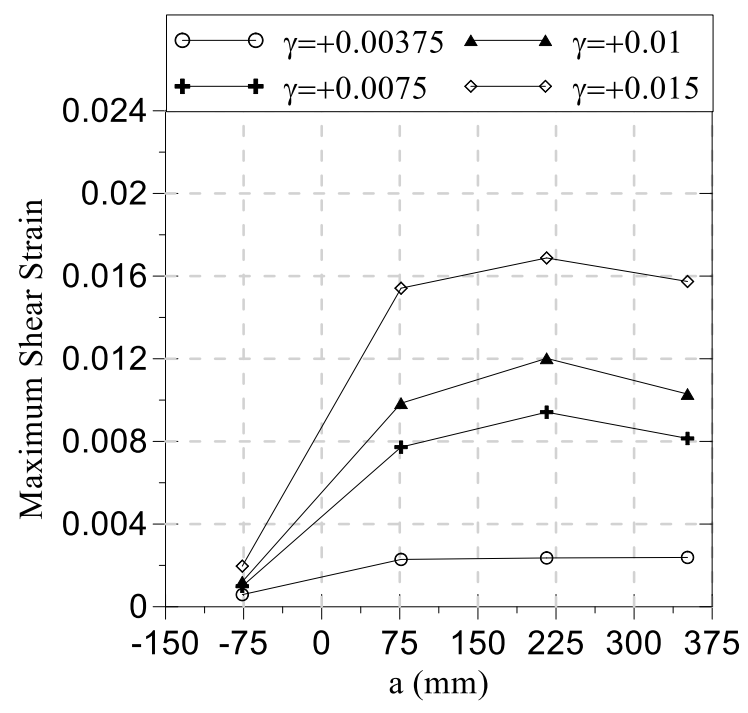

(a) W18x76 Bolted

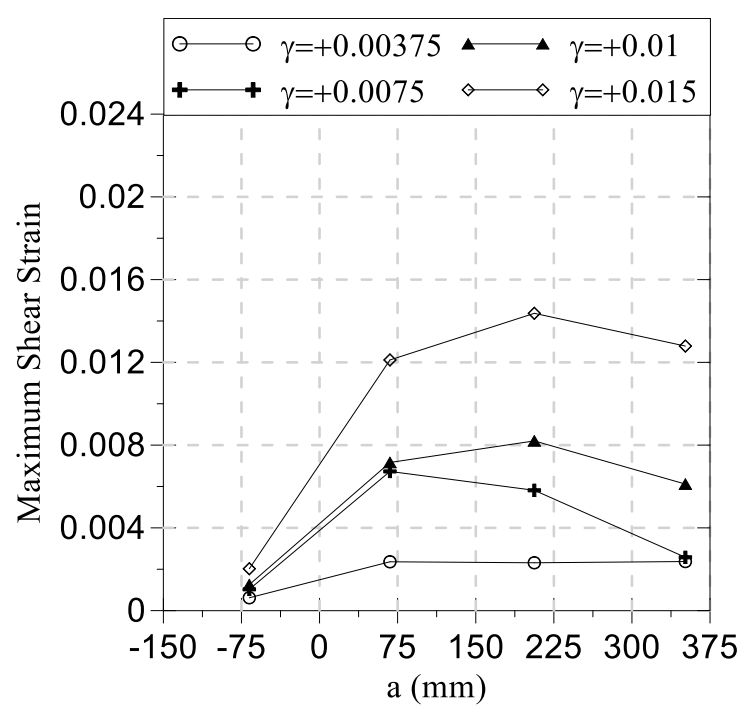

(b) W18x76 Welded

Figure 3.15 Web shear strain distribution 



Figure 3.16 Middle flange strain distribution at the opening edge 


\section{Chapter 4: Numerical Modeling}

\subsection{Introduction}

In this chapter, the finite element analysis is presented to simulate and validate the experiment presented in Chapter 2. The primary purpose of the simulation is to extend and generalize these findings. The FEA models were created using package program Abaqus/Standard. The results from FEA helped to obtain more detail about local stresses and strains at the region of interest. Even though fractures were not modeled, the developed stresses and plastic strain distributions allowed for predictions as to expected fractures. Comparing results from FEA and experimental works has shown that FEA can capture local buckling.

\subsubsection{General Notes of the Model}

1) Since the main objective of this work is to predict the performance of the T-EBF, focusing on the behavior of the shear link, welds and bolts were not modeled so as to reduce computational complexity and limit the number of elements and running time of the model.

2) For the same reasons, the columns and parts of the beam and bracing member were modeled using wire as an element type with elastic material properties.

3) Displacement control was applied to capture the link rotation. 


\subsection{Model Definition}

The experimental assembly setup was modeled and evaluated to imitate hysteresis behavior. The inelastic material properties suggested by Volynkin (2016) were inputted in the analysis of the link, stiffeners, and end plate as a combined isotropic and kinematic hardening model, which is the most sophisticated model for cyclic loading. Some components were not assigned plastic properties, since they remained in the elastic stage. For this reason, isotropic hardening was used for the columns, the beam out of the link, and part of the bracing member.

A convergence mesh study was carried out in terms of stresses and plastic strains. The finite element analysis was verified against the experimental work for both sections. The rotation-shear curves were graphed to show this comparison. The finite element simulations were used to generate new findings and to evaluate the proposal T-EBF.

\subsubsection{Material Property}

As described in Chapter 2, the T-EBF were made from different steel grades. Therefore, three sets of material properties were assigned for the FE of the model. The cyclic hardening and Bauschinger effect were considered in the shear link model, since it is the part expected to dissipate the seismic energy through the inelastic shear strain, possibly buckling its web during the inelastic deformation. Table (4.1) contains the elastic, nonlinear kinematic, and isotropic parameters for the material properties used in the analysis. Brief definitions of the model parameters, combined isotropic and kinematic 
hardening model are available in the Appendix B, however full explanation thereof is beyond the scope of this research.

Table 4.1 Combined Isotropic\& Kinematic material parameters used in FEA Volynkin (2016)

\begin{tabular}{|c|c|c|c|c|c|c|c|c|c|c|}
\hline $\begin{array}{l}\text { Steel } \\
\text { Grade }\end{array}$ & $\begin{array}{c}\mathbf{E} \\
\text { Mpa } \\
(\mathbf{k s i})\end{array}$ & $\begin{array}{c}\sigma_{\mathbf{y}} \\
\text { Mpa } \\
(\mathbf{k s i})\end{array}$ & $\begin{array}{c}\mathrm{C}_{1} \\
\mathrm{Mpa} \\
(\mathbf{k s i})\end{array}$ & $\gamma_{1}$ & $\begin{array}{c}\mathrm{C}_{2} \\
\mathrm{Mpa} \\
(\mathbf{k s i})\end{array}$ & $J^{2}$ & $\begin{array}{c}\mathrm{C}_{3} \\
\mathrm{Mpa} \\
(\mathrm{ksi})\end{array}$ & $y^{3}$ & $\begin{array}{c}\mathbf{Q}_{\infty} \\
\text { Mpa } \\
(\mathbf{k s i})\end{array}$ & b \\
\hline A992 & $\begin{array}{l}213600 \\
(30980)\end{array}$ & $\begin{array}{l}152.23 \\
(22.08)\end{array}$ & $\begin{array}{l}18616 \\
(2700)\end{array}$ & 156 & $\begin{array}{l}101711 \\
(14752)\end{array}$ & 650 & $\begin{array}{l}408.2 \\
(59.2)\end{array}$ & 0 & $\begin{array}{c}286.4 \\
(41.54)\end{array}$ & 0.29 \\
\hline A572 & $\begin{array}{l}199948 \\
(29000)\end{array}$ & $\begin{array}{c}379.2 \\
(55.00)\end{array}$ & $\begin{array}{c}10755.8 \\
(1560)\end{array}$ & 75.0 & - & - & - & - & - & - \\
\hline Elastic & $\begin{array}{l}199948 \\
(29000)\end{array}$ & - & - & - & - & - & - & - & - & - \\
\hline
\end{tabular}

\subsubsection{Boundary Conditions}

The boundary condition for the model is illustrated in Figure (4.1). The wire column was fixed at the base in the all three displacement directions, $\mathrm{Ux}=\mathrm{Uy}=\mathrm{Uz}=0$, but with allowing rotation about the global axis $\mathrm{X}, \mathrm{Y}$, and $\mathrm{Z}$ to simulate a pinned end. Moreover, the frame was prevented from moving in $\mathrm{X}$ direction, the perpendicular direction, in the two upper points that connected the beam with the column, to maintain the movement in the Z-Y plane and to prevent out of plane movement. Displacement control was employed to apply lateral displacement at the location of the actuator centerline. 


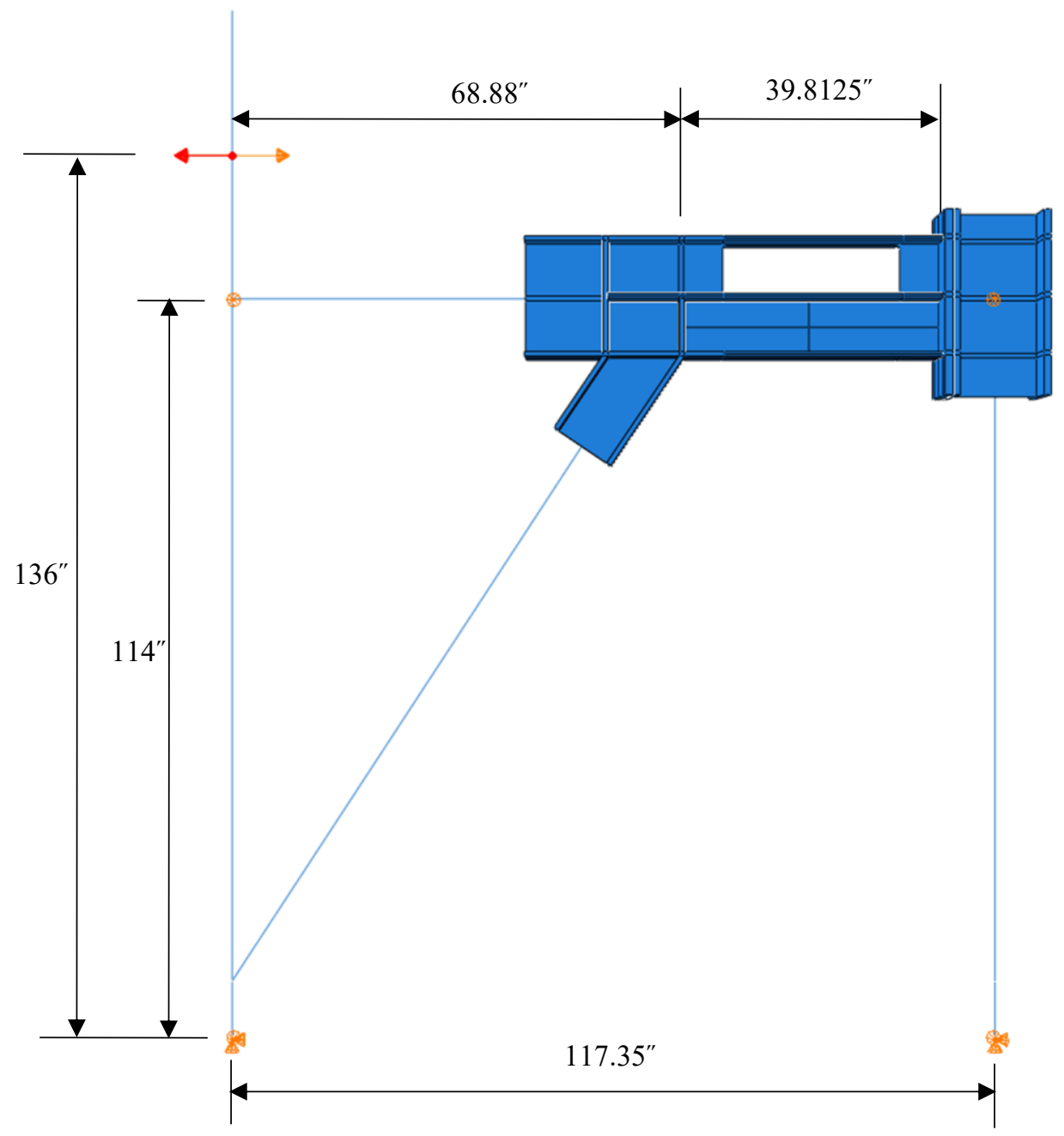

Figure 4.1 F.E.A model

\subsubsection{Loading Protocol}

The frame was connected with the actuator as shown in Figure (4.1). The lateral load was applied by a pushing/pulling the frame through applying displacement in the ZY plane. The applied displacements were chosen to achieve the rotational loading protocol in the link. The displacements were recorded after calculating the link rotation at 
each step and compared with the loading protocol. Then, the verified z-displacements were defined as boundary conditions in the Z-direction.

\subsubsection{Model Parts}

The frame model contained many individual parts to generate the whole structure. The model parts included two columns of section W14x145, which had the section properties profile, a bracing member of $\mathrm{W} 10 \times 67$, beams of either section $\mathrm{W} 18 \times 76$ or W16x67, end plates of 1.25 " thickness, horizontal stiffeners and vertical stiffeners $3 / 4$ " in thickness.

\subsubsection{Partitions and Contact Surfaces}

To combine the parts into the assembly, contact surfaces needed to be defined. Therefore, after the parts were created and partitioned, the slave and master surfaces were carefully chosen and determined. Then, they were connected. The stiffeners were bonded with the beam using tie constraints, and the beam attached, with the end plate at the flanges and web surfaces, by the same type of tie constraints. Kinematic coupling was used to connect the wire with the $3 \mathrm{D}$ solid at the end plate-column wire connection.

\subsection{Element Types and Mesh}

Eight node linear hexahedral brick elements (C3D8) with first order shape function were selected to represent the link, endplate, stiffeners, and bracing member. Three-dimensional full integration element was used to control the meshing. C3D8 was better to model shear links than shell elements due to its ability to capture post-buckling performance under large inelastic rotation (Stephens and Dusicka, 2011). Also, shell 
elements showed development flange buckling at lower link rotation compared with the 3D continuum. The columns, bracing member, and a part of the beam were modeled using wire planar. A two-node linear beam in space, which functions as a shear-flexible element, was represented in the W14x145 columns, W18x76 and W16x67 collector beams, and part of the W10x68 bracing member, since they performed elastically. Because the link was expected to achieve large inelastic rotation and deformation, the geometry nonlinearity was activated.

\subsection{Convergence Study}

The mesh refinement studies of the model were conducted to select the level of mesh refinement necessary to achieve the objectives. Figure (4.2) represents the typical mesh refinement used throughout the research. The mesh convergence study had various element sizes of $0.8,0.6,0.489$, and 0.445 for cyclic analysis, and more sizes for monotonic analysis. The study included the specimens described in Chapter 2: W18x76 Welded, W18x76 Bolted, and W16x67 Welded.
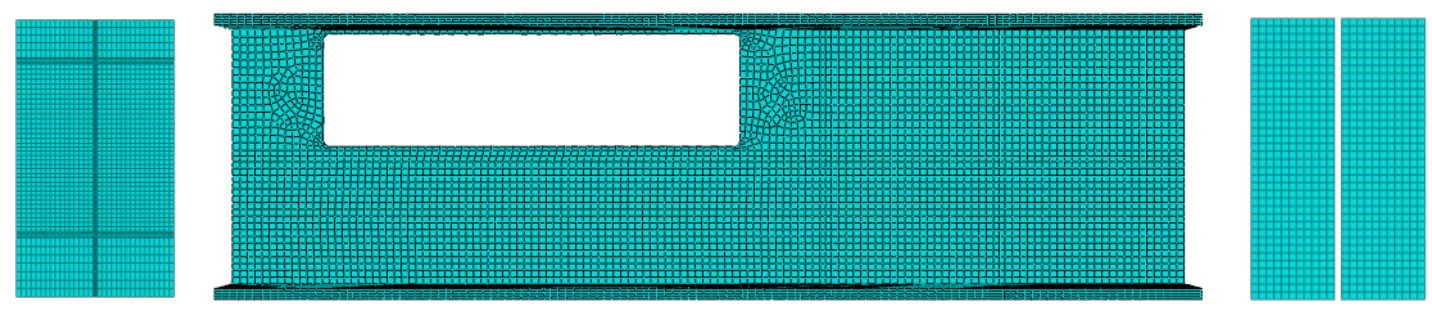
a) End plate
b) Beam and link
c) Vertical stiffeners 


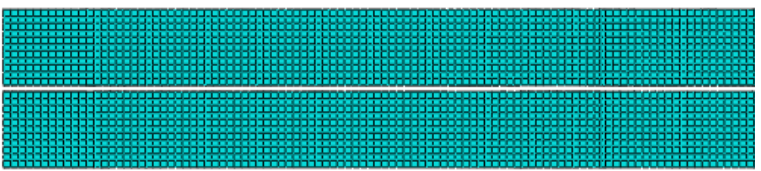

d) Horizontal stiffeners

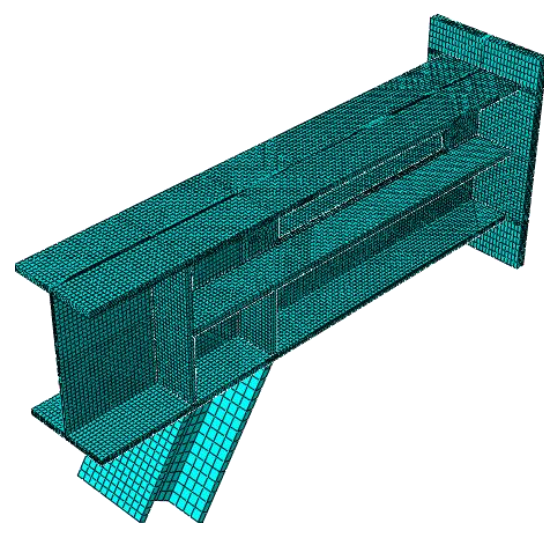

e) Assembly parts

Figure 4.2 Typical model mesh

Convergence was defined as equivalent plastic strain and von Mises versus number of beam-link elements. It was checked at the most affected point, which was the center of the effective panel. It was also checked at the middle of the top flange at the end of the link, close to the endplate. The final mesh in the convergence required a total number of elements for the three FEM models ranging from 37,000 to 60,000. The results for strains and stresses at the interested points at different link rotation for both monotonic and cyclic loads are shown in Figure (4.3).

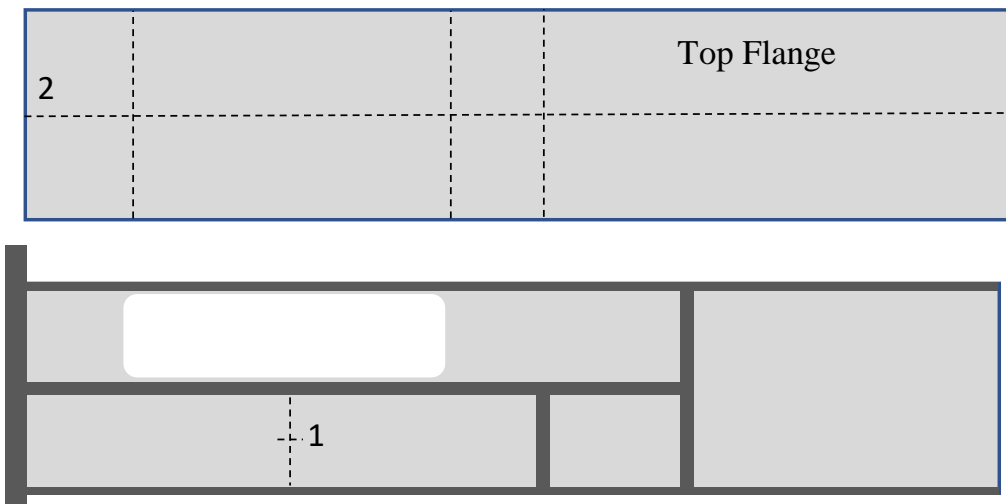



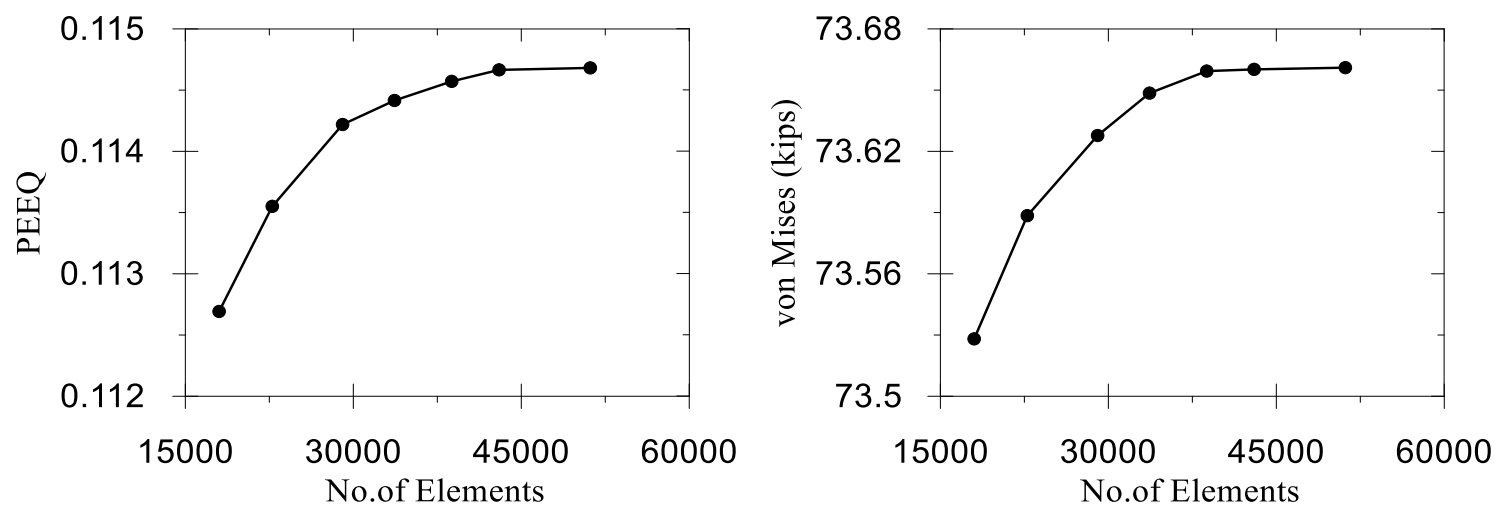

(a) W18x76 Monotonic loading at $\gamma=0.17$ (point 1)
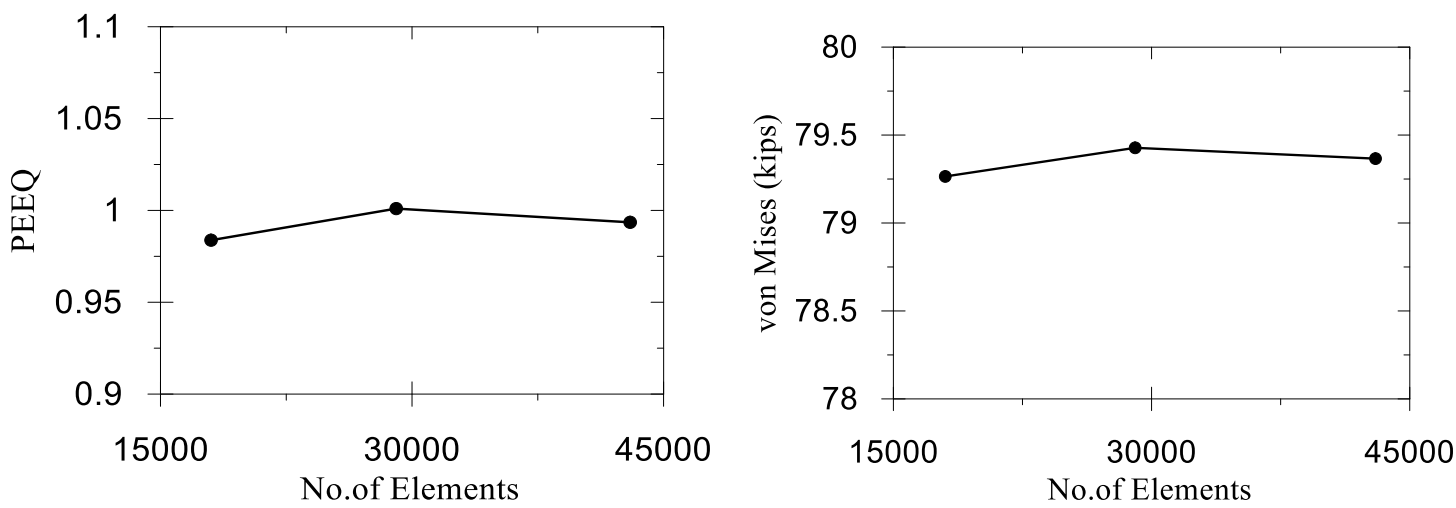

(b) $\mathrm{W} 18 \times 76$ Cyclic loading at $\gamma=0.09 \mathrm{rad}($ point 1 )
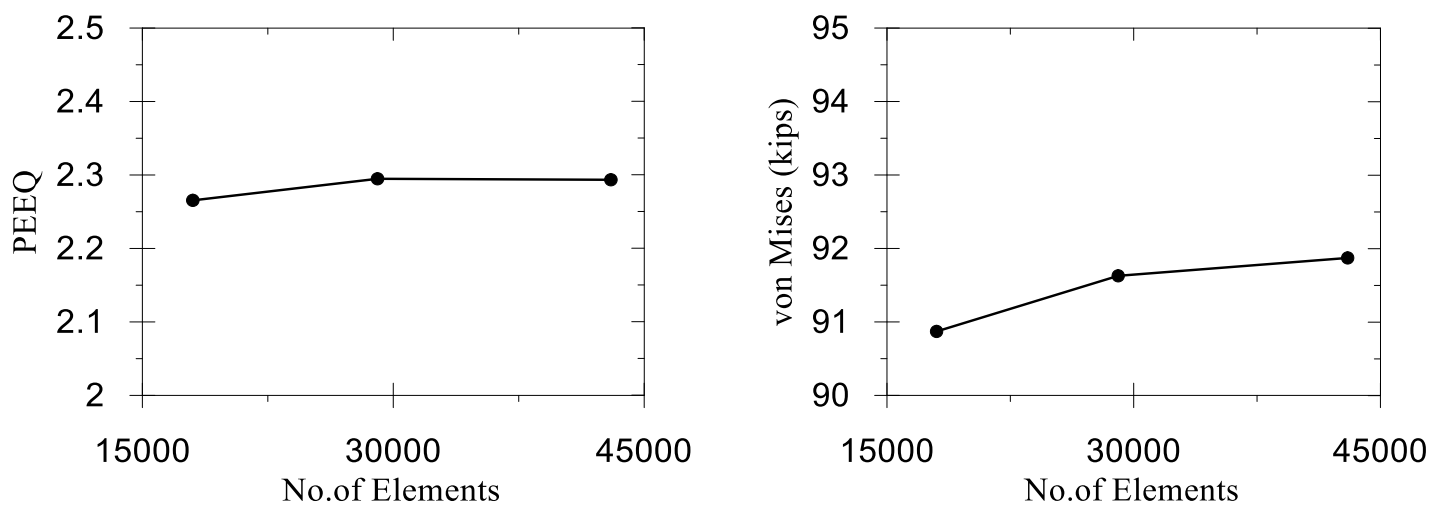

(c) W18x76 Cyclic loading at $\gamma=0.15 \mathrm{rad}$ (point 1 ) 

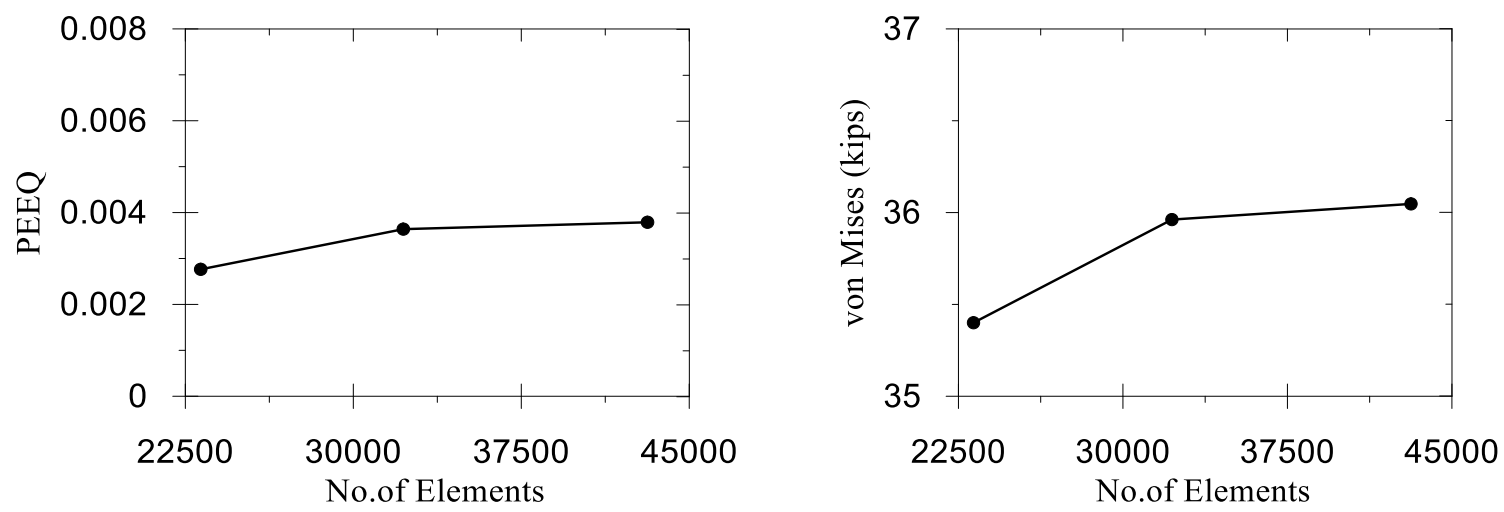

(d) W18x76 cyclic loading at 0.09 mid flange (point 2 )
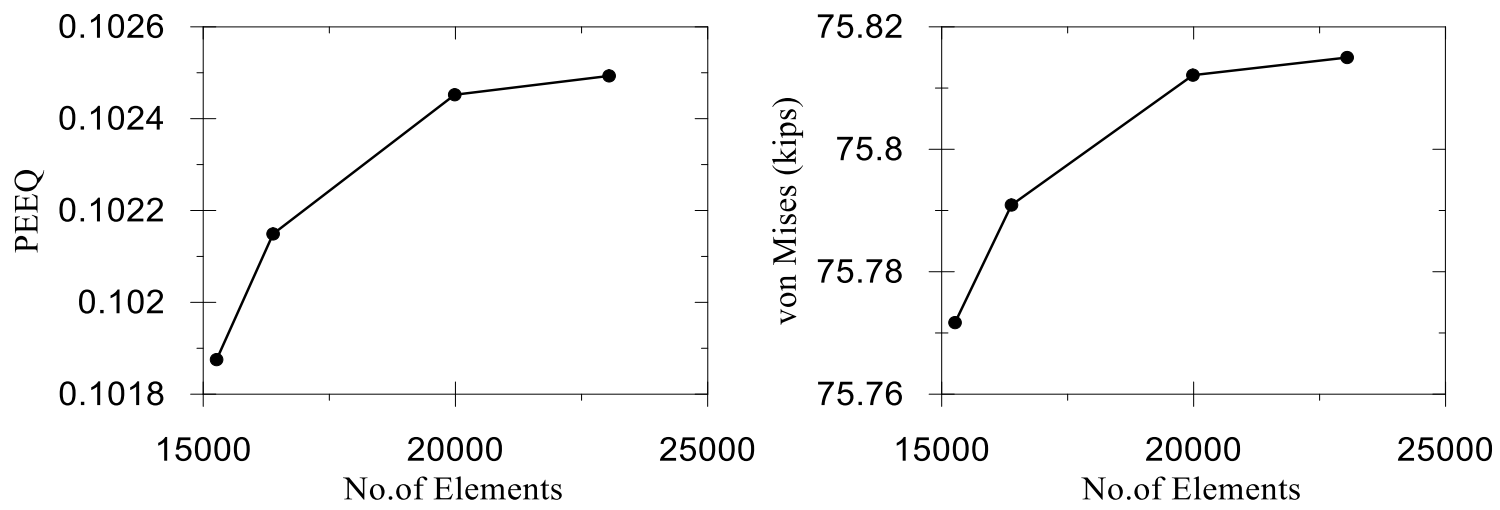

(e) W16x67 monotonic loading at $\gamma=0.17$ (point 1)
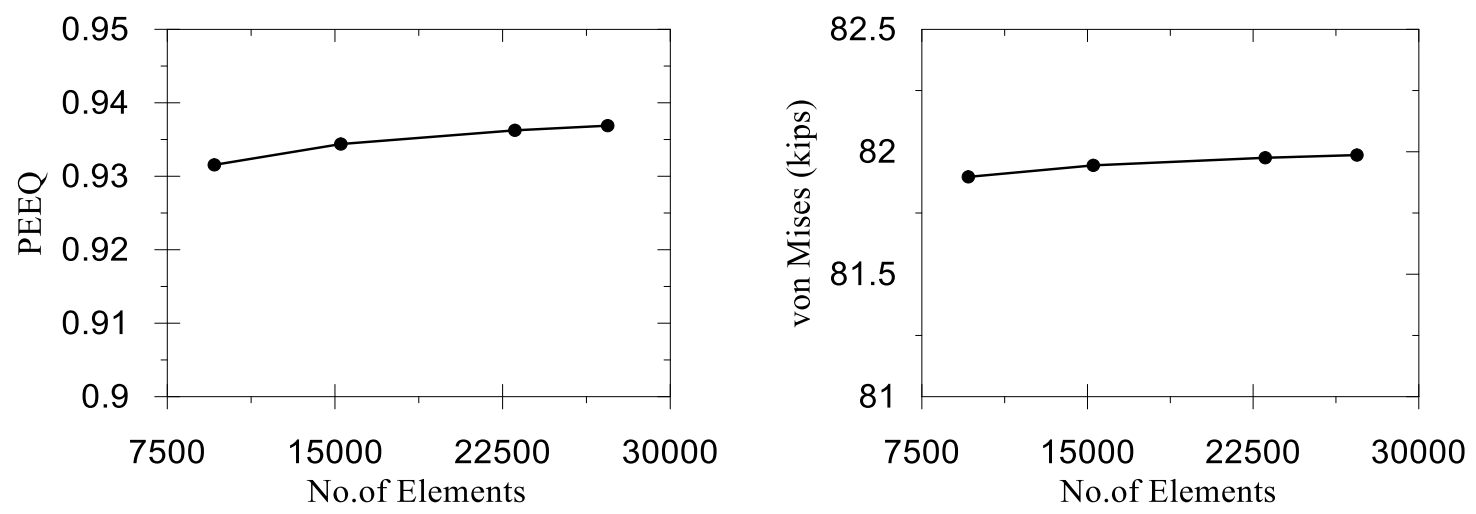

(f) W16x67 cyclic at $\gamma=0.09 \mathrm{mid}$ web (point 1 ) 

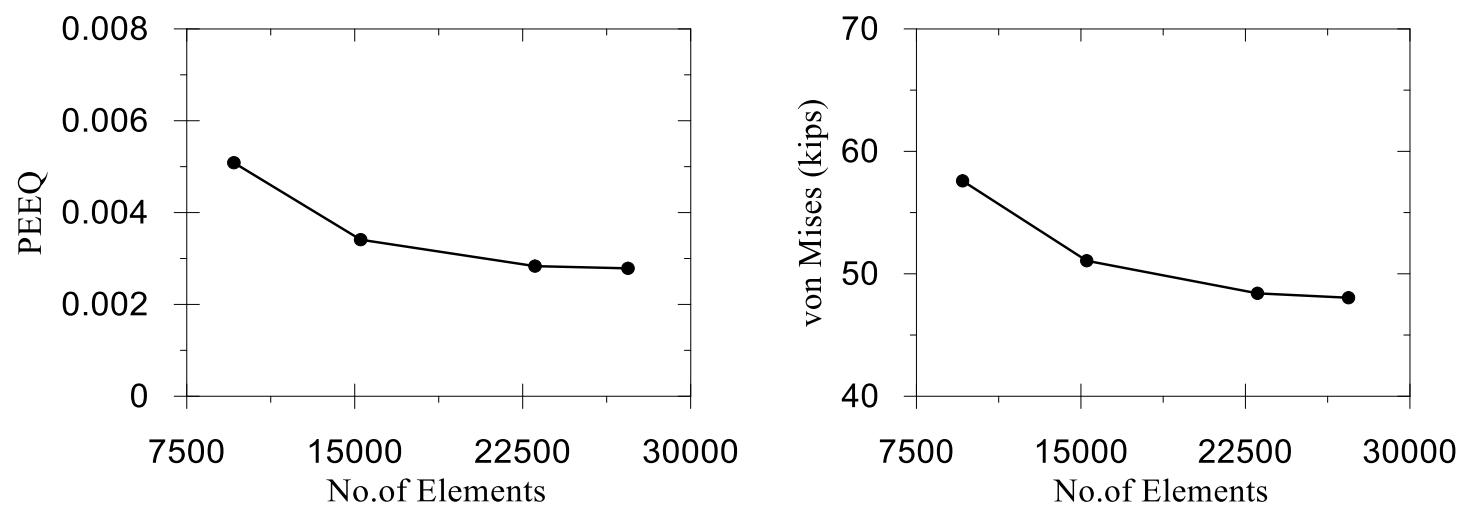

(g) W16x67 cyclic loading at $\gamma=0.09 \mathrm{rad}$ mid flange (point 2)

Figure 4.3 Convergence mesh study

\subsection{Validation of Experimental and Numerical Results}

The experimental results were analytically verified with a finite-element model, and the comparison demonstrated good agreement between them, as shown in Figure (4.4), which represents the given hysteretic shear deformation curves. Results from finiteelement analysis also showed that the model can predict buckling at different rotations for both sections. Figure (4.5) displays the web-buckled shape of the specimens at high link rotation, compared with test photos of the link buckling during cyclic testing. 


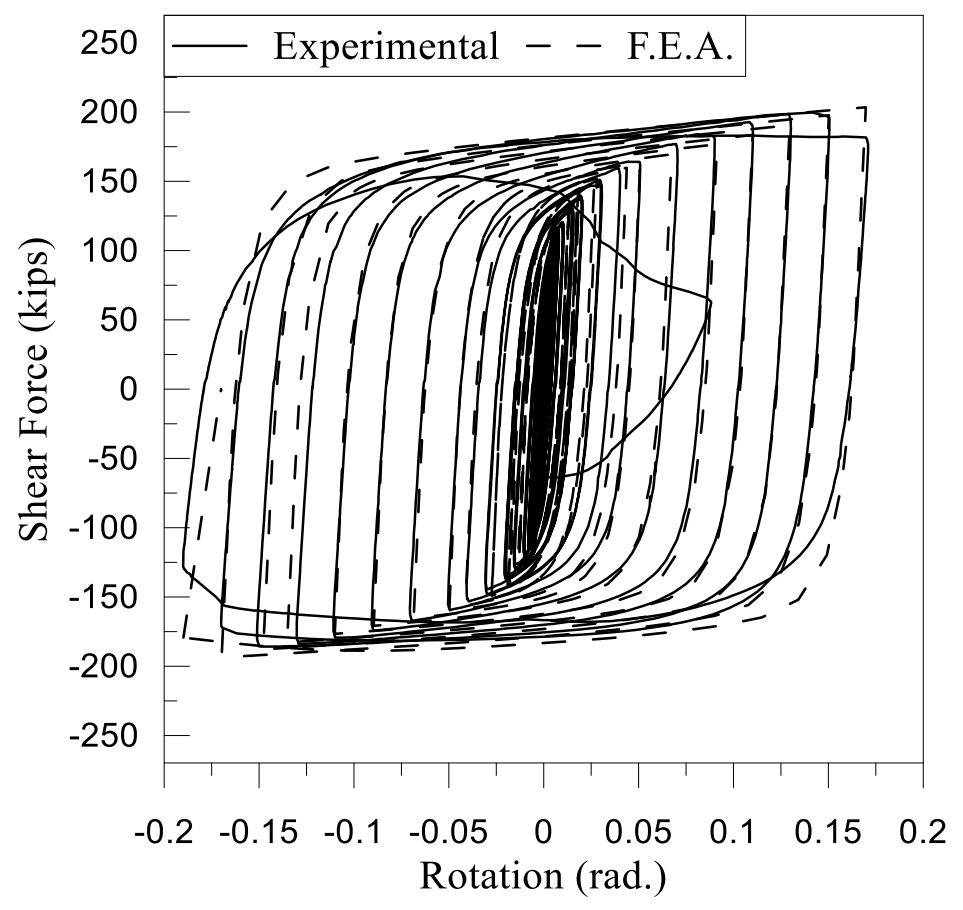

a) W16x67 Welded brace

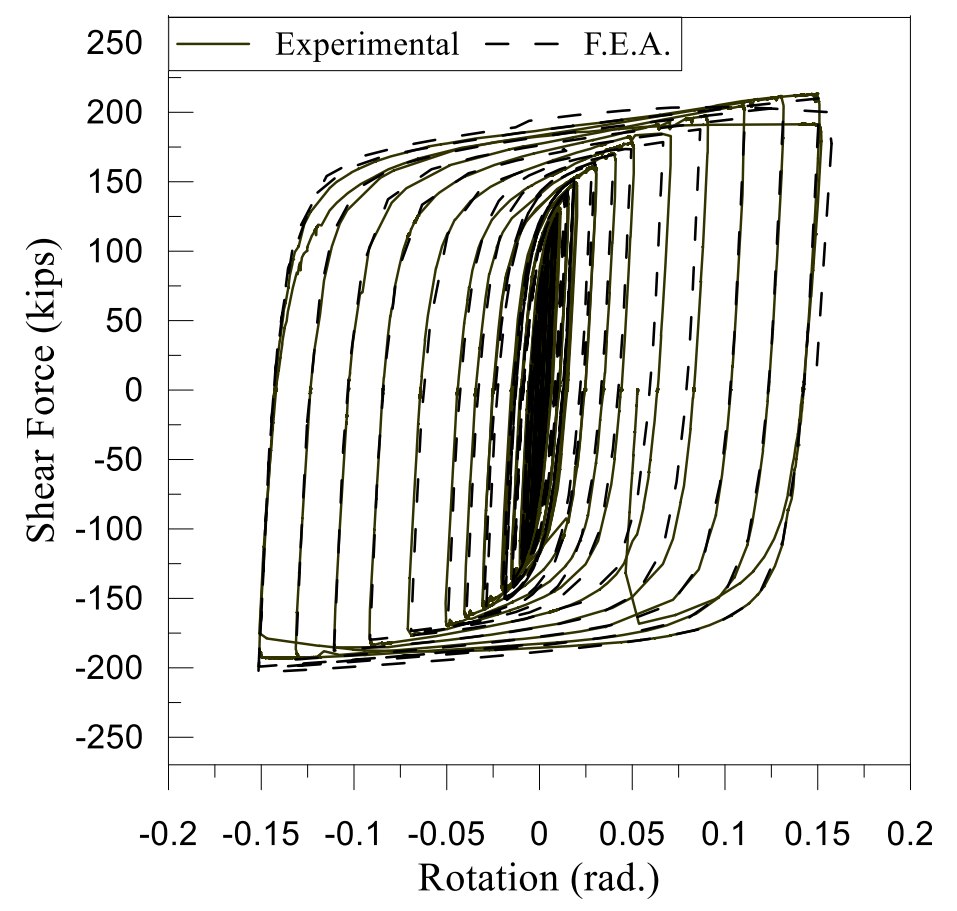

b) W18x76 Welded brace 


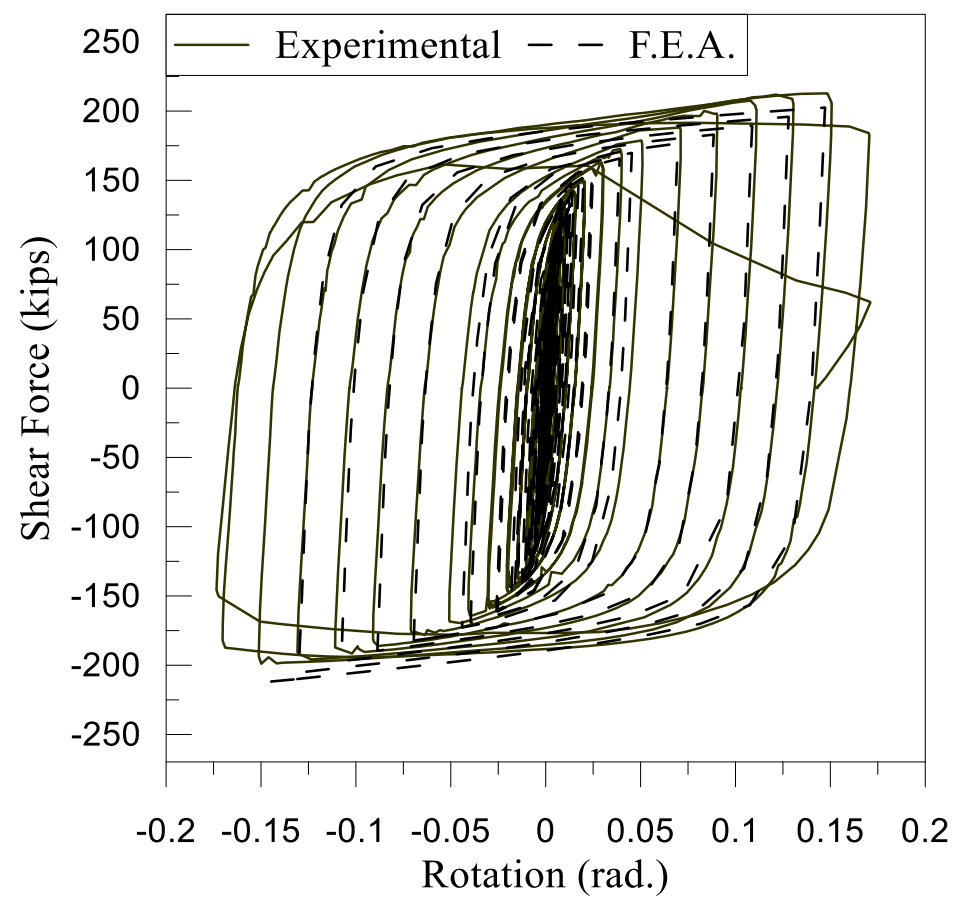

c) W18x76 Bolted brace

Figure 4.4 Link rotation-shear forces model results compared with the experimental data

The rotations of the end panels, panels $\mathrm{ABEF}$ and $\mathrm{CDGH}$, were also verified and compared using FEA. The graphs in Figure (4.6) represent the backbone of the theoretical hysteresis compared to the experimental curves. In addition, Table (4.2) summarizes the contribution percentages of the effective panel, BCFG, from both the experimental and numerical model at a rotation equal to 0.09 rad. Obviously, the specimen without transition zone cracks, W16_Welded, has close percentage contribution while the section with transition zone cracks, W18_Welded, has much larger deference due to the effect of the cracks on the rotation stiffness of the end panels as discussed in chapter 3. 

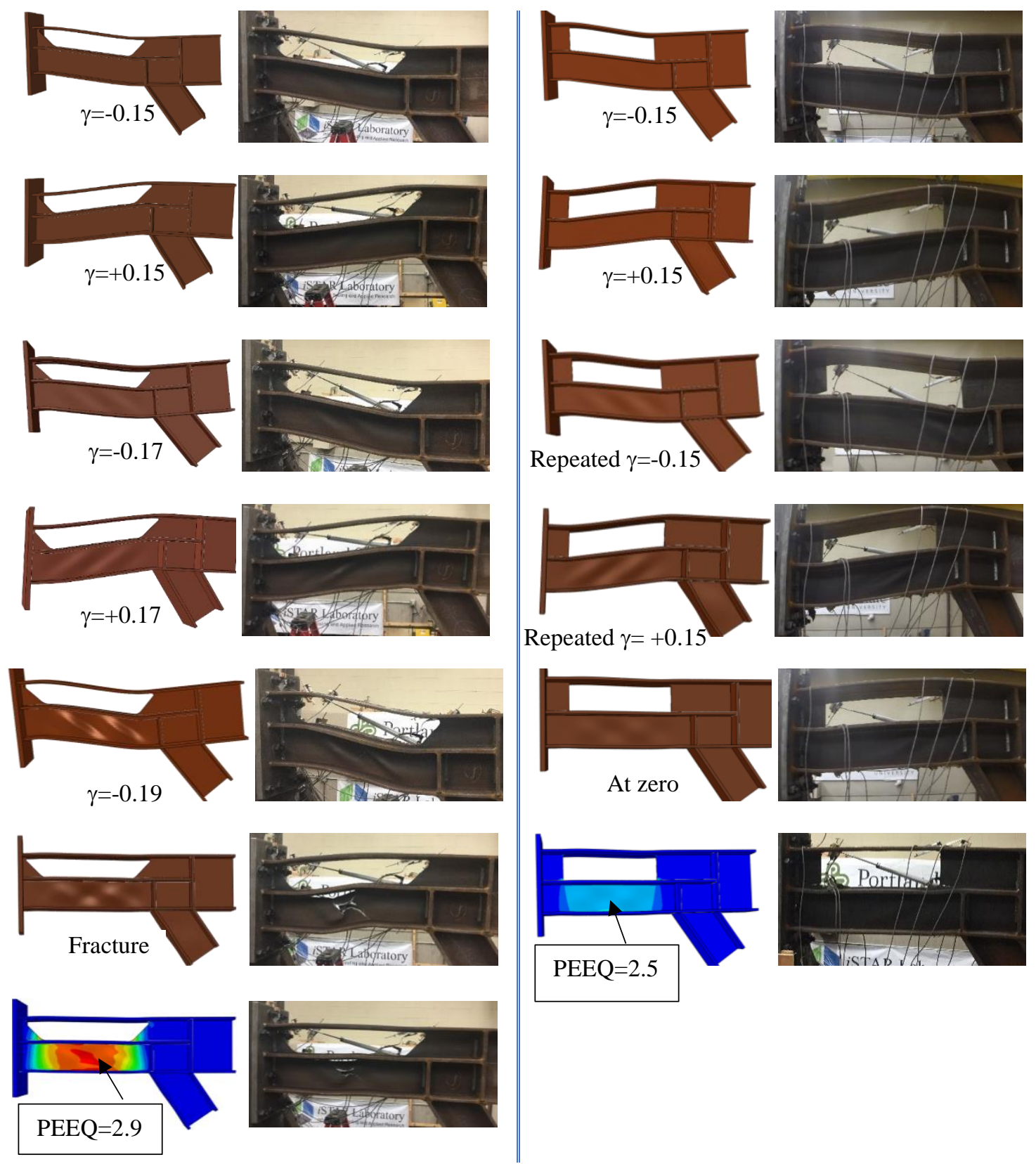

(a) W16x67 Welded brace

(b) W18x76 Welded brace 


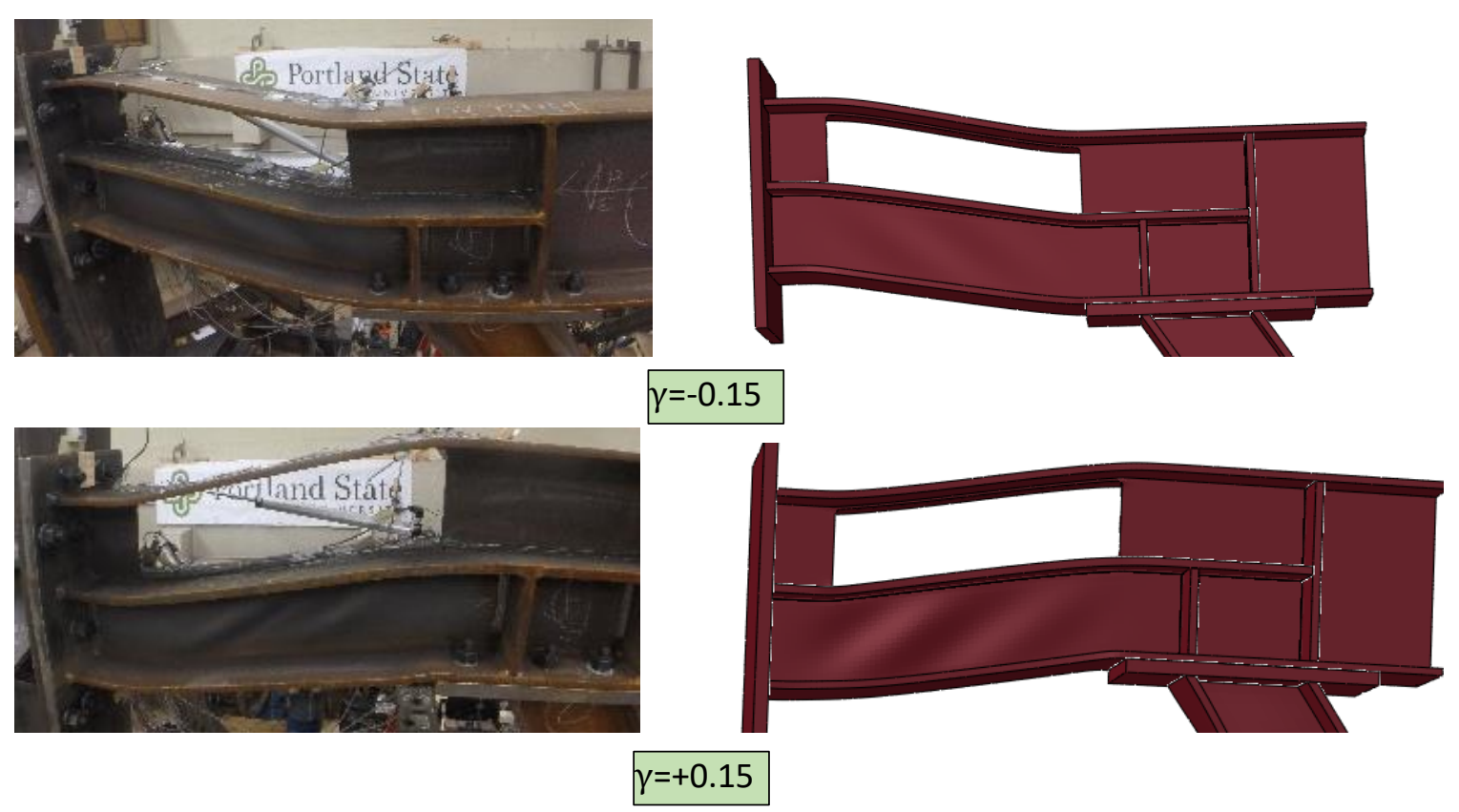

c) W18x76 Bolted brace at $\gamma=-0.15$ and $+0.15 \mathrm{rad}$

Figure 4.5 Links deformation for FEA vs experiments
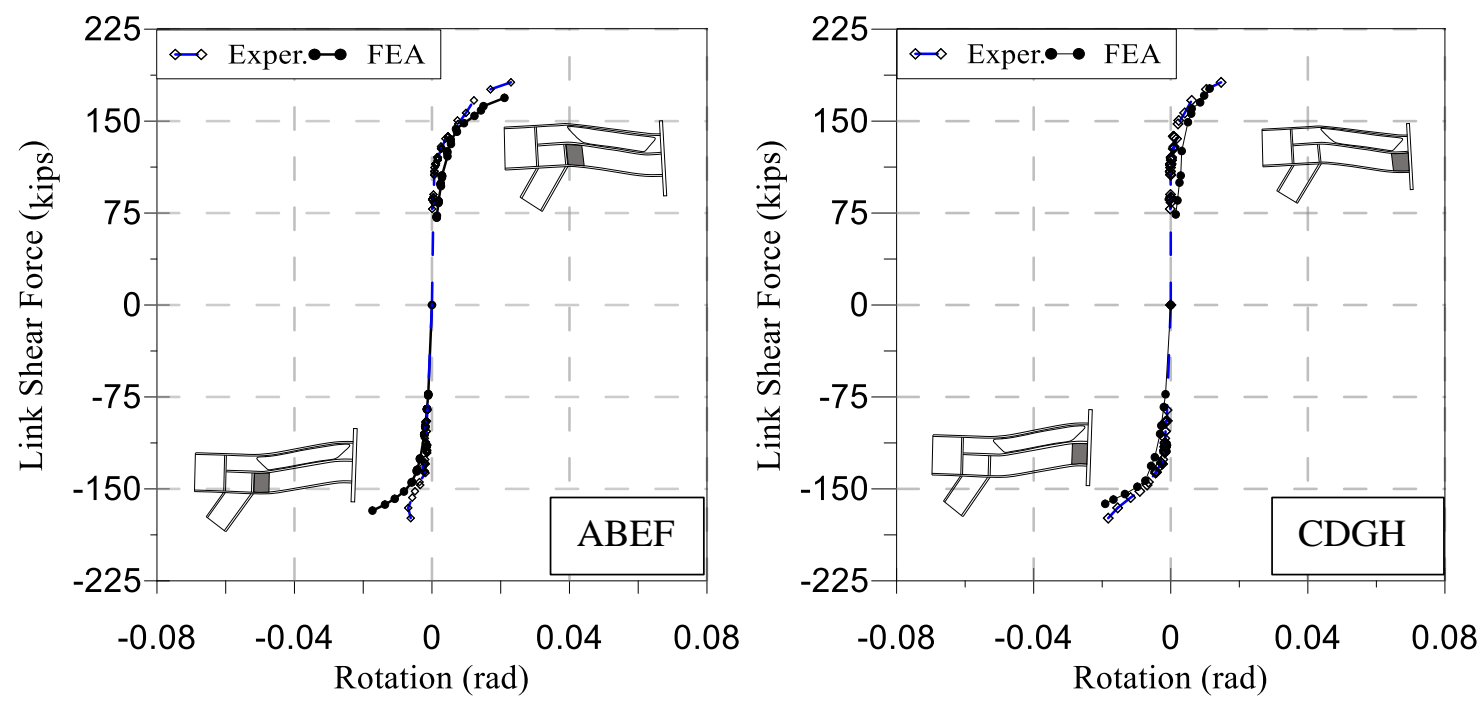

Figure 4.6 Comparing end panels' rotations for experimental and numerical analysis (W16_welded) 
Table 4.2 Summary of contribution percentage of effective panel to the total link rotation at $\gamma=0.09 \mathrm{rad}$

\begin{tabular}{|c|c|c|}
\hline Section & Experimental & FEA \\
\hline W16_Welded & $92.3 \%$ & $93.75 \%$ \\
\hline W18_Welded & $84 \%$ & $98 \%$ \\
\hline
\end{tabular}

\subsection{Geometry Imperfections}

Web local buckling is the main damage that might develop in the shear link. In contrast, flange local buckling is the primary damage in the flexural link type. Stability analysis is required in structural steel design by modern design standards. Geometric imperfection is a good method for stability analysis. It would be very useful to study the effect of initial web buckling on the behavior of the link, since the configuration of TEBF has no intermediate transverse web stiffeners. First, eigenvectors must be extracted from the modal analysis. Then, initial local imperfection can be defined by applying a scale local buckling from Eigen mode, as shown in Figure (4.7), or initial crookedness (out-of-straightness).

In this analysis, the initial buckling was scaled equal to $\mathrm{d} / 150$ based on previous research (Seif and Schafer, 2013; Kim and Lee, 2002). The imperfection scale factor was 0.12, as shown in Figure (4.7c), which also shows the section with large scale factor of geometry imperfection to display exaggerated buckling Figure (4.7b). Looking at Figure (4.7a), which describes the Eigen modes of the T-EBF, mode 41 had web buckling, which can be used in imperfection analysis. Figure (4.7h) compares the analysis results from the two models using FEA, one with initial geometrical imperfection and the other without, and illustrates the deformation and strain distribution of the link at a rotation of 
$0.15 \mathrm{rad}$. The shear force versus the link rotation curve drawn from this analysis proved that T-EBF can achieve a stable hysteretic behavior. The results have shown that the initial imperfection has an influence on the web buckling at a high rotation, affecting the shear strength of the link.

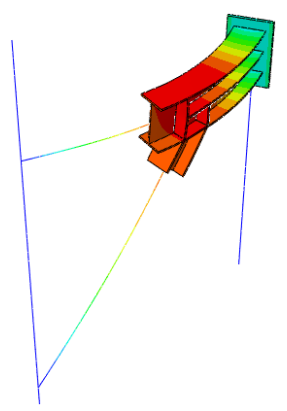

Mode shape 1

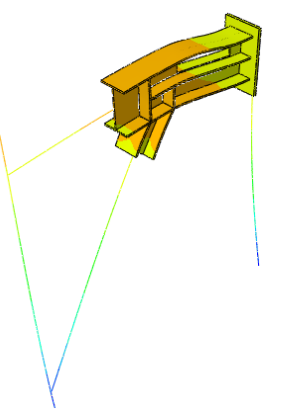

Mode shape 2

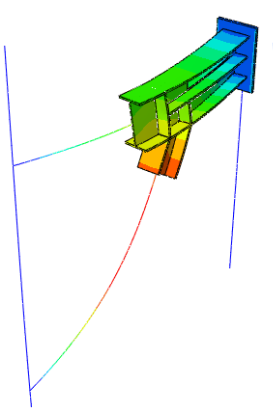

Mode shape 3

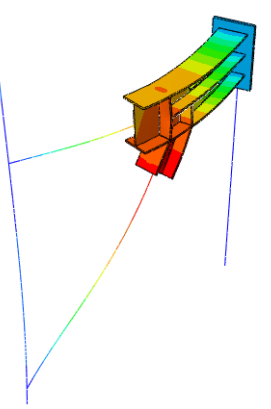

Mode shape 4

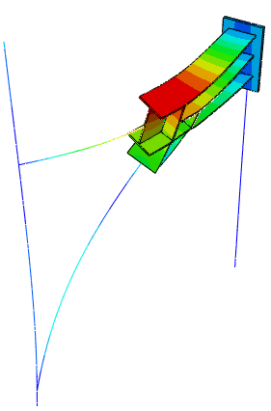

Mode shape 5

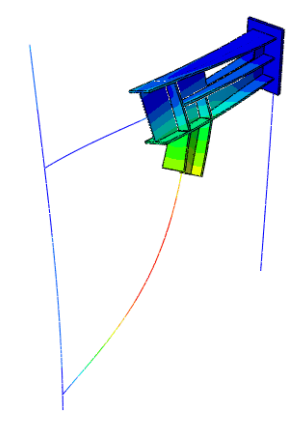

Mode shape 6

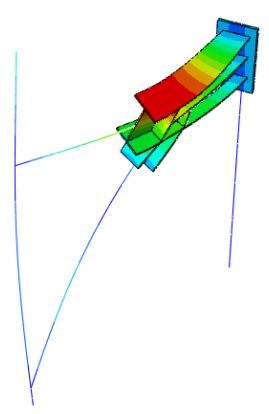

Mode shape 7

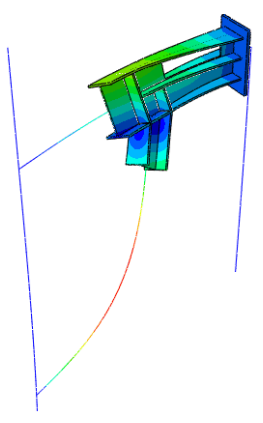

Mode shape 8

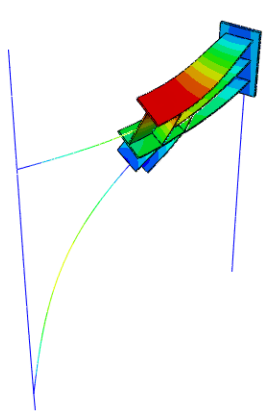

Mode shape 9

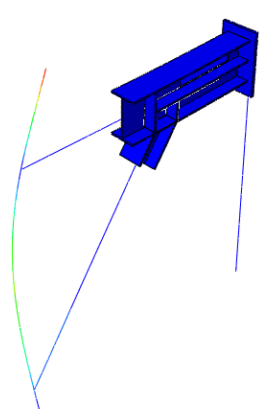

Mode shape 10

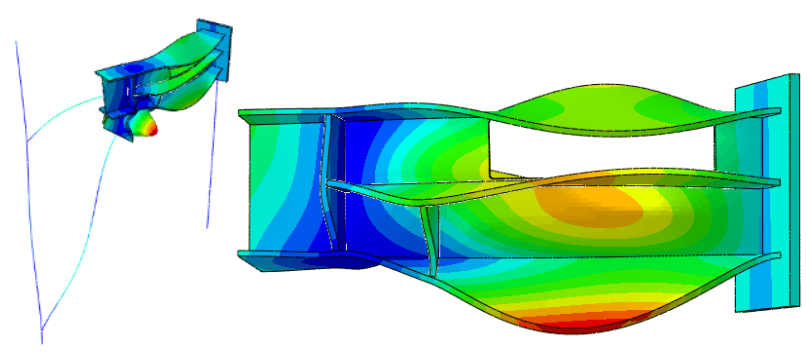

Mode shape 41

(a) Mode Shapes 


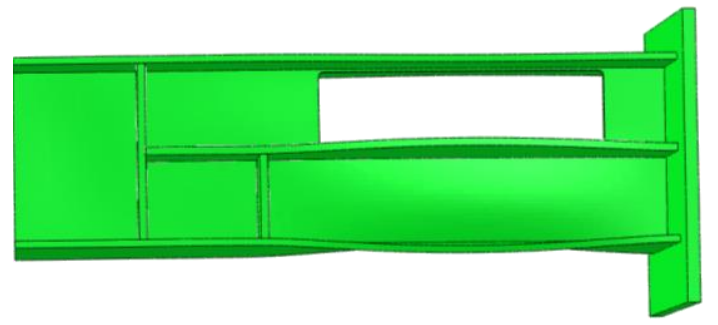

(b) Initial imperfection [Large scale factor]

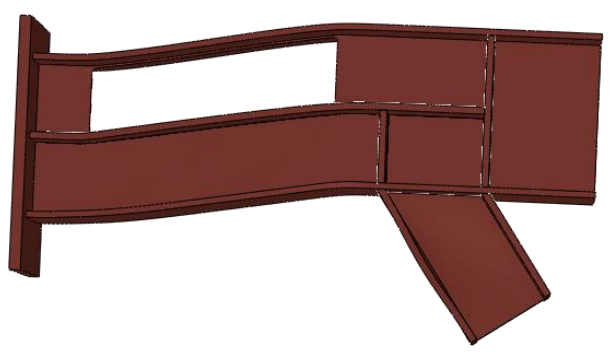

(d) Link without initial geometrical imperfection at $+0.15 \mathrm{rad}$

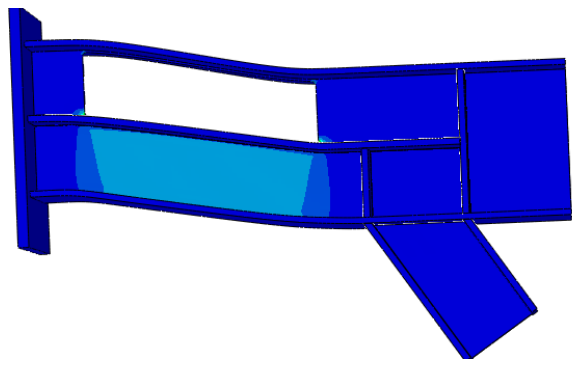

(f) PEEQ distribution for link without imperfection at $-0.15 \mathrm{rad}$

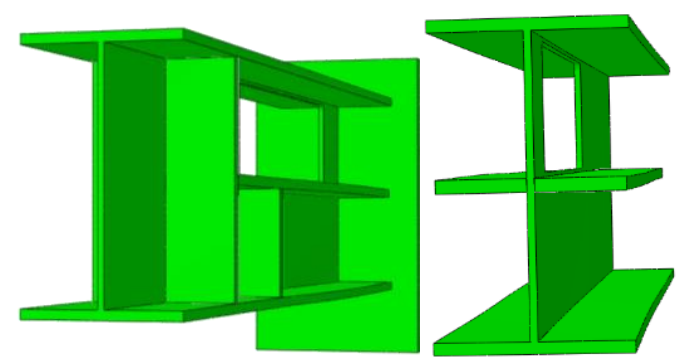

(c) The applied Initial imperfection [scale (d/150), 0.12]

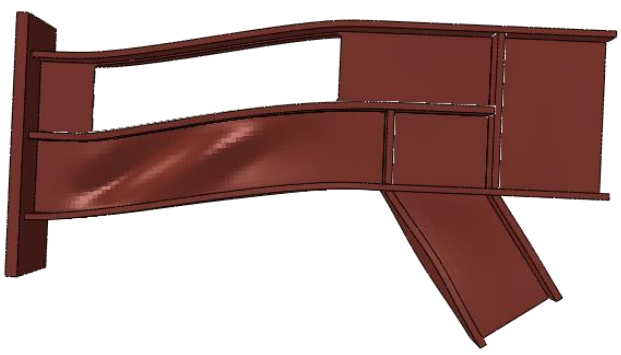

(e) Link with initial geometrical imperfection scaled to $(0.12)$ at $\gamma=+0.15 \mathrm{rad}$

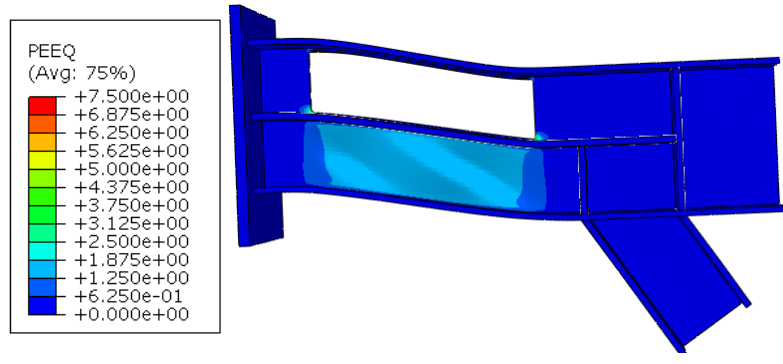

(g) PEEQ distribution link with initial geometrical imperfection at $-0.15 \mathrm{rad}$ 


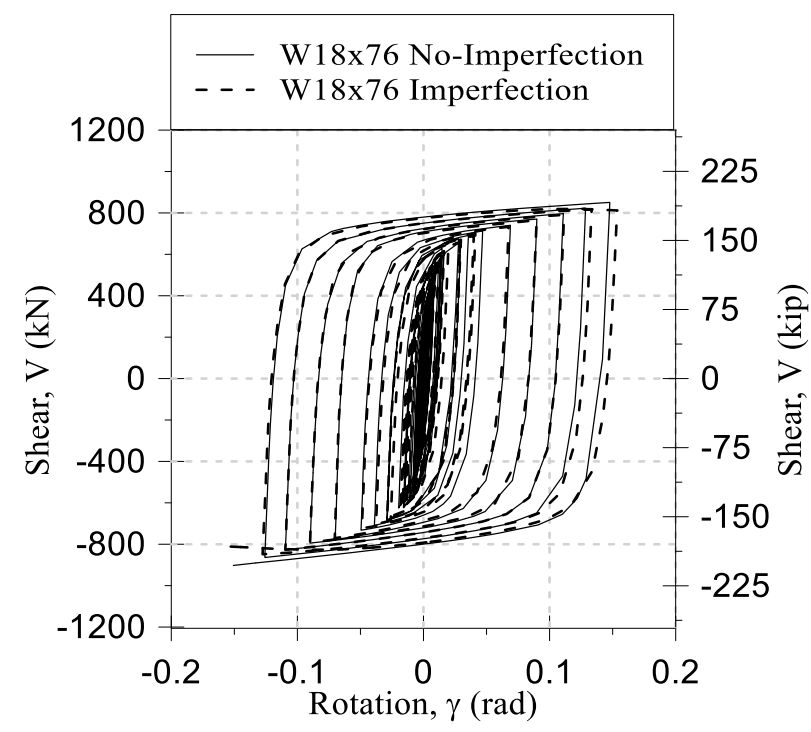

(h) Link Shear-Rotation curve

Figure 4.7 Effects of initial geometrical imperfection

\subsection{Bolted Brace to Link Simulation Using Finite Element Method}

Among several EBF links used in the Christchurch hospital parking garage, two frames were observed fractured under the same circumstances. The main observation of these two fractured EBF is that the misalignment of the stiffeners with the brace flange caused stress concentration in the offset area. Other EBF in the building performed well and dissipate the earthquake energy through the link inelastic deformation that achieved by significant yielding but without any signs of fractures.

This part discusses the development of an alternative brace to link connection that can be bolted into place and that confines damage to the link. The proposed connection is expected to result in reduced the stress concentration in the beam flange that might occurs due to detailing error during installation and fabrication. 
One of the characters of the new brace to link connection is using a thick plate which is bolted to the beam flange and welded to the brace. The thick plate can increase the stiffness of the connection area and distribute the stress along the plate length. The main objective of this part of the research is to investigate applying the bolted connection on the real fractured EBF that occurred during the Christchurch earthquakes and investigate the bolted brace to link connections on the ductility of EBF with offset of the stiffeners (the brace flange to beam connection point with the link end stiffeners). The plate thickness is also a parameter used in this part to investigate the effectiveness of the thickness. However, the analysis does not intend to discuss the effect of the plate on the general behavior of the link and it is focusing on the ability of the plate to reduce the stress on the offset area. Causing of the fractures was misalignment of the web stiffeners with the brace flange. Therefore, the analytical models consist the original link and a modified link that using thick plate in the brace connection. The sections of the beam, link and the brace were the actual sections that were used in the actual frame. The dimensions, geometry and material properties, Table (4.3), are obtained from previous study (Imani et al, 2015) which is adapted by AISC seismic design provision (ANSI/AISC 341-16, 2016) to warn designer about these types of EBF failure. Based on material behavior, Imani suggested that when PEEQ is equal to $19.8 \%$ the elements reach the fracture strain. The column and the brace were modeled to be elastic during the analysis. The link and the beam material is a bilinear material property. To simplify the model, half the frame was modeled by taking the advantage of the symmetry. The boundary conditions for model simulation are shown in Figure (4.8c). They contain: 
Pinned support at the column base with no movement in all the three directions; lateral support to prevent sideway movements and restriction of the movement along the symmetrical plane in vertical direction. Convergence study was conducted by examining the mesh density versus cumulative plastic strain PEEQ and then the element size was selected to be 0.2 inches. Nonlinear monotonic analysis, which was used in previous study, is a simple and efficient way to investigate the effect of the misalignment the web stiffeners. Lateral pushover, displacement control, was assigned to apply on the frame in two opposite directions.

Table 4.3 Simple bilinear steel material used in FEA (Imam, 2015)

\begin{tabular}{|c|c|c|c|c|}
\hline & $\mathbf{E}$ & $\mathbf{v}$ & $\boldsymbol{\sigma}_{\mathbf{y}}$ at strain 0.2\% & $\boldsymbol{\sigma}_{\mathbf{u}}$ at strain 15\% \\
& $\mathbf{M p a}$ & & $\mathbf{M p a}$ & $\mathbf{M p a}$ \\
& $(\mathbf{k s i})$ & & 350 & $(\mathbf{k s i})$ \\
\hline Bilinear Material & 200,000 & 0.3 & $(50)$ & $(65)$ \\
& $(29000)$ & & - & - \\
\hline Elastic & 200,000 & 0.3 & & \\
& $(29000)$ & & & \\
\hline
\end{tabular}
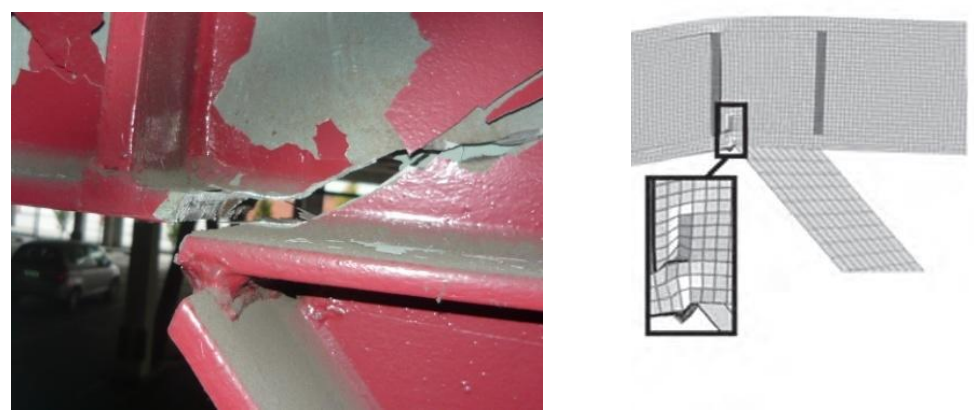

a) Imani et al. 2015, Kanvinde et al. 2014 


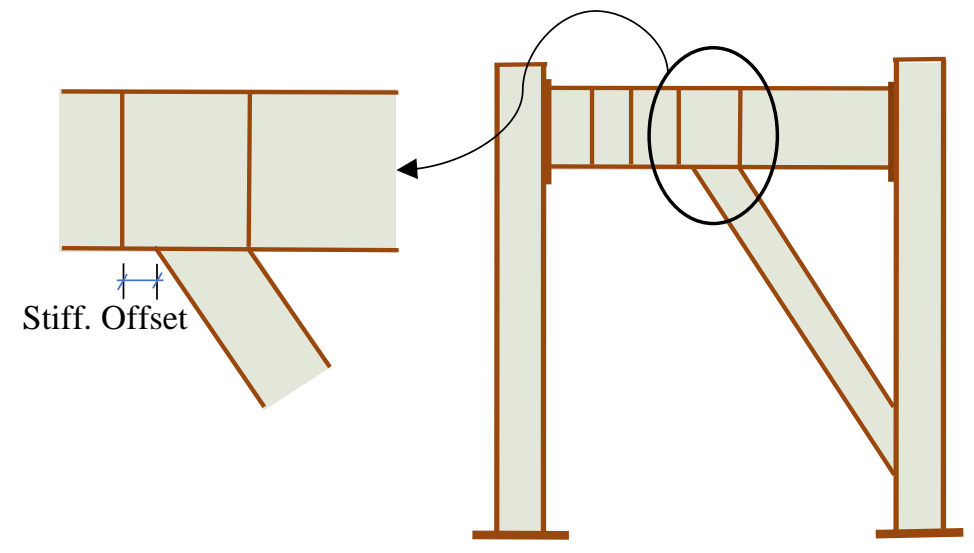

b) Stiffener Misalignment

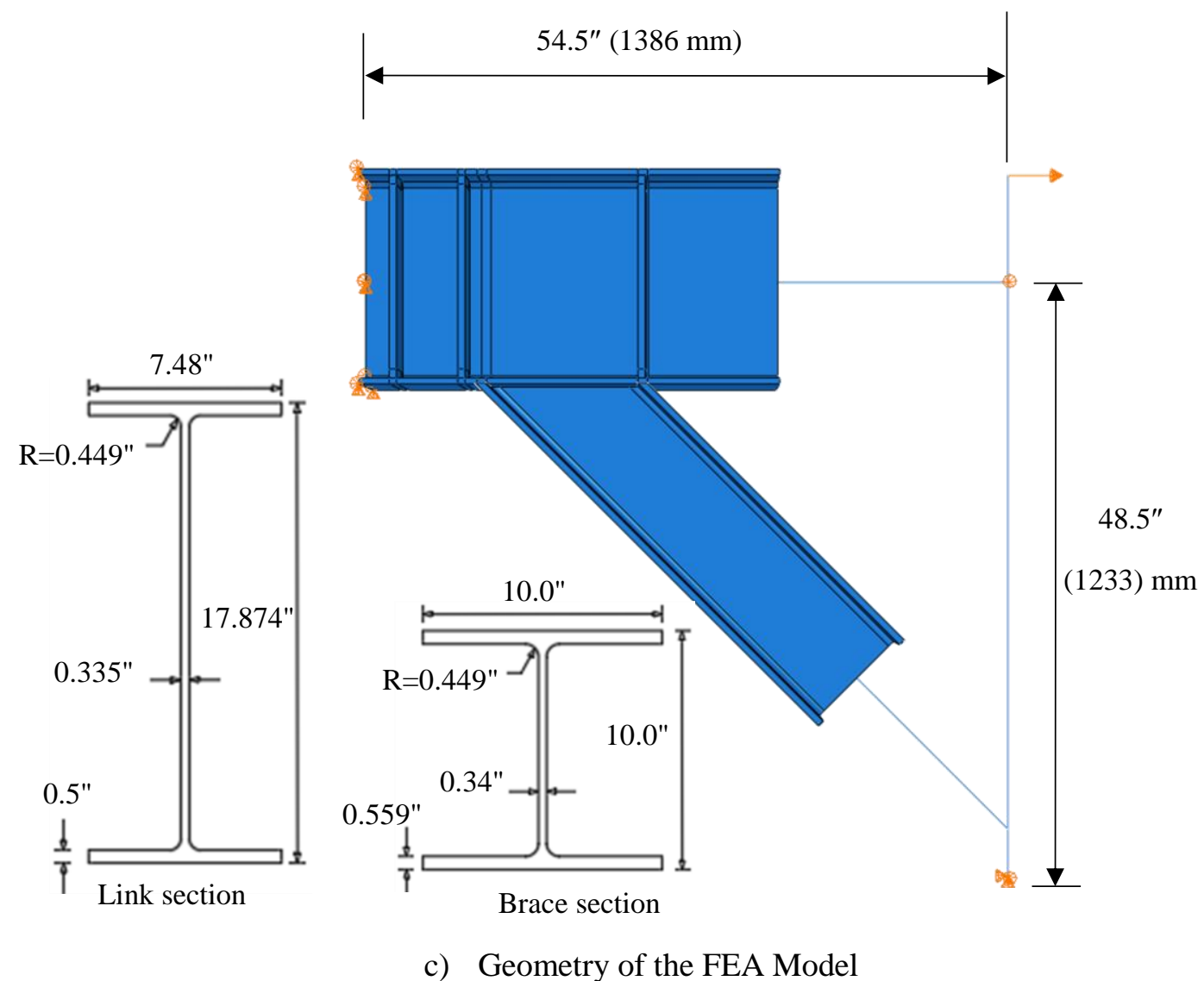




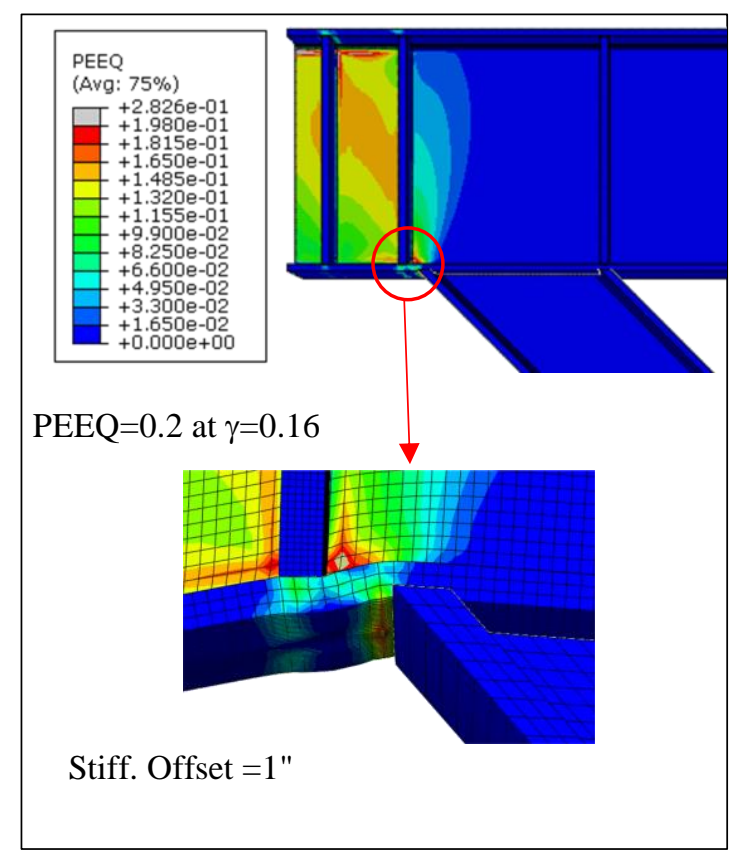

PEEQ for Actual EBF

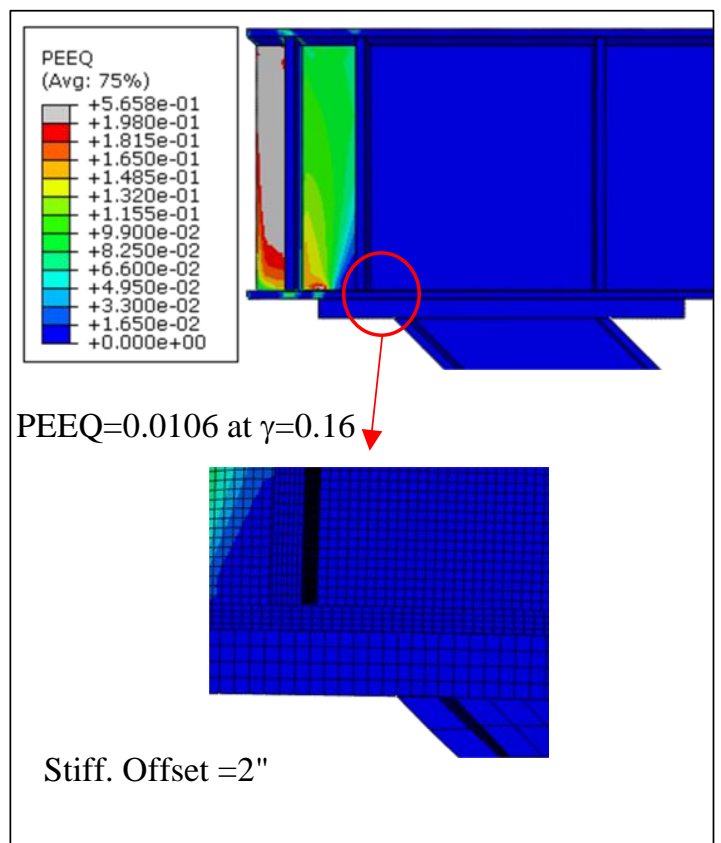

PEEQ for Model with Thick Plate (1.25")

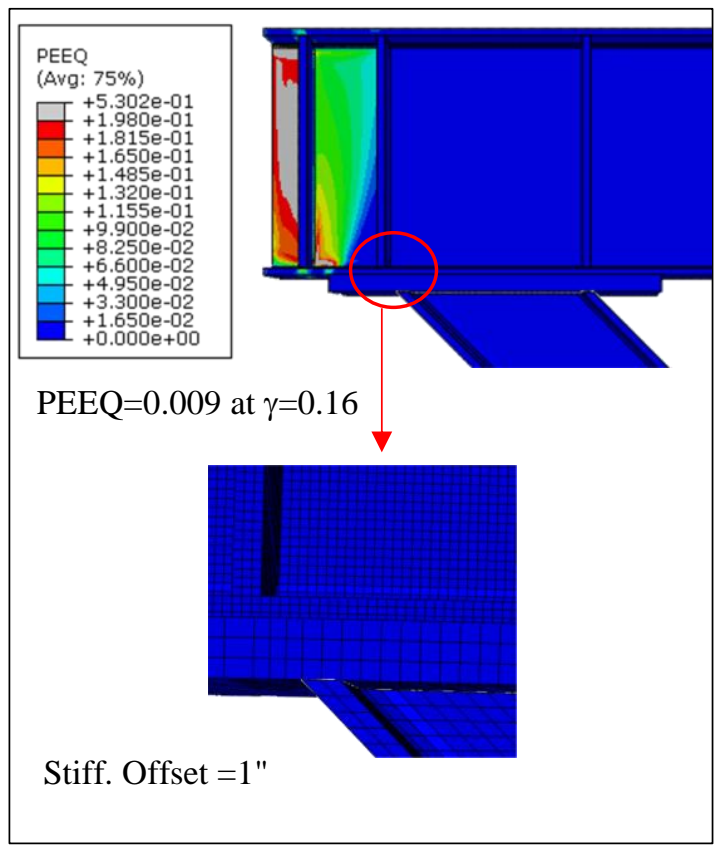

PEEQ for Model with Thick Plate (1.25")

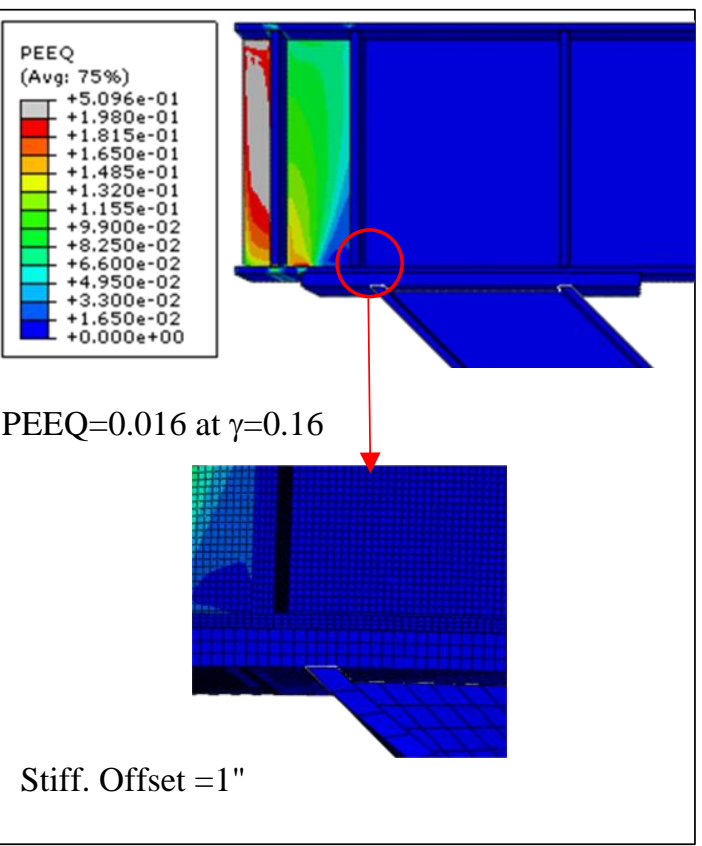

PEEQ for Model with Thick Plate (1.0") 


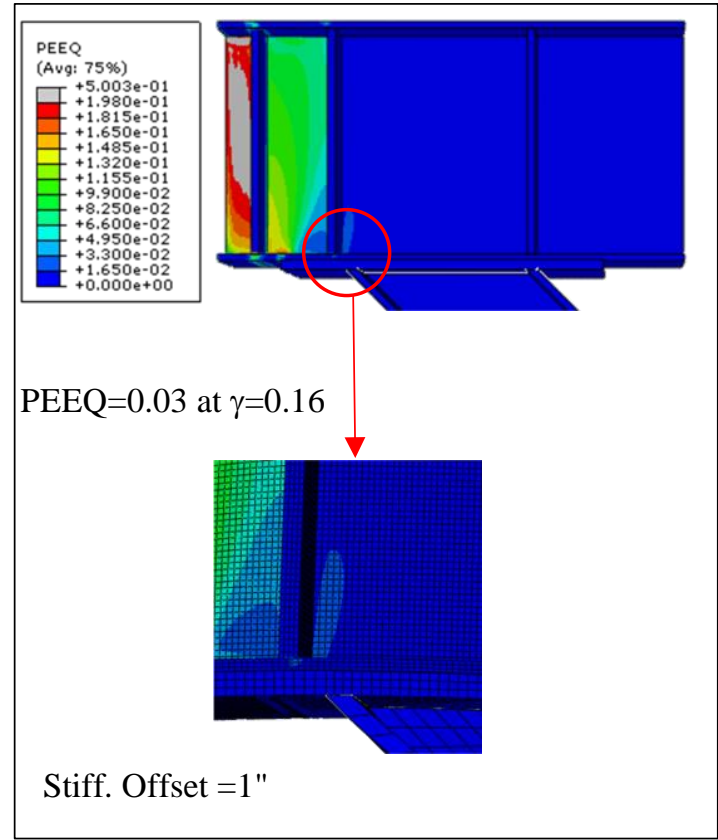

PEEQ for Model with Plate (0.75")

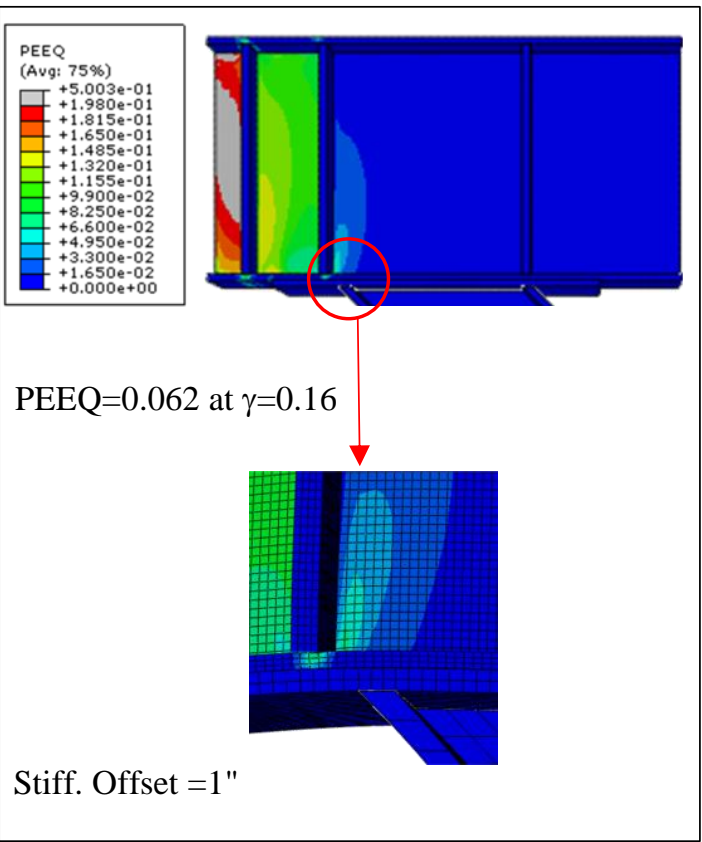

PEEQ for Model with Plate (0.5")

d) FEA results of different cases

Figure 4.8 EBF described in the Christchurch Hospital Garage (Imani et al. 2015, Kanvinde et al. 2014)

Analytical results from using a thick plate at the brace-to link-connection, as described in Figure (4.8d), show that plastic strain can reduce near to zero at very high link rotation angle. In addition, the suggested connection can move the plastic hinges away from the connection to inside the link which means reduce the stresses near the connection during earthquake events. Therefore, the bolted brace connection represents a promising solution since the primary target in the design of EBF is to restrain the plastic strain within the link while retaining the other components including beam outside the link in the elastic stage. 


\section{Chapter 5: Parametric Study on Key Dimensions of T-EBF}

\subsection{Introduction}

EBF was presented at the end of the 1970's as new frames were designed according to evolving seismic design philosophy and performance criteria to allow yielding within the link and to design the remaining components to stay elastic (Roeder and Popov, 1977).

As this study suggests, more control over the link strength and its properties can be achieved through making an opening in the web link and producing a system with excellent seismic performance. Therefore, even deep beams can have limited link strength capacity and can sustain the required inelastic rotation. In addition, a reduction of the web area is an idea similar to the concept of strengthening the area near connections to ensure the plastic hinge will form away from the connections so that columns, beams, and the connections can behave elastically under seismic loads.

Applying the concept of reducing the section area on the shear links of EBFs in either the web or the flange has been studied before (Prinz and Richards, 2009), (Berman et al. 2009). Control over the strength of EBF links allows for use of different size web openings within the link length (e) to maintain architectural requirements and, at the same time, can maintain the lateral stiffness of the frame and control its strength. Using a smaller effective link length $\left(e^{\prime}\right)$ than the entire link length (e) results in larger inelastic rotation at the same story drift. 


\subsection{Control over EBF Strength}

The primary appealing features of T-EBF is the capability to tune their strength according to seismic design requirements. Since the size of the link in EBF governs the design of the entire structure, using the same link and beam section can result in a significantly oversized design resulting in the use of more materials than should be required and increased construction costs.

In addition, the amplified forces resulting from the difference between the design forces and the capacity of the selected section can cause higher base shear than required in design. More control over the strength of the link while achieving the desired rotation capacity and the architectural advantages of EBF can direct efforts to improve the link behavior. Reducing the web area by cutting apart has shown an experimentally promising solution to design T-EBF link to link-column connections.

The link properties can be controlled in both the length of the effective link e' and effective depth $\mathrm{h}_{\mathrm{w}}$. Controlling the effective depth and length would allow the designers to:

- Use different opening sizes to accommodate architectural requirements.

- Use various deep wide flange sections that cannot otherwise serve as links in EBF.

- Use the same section size for floors in tall, multistory buildings along with tuning the strength for ease of construction and manufacture. 
The results from this chapter will serve as a design guideline recommendation to carry out the T-EBF concept.

\subsection{Parameters Used in Design of T-EBF}

The seismic resistance design requires EBFs to safely sustain the desired rotation. Cyclic analysis can be used to examine the effectiveness of T-EBF parameters on the link behavior. Parameters such as end distance, effective depth, stiffener thickness, and fillet radius will be evaluated using FEA described in the previous chapter to better understand their effects on the behavior of T-EBF. The primary design parameters of T-EBF, ED, e', $\mathrm{h}_{\mathrm{w}}$, and $\mathrm{t}_{\mathrm{hs}}$ are used to control the link geometry, as shown in Figure (5.1).

ED- End distance;

$h_{w}-$ Section effective depth

$\mathrm{W}_{\mathrm{s}^{-}}$Weld stub;

$t_{\text {hs- }}$ Thickness of horizontal stiffeners;

$\mathrm{t}_{\mathrm{vs} \mathrm{s}^{-}}$Thickness of vertical stiffeners.

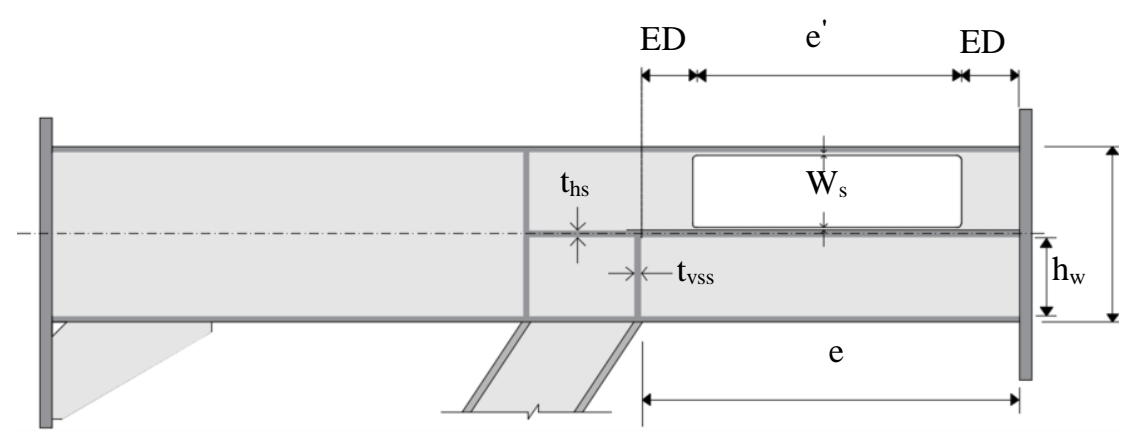

(a) T-EBF link 


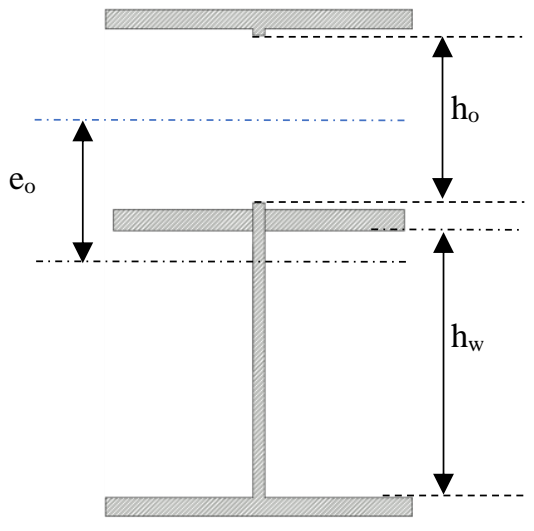

(b) Cross-section

Figure 5.1 Parametric study

In the following sections, an exclusive analysis will be displayed to address these parameters in detail. The W18x76 section was experimentally tested and will be used for this purpose by remodeling the link geometry. All the links used in this research are shear links.

\subsubsection{End Distance (ED)}

The first parameter is the end distance or end panels as they play a crucial role in determining the link length. The end panels can work to strengthen the connection area through ensuring that the inelastic action occurs within the effective panel and away from the column and brace connections, reducing plastic strain demand. Nine models were used to study the stability of the model by changing ED from zero to 9 inches, as shown in Figure (5.2). Table (5.1) contains the model dimensions and properties, including plastic shear and moment. The results are presented in terms of story drift-base shear, link 
rotation-shear force, plastic strains at the interested points, angle story drift, and distribution of stresses and strains in the links.

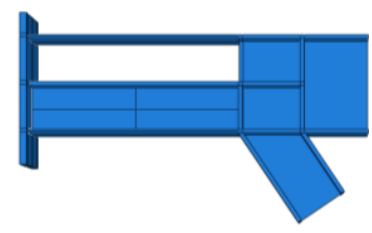

(a) $\mathrm{ED}=0$

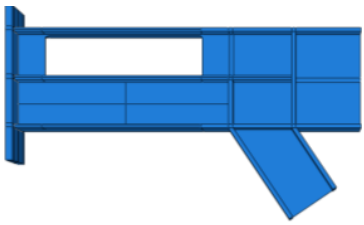

(e) $\mathrm{ED}=5$

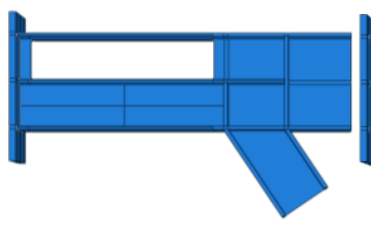

(b) $\mathrm{ED}=2$

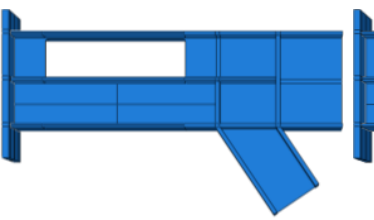

(f) $\mathrm{ED}=6$

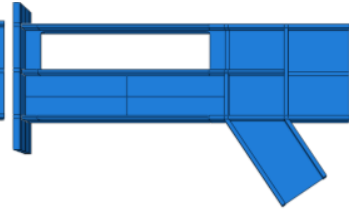

(c) $\mathrm{ED}=3$

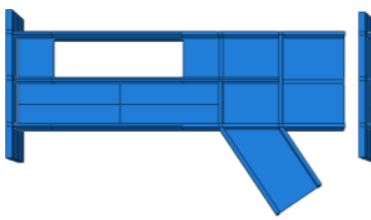

(g) $\mathrm{ED}=7$

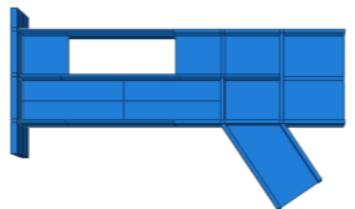

(i) $\mathrm{ED}=9$

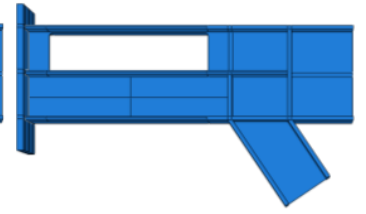

(d) $\mathrm{ED}=4$

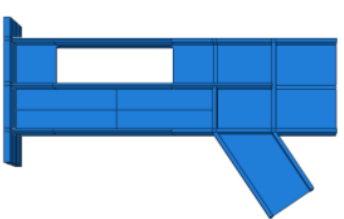

(h) $\mathrm{ED}=8$

Figure 5.2 Models ED parameter

The shear links used to investigate ED parameter ranged from 0.64 to 0.35 and length from about $40^{\prime \prime}$ to about $22^{\prime \prime}$. The plastic properties of the links are the same as the opening depths are equal. 
Table 5.1 Tuned section W18x76 properties, ED Models

\begin{tabular}{|c|c|c|c|c|c|c|c|}
\hline 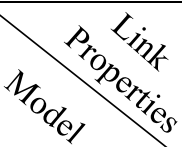 & $\begin{array}{l}\mathrm{h}_{\mathrm{w}} \\
\text { (in.) }\end{array}$ & $\begin{array}{l}\text { ED } \\
\text { (in.) }\end{array}$ & $\begin{array}{c}\mathrm{M}_{\mathrm{p}} \\
\text { kip-ft }\end{array}$ & $\begin{array}{c}\mathrm{V}_{\mathrm{p}} \\
\text { kips }\end{array}$ & $\begin{array}{c}\mathrm{e}^{\prime} \\
\text { (in.) }\end{array}$ & $\mathrm{e}^{\prime} /\left(\mathrm{M}_{\mathrm{p}} / \mathrm{V}_{\mathrm{p}}\right)$ & $\theta=\frac{\gamma \times e}{L}$ \\
\hline 1 & 8.1 & 0 & 618 & 119 & 39.8125 & 0.64 & 0.030 \\
\hline 2 & 8.1 & 2 & 618 & 119 & 35.8125 & 0.57 & 0.029 \\
\hline 3 & 8.1 & 3 & 618 & 119 & 33.8125 & 0.54 & 0.026 \\
\hline 4 & 8.1 & 4 & 618 & 119 & 31.8125 & 0.51 & 0.024 \\
\hline 5 & 8.1 & 5 & 618 & 119 & 29.8125 & 0.48 & 0.023 \\
\hline 6 & 8.1 & 6 & 618 & 119 & 27.8125 & 0.45 & 0.021 \\
\hline 7 & 8.1 & 7 & 618 & 119 & 25.8125 & 0.41 & 0.020 \\
\hline 8 & 8.1 & 8 & 618 & 119 & 23.8125 & 0.38 & 0.018 \\
\hline 9 & 8.1 & 9 & 618 & 119 & 21.8125 & 0.35 & 0.017 \\
\hline
\end{tabular}

The length of the link in T-EBF is equal to the opening length since the effective panel is located under the opening. The link rotation angle is limited by the story drift. In other words, EBF ductility demand is determined by the story drift. The inelastic frame drift angle $(\theta)$ can be estimated from the geometry based on link rotation, as listed in Table (5.1) for link rotation $\gamma=0.09 \mathrm{rad}$. As clearly seen in the results, increases in ED dimension reduces the frame drift angle. For instance, changing the ED parameter from zero to nine leads to a story drift reduction of $43 \%$ while increasing the link shear force by only about $6 \%$.

The frame base shear-displacement relationships are shown in Figure (5.3). As seen in Figure (5.3), the links with less end distance need to dissipate larger seismic energy for the same link rotation compared to links with larger ED. However, all links successfully reached almost double the target link rotation $(\gamma=0.15 \mathrm{rad})$ with no apparent link buckling. 


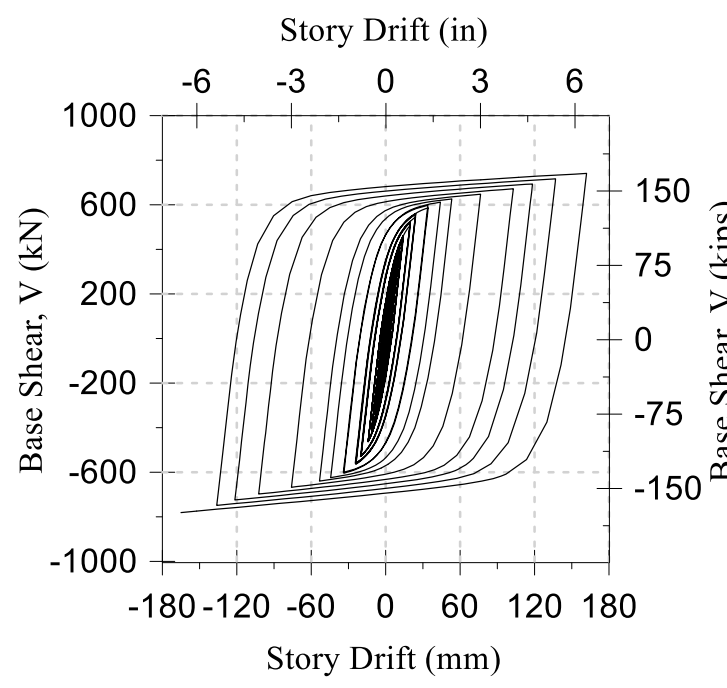

a) $\mathrm{ED}=0$

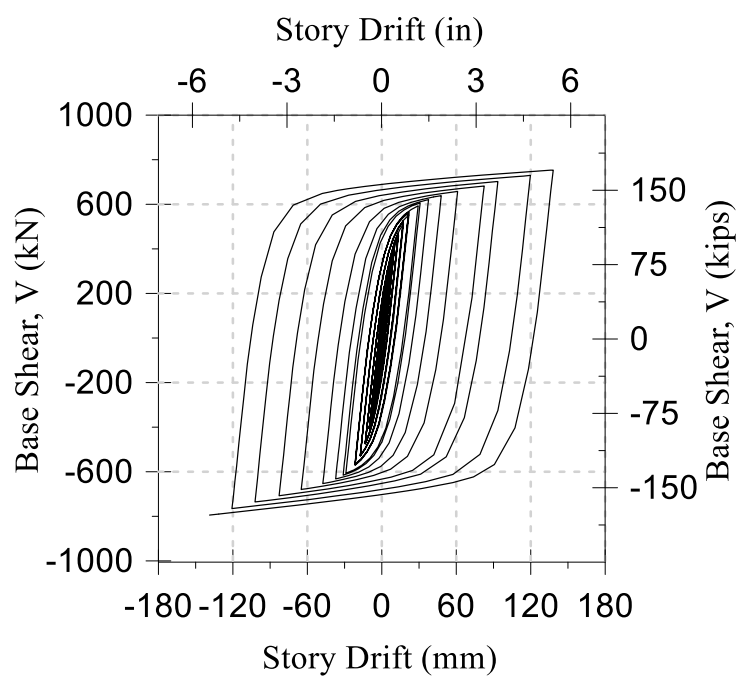

c) $\mathrm{ED}=4$

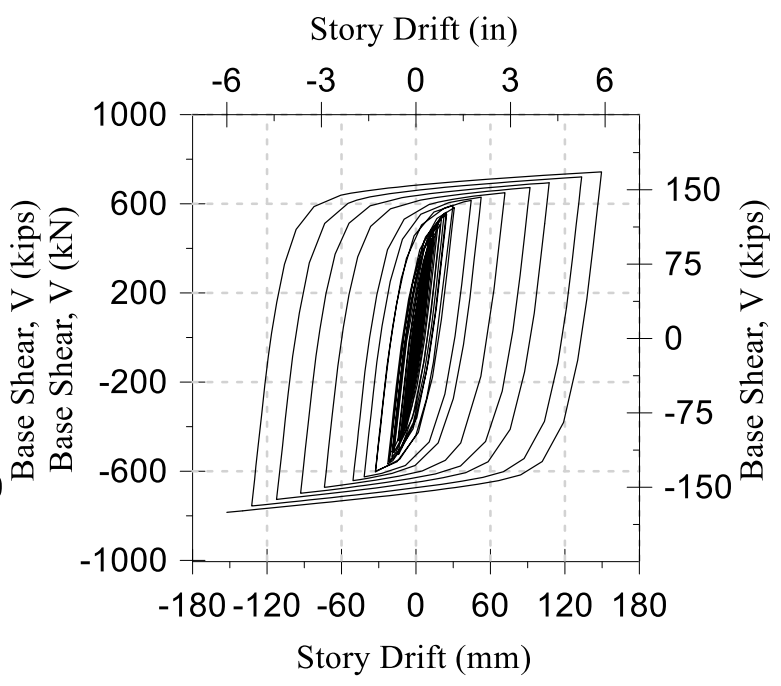

b) $\mathrm{ED}=2$

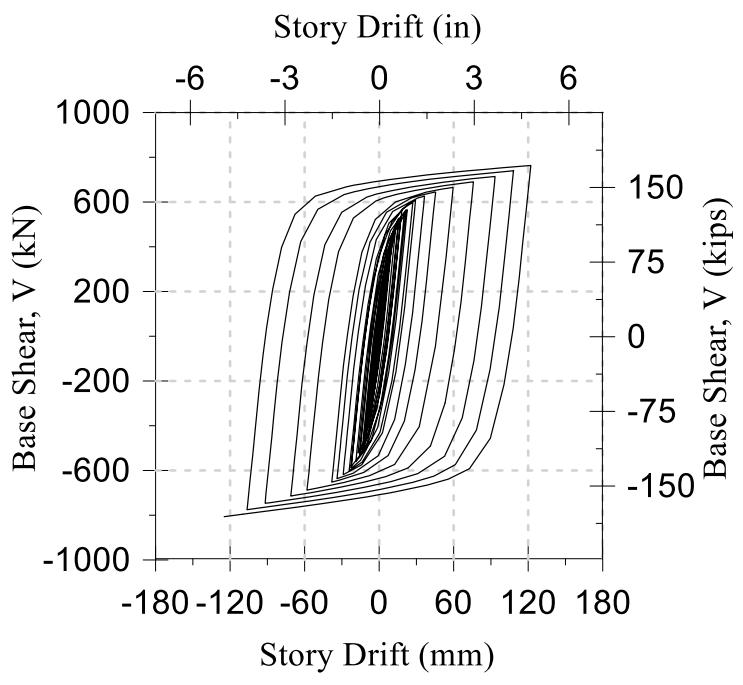

d) $\mathrm{ED}=6$ 


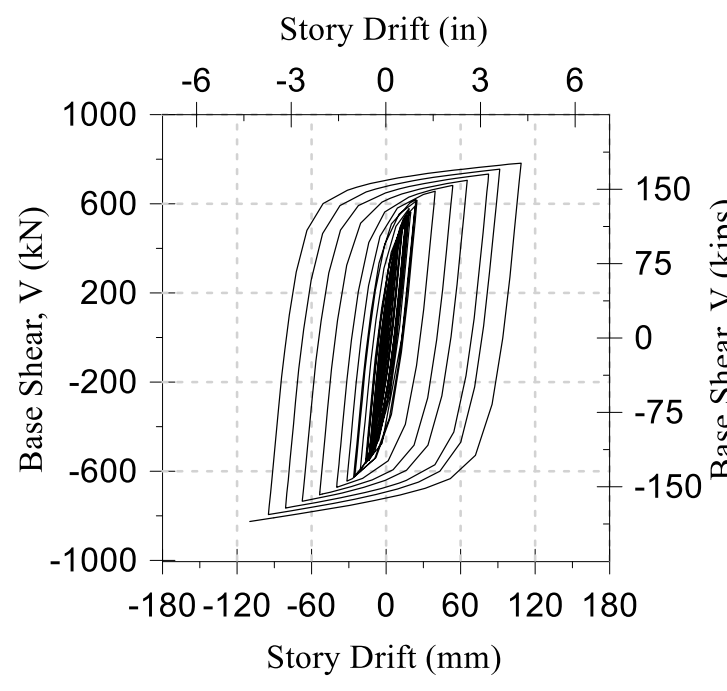

e) $\mathrm{ED}=8$

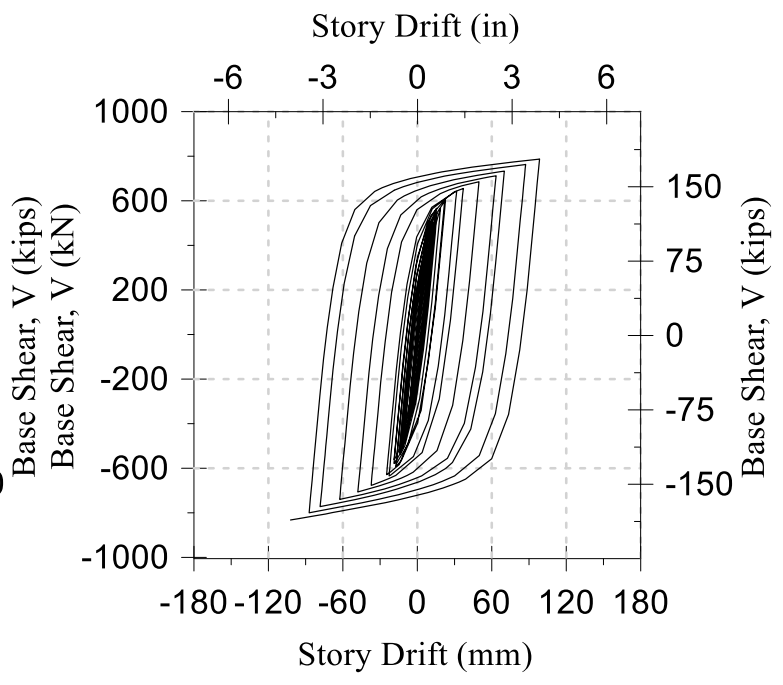

f) $\mathrm{ED}=9$

Figure 5.3 Effect of ED parameter on story drift

In order to estimate the effects of end distance on link behavior in T-EBF, hysteresis curves were plotted to represent the link rotation-shear link for the chosen values. There was no cyclic strength degradation for the models involved in this study, up to $0.15 \mathrm{rad}$. Obviously, the curves shown in Figure (5.4) display no influence from the end distance on link behavior except for a small increase in the link shear force. 


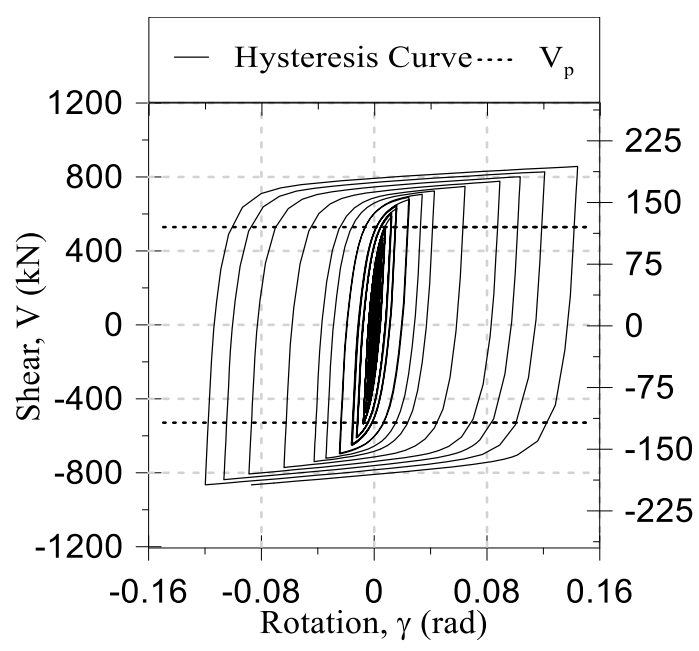

a) $\mathrm{ED}=0$

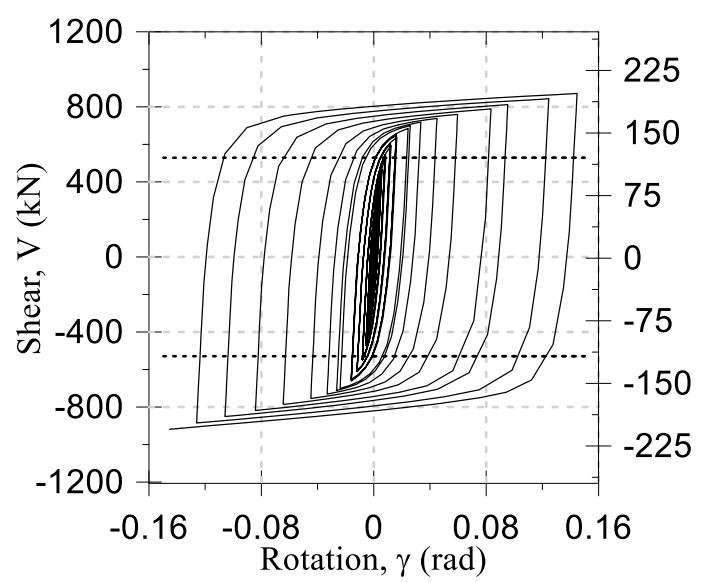

c) $\mathrm{ED}=4$

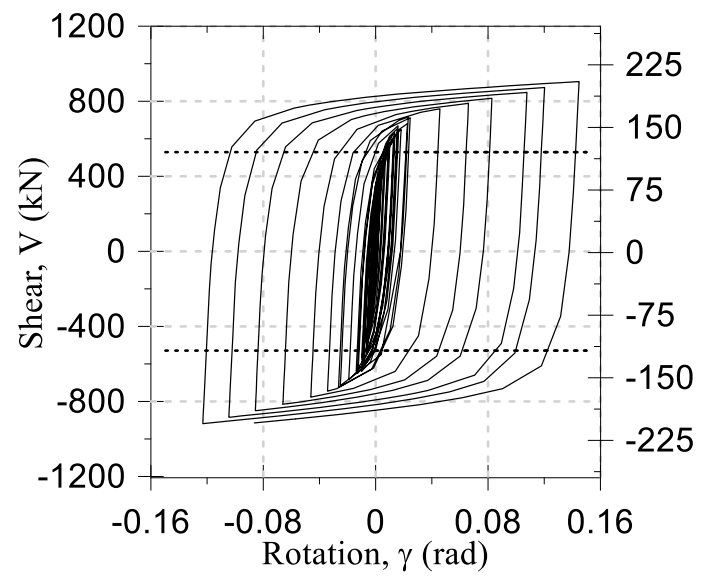

e) $\quad \mathrm{ED}=8$

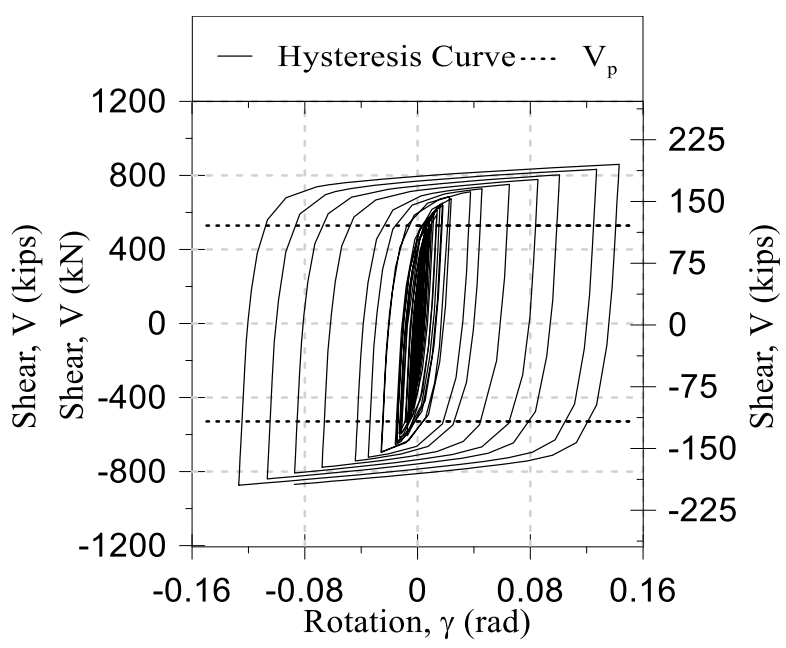

b) $\mathrm{ED}=2$

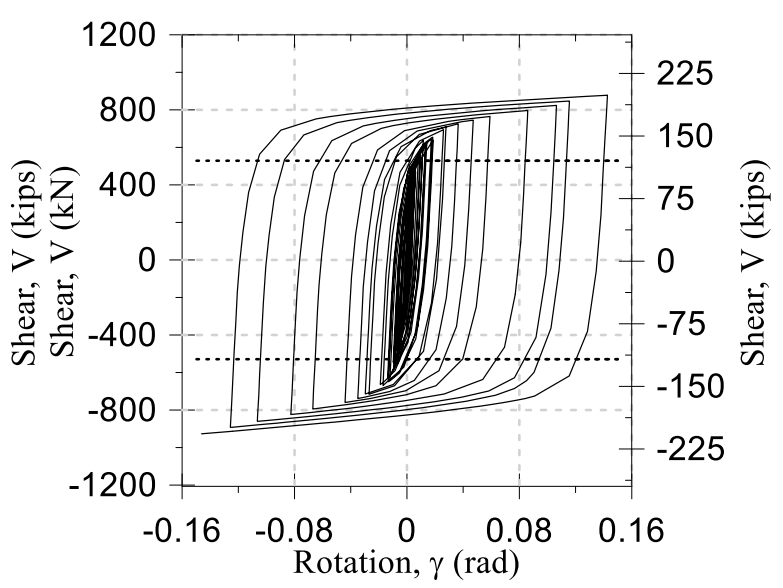

d) $\mathrm{ED}=6$

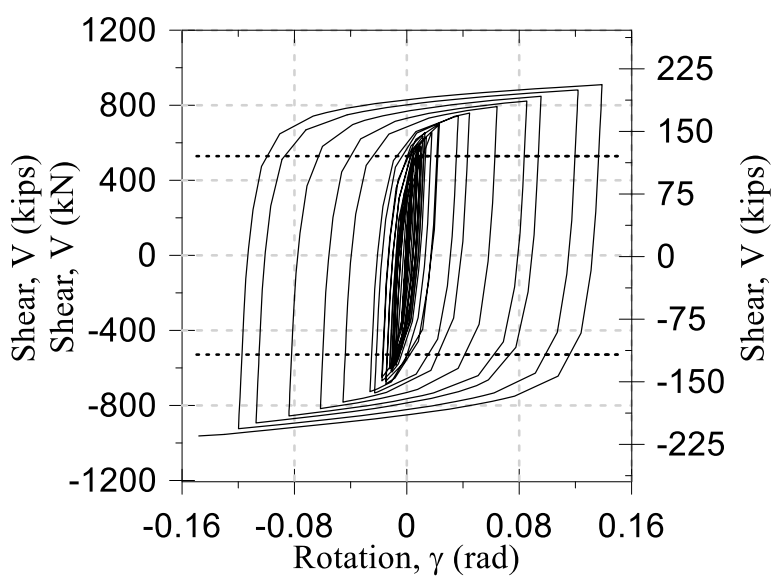

f) $\mathrm{ED}=9$

Figure 5.4 Hysteretic curves of the models 1 to 9 
Table (5.2) below shows the plastic strain distribution within the modeled links at link rotation angle $0.09 \mathrm{rad}$. As was clear from Chapter 2, the FEA can capture the buckling failure of the link in T-EBF and the Figures in Table (5.2) showed no buckling in any of the models studied in this section.

Plastic strain, PEEQ, was selected as the principles to judge the susceptibility of the fracture in the links. In general, fracture possibility is reduced as the PEEQ is decreased (Berman et al. 2009). The initial fracture of the link in EBF, which is constructed of seismic grade steel, is estimated to the value of $280 \%$ of plastic strain, PEEQ, based on studies that show relationships between the steel hardness, peak cycle plastic shear strain, and number of cycles (Ashikov et al., 2016). This value was compatible with FEA results in this research which was compared with the experimental tests shown in the previous chapter.

Plastic strain results at fifteen points of interest at link rotation angle 0.09 rad are listed in Table (5.3). The locations of the points are specified in Figure (5.5) and the values are represented at the link web and the top outer layer of the flanges. The web mid-point, indicated as the number 1 in Table (5.3), is one of the most significant points of interest as the energy dissipation of the shear link in EBF occurs within the web and depends on the shear strain, which has the maximum value at the effective panel web center. No significant influences of the ED parameter were found on the stain at point 1.

As expected, all the models have low PEEQ at the ends of the links and near the connections compared with PEEQ at the middle of the web. In general, by comparing the values in Table (5.3), the models with larger end distance, have a larger reduction in the 
plastic strain. For instance, point 8 , located at the middle flange near the endplate, has the largest PEEQ value recorded in the flanges. Results show that using higher ED distance can significantly reduce the value of point 8 . However, even without leaving a distance, $\mathrm{ED}=0$, it still small to cause potential damage or fractures. The points on the top flange located above the opening edge, points 5 and 6 , are the only points that increase with increasing the end distance due to the bent of the flange at these locations and larger displacement expected to occur compared with smaller ED. 
Table 5.2 PEEQ distributions of the models, ED models

\begin{tabular}{|c|c|c|c|c|}
\hline & & & PEEQ at $\gamma=0.09$ & PEEQ at $\gamma=0.15$ \\
\hline Model & ED & ho & 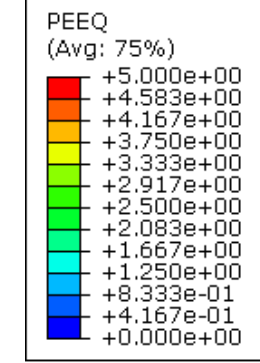 & 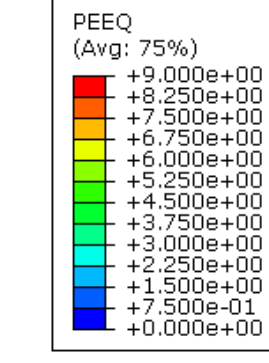 \\
\hline 1 & 0 & 7.5 & & \\
\hline 4 & 4 & 7.5 & & \\
\hline 6 & 6 & 7.5 & & \\
\hline 9 & 9 & 7.5 & & \\
\hline
\end{tabular}



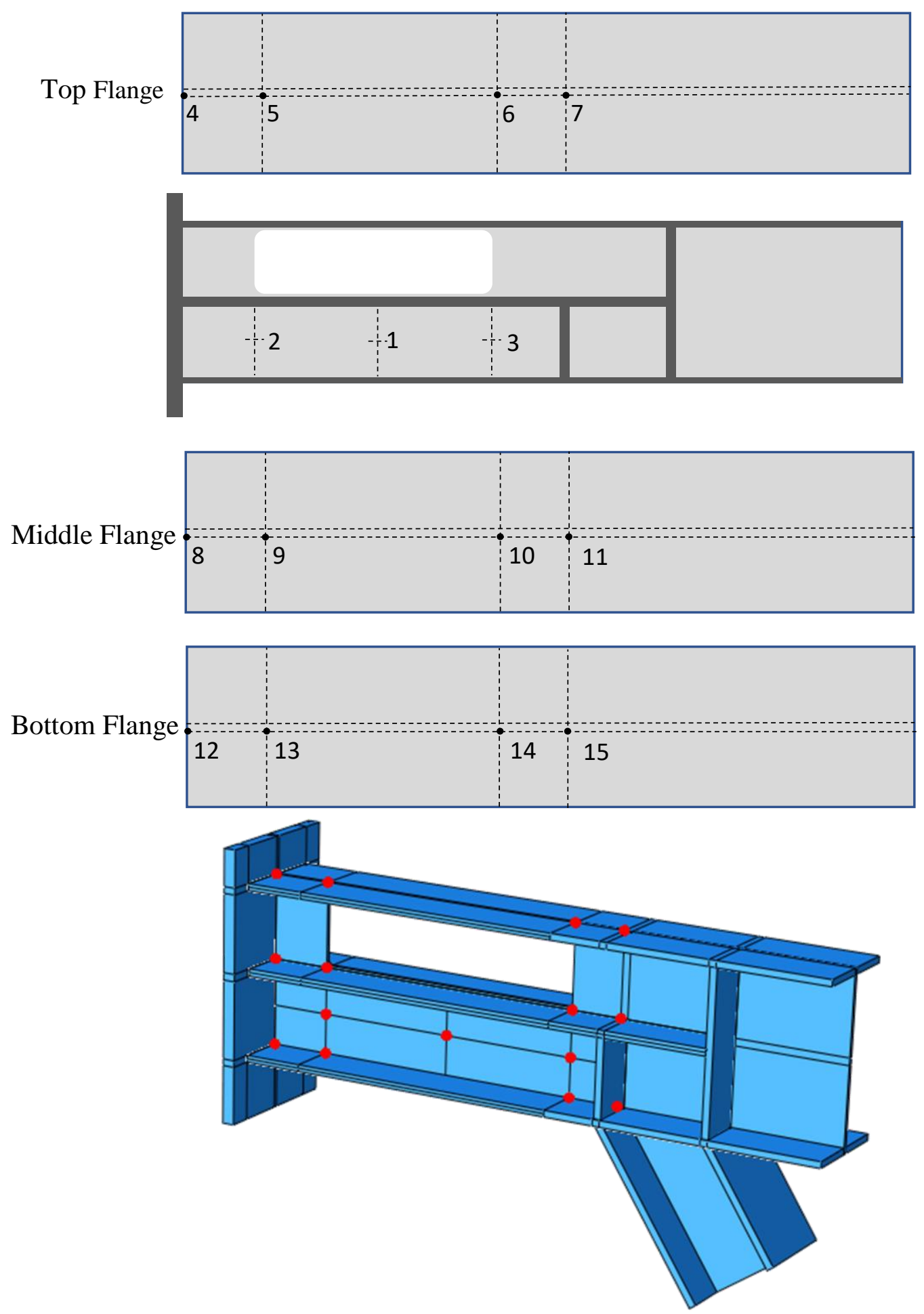

Figure 5.5 Points of interest within the link 
Table 5.3 PEEQ for various cases of ED under cyclic loading at $\gamma=0.09 \mathrm{rad}$

\begin{tabular}{lccc} 
& \multicolumn{3}{c}{ Web } \\
\cline { 2 - 4 } Models & \multicolumn{3}{c}{ Points } \\
\cline { 2 - 4 } & 1 & 2 & 3 \\
nDy & 0.953 & 0.3 & 0.181 \\
ED2 & 0.973 & 0.43 & 0.4 \\
ED4 & 0.982 & 0.51 & 0.48 \\
ED7 & 0.993 & 0.55 & 0.512 \\
ED9 & 0.998 & 0.61 & 0.57
\end{tabular}

\begin{tabular}{lcccc} 
Models & \multicolumn{5}{c}{ Top Flange } \\
\cline { 2 - 5 } & 4 & 5 & 6 & 7 \\
\cline { 2 - 5 } ED0 & 0.028 & - & - & 0.102 \\
ED2 & 0.026 & 0.054 & 0.089 & 0.001 \\
ED4 & 0.011 & 0.075 & 0.104 & 0.000 \\
ED7 & 0.0 & 0.108 & 0.135 & 0.000 \\
ED9 & 0.0 & 0.137 & 0.162 & 0.000
\end{tabular}

\begin{tabular}{lcccc} 
Models & \multicolumn{4}{c}{ Middle flange } \\
\cline { 2 - 5 } & 8 & 9 & 10 & 11 \\
\cline { 2 - 5 } ED0 & 0.44 & - & - & 0.22 \\
ED2 & 0.033 & 0.23 & 0.17 & 0.02 \\
ED4 & 0.0045 & 0.2 & 0.15 & 0.007 \\
ED7 & 0.0 & 0.16 & 0.13 & 0.005 \\
ED9 & 0 & 0.18 & 0.17 & 0.003
\end{tabular}

\begin{tabular}{lcccc} 
& \multicolumn{5}{c}{ Bottom Flange } \\
\cline { 2 - 5 } Models & 12 & \multicolumn{4}{c}{13} & 14 & 15 \\
\cline { 2 - 5 } ED0 & 0.087 & - & - & 0.076 \\
ED2 & 0.057 & 0.244 & 0.20 & 0.057 \\
ED4 & 0.029 & 0.23 & 0.18 & 0.038 \\
ED7 & 0.011 & 0.218 & 0.2 & 0.021 \\
ED9 & 0.006 & 0.23 & 0.22 & 0.017
\end{tabular}

For models 1 and 9, where ED's are equal to 0 and 9" respectively, local imperfections are taken into consideration and the hysteretic curves are plotted and compared with the models without imperfection. The results show that the effectiveness 
of the imperfection can be seen at very high rotation, after $\gamma=0.09 \mathrm{rad}$, as illustrated in Figure (5.6).
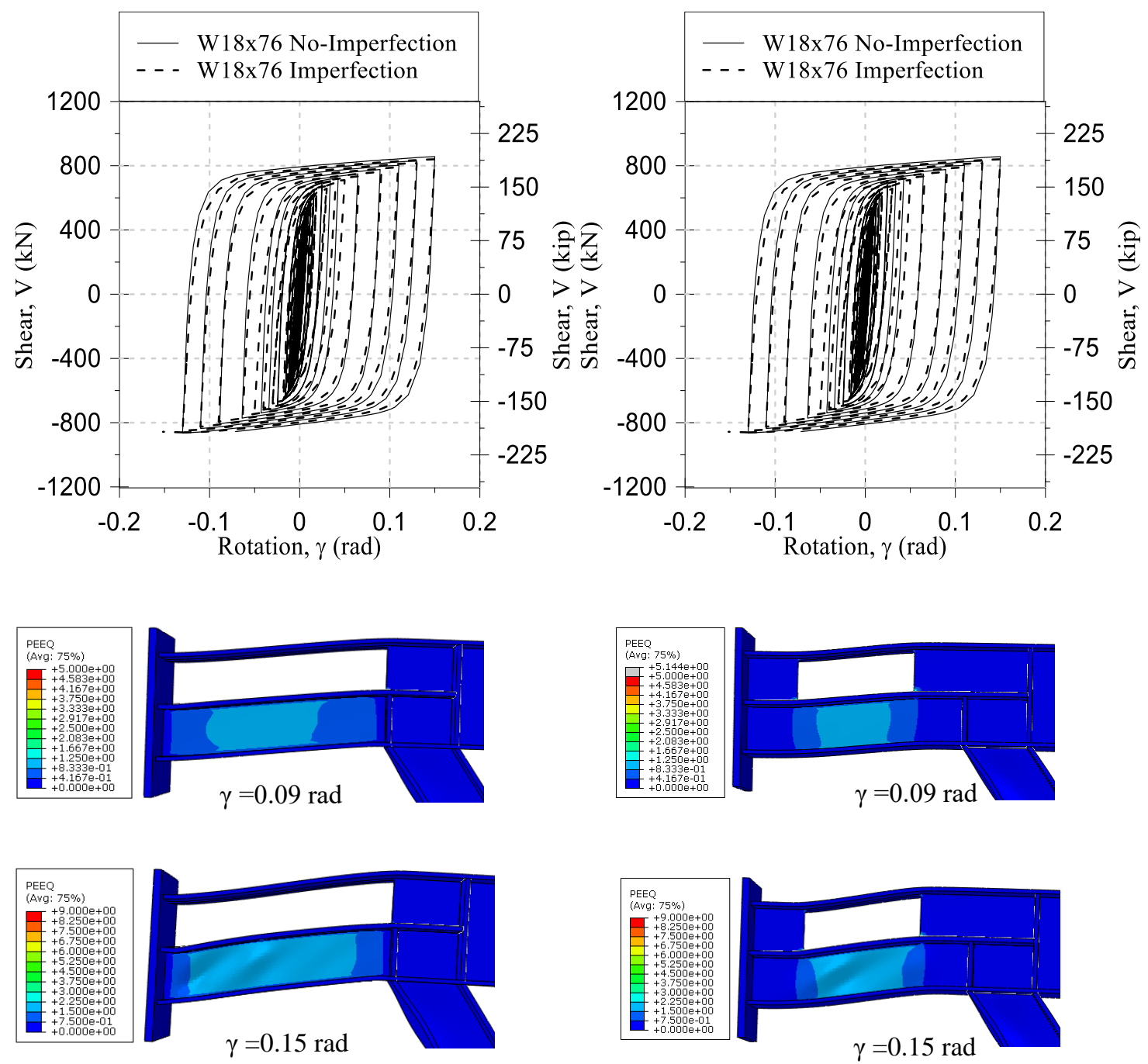
a) $\mathrm{ED}=$ Zero
b) $\mathrm{ED}=9 "$

Figure 5.6 Effect of initial imperfections on PEEQ and hysteresis of models 1 and 9 


\subsubsection{Effective Depth $\left(\mathrm{h}_{\mathrm{w}}\right)$}

One of the most important factors in T-EBF is the effective depth of the shear link $h_{w}$ as it is responsible for the shear strength of the link. Therefore, models 10 to 15 shown in Table (5.4) were used to investigate the effectiveness of changing the parameter $h_{w}$ on the cyclic behavior of the T-EBF. In this study, the opening depth was increased from 4" to $12^{\prime \prime}$, with $2^{\prime \prime}$ increments.

Table 5.4 hw Parameter models, dimensions in inches

\begin{tabular}{|c|c|c|c|c|c|c|}
\hline & $\mathrm{h}_{\mathrm{w}}$ & $\mathrm{e}_{\mathrm{o}}$ & $\mathrm{M}_{\mathrm{p}}$ & $\mathrm{V}_{\mathrm{p}}$ & $\mathrm{e}^{\prime}$ & $\mathrm{e}^{\prime} /\left(\mathrm{M}_{\mathrm{p}} / \mathrm{V}_{\mathrm{p}}\right)$ \\
\hline 10 & 11.6 & 6.17 & 679 & 164 & 27.81 & 0.56 \\
\hline 11 & 10.6 & 5.67 & 658 & 151 & 27.81 & 0.53 \\
\hline 12 & 9.6 & 5.17 & 642 & 138 & 27.81 & 0.50 \\
\hline 13 & 7.6 & 4.17 & 611 & 113 & 27.81 & 0.43 \\
\hline 14 & 5.6 & 3.17 & 588 & 87 & 27.81 & 0.34 \\
\hline 15 & 3.6 & 2.17 & 571 & 62 & 27.81 & 0.25 \\
\hline
\end{tabular}

Table (5.4) displays the main dimensions and plastic strength of the models used in this part. The link length, as well as ED parameter, are equal for all models used here. End distance was set at 6", the same as that used in the experimental test.

From the base shear-story drift hysteresis curves shown in Figure (5.7), one can conclude that section depth can impact curve behavior by reducing the base shear with the same story drift. This reduces the base shear by about $53 \%$ with the same story drift when $h_{w}$ is reduced from $11.6^{\prime \prime}$ to $3.6^{\prime \prime}$. The story drift angle is the same for the model involved in this analysis, which is about $0.02 \mathrm{rad}$, at link rotation of $0.09 \mathrm{rad}$ 

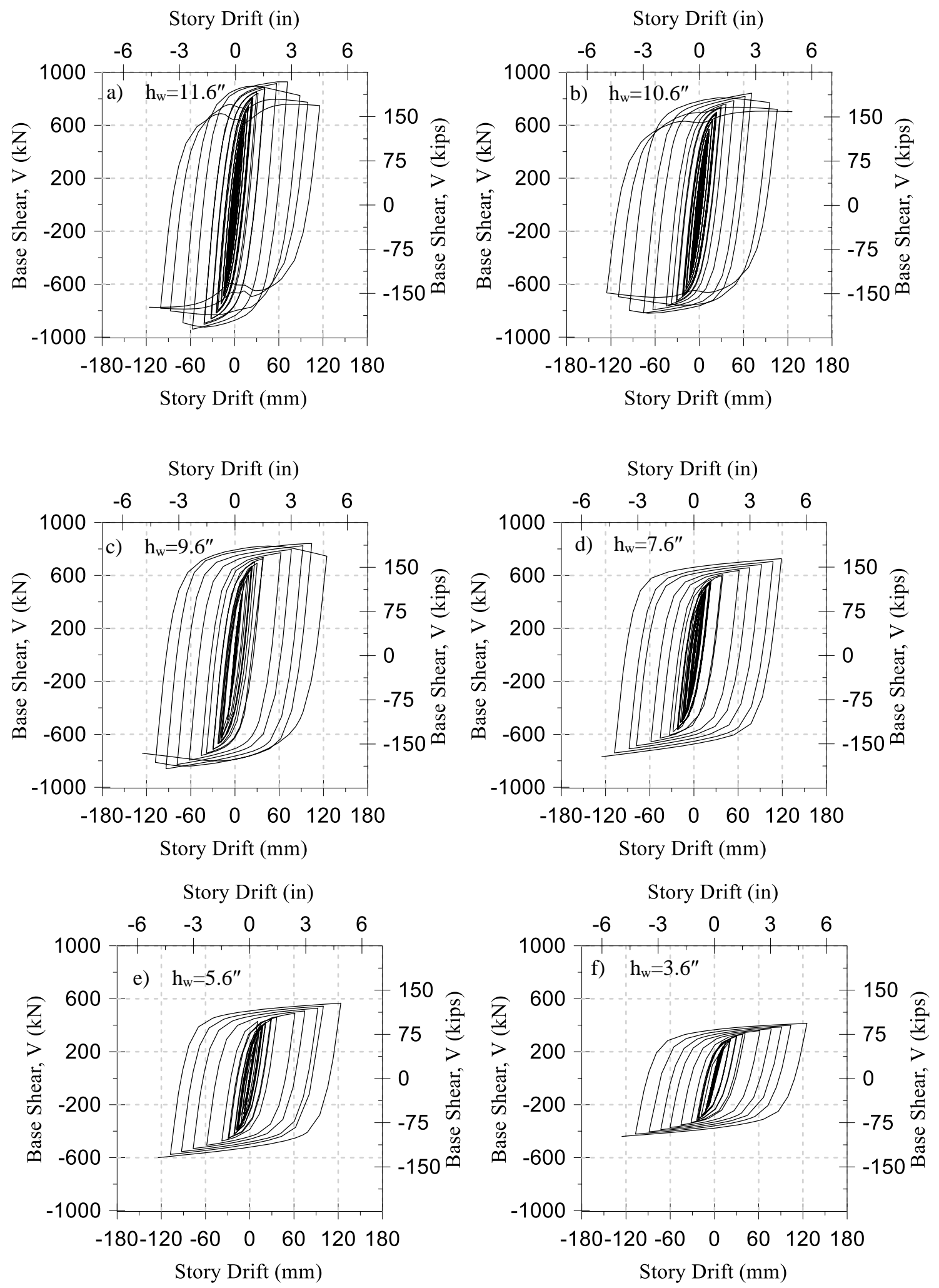

Figure 5.7 Effect of hw parameter on story drift 
Figure (5.8) illustrates the link- rotation hysteresis behavior to investigate the effect of the selected parameter on the link in T-EBF. The study includes looking at any type of buckling or strength degradation. The results show that for links with small opening depth, $\mathrm{h}_{\mathrm{o}}=4$ ", strength degradation occurred before reaching the desired target link rotation, $0.08 \mathrm{rad}$, and continue to decrease the shear strength until about $80 \%$ at link rotation, $0.15 \mathrm{rad}$. Increasing the opening depth is shown to improve the link cyclic behavior.

The plastic strain distribution within the T-EBF link is plotted for the selected modeled links in Table (5.5) which also shows possible link web buckling. The web buckling is responsible for the strength degradation that was seen in the shear force-link rotation hysteresis plots. As shown in Table (5.5), links with higher effective depth might suffer from sever web buckling. The figure also shows that shearing strain happens within the link web which is preferable in EBF design.

Table (5.6) demonstrates that the plastic strain for the chosen points within the link in T-EBF exhibits slight changes in the value of PEEQ at the target link rotation. The web center has the highest value within the link. Increasing the opening depth has a limited effect on PEEQ values when increasing the opening depth. The $h_{w}$ has also influence on reducing strain near the link ends at the column and brace connection when it decreases from $11.6^{\prime \prime}$ to $3.6^{\prime \prime}$. 


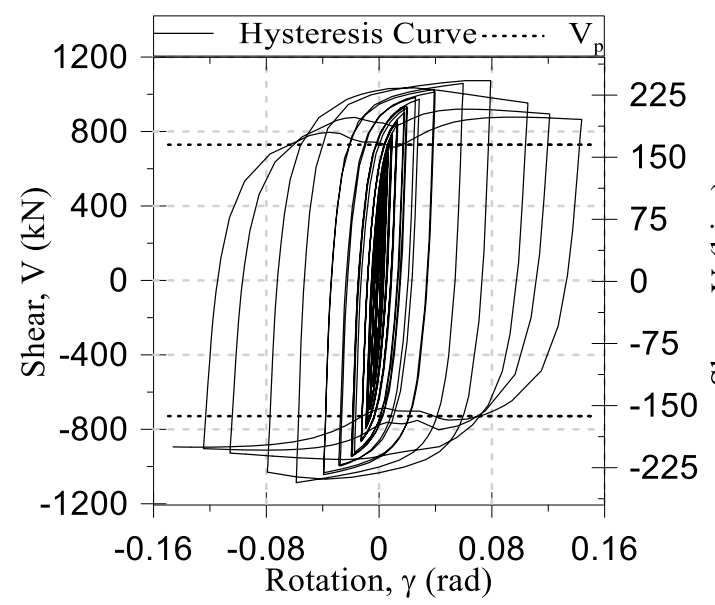

a) $h_{w}=11.6$

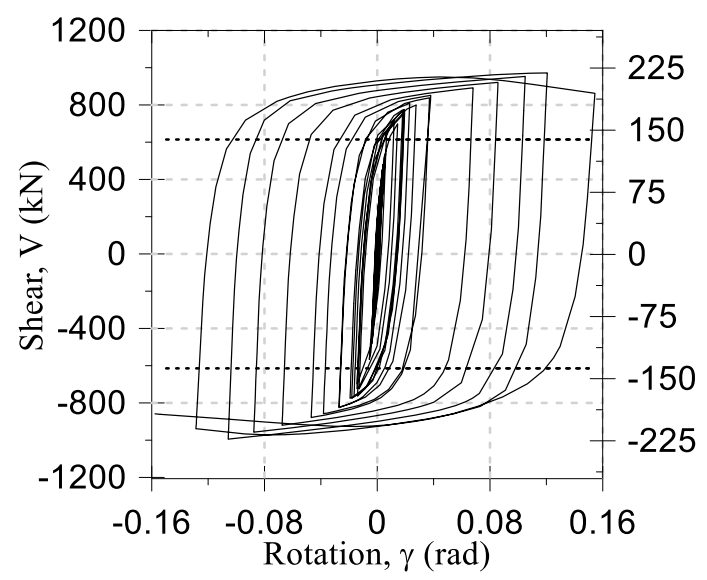

c) $h_{w}=9.6$

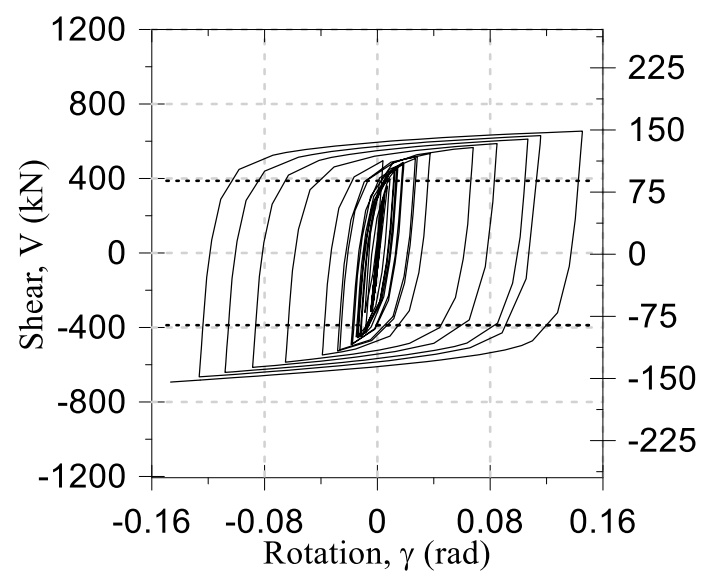

e) $\mathrm{h}_{\mathrm{w}}=5.6$

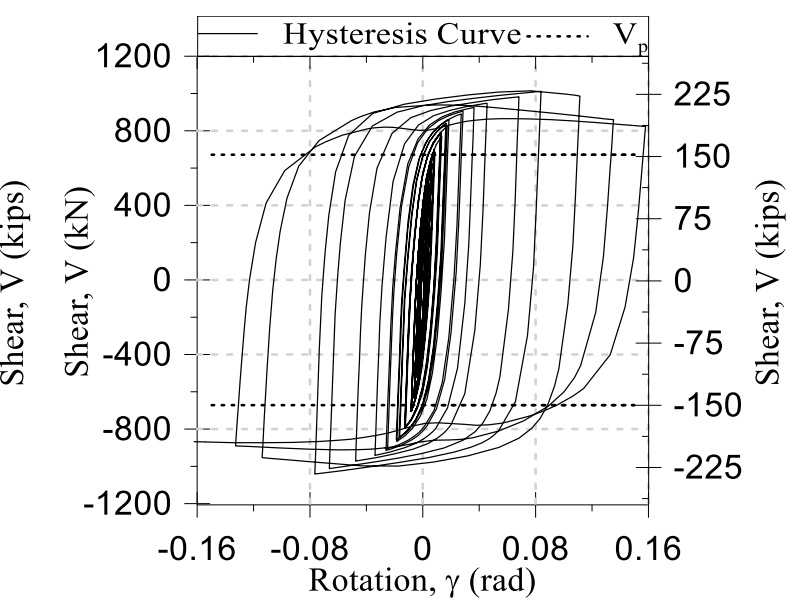

b) $h_{w}=10.6$

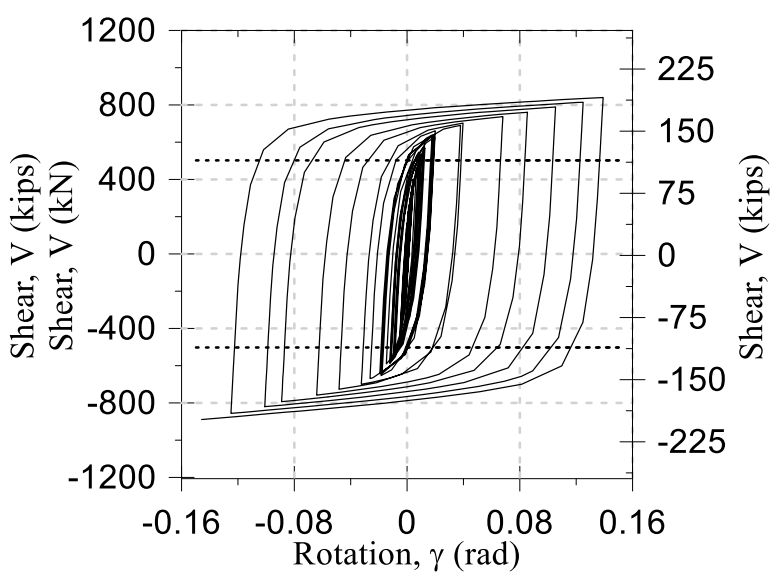

d) $h_{w}=7.6$

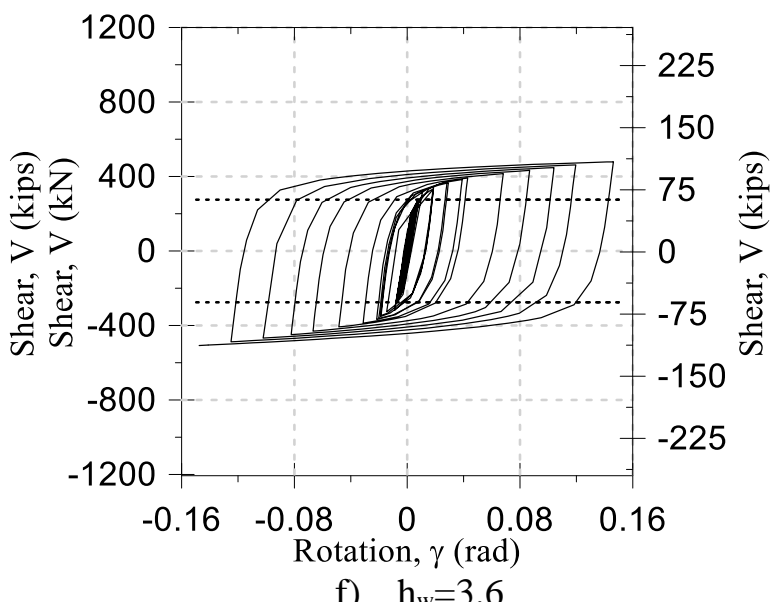

f) $h_{w}=3.6$

Figure 5.8 Hysteretic curves of the models 10 to 15 
Table 5.5 Distribution of PEEQ within the links

\begin{tabular}{|c|c|c|c|c|}
\hline & & & PEEQ at $\gamma=0.09 \mathrm{ra}$ & PEEQ at $\gamma=0.15 \mathrm{rad}$ \\
\hline Model & ED & $\mathbf{h}_{\mathbf{w}}$ & 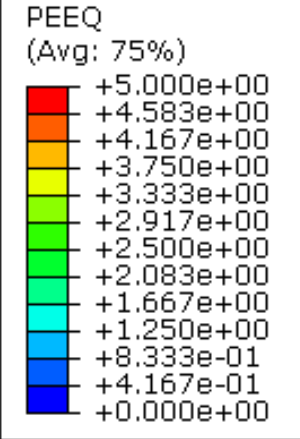 & 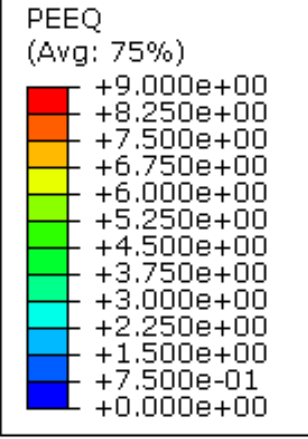 \\
\hline 10 & 6 & 11.6 & & \\
\hline 12 & 6 & 9.6 & & \\
\hline 14 & 6 & 5.6 & & \\
\hline 15 & 6 & 3.6 & & \\
\hline
\end{tabular}


Table 5.6 PEEQ at $\gamma=0.09 \mathrm{rad}$

\begin{tabular}{lccc} 
& \multicolumn{3}{c}{ Web } \\
\cline { 2 - 4 } Models & \multicolumn{3}{c}{ Points } \\
\cline { 2 - 4 } & 1 & 2 & 3 \\
10 & 1.027 & 0.6 & 0.511 \\
12 & 0.988 & 0.58 & 0.485 \\
13 & 0.944 & 0.487 & 0.467 \\
15 & 0.855 & 0.43 & 0.421
\end{tabular}

\begin{tabular}{lcccc} 
Models & \multicolumn{5}{c}{ Top Flange } \\
\cline { 2 - 5 } & 4 & 5 & 6 & 7 \\
\cline { 2 - 5 } 10 & 0.059 & 0.071 & 0.099 & 0.001 \\
12 & 0.009 & 0.100 & 0.129 & 0.000 \\
13 & 0.002 & 0.103 & 0.131 & 0.000 \\
15 & 0.0 & 0.125 & 0.138 & 0.000
\end{tabular}

\begin{tabular}{ccccc} 
Models & \multicolumn{5}{c}{ Middle flange } \\
\cline { 2 - 5 } & 8 & 9 & 10 & 11 \\
\cline { 2 - 5 } 10 & 0.006 & 0.166 & 0.13 & 0.006 \\
12 & 0.0 & 0.168 & 0.139 & 0.005 \\
13 & 0.0 & 0.17 & 0.143 & 0.0 \\
15 & 0.0 & 0.17 & 0.148 & 0.0
\end{tabular}

\begin{tabular}{lcccc} 
& \multicolumn{5}{c}{ Bottom Flange } \\
\cline { 2 - 5 } Models & 12 & \multicolumn{1}{c}{13} & 14 & 15 \\
\cline { 2 - 5 } 10 & 0.025 & 0.228 & 0.20 & 0.07 \\
12 & 0.018 & 0.231 & 0.21 & 0.042 \\
13 & 0.011 & 0.24 & 0.22 & 0.035 \\
15 & 0.009 & 0.31 & 0.28 & 0.00
\end{tabular}

To study the sensitivity of the imperfections on the behavior of T-EBF models, two models with the biggest effective depth, hw, are modeled with initial imperfections and compared with the models without any imperfections. The model 10, with $h_{w}=11.6 "$, has a slight drop in shear strength, about $4 \%$ at the rotation $0.09 \mathrm{rad}$, compared with 
models without initial buckling and continuing about the same rate until the end of the analysis. Decreasing in shear strength was also observed in model 11, which was approximately $5 \%$ at $\gamma=0.09$ rad. Results from imperfection analysis show that T-EBF has a little sensitivity to initial buckling beyond the target rotation $0.08 \mathrm{rad}$, while no clear effect before the target rotation.
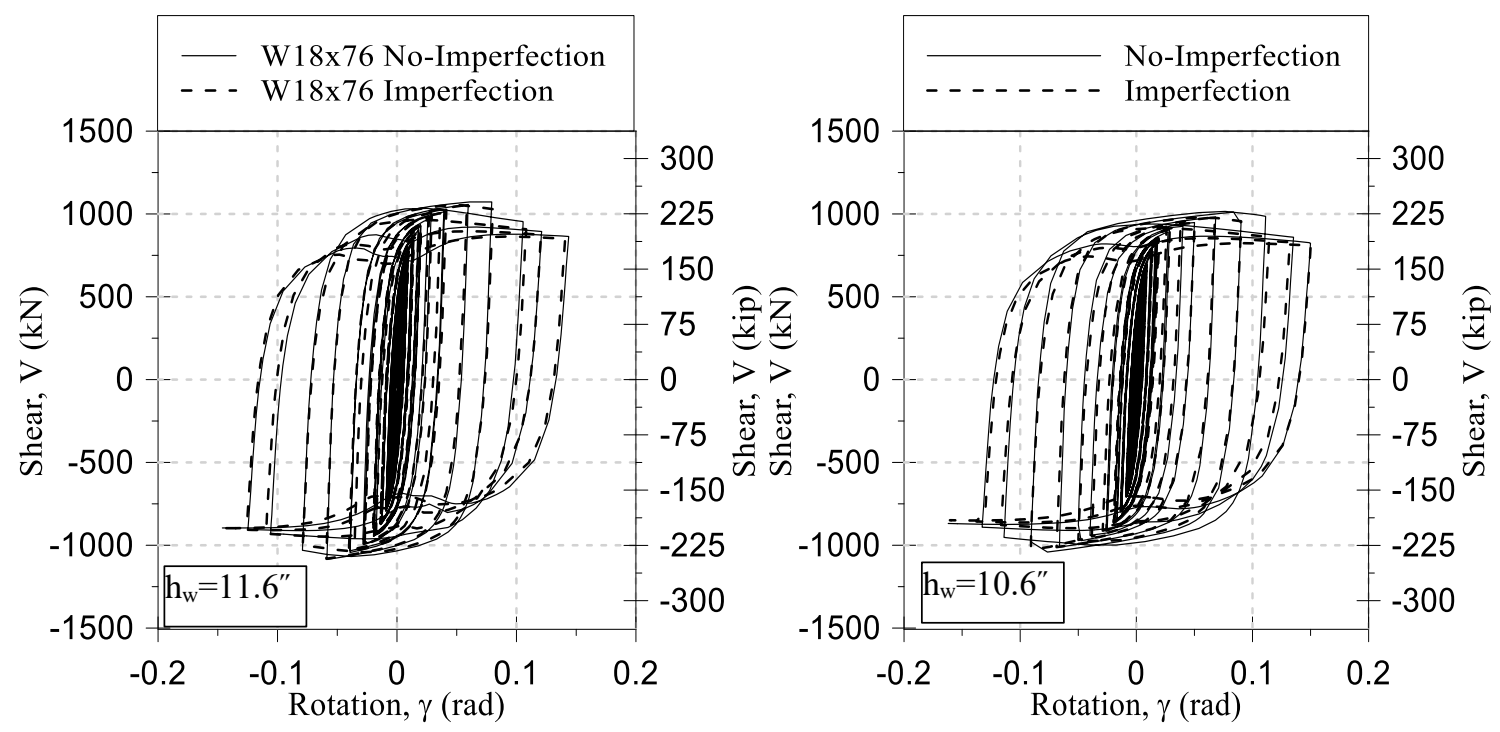

a) Base shear versus link rotation
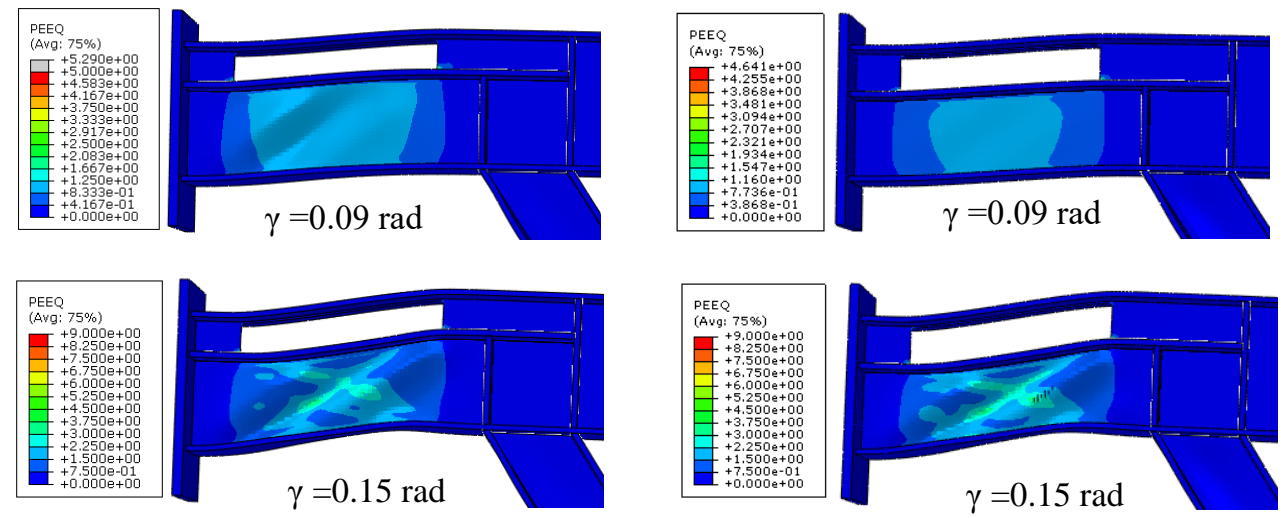

b) Model 10 with imperf.

c) Model 11 with imperfection

Figure 5.9 Effective of initial imperfection, models 10 and 11 


\subsubsection{Horizontal Stiffeners Thickness $t_{\mathrm{hs}}$}

The horizontal web stiffener, which acts as a top flange in the T-EBF link, has the potential to improve link behavior and should be designed for the developed forces in the link. In this part, three models are used to detect the impact of changing the horizontal thickness $\left(\mathrm{t}_{\mathrm{hs}}\right.$ ) on link behavior. The stiffener thickness used in the models are $0.25^{\prime \prime}, 0.5^{\prime \prime}$, and $0.75^{\prime \prime}$. The results show that using insufficient thickness can lead to severe damages in the link. The buckling of the flanges in EBF links can result in link fractures due to high strains. It also can lead to web buckling as well as lateral torsional buckling, resulting in strength degradation and limited link ductility. These types of failure were observed in the $0.25^{\prime \prime}$ model. Figure (5.10) illustrated the link rotation-shear force plots for the three models. In addition, Figure (5.11) displays the link behavior showing the buckling of the horizontal web stiffener for the 0.25 " stiffener model.

The AISC seismic provision requires section selection with flange compactness for moderately ductile members. Table (5.7) shows the flange compactness of the threestiffener thickness used in this part, as compared with AISC limitations. The results illustrated minimum flange thickness, or horizontal stiffener thickness must be $0.75^{\prime \prime}$.

Table 5.7 Flange compactness limitation

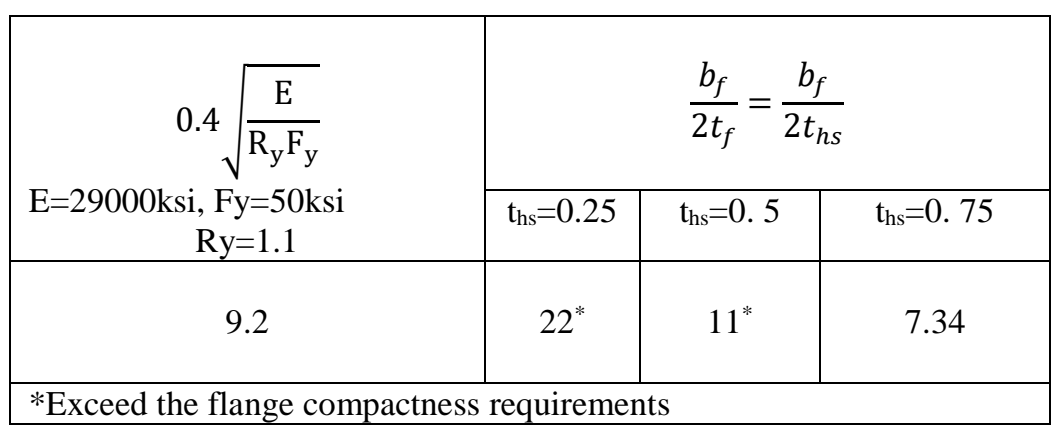



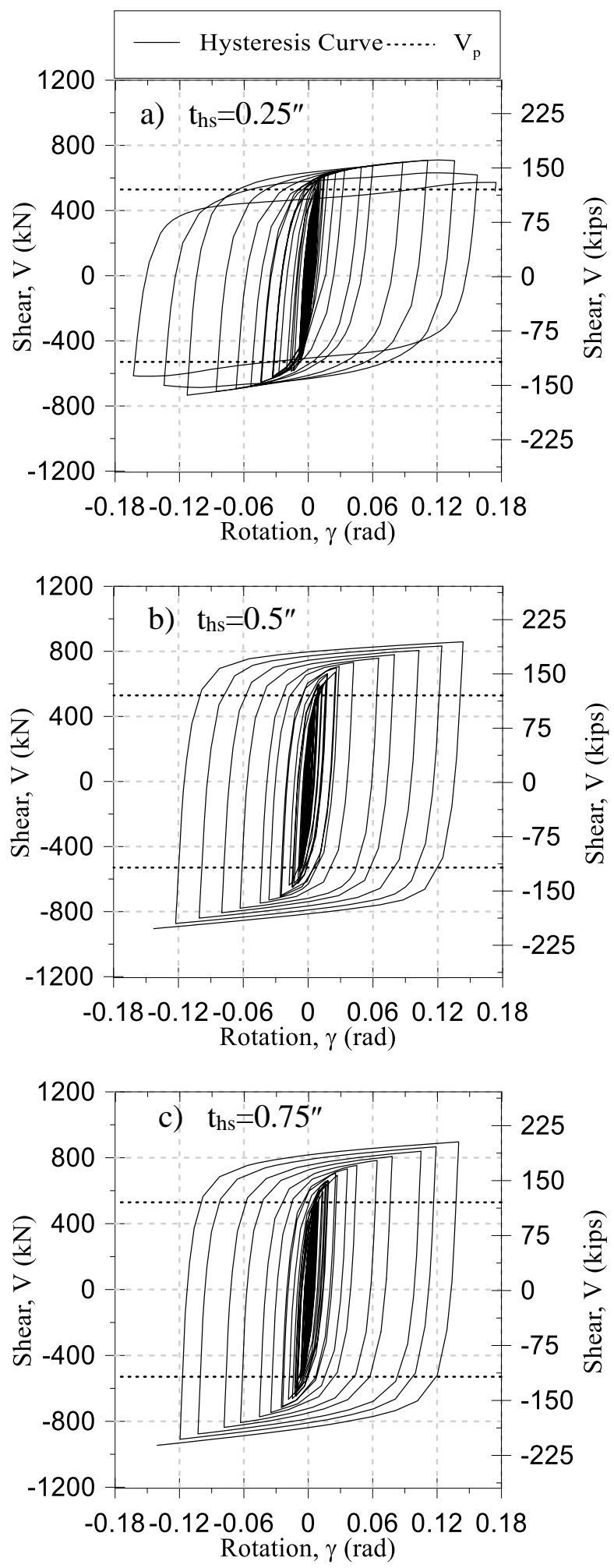

Figure 5.10 Hysteretic curves of the stiffeners thickness models 126 

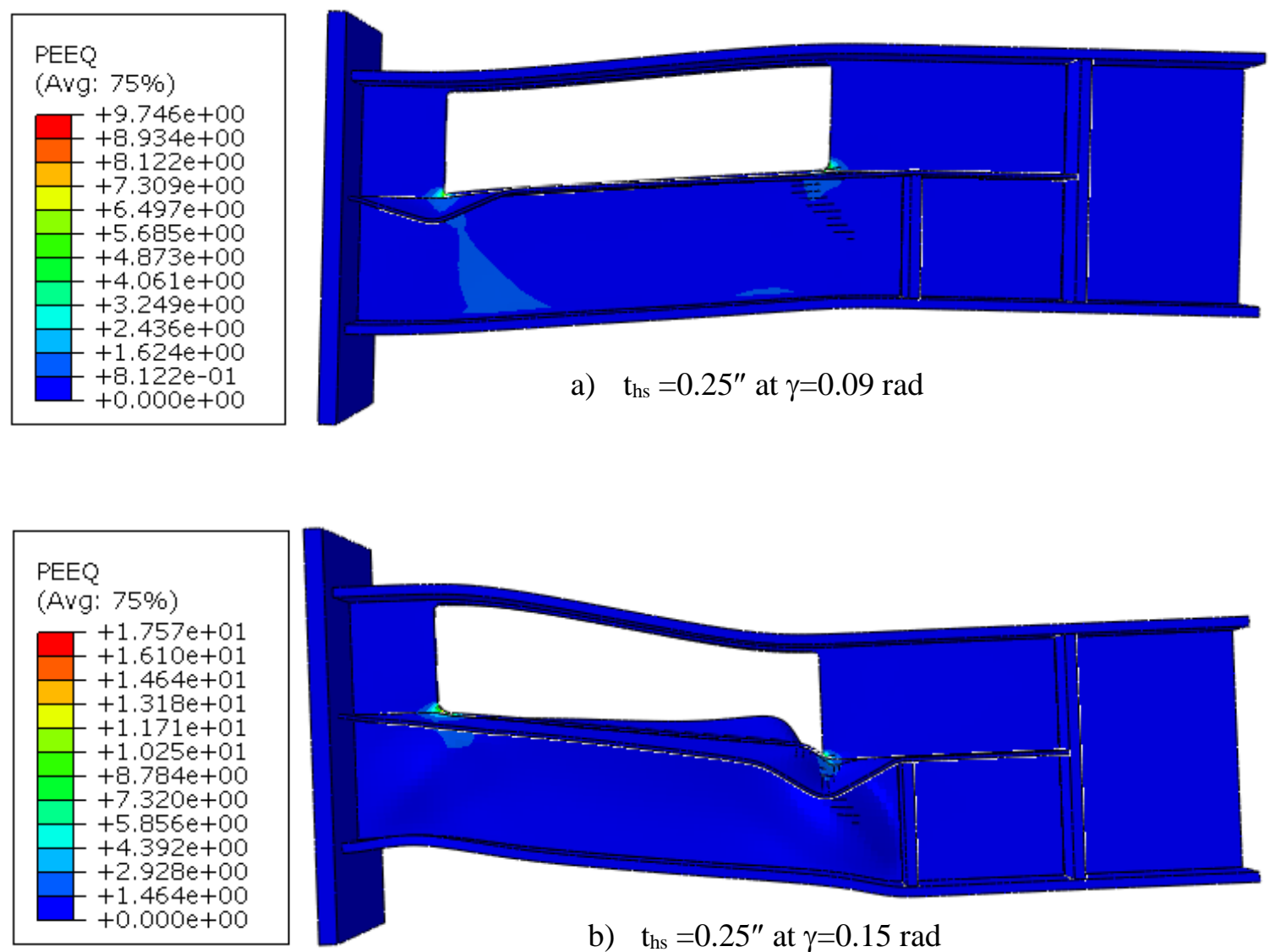

Figure 5.11 Link failure

\subsubsection{Transition Zone Radius}

An analytical study was carried out to control the effect of the fillet radius on the cracks that appeared in the opening corners during the experimental work. Reducing plastic strain can prevent the undesirable cracks. Table (5.8) contains the suggested fillet radius versus the cumulative plastic strain in the corner radius at a link rotation of 0.09 rad. FEA results have shown that the plastic strain at the transition zone decreases with an increase of the fillet radius. 
In addition, cases 5 and 6 in Table (5.8) were studied in more details to investigate the effects of the opening edge angle, shown in Figure (5.12), on the equivalent plastic strain within the web and flanges of the link. The two cases have the same fillet radius which is 3". Case no. 5 (denoted as R3) was modeled with a web opening edge at $90^{\circ}$ angle, while case no. 6 (R3_45) was modeled with an incline angle of opening edge equal to $45^{\circ}$. The results in Tables (5.8) and (5.9) show that the opening configuration with $45^{\circ}$ not only reduces the plastic strain in the radii area but also reduces PEEQ in the flanges and the web with maximum decreases in the middle web by about $11 \%$. The area outside the $45^{\circ}$ edge is the area of high-stress concentration and by removing the material of this area the stresses concentration can be decreased significantly.

According to the FEA results and verified through the experimental work, as described in previous chapters, it can be concluded that changing the opening geometry can reduce the possibility of corner cracks and improve link performance. 
Table 5.8 PEEQ at transition zone radius (at rotation $0.09 \mathrm{rad}$ )

\begin{tabular}{|c|c|c|c|c|}
\hline Case's No. & Model & $\begin{array}{l}\text { Radius } \\
\text { (in.) }\end{array}$ & $\begin{array}{c}\text { Edge angle } \\
\text { (degree) }\end{array}$ & $\begin{array}{c}\text { PEEQ at } \\
\text { radii }\end{array}$ \\
\hline 1 & & 0.5 & 90 & 4.88 \\
\hline 2 & & 1 & 90 & \\
\hline 3 & & 2 & 90 & 1.71 \\
\hline 4 & & 2 & 45 & 1.55 \\
\hline 5 & & 3 & 90 & 1.51 \\
\hline 6 & & 3 & 45 & 1.30 \\
\hline
\end{tabular}
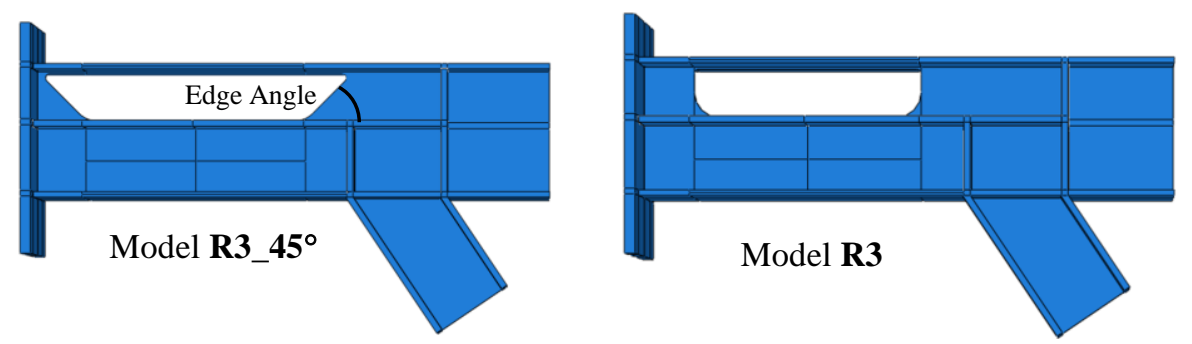

Figure 5.12 Two models to study the effect of opening edge on link performance 
Table 5.9 PEEQ results for both cases R3 and R3_45 at $\gamma=0.09 \mathrm{rad}$

\begin{tabular}{cccc} 
& \multicolumn{3}{c}{ Web } \\
\cline { 2 - 4 } Models & \multicolumn{3}{c}{ Points } \\
\cline { 2 - 4 } R3 & 1 & 2 & 3 \\
\cline { 2 - 4 } & 1.2 & 0.58 & 0.604 \\
R3_45 & 1.07 & 0.55 & 0.54
\end{tabular}

\begin{tabular}{ccccc} 
& \multicolumn{5}{c}{ Top Flange } \\
\cline { 2 - 5 } Models & 4 & 5 & 6 & 7 \\
\cline { 2 - 5 } R3 & 0.022 & 0.07 & 0.091 & 0.0012 \\
R3_45 & 0.004 & 0.004 & 0.007 & 0.0028
\end{tabular}

\begin{tabular}{ccccc} 
& \multicolumn{5}{c}{ Middle flange } \\
\cline { 2 - 5 } Models & 8 & 9 & 10 & 11 \\
\cline { 2 - 5 } R3 & 0.003 & 0.091 & 0.08 & 0.0086 \\
R3_45 & 0.008 & 0.08 & 0.054 & 0.009
\end{tabular}

\begin{tabular}{ccccc} 
& \multicolumn{5}{c}{ Bottom Flange } \\
\cline { 2 - 5 } Models & 12 & 13 & 14 & 15 \\
\cline { 2 - 5 } R3 & 0.23 & 0.124 & 0.073 & 0.26
\end{tabular}

$\begin{array}{lllll}\text { R3_45 } & 0.21 & 0.10 & 0.058 & 0.2\end{array}$

\subsubsection{Intermediate stiffeners}

Intermediate web stiffeners can be used to delay the web buckling in the link. The web buckling causes gradual deterioration and strength degradation. AISC seismic provisions (ANSI/AISC 341-16, 2016) require the addition of intermediate stiffeners in the link web using the below expressions;

For shear links $(\gamma=0.08 \mathrm{rad})$

$S \leq 30 t_{w}-\frac{d}{5}$ 
Intermediate web stiffeners must be full depth and on both sides when the link depth is equal or larger than 25", and only on one side of the link web when the depth is less than $25^{\prime \prime}$.

The stiffener size is also limited by the provision as shown in equations (5.2,5.3).

Stiffeners thickness $\leq t_{w}$ or $3 / 8$ whichever isl arger

Stiffeners width $\geq\left(\frac{b_{f}}{2}\right)-t_{w}$

In this part of analysis, three shear link models with different lengths and effective depth but with same ratio of $\left[\mathrm{e}^{\prime} /\left(\mathrm{M}_{\mathrm{p}} / \mathrm{V}_{\mathrm{p}}\right)\right]$, which is 0.56 , were investigated by adding intermediate web stiffeners, as listed in Table (5.10):

Intermediate web stiffeners spaced at intervals not exceeding $s=30 t_{w}-\frac{d}{5}=$ $30(0.425)-\frac{11.6}{5}=10.3$ for effective depth 11.6 .

Table 5.10 Models properties

\begin{tabular}{|c|c|c|c|c|c|c|c|}
\hline & & & & \\
\hline
\end{tabular}

Stability of the link against web buckling and lateral torsional buckling may increase by adding intermediate stiffeners as per the AISC seismic provision limitations. Results from analysis models 16 to 18 listed in Table (5.10) demonstrated that all links were able to reach the desired rotation of $0.08 \mathrm{rad}$. Slight web local buckling was 
observed at very large link rotation for model 16. Figure (5.13) describes the hysteresis curves of link rotation-shear forces and base shear-story drift of the shear link models.

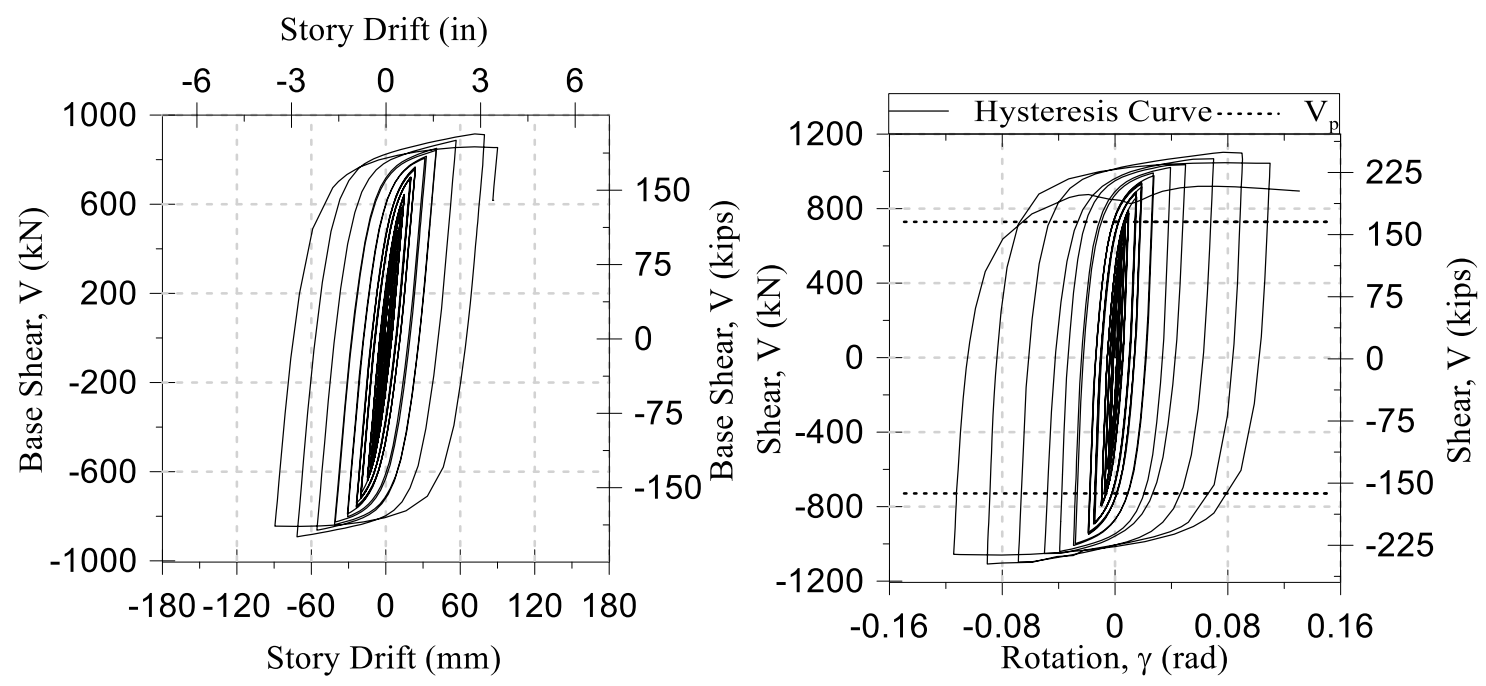

a) Shear link model 16

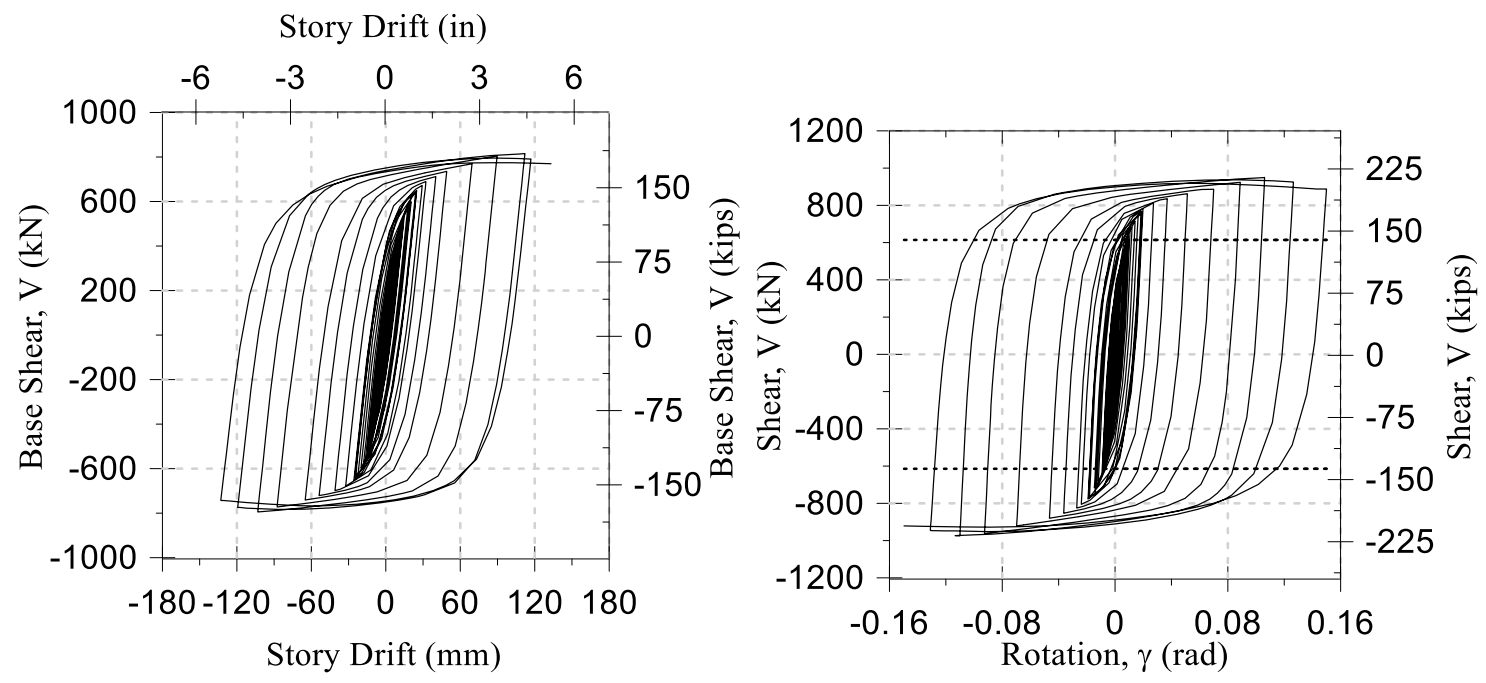

b) Shear link model 17 

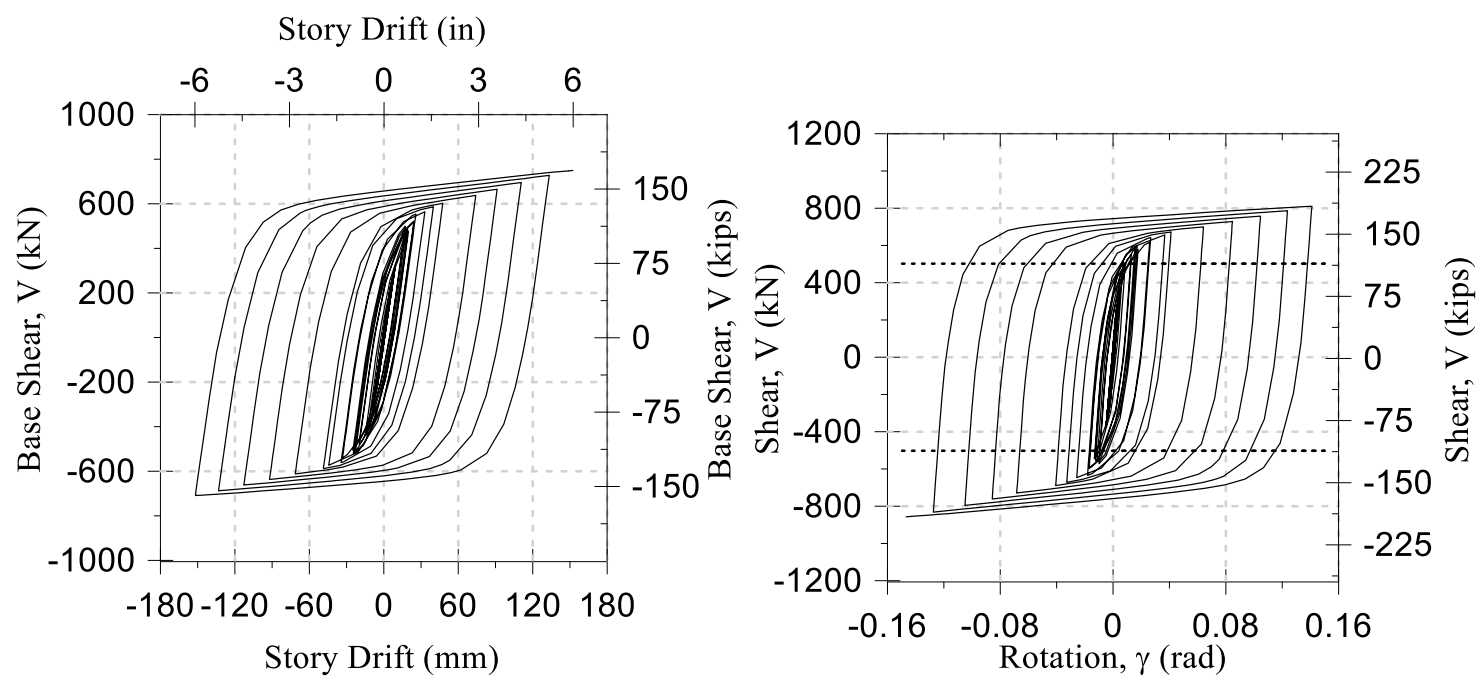

c) Shear link model 18

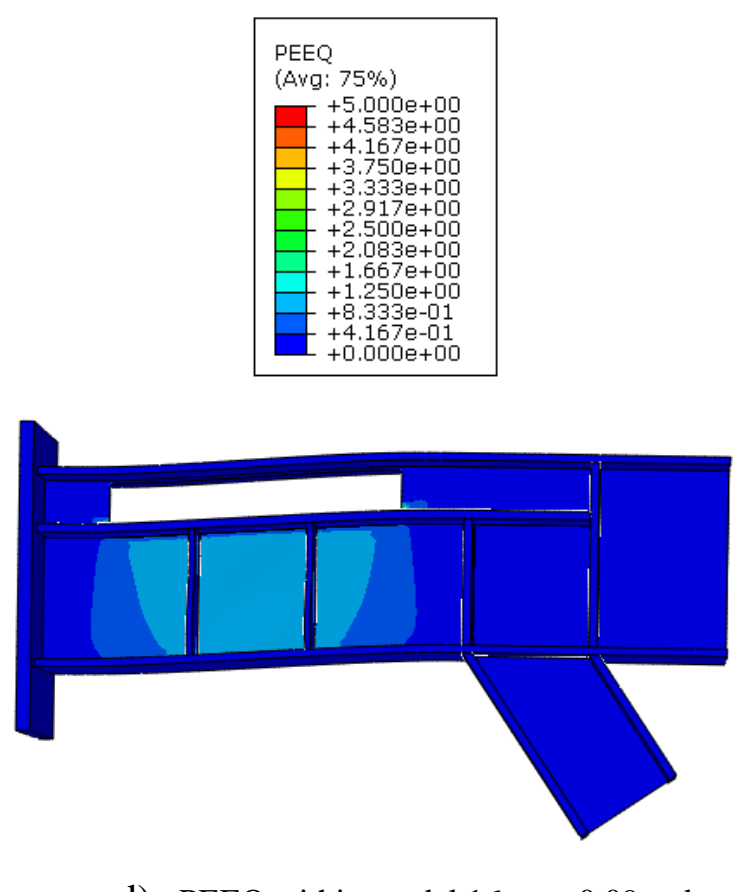

d) PEEQ within model 16 at $\gamma=0.09 \mathrm{rad}$ 


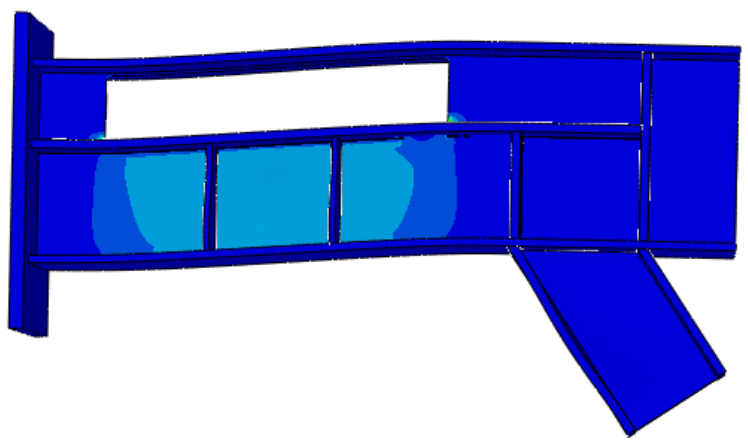

e) PEEQ within model 17 at $\gamma=0.09 \mathrm{rad}$

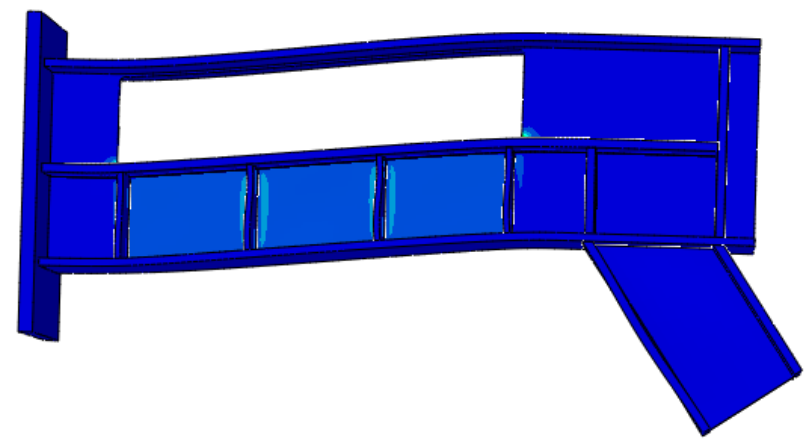

f) PEEQ within model 18 at $\gamma=0.09 \mathrm{rad}$

Figure 5.13 Models 16 to 18 results

\subsubsection{Tuned Common Sections}

The important feature of the link in T-EBF is the ability to tune the strength of a large variety of sections that otherwise cannot be used as links in D-EBF. In this part, links with a range of sections and depths are selected and designed as part of the T-EBF. Table (5.11) contains the dimensions and strength properties for the original sections used in this analysis, while Table (5.12) describes the dimensions and strength properties of the tuned sections. 
Table 5.11 Common sections' properties (in.)

\begin{tabular}{|c|c|c|c|c|c|c|c|}
\hline & $\mathrm{d}$ & $\mathrm{t}_{\mathrm{w}}$ & $\mathrm{t}_{\mathrm{f}}$ & $\mathrm{b}_{\mathrm{f}}$ & $\mathrm{M}_{\mathrm{p}}$ & $\mathrm{V}_{\mathrm{p}}$ & $\begin{array}{c}\text { Stiffeners } \\
\text { spacing } \\
(\mathrm{S})\end{array}$ \\
\hline $\mathrm{W} 14 \times 74$ & 14.2 & 0.45 & 0.785 & 10.1 & 525 & 192 & 12.25 \\
\hline $\mathrm{W} 16 \times 67$ & 16.3 & 0.395 & 0.665 & 10.2 & 542 & 193 & 10.25 \\
\hline $\mathrm{W} 18 \times 76$ & 18.2 & 0.425 & 0.68 & 11.0 & 679 & 232 & 11.0 \\
\hline $\mathrm{W} 24 \times 146$ & 24.7 & 0.65 & 1.09 & 12.9 & 1744 & 482 & 16.75 \\
\hline
\end{tabular}

Table 5.12 Tuned Common sections' properties (in.)

\begin{tabular}{|c|c|c|c|c|c|c|c|c|c|c|c|c|}
\hline & & & & & & & & & & & & \\
\hline
\end{tabular}
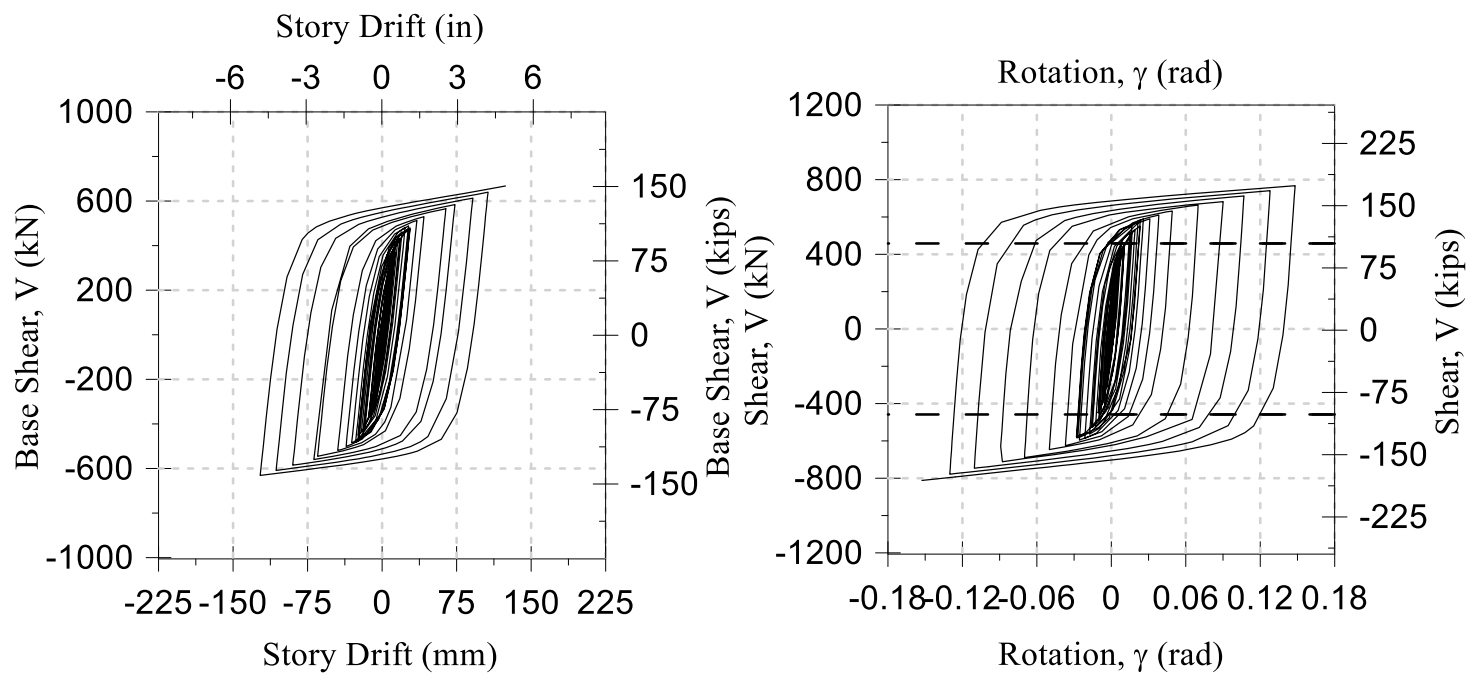

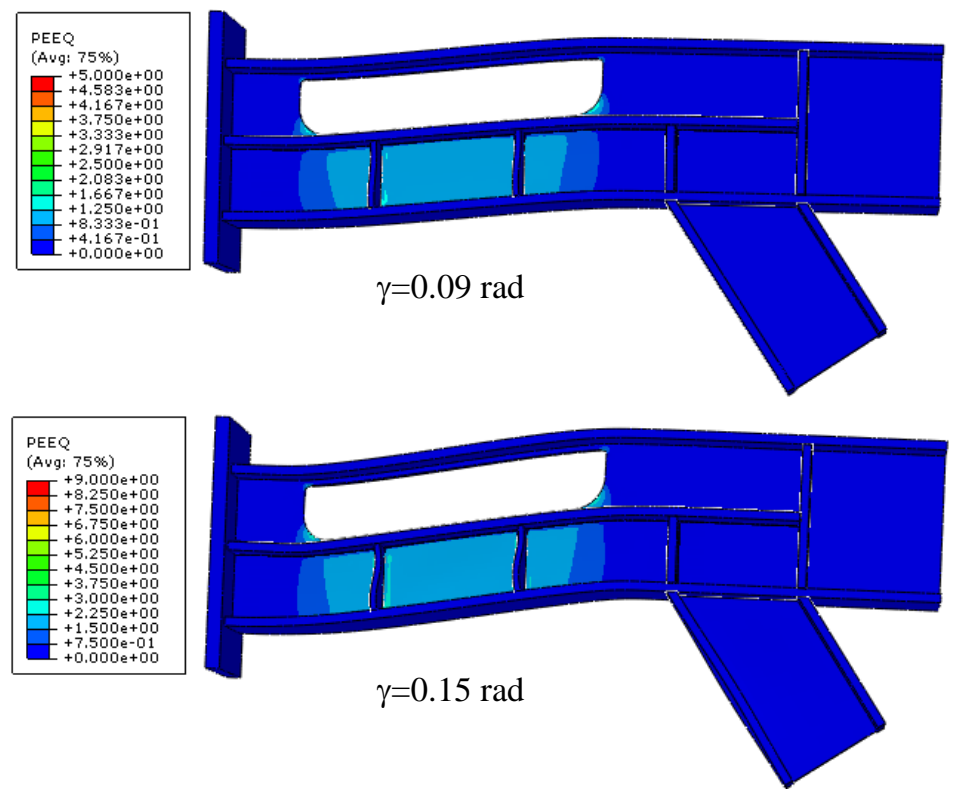

a) W14x74 section

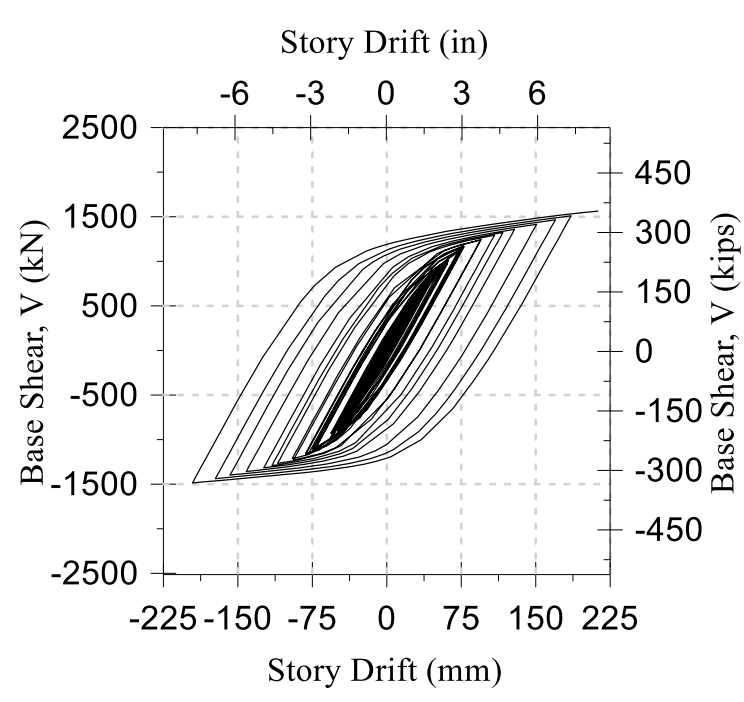

Rotation, $\gamma(\mathrm{rad})$
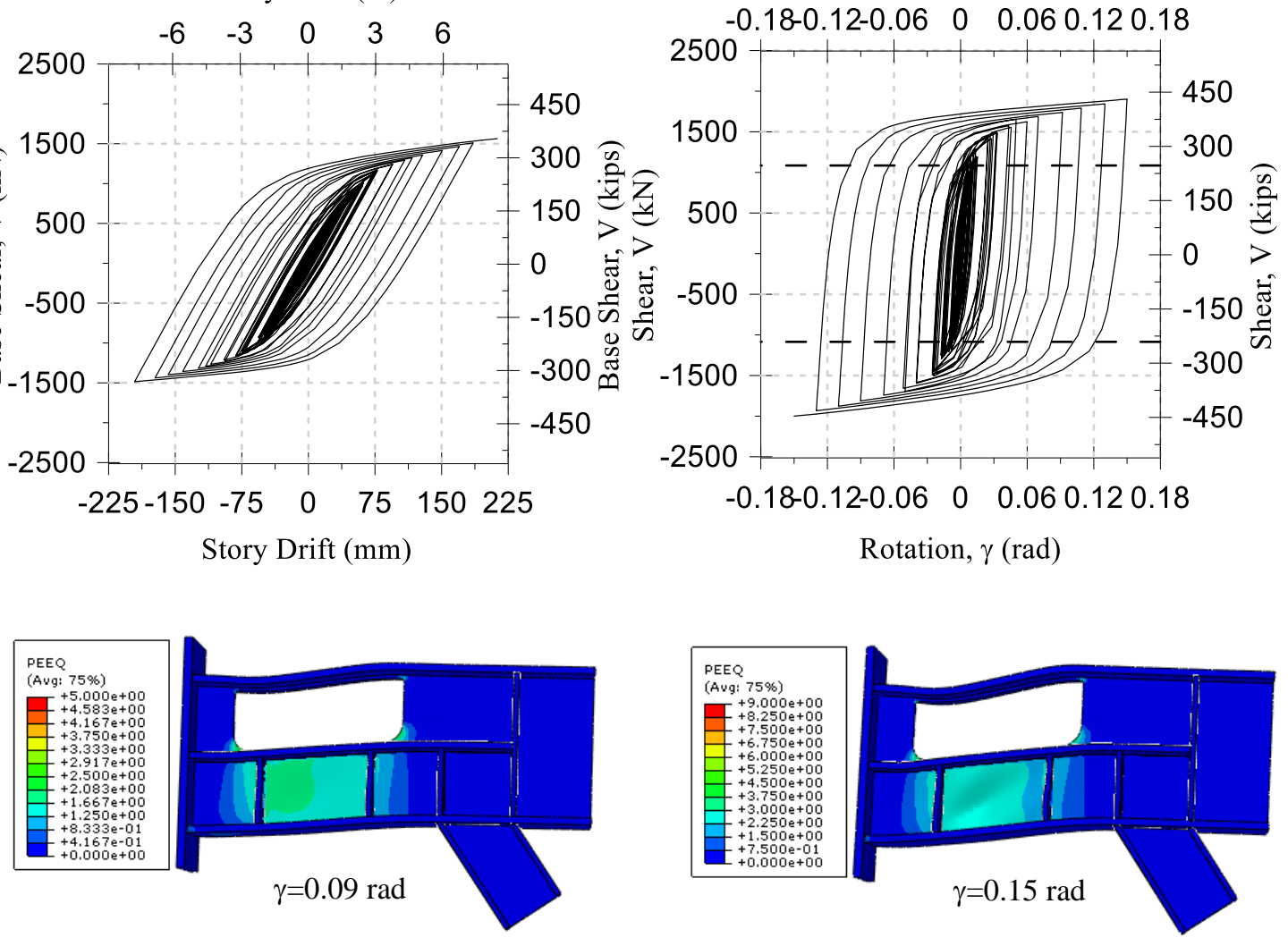

b) $\mathrm{W} 24 \mathrm{x} 146$ 

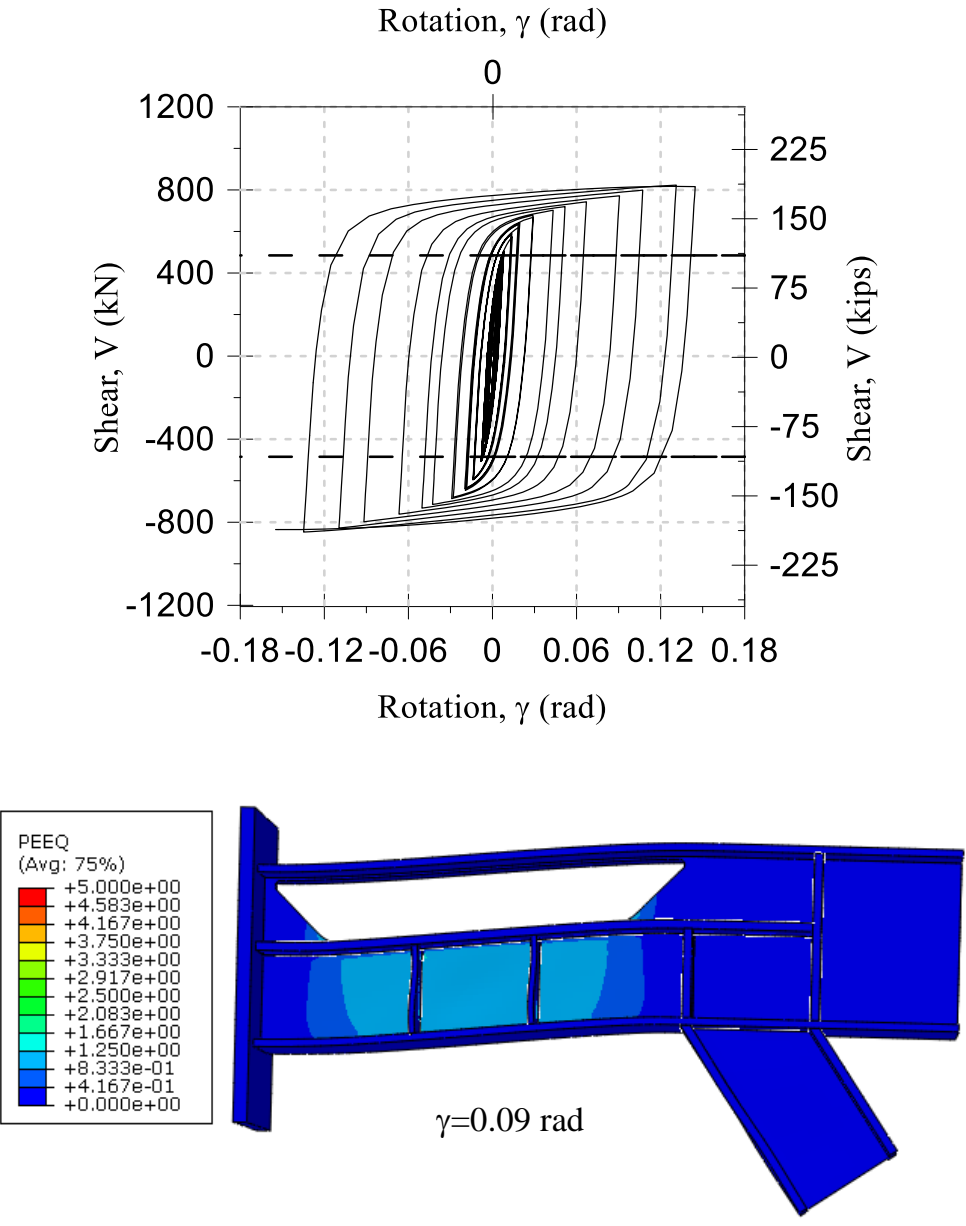

c) $\mathrm{W} 16 \times 67$ 

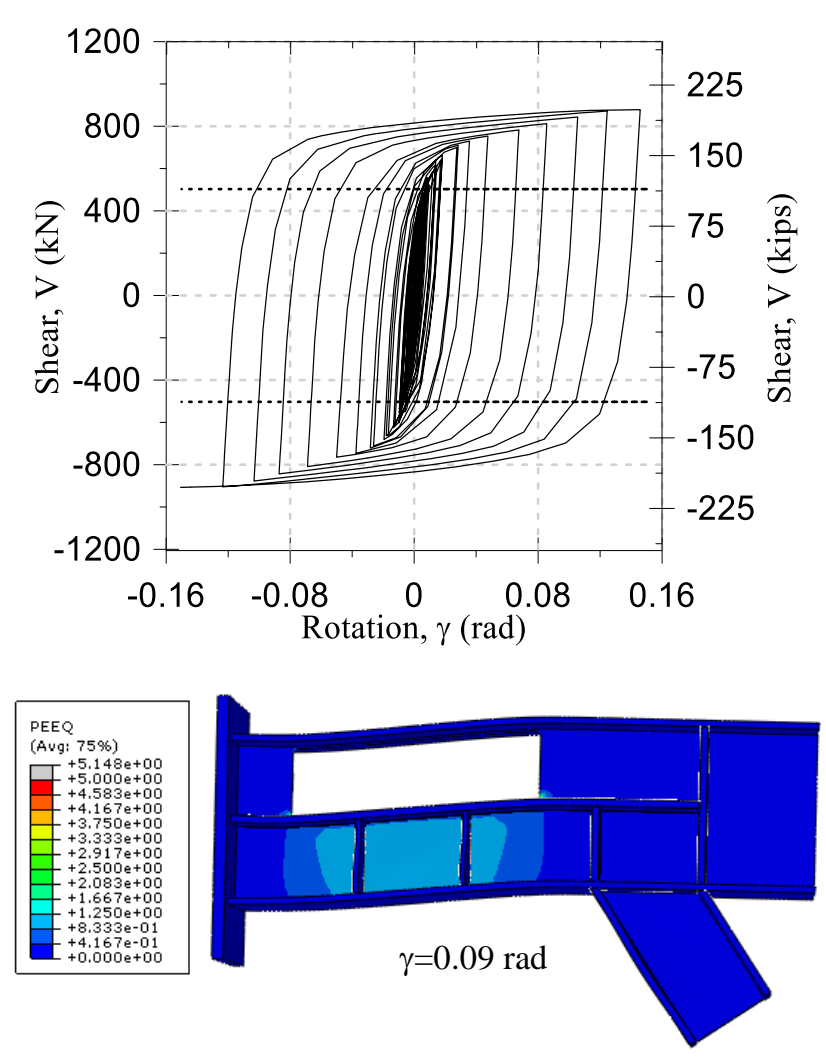

d) $\mathrm{W} 18 \times 76$

Figure 5.14 Models of common sections

Figure (5.14) illustrated that reduction in the web section in T-EBF can achieve the desired rotation angle with the target overstrength factor for different sections and depths. It can also be possible to maintain the story drift through changing the parameters. Sections with deep depth can successfully be used as links in T-EBF. Figure (5.14b) shows section W24 works as a link and can achieve the desired rotation, reaching up to 0.15 rad with slight web buckling that did not show any strength degradation. 
The sections involved in this study meet the requirements regarding compactness ratio given in the AISC seismic design specifications, as shown in Table (5.13)

Table 5.13 Sections compactness ratio

\begin{tabular}{|c|c|c|c|c|c|c|}
\hline \multirow{2}{*}{$\begin{array}{c}\text { Compactness } \\
\text { limitations }\end{array}$} & \multicolumn{3}{|c|}{ Web Compactness } & \multicolumn{3}{c|}{ Flange compactness } \\
\cline { 2 - 7 } & \multicolumn{2}{|c|}{$\frac{h}{t_{w}} \leq 1.57 \sqrt{\frac{E}{R_{y} F_{y}}}=36$} & \multicolumn{3}{c|}{$\frac{b_{f}}{2 t_{f}} \leq 0.4 \sqrt{\frac{E}{R_{y} F_{y}}}=9.2$} \\
\hline
\end{tabular}

\subsection{Beam Outside the link}

As stated in previous chapters, the beam outside the link is designed to remain in elastic stage and the link is the only part to behave inelastically. In D-EBF configuration, the panel of brace-to-link connection can be instable and goes beyond the yield point. Therefore, early research by Popov suggested to provide two vertical stiffeners at this region to avoid local stress concentrations as well as buckling and crippling (Popov, etc 1977).

The results of the numerical analysis demonstrate that a very limited area in the brace connection panel can behave beyond the yield point. The von Mises stress distributions were plotted in Figure (5.15) at links rotation of $0.09 \mathrm{rad}$. The gray contours shown in the figure represent the yielded area in the various models. 

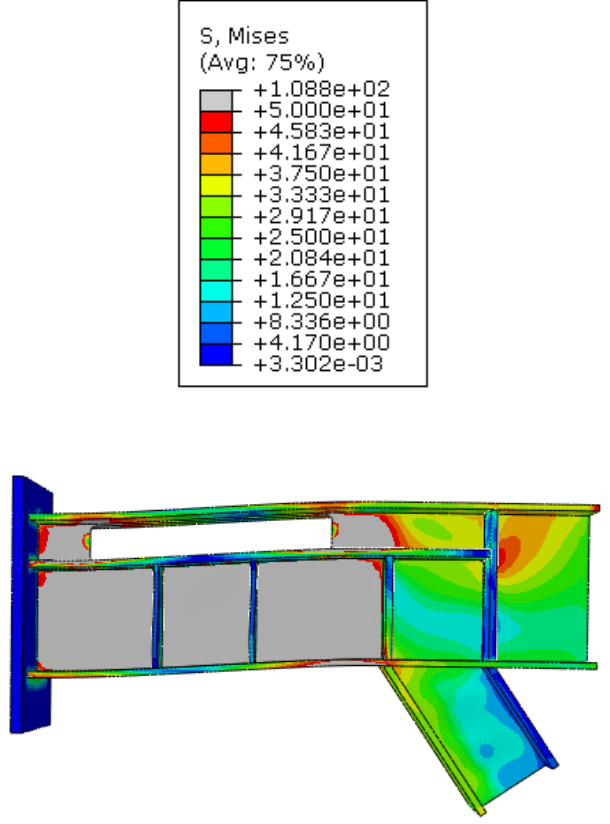

a) Model 16

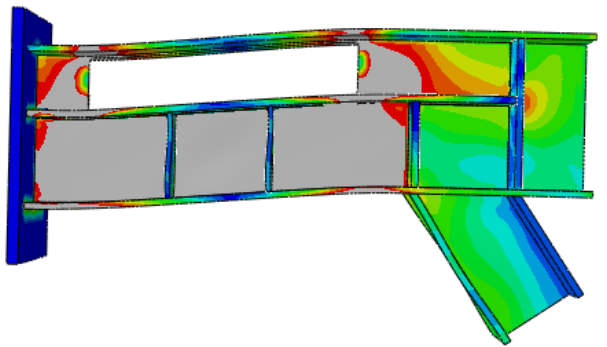

b) Model 17

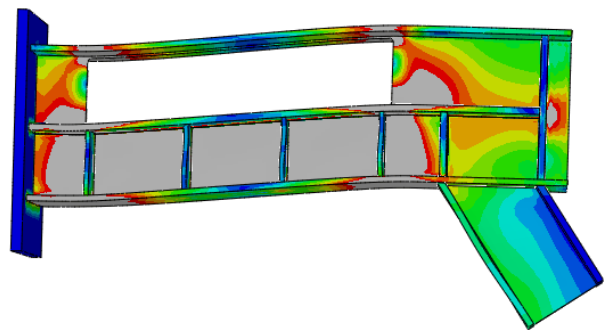

c) Model 18 


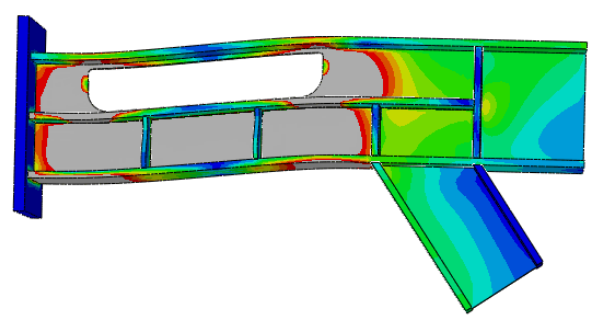

d) $\mathrm{W} 14 \times 74$

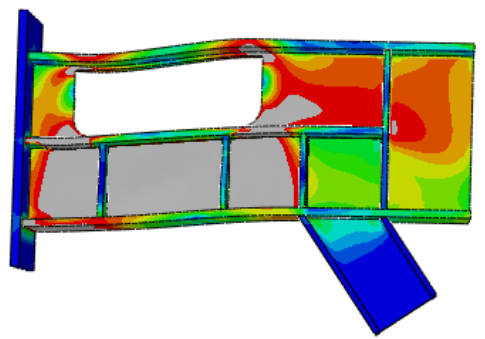

e) $\mathrm{W} 24 \mathrm{x} 146$
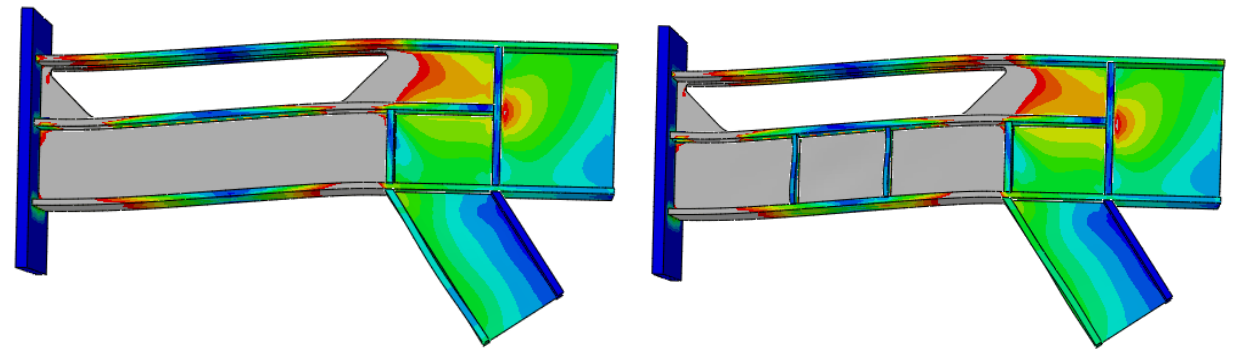

f) $\mathrm{W} 16 \times 67$

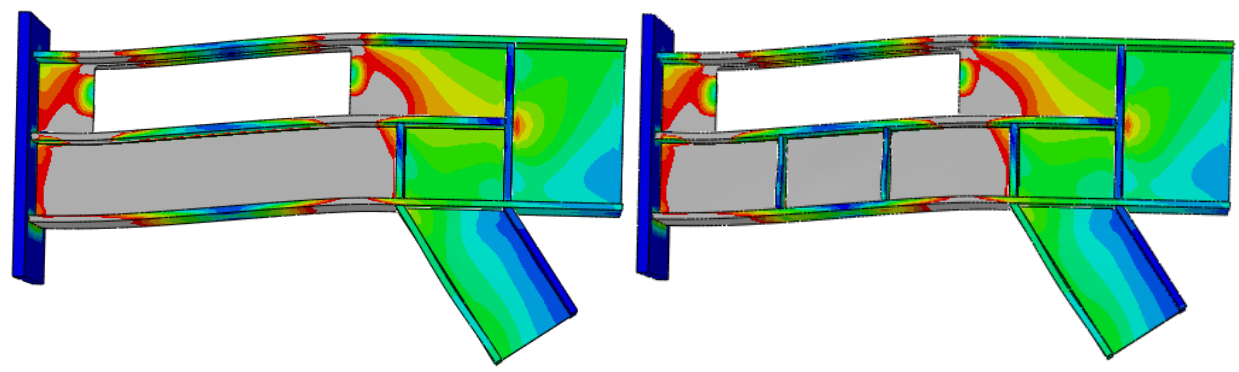

g) $W 18 \times 76$

Figure 5.15 von Mises stress distributions 


\section{Chapter 6: Design Recommendations}

The AISC seismic design provisions were used as the basis for developing the eccentrically braced frame. In the previous chapter, primary link parameters in T-EBF were analyzed and studied for various types of sections. In this chapter, design recommendations for T-EBF will be presented based on the work carried out in this research. The suggestions and recommendations represent the results and observations of this research.

\subsection{Design Loads}

The design load combinations used to calculate the EBF member forces are in accordance with ASCE/ SEI 7 (2010). Structures must be able to resist different load combinations of gravity and lateral seismic forces. The following load combinations related to the Load and Resistance Factor Design (LRFD) are considered;

$1.2 D+1.0 E+0.5 L+0.2 S$

$0.9 D+1.0 E$

Where the symbols are:

L: Live load

D: Dead load

E: Earthquake load

S: Snow load 
The Equivalent Lateral Force (ELF) procedure outlined in ASCE 07-2010 (section 12.8) will be described briefly as it is a simplified procedure for seismic structure design and is useful for studying the performance of drift-controlled buildings. Here, the actual earthquake forces are modified into equivalent static design forces. The first mode from vibration analysis is the mode that governs the design, and the structure mass is active in the first mode. Seismic mass (W) is calculated as the dead and live load combinations presented above. The base shear (V) is calculated using equation (6.3). The base shear is then distributed vertically to the building floors. The structural mass is

lumped at the floors, as expressed in equation (6.4), which also depends on the floor level and story height.

$V=C_{s} W$

$F_{x}=\frac{w_{x} h_{x}^{k}}{\sum_{i=1}^{n} w_{i} h_{i}^{k}} \cdot V$

\section{V: Base Shear}

W: The effective seismic weight

$\mathrm{C}_{\mathrm{s}}$ : Seismic response coefficient (Section 12.8.1.1, ASCE 07-2010)

$F_{x}$ : Lateral seismic force at level $x$

$\mathrm{W}_{\mathrm{i}}$ and $\mathrm{w}_{\mathrm{x}}$ : The portion of the seismic mass assigned to level $\mathrm{i}$ or $\mathrm{x}$.

$h_{i}$ and $h_{x}$ : Height (ft) from the base to level $x$ or $i$.

$\mathrm{k}$ : An exponent related to the structure period. 
The structure fundamental period can be approximately estimated by the expression shown in equation (6.5) (ASCE 07-2010, section 12.8.2.1). Frequency analysis was performed using FEA to determine the EBF, T-EBF fundamental period. Results from FEA shown in Figure (6.1) represent the EBF (2D) and T-EBF (3D) first modes as well as the structure frequencies. These results were compared together, and they are compatible.

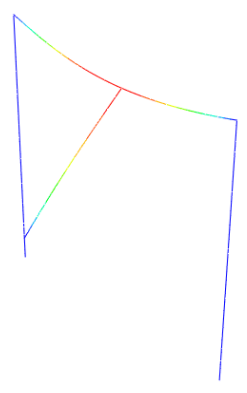

a) $\mathrm{EBF} 1^{\text {st }}$ mode (frequency $(f=6.102)$

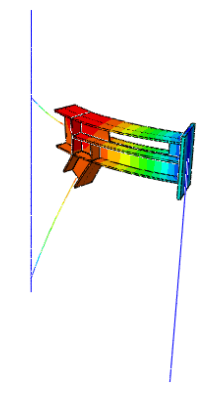

b) T-EBF $1^{\text {st }}$ mode (frequency $(f=6.124)$

Figure 6.1 Fundamental frame frequency

$$
\begin{aligned}
& T=\frac{1}{6.124}=0.1633(\text { fromFEA }) \\
& T_{a}=C_{1} h_{n}^{x}=0.03 \times 9.474^{0.75}=0.162(\text { ASCE }-07)
\end{aligned}
$$

Other dynamic analysis procedures, such as Model Response Spectrum and Seismic Response History, are also permitted in ASCE-07. 


\subsection{Link Requirements}

\subsubsection{Section Compactness}

AISC seismic design provisions require the link of rolled wide-flange sections to meet seismic compactness requirements. The link should satisfy the high ductility requirements with exception of shear links, link length $\leq 1.6 M_{p} / V_{p}$, in which the flanges of the links are permitted to satisfy moderate ductility requirements.

Equations below demonstrate the limitations of width-to-thickness ratio;

$\frac{b_{f}}{2 t_{f}} \leq 0.32 \sqrt{\frac{E}{R_{y} F_{y}}} \quad$ Highly ductile members

$\frac{b_{f}}{2 t_{f}} \leq 0.40 \sqrt{\frac{E}{R_{y} F_{y}}} \quad$ Moderately ductile members

$\frac{h}{t_{w}} \leq 1.57 \sqrt{\frac{E}{R_{y} F_{y}}} \quad$ Highly ductile members

h: Clear distance between flanges less the fillet or corner radius for rolled shapes, denoted as $\mathrm{T}$ in AISC manual.

\subsubsection{Link Rotation}

Achieving the desired link rotation is the primary parameter used to dissipate earthquake energy within the link in EBF. In general, the link rotation demand should not exceed the link rotation capacity. The target rotation angle for shear links is determined by AISC seismic design provision to $0.08 \mathrm{rad}$. The shear link length is defined in chapter 
2 to be less than $1.6 \mathrm{M}_{\mathrm{p}} / \mathrm{V}_{\mathrm{p}}$ where $\mathrm{M}_{\mathrm{p}}$ is the plastic flexural strength and $\mathrm{V}_{\mathrm{p}}$ is the plastic shear strength.

\subsubsection{Link Length}

The link length is a significant limitation that can affect the behavior of EBF. In absence of the effective axial loading in the link or low axial force, at which $\mathrm{P}_{\mathrm{u}} / \mathrm{P}_{\mathrm{y}} \leq 0.15$ (LRFD) where $\mathrm{P}_{\mathrm{u}}$ is the required axial strength and $\mathrm{P}_{\mathrm{y}}$ is the nominal axial yield strength, the link length can be determined according to the architectural requirements. The effective length ( $\left(\mathrm{e}^{\prime}\right)$ is related to the end distance, ED, and the whole link length (e) by the following equation (6.9)

$e^{\prime}=e-2 \times E D$

\subsection{Link Design}

\subsubsection{Member Size}

The link section size can be selected by estimating the required shear forces and then determining the section that has the near nominal shear capacity. The design of the link in conventional EBF, when the link and the beam have the same section, must take into the account the yield and strain hardening of the link developed during the inelastic action. The amplified seismic forces resulting from link hardening can be calculated using the suggested overstrength factor which is defined as the ratio between the maximum shear capacity to the plastic shear strength, as given by equation (6.10). The link shear forces can be evaluated using the ELF described in this chapter through vertical distribution of the base shear on the floors. 


$$
\Omega=\frac{V_{\max }}{V_{p}}
$$

\subsubsection{Link Strength}

Reduction in web area has the effect of controlling the strength properties, as shown in earlier chapters. However, strain hardening can increase the end moment as the link experiences larger rotation. Hardening might also increase the flange strain which can cause rapid web buckling after yielding, especially for the unstiffened web. Web buckling increases the possibility of failure due to severe load-strength degradation and eventually the link's ability to dissipate energy. Therefore, estimating the overstrength factor $\Omega$ must be carefully considered to avoid unstable link buckling behavior. For T$\mathrm{EBF}$ in this research, results show that $\Omega$ for shear strength is $1.5 \mathrm{~V}_{\mathrm{p}}$ which is the value used in EBF. In the same way, the maximum nominal moment used to prevent weld fracture and limiting end moment is estimated to be 1.2 times the plastic moment $\left(\mathrm{M}_{\mathrm{p}}\right)$ for cyclic loading of the shear link (Kasai \& Popov, 1986)

nominal shear strenght $=1.5 V_{P}$

$M_{\text {designed }}$ is the larger of $1.2 M_{p}$, or $M=\frac{V_{u} \times e}{2}$

The required shear strength must be less than the design shear strength $\left(V_{u} \leq \emptyset V_{n}\right)$ where $\mathrm{V}_{\mathrm{u}}=$ shear force in link under specified forces; and $\varnothing V_{n}=$ link design shear strength. 


\subsection{Link Configuration}

The recommended configuration of the link in eccentrically-tuned braced frames is based on the results obtained from this research which was experimentally verified in four full-scale specimens, as described in previous chapters. The suggested tuned link configuration is the first experimentally assessed configuration and the first cyclic evaluated. The following sections represent the T-EBF parameters required for consideration in the design.

\subsubsection{End Distance (ED)}

The end distance (ED), which provides area on both sides of the effective panel, can help to control the story drift. It is also a parameter that can determine the length of the link, an important factor in shear link behavior. Provided ED can also keep inelastic strain away from the ends of the link without causing any fracture. Therefore, the ED can ensure that the end plates, welds, columns, and connections behave elastically.

Even though links without ED behave similar to those possessing ED, the minimum ED should be large enough to provide welds in the web-endplate. Based on practical concerns, clear distance of around half an inch should be provided in addition to the weld size in case of fillet welding.

The maximum ED should be less that the section depth.

web weld size $+0.5 \leq E D \leq$ Section depth $(d)$

Stable links with changing ED distance and lacking any signs of buckling offers proof of the ability to flexibly design and choose suitable end distance to accommodate 
the link length.

\subsubsection{Effective Link Length}

The effective link length can be chosen depending on the architectural requirements. However, flexural links are not recommended for use in D-EBF (Popov et ac, 1992). As previously discussed, end distance can be an important parameter to increase or decrease the effective length. Link length can lead to increase in the nominal moment as expressed in equation (6.14).

$$
M=\frac{V_{u} \times e}{2}
$$

Expression (6.14) is important to accommodate the member size. In other words, instead of modifying the link size, increasing the link length can offer a more efficient way to meet link strength requirements (Council, B. S. S., 2006).

\subsubsection{Effective Depth $\mathrm{h}_{\mathrm{w}}$}

One of the most important features of T-EBF is the ability to change the geometry of the link and the effective depth without changing the beam outside the link. The depth of the tuned section works to control the link strength. The plastic shear strength of the tuned section can be calculated using equation (6.16). The section size is selected as the weakest member. The link section size and the opening depth are chosen based on the required shear force in the link. The AISC seismic design shear strength of the link, $\phi_{v} \mathrm{~V}_{\mathrm{n}}$, LRFD, is used in calculate the shear design of T-EBF. 
$\mathrm{V}_{\mathrm{n}}=\mathrm{V}_{\mathrm{p}} . \quad \phi_{\mathrm{v}}=0.9(\mathrm{LRFD})$

$V_{p}=0.6 F_{y} A_{l w}, A_{l w}=\left(d-h_{o}-2 t_{f}\right) t_{w}$

Equation (6.16) includes the parameter $\mathrm{h}_{\mathrm{o}}$, the hole depth, which is important to select the appropriate section size.

\subsubsection{Fillet Radius}

Although the cracks of fillet radius at the opening corner of T-EBF have no effects on the link strength, it should be designed to the desired link rotation without any sign of fracture or damage. The results show that making the opening edge at $45^{\circ}$ and with a suitable fillet radius can prevent cracks in this region. Based on a previous study by Volynkin (2016) and confirmed with this research, the following equation (6.17) is recommended to select an appropriate fillet radius (R) in inch units;

$R=0.5+3.5\left(t_{w}\right)$

\subsubsection{Horizontal Stiffeners}

The horizontal stiffener thickness used in the T-EBF link must be accurately selected according to the developing forces in the link. The horizontal stiffener thickness should be able to resist the axial and shear forces developing in the link due to the bending moment and link shear. The stiffeners must be provided on both sides of the link. The limitations below represent the minimum stiffener thickness that can selected.

the horizontal stiffeners thickness $t_{h s} \geq t_{f}$ 
Flange compactness equations, $0.4 \sqrt{\frac{E}{R_{y} F_{y}}}$, must be checked in selection of the stiffener thickness. The horizontal stiffener welds can be fillet welds on both sides of the web or partial / complete penetration joints.

\subsubsection{End Vertical Stiffeners}

The vertical stiffeners at the diagonal brace end of the link should be designed according to the AISC seismic provision. Full-depth web stiffeners on both sides should be used in the further end, as shown in the T-EBF configuration. The shorter stiffeners should also be attached on both sides with length appropriate to the horizontal stiffeners' level.

the verical stiffeners thickness $\geq 0.75 t_{w}$ or $3 / 8$ (the larger value)

\subsubsection{Intermediate Stiffeners}

Web buckling is an expected failure accompanying the shear links where no vertical stiffeners are provided. The T-EBF configuration has a horizontal stiffener but can also be provided by vertical stiffeners. Web slender and effective depth-to-web thickness play a significant role in occurring the web buckling at low-link rotation. The effective depth can also serve as a detectable parameter for early buckling. Obviously, in this research, some links without intermediate web stiffeners have undergone web buckling prior to reaching target rotation. Therefore, following the AISC provision in 
providing intermediate stiffeners at certain spacing lengths might be applicable for TEBF.

For shear links $(\gamma=0.08 \mathrm{rad}) S \leq 30 t_{w}-\frac{d}{5}$ and Intermediate web stiffeners must be placed at full depth on both sides when the link depth is equal to or larger than $25^{\prime \prime}$ and only on one side of the link web when the depth is less than $25^{\prime \prime}$.

The stiffener size is also limited by the provision, as equation (6.19).

Stiffeners width $\geq\left(\frac{b_{f}}{2}\right)-t_{w}$

\subsection{Design requirements}

\subsubsection{Drift}

The importance of controlling lateral deflection of the structures is attributed to how story drift can affect the components in both the lateral resisting system and nonlateral resisting system of the structure. The frame story drift should be checked to reduce the potential damages of the building during an earthquake. The drift is calculated in the ASCE-07 2010 in accordance with equation (6.20). In this expression, amplified displacement, $\delta_{\mathrm{x}}$ can be determined by a structural elastic analysis, $\delta_{x e}$, and $\mathrm{I}_{\mathrm{e}}$ is the importance factor (section 11.5.1).

$\delta_{x}=\frac{C_{d} \delta_{x e}}{I_{e}}$

$C_{d}$ :the deflection amplification factor 
The allowable story drift $\Delta$ is limited to the value from table 12.12-1 (ASCE-07) based on the risk category and story height $h_{x x}$.

$\Delta=0.02 \mathrm{~h}_{\mathrm{sx}}$

\subsubsection{Welds Demand}

The welds in T-EBF must follow specification of the Structural Welding CodeSeismic Supplement, AWS. The link-to-column connection in EBF is expected to experience high shear and moments at the both link ends. Weld designs need to be drawn according to calculation of the forces developed due to link hardening. Fillet welds can be used in the design details for the links, brace, and stiffeners. The demand critical welds are determined in the following locations within the EBF, as required by AISC 341;

1. Groove welds at column splices.

2. Welds at column-to-base plate connections.

3. Welds at beam-to-column connections.

4. Where links connect to columns, welds attach the link flanges and the link web to the column.

5. In built-up-beams, welds within the link connect the webs to the flanges.

\subsubsection{End Plate, EP}

The design procedure of the end plates presented in the AISC design guide for extended end plates (Murray, T. M., \& Sumner, E. A. (2003)) is used in T-EBF design. The connection must be fully restrained moment connection and able to sustain the 
desired link rotation. Choosing the correct thickness of EP and correct type, size, and number of bolts that can resist the axial and shear forces should be based on reverse loads since the connection is asymmetric.

\subsubsection{Brace-link Connection Design Requirement}

The EBF brace is designed to carry out the reversal axial forces various from tension to compression. In the case of compression, brace buckling should be avoided, while in tension loads, the brace member is required to resist the forces and prevent section fracture and block shear rupture at the brace-link connection. The brace connections must be able to resist forces due to link yielding and strain hardening. AISC seismic provisions (ANSI/AISC 341-16, 2016) require the brace connection be designed with the same forces as the brace. The strain hardening value was suggested to be 1.25 for the bracing connection design in AISC.

\subsubsection{Beam Outside the Link Design}

The beam segment outside the link should be designed based on the amplified loads from the link. In EBF, the beam outside the link might not be adequate to carry the required loads developed from link ultimate forces. The AISC seismic provision is suggested for use as the overstrength factor is equal to 1.1 based on EBF performance and designer reports. The 1.1 factor is used in the design of T-EBF as well.

\subsubsection{Columns}

The design requirements of the column in T-EBF is the same as the requirements used in design of the beam outside the link since they should stay in the elastic stage and 
the link is the only component that deforms inelastically. The 1.1 overstrength factor is also suggested by the AISC seismic provision. 


\section{Chapter 7: Conclusions and Recommendations}

In this study, a promising new EBF configuration has been introduced to improve the seismic performance of the conventional continuous link. The idea is based on the tuned link properties to control the strength of the link so that any section can be tuned to use as a link in EBF. The following conclusion can be presented based on the results obtained from the experimental and numerical analysis of the shear links.

- The experimental and analytical results obtained in this study show that the TEBF with reduced link web significantly improves behavior of the link-to-column connection. This is a promising solution as the controller beam and the link are in the same section and depth, which is preferable in composite beam and slab construction.

- Modification of the opening shapes can reduce or eliminate transition zone cracks. Increasing the transition radius and cutting out the opening at a 45-degree angle eliminates the transition cracks and reduces the plastic local buckling flange, leading to improved link performance.

- The link is asymmetrical; therefore, the rotation capacity, as well as the shear strain of the panels are different. Slight flange local buckling appeared in various locations along the flanges, affecting the strain of the flange and the measured rotations. 
- A novel brace-to-link connection has been also presented for use in T-EBF. The experimental results show that the performance of the bolted brace-to-link connection is similar to that of the welded brace-to-link connection.

- The maximum shear strain plots of the links revealed that small differences can be observed between the two brace connection types (welded and bolted). The rotational stiffness of the bolted brace-to-link connection might be the main reason for the differences since a thick plate is used in the bolted-brace configuration.

- The numerical study of this research shows that the plastic strain distribution is within the effective panel of the link and PEEQ's were very small near the connections compared to the middle web, which is preferable for dissipating energy inside the effective links.

- The link models analyzed in the parametric study illustrate that a wide range of sections can be used in T-EBF and successfully reach target link rotation.

- The thick plate used in the bolted brace has many benefits for EBFs, such as reducing the stress concentration at the area of the brace flange and the link vertical stiffeners, thus potentially solving the misalignment problem observed from in the finite element analysis performed in this study.

- The middle flange, horizontal flange must satisfy the compactness requirements for moderately ductile members 


\section{Recommendations}

T-EBF has demonstrated the benefit of the suggested link configuration to improve link performance and reduce the plastic strain at the link ends and near connections. However, more research is highly recommended to develop the tuned concept and complete the design recommendations for use in design codes. The points below represent some of the recommendations for future work.

- Apply the concept of T-EBF in different EBF configurations, such as K-brace EBF of continuous link-beam section.

Using the web opening configuration in different link types, such as flexural and intermediate links.

- Though many studies use the reduced web section in the moment resisting frame (MRF), the tuned configuration may also apply to such frames by changing the opening geometry to control the strength.

- Studying the economics of using T-EBF in taller buildings compared with replaceable links. 


\section{References}

ANSI, AISC 341-16. (2016). Seismic provision for structural steel buildings. Chicago: American Institute of Steel Construction.

ASCE, 2010. Minimum Design Loads for Buildings and other Structures. In ASCE/SEI 7-10. American Society of Civil Engineers, Reston-VA, USA.

Ashikov, A., Clifton, G. C., \& Belev, B. (2016). Finite element analysis of eccentrically braced frames with a new type of bolted replaceable active link. In New Zealand Society for Earthquake Engineering 2016 Conference.

Berman, J. W., Okazaki, T., \& Hauksdottir, H. O. (2009). Reduced link sections for improving the ductility of eccentrically braced frame link-to-column connections. Journal of structural engineering, 136(5), 543-553.

Chao, S. H., Khandelwal, K., \& El-Tawil, S. (2006). Ductile web fracture initiation in steel shear links. Journal of structural engineering, 132(8), 1192-1200.

Clifton, G. C., M. Bruneau, G. A. MacRae, R. Leon and A. Fussell (2011). "Steel structures from the Christchurch Earthquake series of 2010 and 2011." Bulletin of the New Zealand Society for Earthquake Engineering 44(4): 297-318.

Council, B. S. S. (2006). NEHRP recommended provisions: design examples FEMA 451-Washington. DC, August.

Darwin, D. (1990). "Steel and composite beams with web openings." Steel Design Guide Series No. 2, AISC, Chicago.

Dusicka, P. \& Itani, A.M. (2002), "Behavior of Built-Up Shear Links Under Large Cyclic Deformations," Proceedings of the 2002 Annual Meeting of the Structural Stability Research Council, Structural Stability Research Council, Gainesville, FL.

Dusicka, P., Itani, A. M., \& Buckle, I. G. (2009). Cyclic behavior of shear links of various grades of plate steel. Journal of structural engineering, 136(4), 370-378.

Dusicka, P., \& Lewis, G. (2010). Investigation of replaceable sacrificial steel links. In Proceedings of the 9th US National and 10th Canadian Conference on Earthquake Engineering (Vol. 1659).

Engelhardt, M. D., \& Popov, E. P. (1992). Experimental performance of long links in eccentrically braced frames. Journal of Structural Engineering, 118(11), 30673088 . 
Foutch, D. A., Goel, S. C., \& Roeder, C. W. (1986). Preliminary report on seismic testing of a full-scale six-story steel building. University of Illinois Engineering Experiment Station. College of Engineering. University of Illinois at UrbanaChampaign.

Gulec, C. K., Gibbons, B., Chen, A., \& Whittaker, A. S. (2011). Damage states and fragility functions for link beams in eccentrically braced frames. Journal of Constructional Steel Research, 67(9), 1299-1309.

Hjelmstad, K. D., \& Popov, E. P. (1983). Cyclic behavior and design of link beams. Journal of Structural Engineering, 109(10), 2387-2403.

Hjelmstad, K. D., \& Popov, E. P. (1983). Seismic behavior of active beam links in eccentrically braced frames. NASA STI/Recon Technical Report N, 84.

Imani, R., \& Bruneau, M. I. C. H. E. L. (2015). Effect of link-beam stiffener and brace flange alignment on inelastic cyclic behavior of eccentrically braced frames. AISC Eng. Journal, 52(2), 109-124.

Kanvinde, A. M., Marshall, K. S., Grilli, D. A., \& Bomba, G. (2014). Forensic analysis of link fractures in eccentrically braced frames during the February 2011 Christchurch earthquake: Testing and simulation. Journal of Structural Engineering, 141(5), 04014146.

Kasai, K., \& Popov, E. P. (1986). General behavior of WF steel shear link beams. Journal of Structural Engineering, 112(2), 362-382.

Kim, S. E., \& Lee, D. H. (2002). Second-order distributed plasticity analysis of space steel frames. Engineering Structures, 24(6), 735-744.

Lemaitre, J., \& Chaboche, J. L. (1994). Mechanics of solid materials. Cambridge university press.

Malley, J. O., \& Popov, E. P. (1983). Design considerations for shear links in eccentrically braced frames. NASA STI/Recon Technical Report N, 84.

Mansour, N., Christopoulos, C., \& Tremblay, R. (2011). Experimental validation of replaceable shear links for eccentrically braced steel frames. Journal of Structural Engineering, 137(10), 1141-1152.

Murray, T. M., \& Sumner, E. A. (2003). Extended End-plate Moment Connections: Seismic and Wind Applications. American Institute of Steel Construction. 
Okazaki, T. (2004). Seismic performanc of link-to-column connections in steel eccentrically braced frames (Doctoral dissertation).

Okazaki, T., Arce, G., Ryu, H. C., \& Engelhardt, M. D. (2005). Experimental study of local buckling, overstrength, and fracture of links in eccentrically braced frames. Journal of Structural Engineering, 131(10), 1526-1535.

Okazaki, T., Engelhardt, M. D., Nakashima, M., \& Suita, K. (2006). Experimental performance of link-to-column connections in eccentrically braced frames. Journal of Structural Engineering, 132(8), 1201-1211.

Okazaki, T., \& Engelhardt, M. D. (2007). Cyclic loading behavior of EBF links constructed of ASTM A992 steel. Journal of constructional steel Research, 63(6), 751-765.

Prinz, G. S., \& Richards, P. W. (2009). Eccentrically braced frame links with reduced web sections. Journal of Constructional Steel Research, 65(10-11), 1971-1978.

Ramadan, T., \& Ghobarah, A. (1995). Behaviour of bolted link-column joints in eccentrically braced frames. Canadian Journal of Civil Engineering, 22(4), 745754.

Roeder, C. W., \& Popov, E. P. (1977). Inelastic behavior of eccentrically braced steel frames under cyclic loadings. NASA STI/Recon Technical Report N, 78.

Seif, M., \& Schafer, B. (2013). Design of locally slender structural steel columns. Journal of Structural Engineering, 140(4), 04013086.

Stephens, M., Dusicka, P., \& Lewis, G. (2011). Buckling behavior of shell elements subjected to inelastic reversed cyclic loading. In Structures Congress 2011 (pp. 3080-3091).

Tan, K. G., \& Christopoulos, C. (2016). Development of Replaceable Cast Steel Links for Eccentrically Braced Frames. Journal of Structural Engineering, 142(10), 04016079.

Tong, L., Zhang, Y., Zhang, L., Liu, H., Zhang, Z., \& Li, R. (2018). Ductility and energy dissipation behavior of G20Mn5QT cast steel shear link beams under cyclic loading. Journal of Constructional Steel Research, 149, 64-77.

Volynkin, D. (2016). Development and Validation of Shear Links for LCF and EBF Seismic Systems via Testing and Numerical Modelling (Doctoral dissertation, ResearchSpace@ Auckland). 
Yang, M. S. (1982). Seismic behavior of an eccentrically X-braced steel structure. University of California, Earthquake Engineering Research Center. 


\section{Appendix A. Design Calculations}

\section{W18x76}

Calculate the shear strength (AISC Seismic Provision,2016)

$V_{p}=0.6 F_{y} A_{l w}, \quad A_{l w}=\left(d-h_{o}-2 t_{f}\right) t_{w}$

$V_{p}=0.6 \times 50 \times 0.425 \times(18.2-2 \times 0.68-7.5)=119.085$

Shear strength (Steel Design Guide Series 2, Steel and Composite Beams with Web Openings, 2003)

$F_{y}=50 k s i$

$w 18 \times 76\left(d=18.2, t_{w}=0.425, b_{f}=11, t_{f}=0.68, Z=163\right)$

$V_{m}=V_{m b}+V_{m t}$

$V_{m b}=V_{p b} \times \alpha_{v b}$

$V_{m t}=V_{p t} \times \alpha_{v t}$

$\alpha_{v}=\frac{\sqrt{6}+\mu}{v+\sqrt{3}} \leq 1.0$

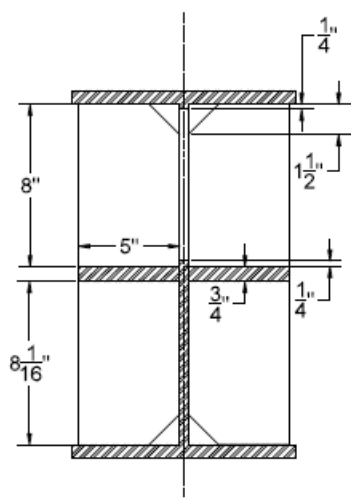

$V_{m}$ : Maximum nominal shear capacity at the location of an opening $V_{m b}, V_{m t}$ : Maximum nominal shear capacity of bottom and top tees respectively $\alpha_{v}$ : Ratio of maximum nominal shear capacity to plastic shear capacity of a tee. $v$ : Aspect ratio of tee

$S, S_{b}, S_{t}$ : Depth of tee, bottom and top respectively.

$a_{o}$ :Length of opening

Top tee

$\mu=\frac{2 p_{r} d_{r}}{V_{p} S}=\frac{2(0) d_{r}}{V_{p} S}=0 \quad\left(p_{r}=A_{r} f_{y}\right), A_{r}=0$

$\alpha_{v t}=\frac{\sqrt{6}+\mu}{v+\sqrt{3}} \leq 1$ 


$$
\begin{aligned}
& v_{t}=\frac{a_{o}}{S_{t}}=\frac{27.8125}{0.9375}=29.667 \\
& \alpha_{v t}=\frac{\sqrt{6}+0}{29.667+\sqrt{3}}=0.078 \\
& V_{p t}=\frac{f_{y} t_{w} S}{\sqrt{3}}=\frac{50(0.425)(0.9375)}{\sqrt{3}}=11.502 \\
& V_{m t}=\alpha_{v} V_{p t}=0.078(11.502)=0.8972
\end{aligned}
$$

Bottom tee

$$
\begin{aligned}
& S_{b}=9.75 \\
& \alpha_{v b}=\frac{\sqrt{6}+\mu}{v+\sqrt{3}} \leq 1.0 \\
& \mu=\frac{2 p_{r} d_{r}}{V_{p} S} \\
& \left(p_{r}=A_{r} f_{y}\right) \leq \frac{f_{y} t_{w} a_{o}}{2 \sqrt{3}}=\frac{50 \times 0.425 \times 27.8125}{2 \sqrt{3}}=170.62 \\
& A_{r}=2[0.75 \times 5.0]=7.5 \\
& S^{\prime}=S-\frac{A_{r}}{2 b_{f}}=9.75-\frac{7.5}{2(11)}=9.41 \\
& P_{r}=f_{y} A_{r}=50(7.5)=375 \\
& \frac{f_{y} t_{w} a_{o}}{2 \sqrt{3}}=170.5 \\
& \mu=\frac{2 p_{r} d_{r}}{V_{p} S}, S=9.75 \\
& V_{p b}=\frac{f_{y} t_{w} S}{\sqrt{3}}=\frac{50(0.425)(9.75)}{\sqrt{3}}=119.62 \\
& \mu=\frac{2 \times 170.62 \times 9.75}{119.62 \times 9.41}=2.955 \\
& v=\frac{a_{o}}{S^{\prime}}=\frac{27.8125}{9.41}=2.9556 \quad \text { so, } u s e \alpha_{v b}=1 \\
& \alpha_{v b}=\frac{\sqrt{6}+2.955}{2.9556+\sqrt{3}}=1.153>1,0.64 \\
& 2
\end{aligned}
$$




$$
\begin{aligned}
& V_{m b}=V_{p b} \times \alpha_{v b}=1.0(119.62)=119.62 \\
& V_{m}=119.62+0.8972=120.52 \\
& V_{p}^{\prime}=\frac{f_{y} t_{w} d}{\sqrt{3}}=\frac{50 \times 0.425 \times 18.2}{\sqrt{3}}=223.3 \\
& V_{m}=120.52 \leq \frac{2}{3}\left(V_{p}^{\prime}\right)=148.86 \rightarrow \text { Okay }
\end{aligned}
$$

\section{Calculate $\mathbf{M}_{\mathrm{p}}$}

Plastic Modulus Z

$$
\begin{aligned}
A_{1}=0.68 & \times 11+0.25 \times 0.425+0.25 \times 0.425+10.425 \times 0.5296=13.213 \\
A_{2}= & 0.68 \times 11+8.09 \times 0.425+10.425 \times 0.2204=13.213 \\
Z=0.68 \times 11 & \times 8.87+0.25 \times 0.425 \times 8.4046+0.25 \times 0.425 \times 0.6546+0.5296 \\
& \times 10.425 \times \frac{0.5296}{2}+0.2204 \times 10.425 \times \frac{0.2204}{2}+8.09 \times 0.425 \\
& \times 4.2654+11 \times 0.68 \times 8.6504=148.4 \mathrm{in}^{3}
\end{aligned}
$$

Sap2000, Section Properties
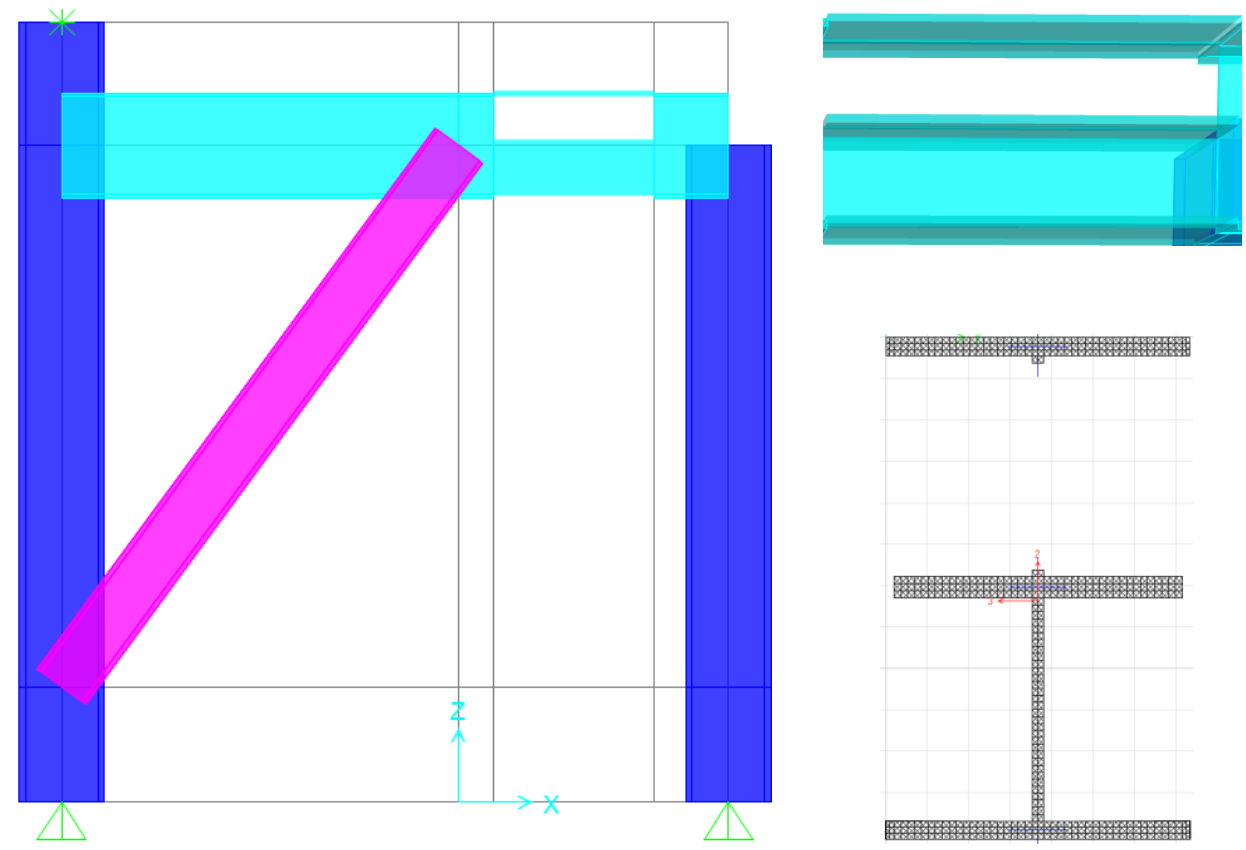


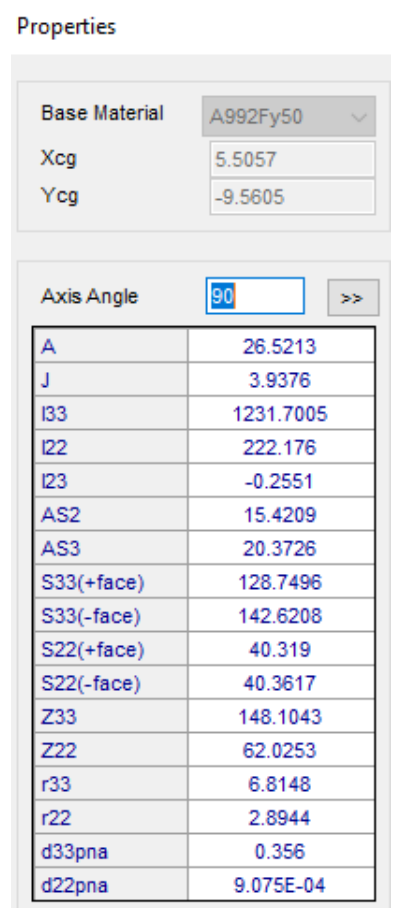

Calculate the moment strength (AISC Seismic Provision,2016)

$M_{p}=F_{y} Z$

$M_{p}=148.4 \times 50=7420$ kips. in $=618.334$ kips. $f t$

Moment strength (Steel Design Guide Series 2, Steel and Composite Beams with Web Openings, 2003)

$$
\begin{aligned}
& h_{o}=7.5 \\
& Z=163 \\
& e_{o}=\frac{18.2}{2}-\left(\frac{7.5}{2}+0.25+0.68\right)=4.42 \\
& M_{p}=F_{y}\left(Z-\frac{h_{o}^{2} t_{w}}{4}-h_{o} e_{o} t_{w}+\frac{\left(t_{h s}\right)^{2}\left(b_{f}-t_{w}\right)}{4}\right) \\
& M_{p}=50\left(163-\frac{7.5^{2} \times 0.425}{4}-7.5 \times 4.42 \times 0.425+\frac{(0.75)^{2} \times(11-0.425)}{4}\right)= \\
& 7221 \text { kips. in }=601.75 \mathrm{kips} . \mathrm{ft}
\end{aligned}
$$


link length $(e)=27.8125^{\prime \prime}<1.6 \frac{M_{p}}{V_{P}}=1.6 \frac{7419}{120.52}=98.493^{\prime \prime}$

Initial $\Omega=2: 00$ was chosen to calculate the forces for the fully strain hardened link,

$$
\begin{aligned}
& V_{u}=2 \times 119=238 \text { kips } \\
& e=27.8125^{\prime \prime} \\
& M_{u}=\frac{V_{u} \times e}{2} \\
& M_{u}=\frac{238 \times 27.8125}{2 \times 12}=275.8 \mathrm{kip}-\mathrm{ft} \\
& \quad \mathrm{V}=238 \mathrm{kips}
\end{aligned}
$$$$
\mathrm{M}=276 \text { kip-ft. }
$$

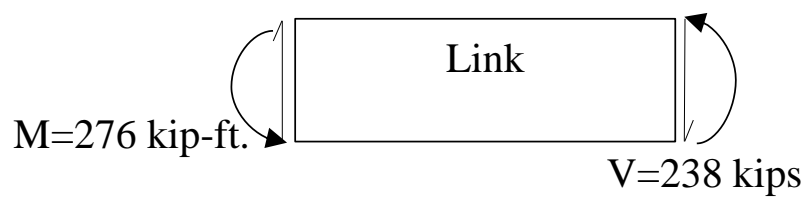

\section{W18x76 Axial loads (Sap2000)}
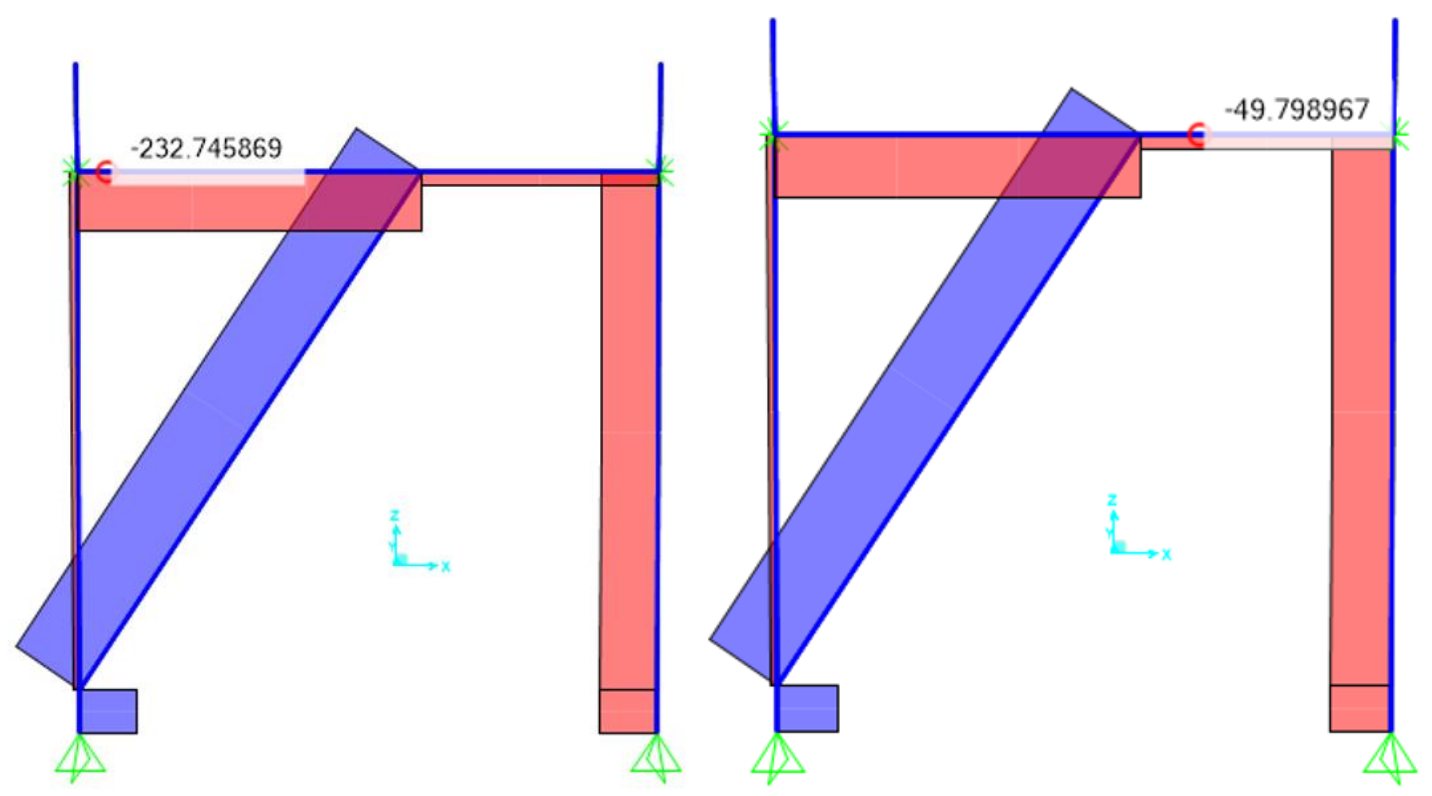


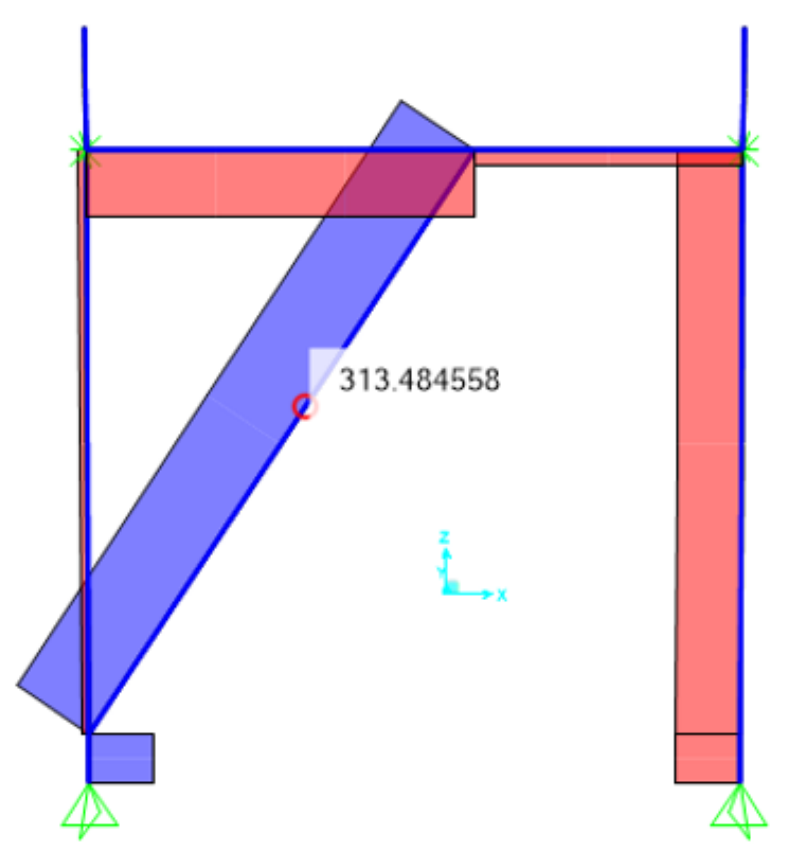

\section{Design Forces}

Link side_\{Sap2000, $N=49.8$ kips, $V=221, M=456.5$ kips $-f t$. $\}$

Flange forces (Axial force + applied moment)

$F_{\text {u,flange }}=\frac{276 \times 12}{18.2-0.68}+49.8=238.85$ kips

Calculate the moment (link side)

$M_{\text {total }}=M+\frac{F \times d}{2}=276+\frac{49.8 \times 18.2}{2 \times 12}=313.8 \mathrm{kip}-\mathrm{ft}$.

Bolts design

Bolts types used and bolts tensile strength $\phi \boldsymbol{r}_{\boldsymbol{n}}$

Bolts Group A

dia $=1 \frac{1}{2}: \phi r_{n}=119$ kips

Bolts Group B

dia $=1 \frac{1}{8}: \phi r_{n}=84.2$ kips

$d i a=1: \phi r_{n}=66.6$ kips 


\section{$\underline{\text { Bolt capacity }}$}

End Plate (Link side)

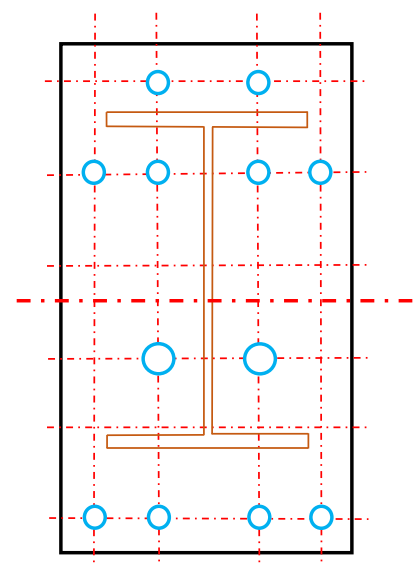

$(2 \times 84.2 \times 19.5) / 12=$

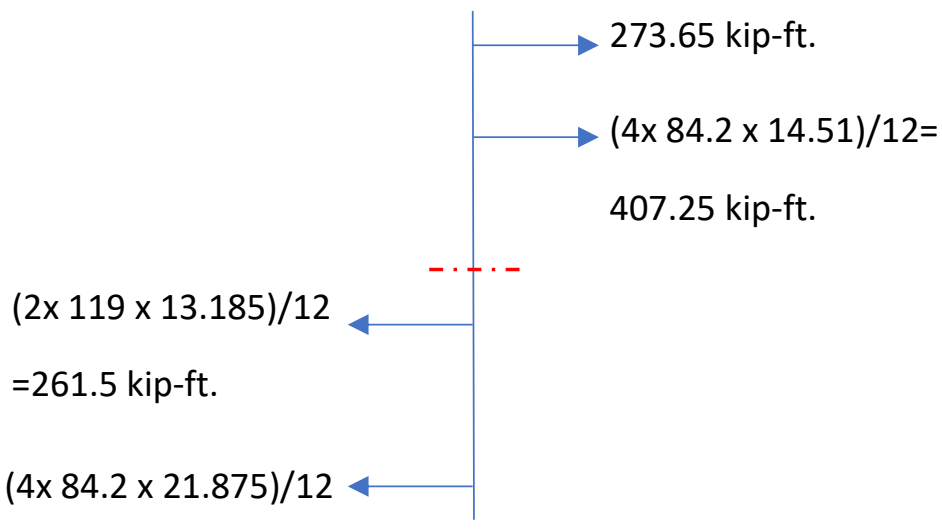

$=614 \mathrm{kip}-\mathrm{ft}$.

$\varnothing M_{n}=\frac{[2 \times 84.2 \times 19.5+4 \times 84.2 \times 14.51]}{12}=681>314 \mathrm{kip}-\mathrm{ft} \quad$ okay

$\varnothing M_{n}=\frac{[4 \times 84.2 \times 21.875+2 \times 119 \times 13.185]}{12}=875.5>314 \mathrm{kip}-\mathrm{ft} \quad$ okay

End Plate Thickness

$$
\begin{aligned}
& S=\frac{1}{2} \sqrt{b_{p} g}=\frac{1}{2} \sqrt{16 \times 5.5}=4.7 \\
& p_{f i, s}=\min [4,4.7] \\
& Y_{p}=\frac{b_{p}}{2}\left[h_{1}\left(\frac{1}{p_{f i}}+\frac{1}{s}\right)+h_{o}\left(\frac{1}{p_{f o}}\right)-\frac{1}{2}\right]+\frac{2}{g}\left[h_{1}\left(p_{f i}+s\right)\right] \\
& Y_{p}=\frac{16}{2}\left[13.185\left(\frac{1}{4}+\frac{1}{4.7}\right)+21.873\left(\frac{1}{4}\right)-\frac{1}{2}\right]+\frac{2}{5.5}[13.185(4+4.7)] \\
& \quad=88.558+41.72=130.27 \\
& t_{p} R e q^{\prime} d=\sqrt{\frac{1.11 \phi M_{n p}}{\phi_{b} F_{y p} Y_{p}}}=\sqrt{\frac{1.1 \times 875 \times 12}{0.9 \times 50 \times 130.27}}=1.4^{\prime \prime}
\end{aligned}
$$

(Note: Check prying action since there were no chance of getting yield in the bolts, so a lessor plate thickness was used $1.25^{\prime \prime}$. 
Prying action

$$
\begin{aligned}
& \text { Required }(t)=\sqrt{\frac{4 \times T \times b^{\prime}}{\emptyset_{b} \times p \times F_{u}(1+\delta \alpha)}} \\
& \text { W18 } \times 76\left[d=18.2, t_{w}=0.425, b_{f}=11, t_{f}=0.68\right] \\
& F_{\text {u,flange }}=238.85 \mathrm{kips} \\
& b^{\prime}=b-\frac{d_{b}}{2} \\
& b=\frac{\left(5.5-t_{w}\right)}{2}=\frac{(5.5-0.425)}{2}=2.5375 \\
& b^{\prime}=2.5375-\frac{1}{2}=2.0375 \\
& a=5.25 \\
& a^{\prime}=a+\frac{d_{b}}{2}=5.25+\frac{1.125}{2}=5.8125 \\
& d^{\prime}=1 \frac{1}{8}+\frac{1}{8}=1 \frac{1}{8}=1.25 \\
& p<2 b=2 \times 2.5375=5.075 \\
& \delta=1-\frac{d^{\prime}}{p}=1-\frac{1.25}{5.075}=0.754
\end{aligned}
$$

The design strength of one bolt is: -

$$
\begin{aligned}
& B=\emptyset \times F_{t} \times A_{b}=0.75(113)(0.994)=84.2 \\
& T=P=238.85 \text { kips }
\end{aligned}
$$$$
\text { Tension } / \text { bolts }=\frac{238.85}{12}=19.9 \quad \text { use } T_{b}=20
$$$$
\text { compute } \alpha=\frac{B}{T}-1=\frac{84.2}{20}-1=3.21
$$$$
\alpha=\frac{3.21\left(\frac{5.8125}{2.0375}\right)}{0.754\left[1-3.21\left(\frac{5.8125}{2.0375}\right)\right]}=\frac{6.0435}{-3.803}=-1.48 \rightarrow|-1.48|=1.48>1
$$

use 1.0 
$\operatorname{Required}(t)=\sqrt{\frac{4 \times T \times b^{\prime}}{\emptyset_{b} \times p \times F_{u}(1+\delta \alpha)}}$

Required $(t)=\sqrt{\frac{4 \times 20 \times 2.0375}{0.9 \times 5.075 \times 65(1+0.754(1.0))}}=0.32 \rightarrow<1.25^{\prime \prime} \quad$ O.K.

\section{$\underline{\text { Weld Design }}$}

Link side

Web welds

$V_{u}=238$ kips

$l_{w}=\frac{d}{2}-t_{f}=\frac{18.2}{2}-0.68=8.42^{\prime \prime}$

$D_{\min }=\frac{238}{2(1.392)(8.42)}=10$

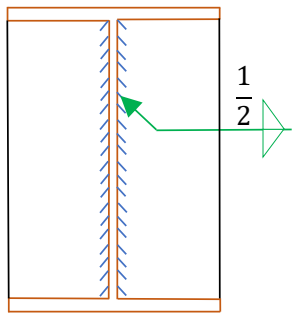

Use $\frac{1}{2} ", \quad 0.5 "$

Flange welds

$F_{u, \text { flange }}=238.85$ kips

According to AISC Design Guide 4 recommends, the minimum design flange force should be 60 percent of the flange yield strength.

$$
\begin{gathered}
\text { Min, } F_{f u}=0.6 \times f_{y} \times b_{f} \times t_{f}=0.6 \times 50 \times 11 \times 0.68=224.4<238.85 \text { kips } \\
\rightarrow \text { use } 238.85 \text { kips } \\
l_{f}=2 b_{f}-t_{w}-2 k=2 \times 11-0.425-2.16=19.415
\end{gathered}
$$

According to AISC, weld strength increases by $50 \%$ due to transverse strength.

$$
\begin{aligned}
& D_{\min }=\frac{238.85}{(1.392)(1.5)(19.415)}=6 " \\
& \text { Use } \frac{8}{16} "=\frac{1}{2} ", 0 .{ }^{\prime \prime}
\end{aligned}
$$

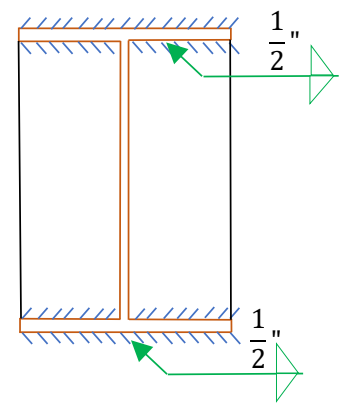




\section{Design of end plates Beam side}

Axial W18

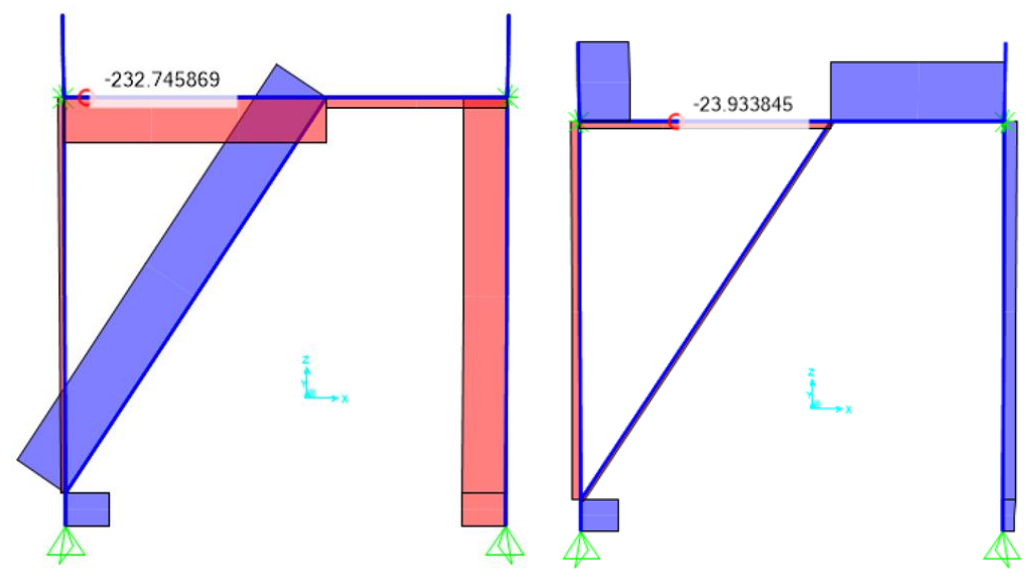

Axial, shear and moment from sap2000

$\mathrm{N}_{\mathrm{u}}=232.75$ kips

$\mathrm{V}_{\mathrm{u}}=24$ kips

$\mathrm{M}=210.68$ kips-ft

Moment W18
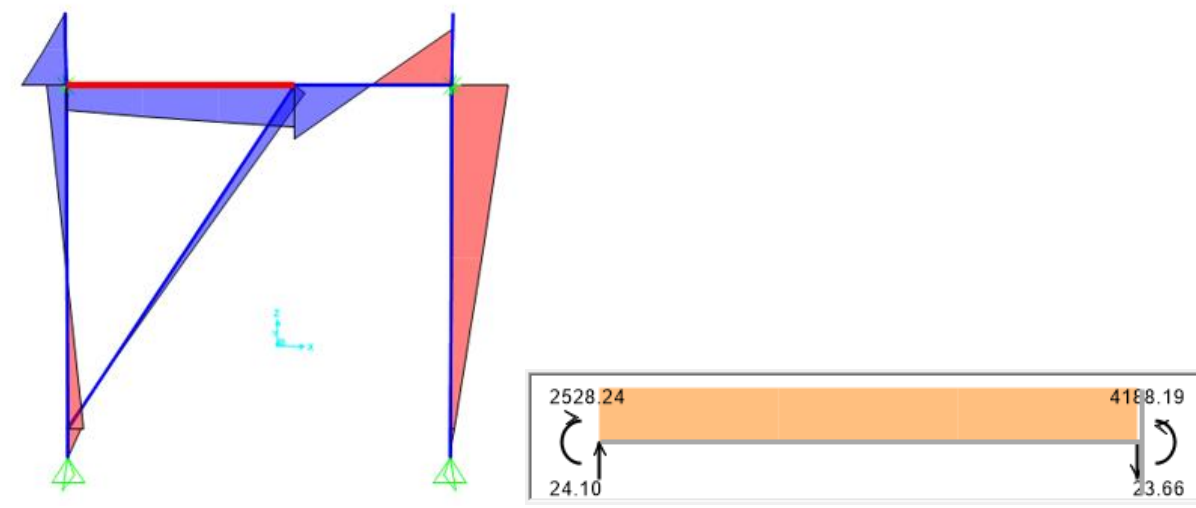

Dist Load (2-dir)

Beam side_\{Sap2000, $N=232.8$ kips, $V=24, M=210.7$ kips $-f t$. $\}$

Flange forces

$F_{u, f l a n g e}=\frac{210.7 \times 12}{18.2-0.68}+\frac{232.8}{2}=260.7$ kips

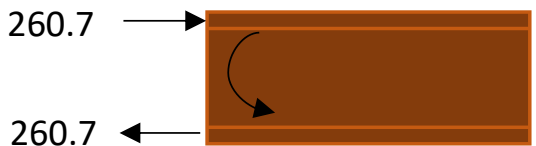

Calculate the moment (Beam side) 


$$
M_{\text {total }}=M+\frac{F \times d}{2}=210.7+\frac{232.8 \times 18.2}{2 \times 12}=387.25 \mathrm{kip}-\mathrm{ft}
$$

End Plate (Beam side)

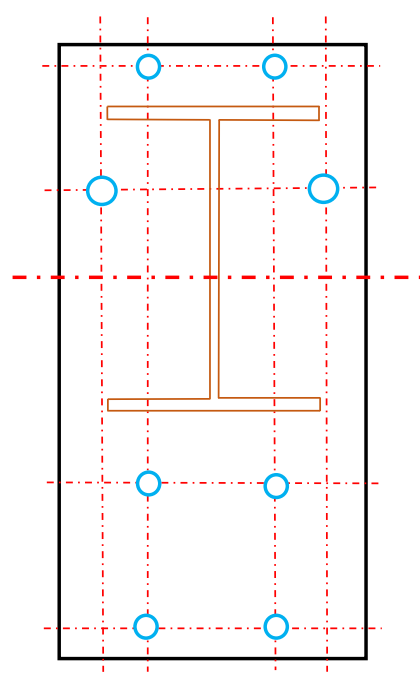

$(2 \times 84.2 \times 19.5) / 12=$ 273.65 kip-ft.

$(2 \times 119 \times 13.121) / 12=$

260.23 kip-ft.
$(2 \times 84.2 \times 19.5) / 12=$

273.65 kip-ft.

$(2 \times 84.2 \times 27.25) / 12=$

382.4 kip-ft.

$$
\begin{gathered}
\emptyset M_{n}=\frac{[2 \times 84.2 \times 19.5+2 \times 119 \times 13.121]}{12}=533.8>350 \mathrm{kip}-\mathrm{ft} \\
\rightarrow \text { okay } \\
\varnothing M_{n}=\frac{[2 \times 84.2 \times 19.5+2 \times 84.2 \times 27.25]}{12}=658>350 \mathrm{kip}-\mathrm{ft} \\
\rightarrow \text { okay } \\
M_{e}=M_{u}+\frac{N_{u} \times d}{2 \times 12}=210+\frac{232.8 \times 18.2}{24}=387.25 \text { kips }-f t
\end{gathered}
$$

Web welds

$V_{u}=24$ kips

$$
\begin{aligned}
& l_{w}=\frac{d}{2}-t f_{b}=\frac{18.2}{2}-0.68=8.42^{\prime \prime} \\
& D_{\text {min }}=\frac{24}{2(1.392)(8.42)}=1.0^{\prime \prime}, \quad \text { use } \frac{1}{2} "
\end{aligned}
$$

Flange Welds

$$
\begin{aligned}
& F_{u, \text { tens }}=260.7 \text { kips } \\
& l_{f}=b_{f}=11 \times 2-0.425=21.575 "
\end{aligned}
$$


$D_{\text {min }}=\frac{260.7}{(1.392)(1.5)(21.575)}=5.8^{\prime \prime}, \quad$ use $\frac{8}{16} "$

Use $\frac{1}{2} "=0.5 "$

$\underline{\text { Shear yielding in plate }}$

$$
\begin{aligned}
& R_{u}=\frac{F_{u, \text { tens }}}{2}=\frac{260.7}{2}=130.35 \\
& \emptyset R_{n}=\emptyset_{1.0} \times 0.6 \times F_{y} \times t_{p} \times b_{p} \\
& R_{n}=1.0 \times 0.6 \times 50 \times 1.25 \times 16=600 \quad>130.35 \quad \text { okay }
\end{aligned}
$$

$\underline{\text { Shear rupture in plate }}$

$$
\begin{aligned}
& \varnothing R_{n}=\emptyset_{0.75} \times 0.6 \times F_{u} \times t_{p} \times\left[b_{p}-2 \times\left(d_{b, 1 \frac{1}{8}}\right]\right. \\
& R_{n}=0.75 \times 0.6 \times 65 \times 1.25 \times[16-2 \times 1.1875]=498.164 \quad>130.35 \quad \text { okay }
\end{aligned}
$$

Design of bolt shear

$$
\begin{aligned}
& V_{u}=\emptyset \mathrm{o} V_{p}=2 \times 119.2=238.4 \text { kips } \\
& \emptyset v_{n}=\emptyset r_{n v} \times n_{b b}=50.7 \times 6=304.2>238.4 \text { okay }
\end{aligned}
$$

\section{Bolt Bearing}

$$
\begin{aligned}
& l_{c}=p f_{o}+p f_{i}+t_{f}=1.625+2.6875+0.68=5 \\
& \emptyset R_{n}=1.2 \times l_{c} \times t_{f} \times f_{u} \times n_{b b} \\
& =1.2 \times 5 \times 0.68 \times 65 \times 6=1591.2>238.4
\end{aligned}
$$

Another bolt bearing eq.

$$
\begin{aligned}
& \emptyset R_{n}=2.4 \times d_{b} \times t_{p} \times F_{u} \times n_{b b} \\
& \emptyset R_{n}=2.4 \times 1.125 \times 1.25 \times 65 \times 6=1316.25 \quad>238.4 \quad \text { o. } k .
\end{aligned}
$$




\section{Brace Collector Side Welds}

Axial load

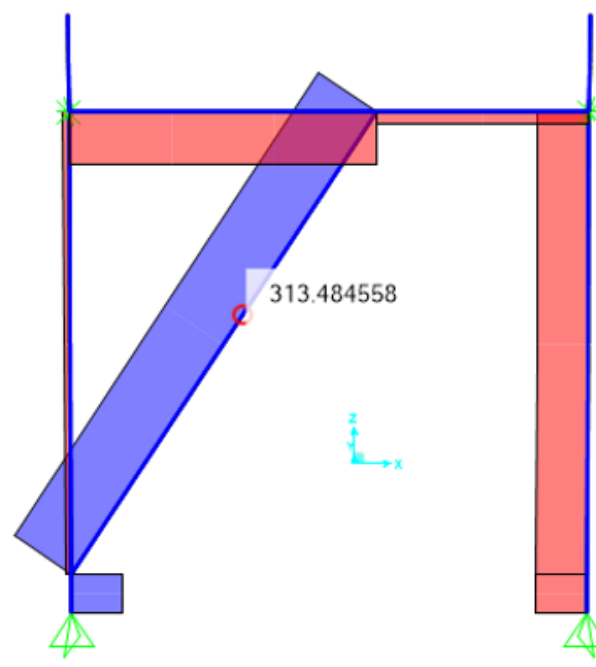

Shear Force

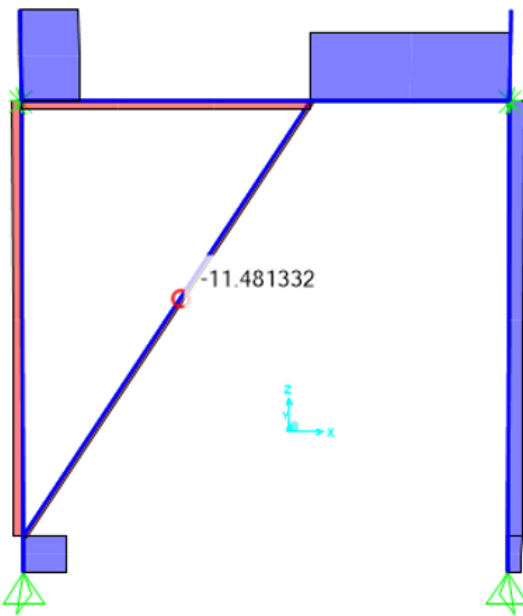

Moments
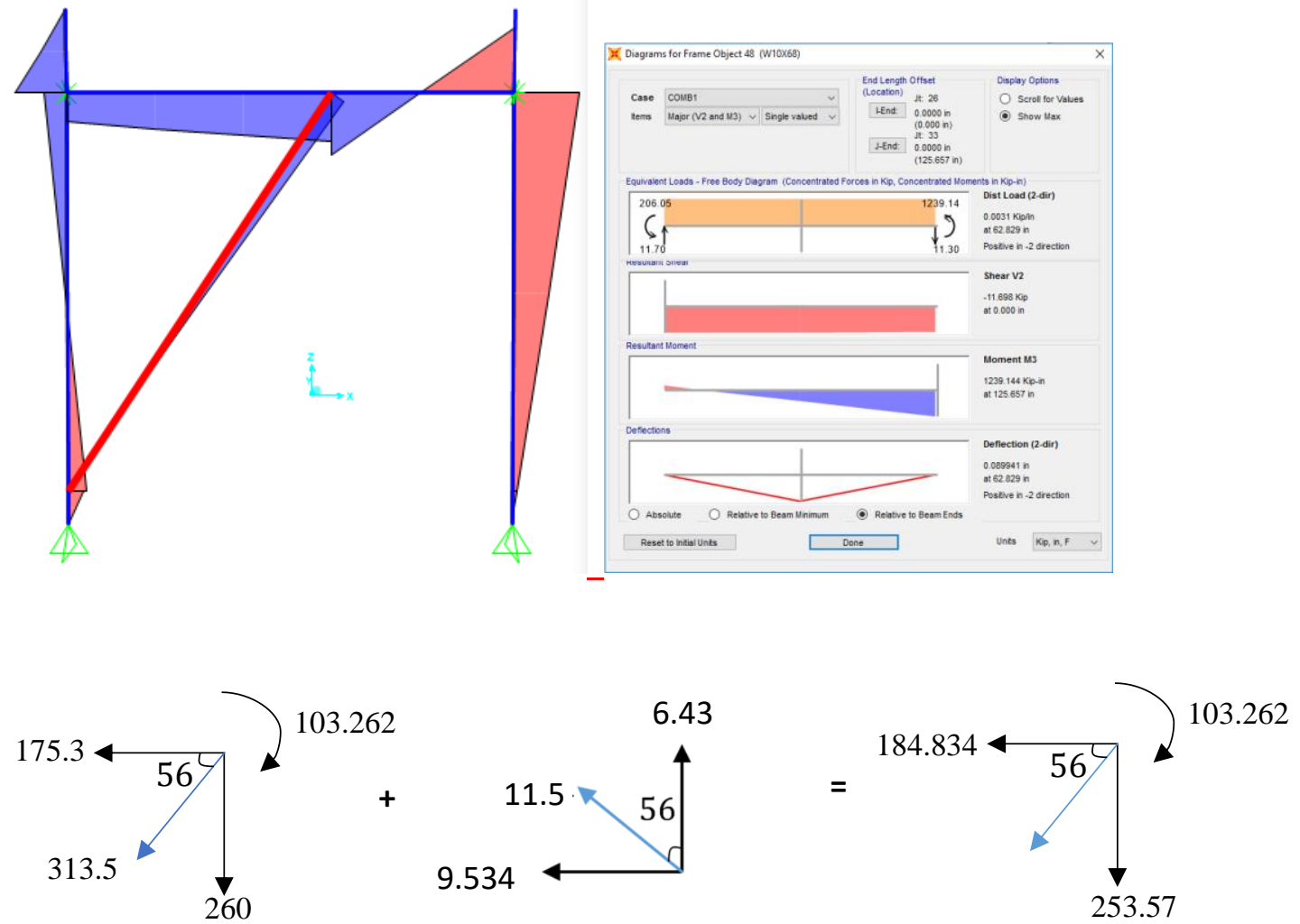
$\underline{\text { Web welds }}$

$$
\begin{aligned}
& V_{u}=184.834 \text { kips } \\
& l_{w}=\frac{d}{2}-t_{f}=\frac{10.4}{2}-0.77=4.43 " \\
& D_{\min }=\frac{184.834}{(1.392)[2 \times(10.1812)-4 \times 1.27]}=\frac{9}{16} ", \quad \text { use } \frac{5^{\prime \prime}}{8}
\end{aligned}
$$

Flange Welds

$$
\begin{aligned}
& \mathrm{Pu}_{\mathrm{u} \text { from axial load }} \quad P_{u) \text { flange }}=253.57 \times\left[\frac{10.1 \times 0.77}{19.9}\right]=99 \\
& \mathrm{Pu}_{\mathrm{u} \text { from } \mathrm{M}} \quad P_{u) \text { flange }}=\left[\frac{103.262 \times 12}{(10.4-0.77)}\right]=128.675 \\
& F=P_{u) \text { axial }}+P_{u) M}=99+128.675=227.675 \\
& D_{\min }=\frac{227.675}{(1.392)\left[2 \times(10.1)-0.47-2 \times \frac{7}{8}\right]}=9.5 ", \quad \frac{10}{16} ", \quad \text { use } \frac{5^{\prime \prime}}{8}
\end{aligned}
$$

Design of stiffeners

$M=276$ kips. $f t$

Axial load $=49.7$

$$
M=F \times 8.76 \rightarrow F=\frac{276 \times 12}{8.725}=379.6 \text { kips. } f t
$$

Total axial load $=49.7+379.6=429.3$

$$
l_{\text {stiff. }}=51.4, \quad l_{\text {total }}=4 \times 51.4=205.6
$$

$\underline{\text { Stiffener-web welds }}$

$$
D_{\min }=\frac{429.3}{(1.392)[205.6]}=1.5^{\prime \prime}, \quad \text { use } \frac{3}{8} "
$$

Stiffener-Endplate welds

$$
D_{\text {min }}=\frac{0.6 \times 50 \times 2 \times 5 \times 0.75}{(1.392)[4 \times 5 \times 1.5]}=5.38 ", \quad \text { use } \frac{1}{2} "
$$

Link end vertical stiffeners-web welds

$$
\begin{aligned}
& F=P_{u) \text { axial }}+P_{u) M}=99+128.675=227.675 \\
& D_{\text {min }}=\frac{227.675}{(1.392)[4 \times 16.8]}=2.5^{\prime \prime}, \quad \text { use } \frac{3^{\prime \prime}}{8}
\end{aligned}
$$

Link end vertical stiffeners-flange welds 
$D_{\text {min }}=\frac{0.6 \times 50 \times 2 \times 5 \times 0.75}{(1.392)[2 \times 2 \times 5 \times 1.5]}=5.4 ", \quad$ use $\frac{1}{2} "$

$D_{\min }=\frac{227.675}{(1.392)[4 \times 8]}=5^{\prime \prime}, \quad$ use $\frac{1^{\prime \prime}}{2}$

$\underline{\text { Beam flange-endplate stiffeners welds }}$

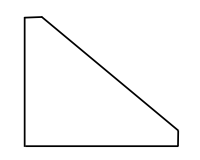

$h_{s t}=10$

$L_{s t}=\frac{h_{s t}}{\tan (30)}=17.3$

$t=t_{w b} \times\left(\frac{f_{y b}}{f_{y s}}\right)=t_{w b} \times 1$

$t=0.425$

Use max. weld size

Plate thickness $=0.425$

Max weld size $=t-\frac{1}{16}=0.425-\frac{1}{16}=0.3625 \quad \rightarrow$ use $\frac{3}{8}$ "weld size

\section{Bolted Brace Connection}
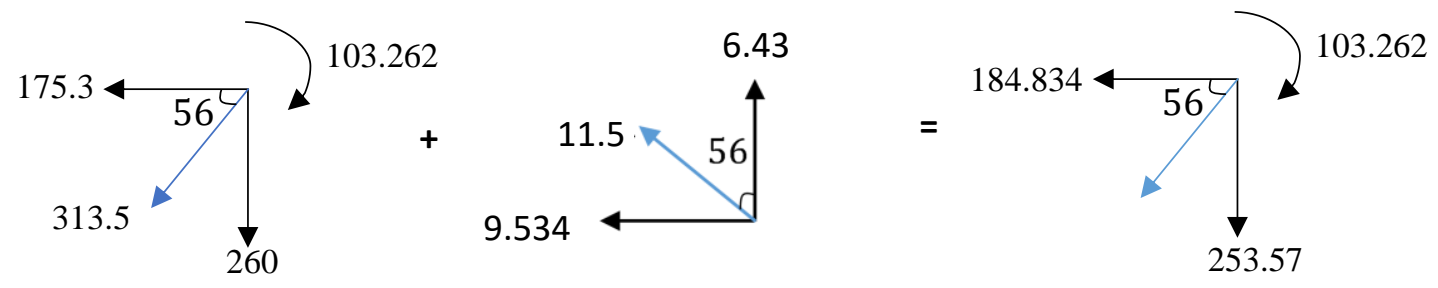

$\underline{\text { Resultant }}$

$F_{x}=184.834$ Kips

$F_{y}=253.57$ Kips adding forces since cyclic load applied

$M=103.262$ kips - ft.

Use bolt type group B-N

Bolt dia.=1" 
Area $=0.785$ inch $^{2}$

Bolt strength

Shear $=40 \mathrm{kips} / \mathrm{bolt}$

Tensile $=66.6 \mathrm{kips} / \mathrm{bolt}$

Check the strength

$\underline{\text { Bolts shear }}$

Bolts shear strenght $=8 \times 40=320$ kips $>184.834 \quad$ Okay

Bearing strength

Plate materials

A572 Grade $50\left(f_{y}=50, f_{u}=65\right)$

Hole dia. $=d+\frac{1}{16}=1+\frac{1}{16}=\frac{17}{16}$

Plate thickness $(t)=1.25^{\prime \prime}$

$l_{c}($ edge $)=l_{e}-\frac{h}{2}=2.5-\frac{\left(\frac{17}{16}\right)}{2}=1.968$

$R_{n}=1.2 l_{c} \times t \times f_{u}=1.2 \times 1.968 \times 1.25 \times 65=192$

Check the upper limit

$R_{n}=2.4 d \times t \times f_{u}=2.4 \times 1 \times 1.25 \times 65=195$

Bolt shear strength controls the design

Plate strength

$$
\begin{aligned}
& P_{n}=f_{y} \times A_{g}=50(10.2 \times 1.25)=382.5 \\
& \emptyset P_{n}=0.9 \times 378.75=344.25 \\
& P_{n}=f_{u} \times A_{e}=65(1.25)\left(10.2-2 \times \frac{9}{8}\right)=645.94 \\
& \varnothing P_{n}=0.9 \times 645.94=581.34
\end{aligned}
$$

$\underline{\text { Block shear }}$

$$
\begin{aligned}
& R_{n}=0.6 F_{u} A_{n v}+U_{b s} F_{u} A_{n t} \\
& A_{g v}=1.25 \times 21.5=26.875
\end{aligned}
$$




$$
\begin{aligned}
& A_{n v}=1.25 \times\left[21.5-3.5\left(1+\frac{1}{8}\right)\right]=21.95 \\
& A_{n t}=1.25 \times\left[2.5-0.5\left(1+\frac{1}{8}\right)\right]=2.4167 \\
& \varnothing R_{n}=0.6(65)(21.95)+1.0(65)(2.4167)=1013.1355
\end{aligned}
$$

Upper Limit

$0.6 F_{y} A_{g v}+U_{b s} F_{u} A_{n t}=0.6 \times 50 \times 26.875+1.0 \times 65 \times 2.4167=963.4$

$\emptyset R_{n}=0.75 \times 963.4=722.5$

Tension From M

$M=n \times r_{t} \times d$

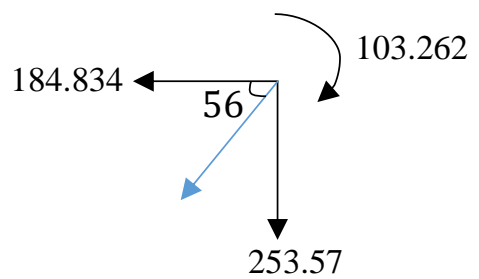

$r_{t}=2.1513$
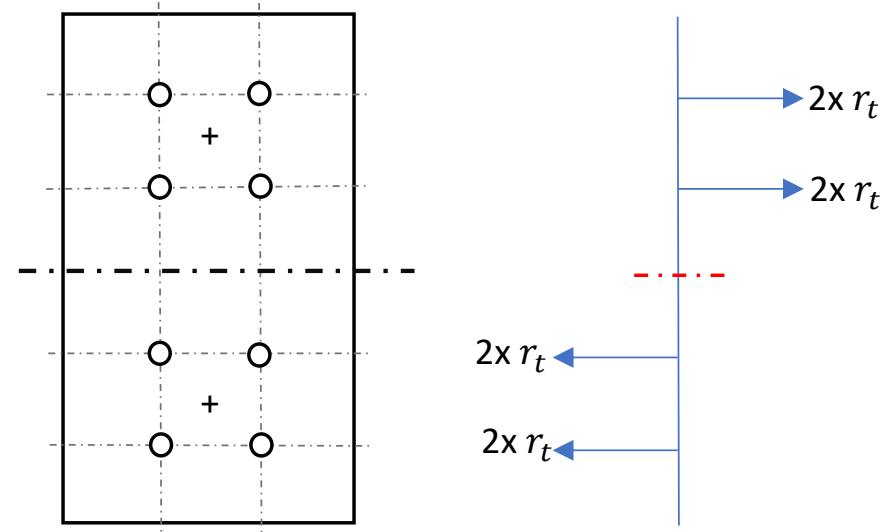

Shear $/$ bolt $=184.834 / 8=23.1$

Direct tensile load $/$ bolt $=253.57 / 8=31.7$

Total tensile load/bolt $=31.7+2.1513=33.852$

$$
\begin{aligned}
& F_{r v}=\frac{23.10}{0.785}=29.426 \\
& F_{n t}^{\prime}=1.3 F_{n t}-\frac{113}{0.75 \times 68}(29.5)=81.537<113
\end{aligned}
$$


Use $F_{n t}^{\prime}=81.537$

$\emptyset R_{n}=0.75(81.537)(0.7854)=48.03>34.578$

Prying action

Required $(t)=\sqrt{\frac{4 \times T \times b^{\prime}}{\emptyset_{b} \times p \times F_{u}(1+\delta \alpha)}}$

$W 10 \times 68\left[d=10.4, t_{w}=0.47, b_{f}=10.1, t_{f}=0.77\right]$

$\mathrm{M}=124$

$F=\frac{M}{10.4}=\frac{103.262 \times 12}{10.4}=119.15$

$P=F+264=119.15+253.57=372.72$

$b^{\prime}=b-\frac{d}{2}$

$b=\frac{\left(6-t_{w}\right)}{2}=\frac{(6-0.47)}{2}=2.765$

$b^{\prime}=2.765-\frac{1}{2}=2.265$

$a=2.125$

$a^{\prime}=a+\frac{d}{2}=2.1+\frac{1}{2}=2.6$

$d^{\prime}=1+\frac{1}{8}=1 \frac{1}{8}=1.125$

$p<2 b=2 \times 2.765=5.53 \quad$ use $p=s=5$

$\delta=1-\frac{d^{\prime}}{p}=1-\frac{1.125}{5}=0.775$

The design strength of one bolt is:-

$B=\emptyset \times F_{t} \times A_{b}=0.75(113)(0.785)=66.53$

$T=P=372.72$

Tension/bolts $=\frac{372.72}{8}=46.59 \quad \rightarrow T_{\text {bolt }}=46.59$

compute $\alpha$ 


$$
\begin{aligned}
& \frac{\beta}{T}-1=\frac{66.53}{46.59}-1=0.428 \\
& \alpha=\frac{0.428\left(\frac{2.6}{2.265}\right)}{0.775\left[1-0.3077\left(\frac{2.6}{2.265}\right)\right]}=\frac{0.4913}{0.5012}=0.98
\end{aligned}
$$

Required $(t)=\sqrt{\frac{4 \times T \times b^{\prime}}{\emptyset_{b} \times p \times F_{u}(1+\delta \alpha)}}$

Required $(t)=\sqrt{\frac{4 \times 46.59 \times 2.265}{0.9 \times 5 \times 65(1+0.775(0.98)}}=0.905 \rightarrow$ use $1.25^{\prime \prime}$

Link stiffeners for I-shaped cross sections (according to AISC seismic provisions)

Full depth web stiffeners must be provided on both sides of the link web at the diagonal brace ends of the link.

$b_{f}=$ link flange thickness,$t_{w}=$ link web thickness

stiffeners width $\geq b_{f}-2 t_{w}$

stiffeners thickness $\geq 0.75 t_{w}$ or $3 / 8$

Links of lengths $1.6 \mathrm{M}_{\mathrm{p}} / \mathrm{V}_{\mathrm{p}}$ or less

Web stiffeners spaced at intervals

$$
\leq 30 t_{w}-d / 5 \quad \text { link rotation angle } 0.08 \mathrm{rad} \text {. }
$$




\section{$W 16 \times 67$}

\section{Calculate the shear strength (AISC Seismic Provision,2016)}

$V_{p}=0.6 f_{y} t_{w} A_{w}=0.6 \times 50 \times 0.395 \times(16.3-2 \times 0.665-5.75)=109.3$

Shear strength (Steel Design Guide Series 2, Steel and Composite Beams with Web Openings, 2003

$F_{y}=50 k s i$

$W 16 \times 67\left(d=16.3, t_{w}=0.395, b_{f}=10.2, t_{f}=0.665\right)$

$V_{m}=V_{m b}+V_{m t}$

$V_{m b}=V_{p b} \times \alpha_{v b}$

$V_{m t}=V_{p t} \times \alpha_{v t}$

$\alpha_{v}=\frac{\sqrt{6}+\mu}{v+\sqrt{3}} \leq 1.0$

Top tee

$$
\begin{aligned}
& \mu=\frac{2 p_{r} d_{r}}{V_{p} S}=\frac{2(0) d_{r}}{V_{p} S}=0 \quad\left(p_{r}=A_{r} f_{y}\right), A_{r}=0 \\
& \alpha_{v t}=\frac{\sqrt{6}+\mu}{v+\sqrt{3}} \leq 1 \\
& v_{t}=\frac{a_{o}}{S_{t}}=\frac{27.1875}{1.165}=23.34 \\
& \alpha_{v t}=\frac{\sqrt{6}+0}{23.34+\sqrt{3}}=0.0977 \\
& V_{p t}=\frac{f_{y} t_{w} S}{\sqrt{3}}=\frac{50(0.395)(0.5+0.665)}{\sqrt{3}}=13.28 \\
& V_{m t}=\alpha_{v} V_{p t}=0.0977(13.28)=1.2975
\end{aligned}
$$

Bottom tee

$$
\begin{aligned}
& S_{b}=8.72+0.665=9.385 \\
& \alpha_{v b}=\frac{\sqrt{6}+\mu}{v+\sqrt{3}} \leq 1.0
\end{aligned}
$$




$$
\begin{aligned}
& \mu=\frac{2 p_{r} d_{r}}{V_{p} S}=\frac{2(0) d_{r}}{V_{p} S}=0 \\
& \left(p_{r}=A_{r} f_{y}\right) \leq \frac{f_{y} t_{w} a_{o}}{2 \sqrt{3}}=\frac{50 \times 0.395 \times 27.8}{2 \sqrt{3}}=155 \\
& A_{r}=2[0.75 \times 5.0]=7.5 \\
& S^{\prime}=S-\frac{A_{r}}{2 b_{f}}=9.6875-\frac{7.5}{2(10.2)}=9.32 \\
& P_{r}=f_{y} A_{r}=50(7.5)=375 \\
& \frac{f_{y} t_{w} a_{o}}{2 \sqrt{3}}=170.5 \\
& \mu=\frac{2 p_{r} d_{r}}{V_{p} S}, \quad S=9.6875 \\
& V_{p b}=\frac{f_{y} t_{w} S}{\sqrt{3}}=\frac{50(0.395)(9.675)}{\sqrt{3}}=110.46 \\
& \mu=\frac{2 \times 155 \times 9.06}{110.46 \times 9.6875}=2.624 \\
& v=\frac{a_{o}}{S^{\prime}}=\frac{27.1875}{9.32}=2.917 \\
& \alpha_{v b}=\frac{\sqrt{6}+2.624}{2.917+\sqrt{3}}=1.09 \\
& V_{m b}=V_{p b} \times \alpha_{v b}=1.0(110.46)=110.46 \\
& V_{m}=110.46+0.36=110.82 \\
& V_{p}^{\prime}=\frac{f_{y} t_{w} d}{\sqrt{3}}=\frac{50 \times 0.395 \times 16.3}{\sqrt{3}}=223.2 \\
& V_{m}=110.82 \leq \frac{2}{3}\left(V_{p}^{\prime}\right)=148.86 \quad \rightarrow \text { okay } \\
& \hline
\end{aligned}
$$




$$
\begin{array}{rl}
Z=0.665 \times 1 & 0.2 \times\left(0.5227+0.25+5.75+0.25+\frac{0.665}{2}\right)+0.25 \times 0.395 \\
& \times\left(0.5227+0.25+5.75+\frac{0.25}{2}\right)+0.25 \times 0.395 \times\left(0.5227+\frac{0.25}{2}\right) \\
& +\left(0.5227 \times 10.395 \times \frac{0.5277}{2}\right)+10.395 \times 0.2273 \times \frac{0.2273}{2}+8 \\
& \times 0.395(4+0.2273)+10.2 \times 0.665\left(8+0.2273+\frac{0.665}{2}\right) \\
& =122.013 \mathrm{in}^{3}
\end{array}
$$$$
M_{p}=122.013 \times 50=6100 \text { kips. } \text { in }=508.4 \text { kips. } f t
$$

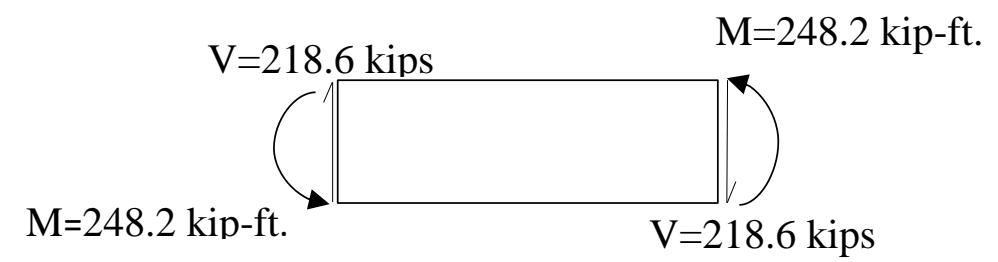

$\emptyset_{0}=2$

$V_{u}=\emptyset \mathrm{o} V_{p}=2 \times 109.3=218.6$ kips

$=\frac{V_{u} \times e}{2}=\frac{218.6 \times 27.25}{2 \times 12}=248.2$ kips. ft

$W 16 \times 67\left(d=16.3, t_{w}=0.395, b_{f}=10.2, t_{f}=0.665\right)$

$e_{o}=\frac{16.3}{2}-\frac{5.75}{2}-0.395-0.25=4.63$

$M_{p}=F_{y}\left(Z-\frac{h_{o}^{2} t_{w}}{4}-h_{o} e_{o} t_{w}+\frac{\left(t_{h s}\right)^{2}\left(b_{f}-t_{w}\right)}{4}\right)$

$M_{p}=50\left(130-\frac{5.75^{2} \times 0.395}{4}-5.75 \times 4.63 \times 0.395+\frac{(0.75)^{2} \times(10.2-0.395)}{4}\right)=$ 5880 kips. in $=490$ kips. $f t$

Design procedures are the same as of $\mathrm{W} 18 \times 76$ 


\section{Appendix B. Model Parameters}

The combined nonlinear isotropic/ kinematic hardening model used here was suggested by Lemaitre and Chaboche (1994). It is a combination of isotropic hardening, which defines the size of the yield surface, and kinematic hardening, which describes the translation of the yield surface through the backstress $\alpha$.

For the isotropic hardening model, the exponential law given in equation (B-1) shows the isotropic hardening expression included within Abaqus;

$\sigma^{o}=\sigma_{/ o}+Q_{\infty}\left(1-e^{-b \varepsilon^{p l}}\right)$

$Q_{\infty}$ is the max change in the size of the yield surface.

$\mathrm{b}$ defines the rate at which the size of the yield surface changes as plastic straining develops.

$\sigma_{/ o}$ is the initial yield stress.

$Q_{\infty}$ and $b$ are material parameters that should be obtained from cyclic test data

$\sigma^{o}$ is the size of the yield surface.

For linear kinematic hardening model, see expression (B-2);

$\alpha=C \frac{1}{\sigma^{o}}(\sigma-\alpha) \varepsilon^{\cdot p l}$

Where:

$\mathrm{C}$ defines as the initial kinematic hardening moduli 
$\alpha$ is the backstress tensor that describes the translation of the yield surface in stress space and defines the central position of the current yield surface.

$\varepsilon^{-. p l}$ is the equivalent plastic strain rate, $\varepsilon^{-. p l}=\int \sqrt{\frac{2}{3} d \epsilon_{i j}^{p} d \epsilon_{i j}^{p}}$ for isotropic Mises plasticity.

The math equation of nonlinear kinematic hardening is expressed in equation (B-3):

$\alpha_{k}=C_{k} \frac{1}{\sigma^{o}}(\sigma-\alpha) \varepsilon^{-. p l}-\gamma_{k} \alpha_{k} \varepsilon^{-. p l}$

The overall backstress is computed from the superposition of several kinematic hardening components, as shown in this relation: $\alpha=\sum_{k=1}^{N} \alpha_{k}$

Where $\mathrm{N}$ is the number of backstresses

Clearly, the effective plastic strain at any point should be calculated through integration over the complete history of deformation.

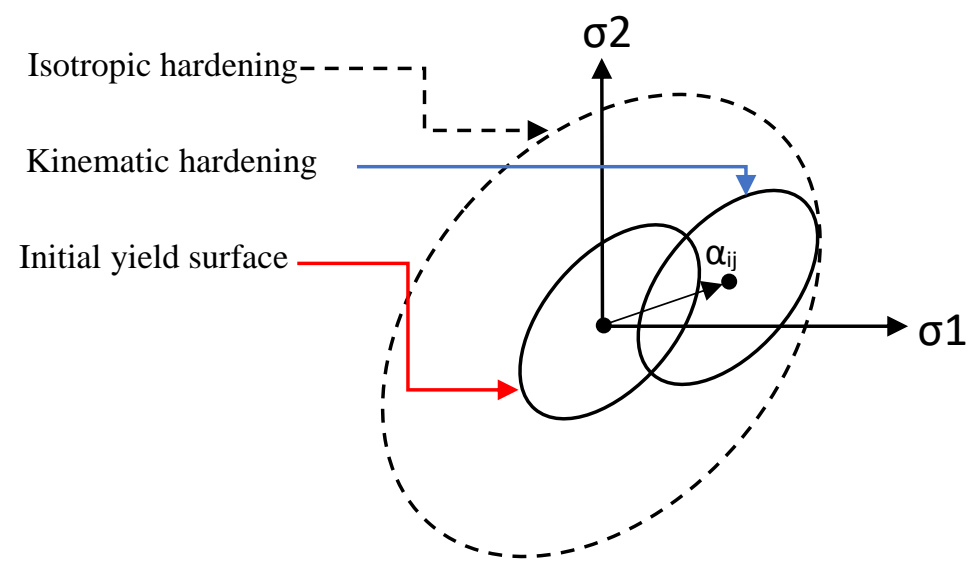


$\mathrm{C}$ and $\Upsilon$ are material parameters, which have to be obtained from a cyclic test, , and $\Upsilon_{\mathrm{k}}$ determine the rate at which the kinematic hardening moduli decrease with increasing plastic deformation. $\mathrm{K}^{\text {th }}$ is the backstress. If $\mathrm{C}_{\mathrm{k}}$ and $\Upsilon_{\mathrm{k}}$ are zero, then the hardening model is isotropic.

If $\Upsilon_{\mathrm{k}}=\mathrm{zero}$, then the law becomes a linear Ziegler hardening, $\alpha=C \frac{1}{\sigma^{o}}(\sigma-\alpha) \varepsilon^{\cdot p l}$ or linear kinematic hardening model 


\section{Appendix C. Design Drawings}

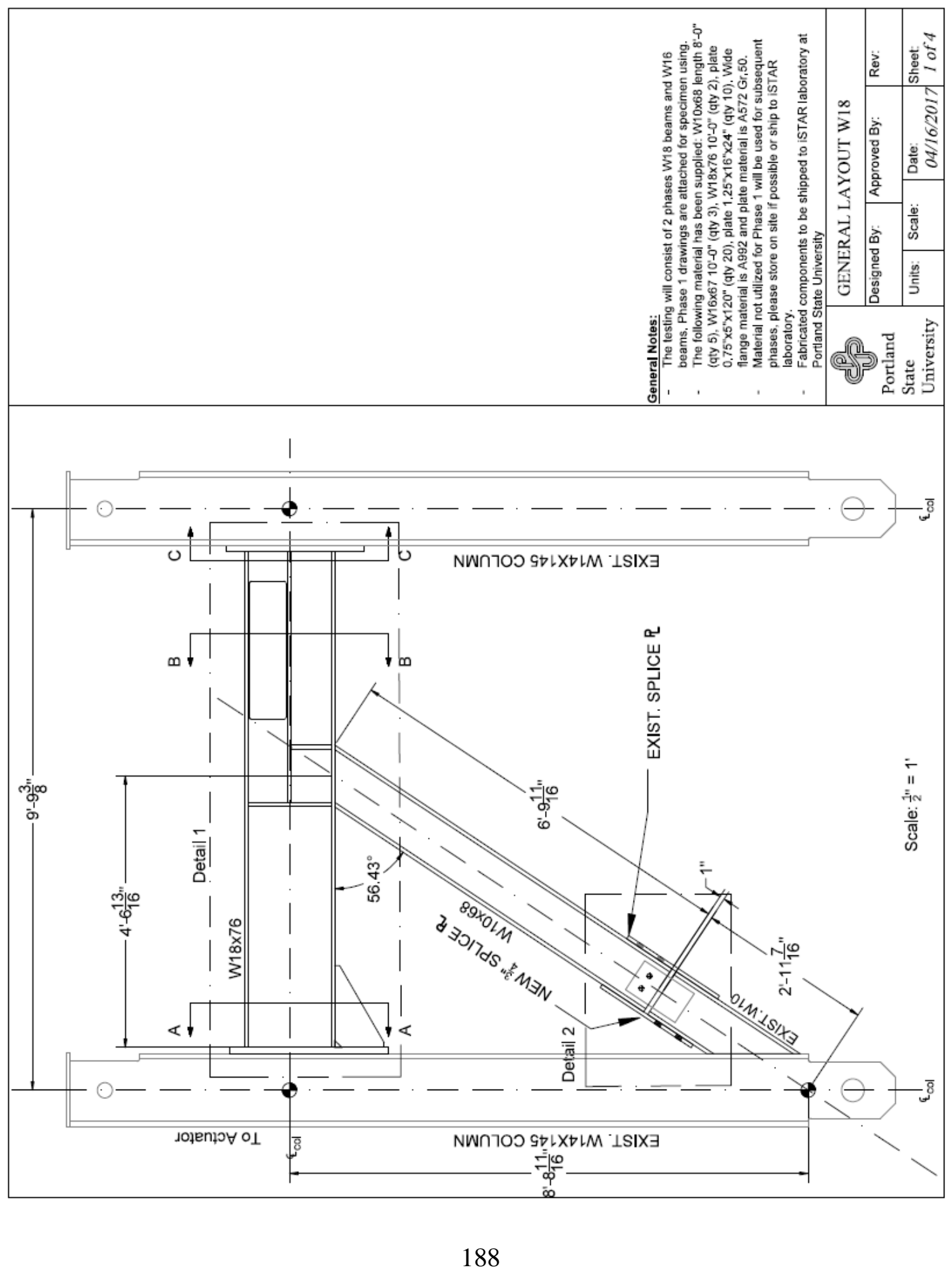




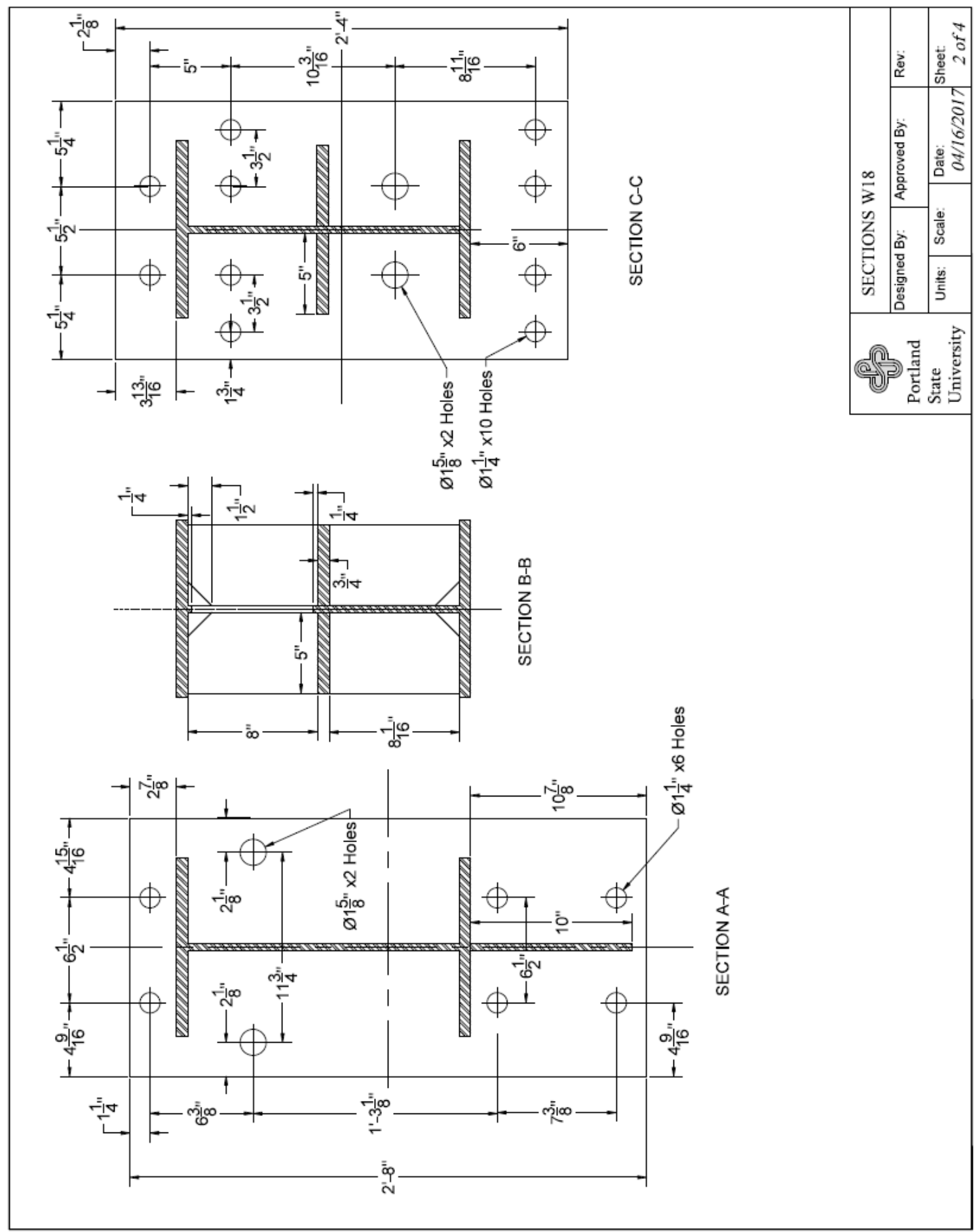




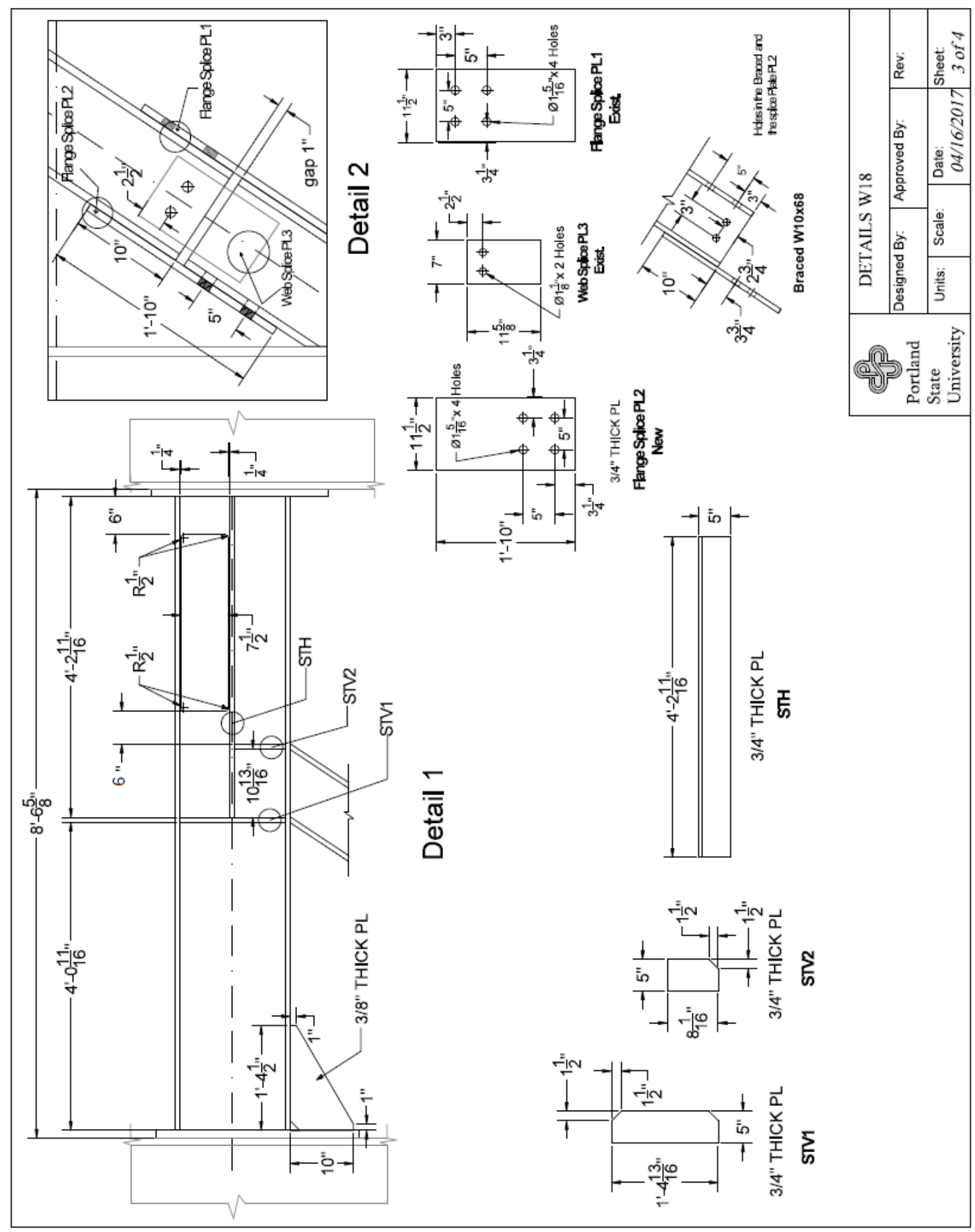




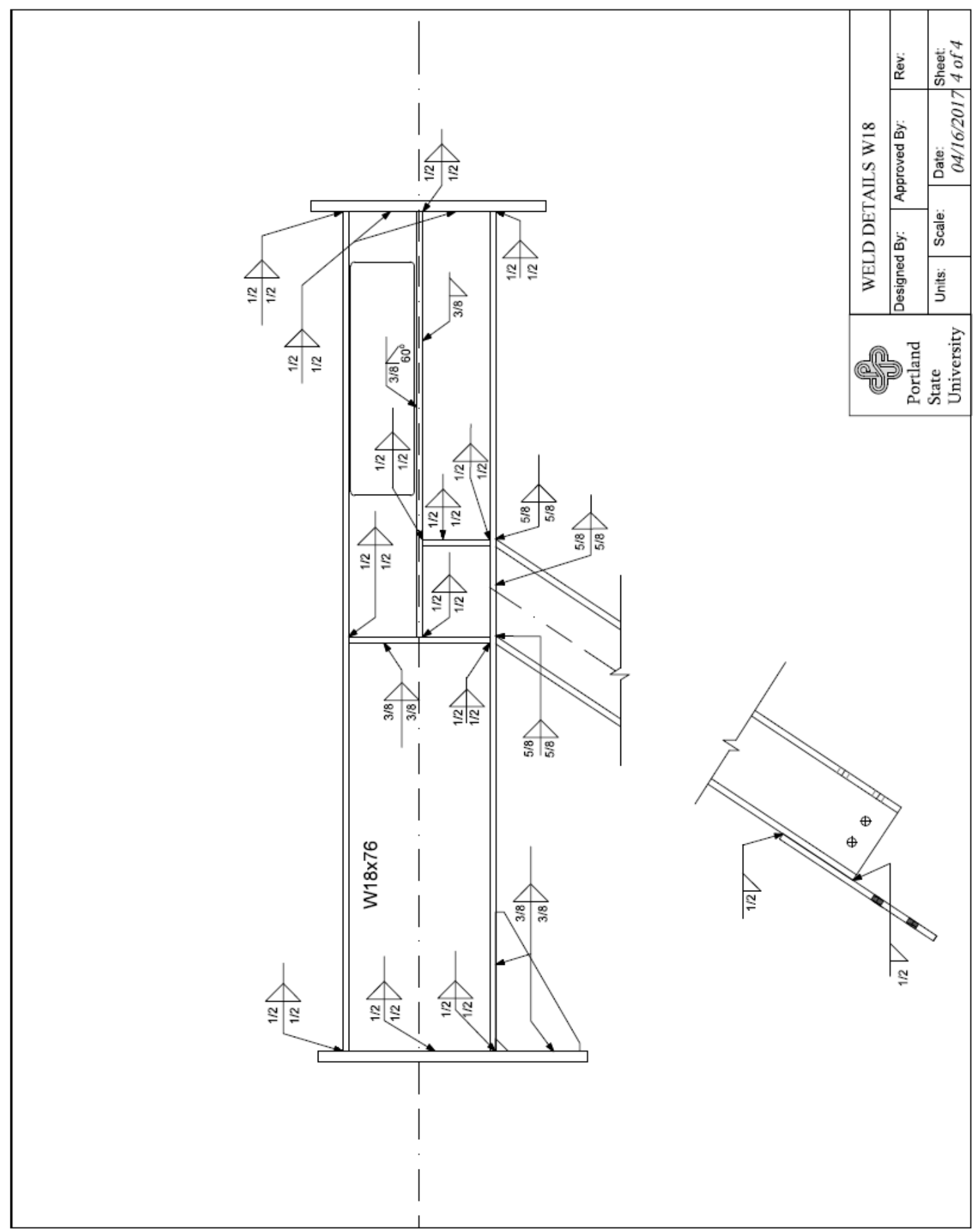




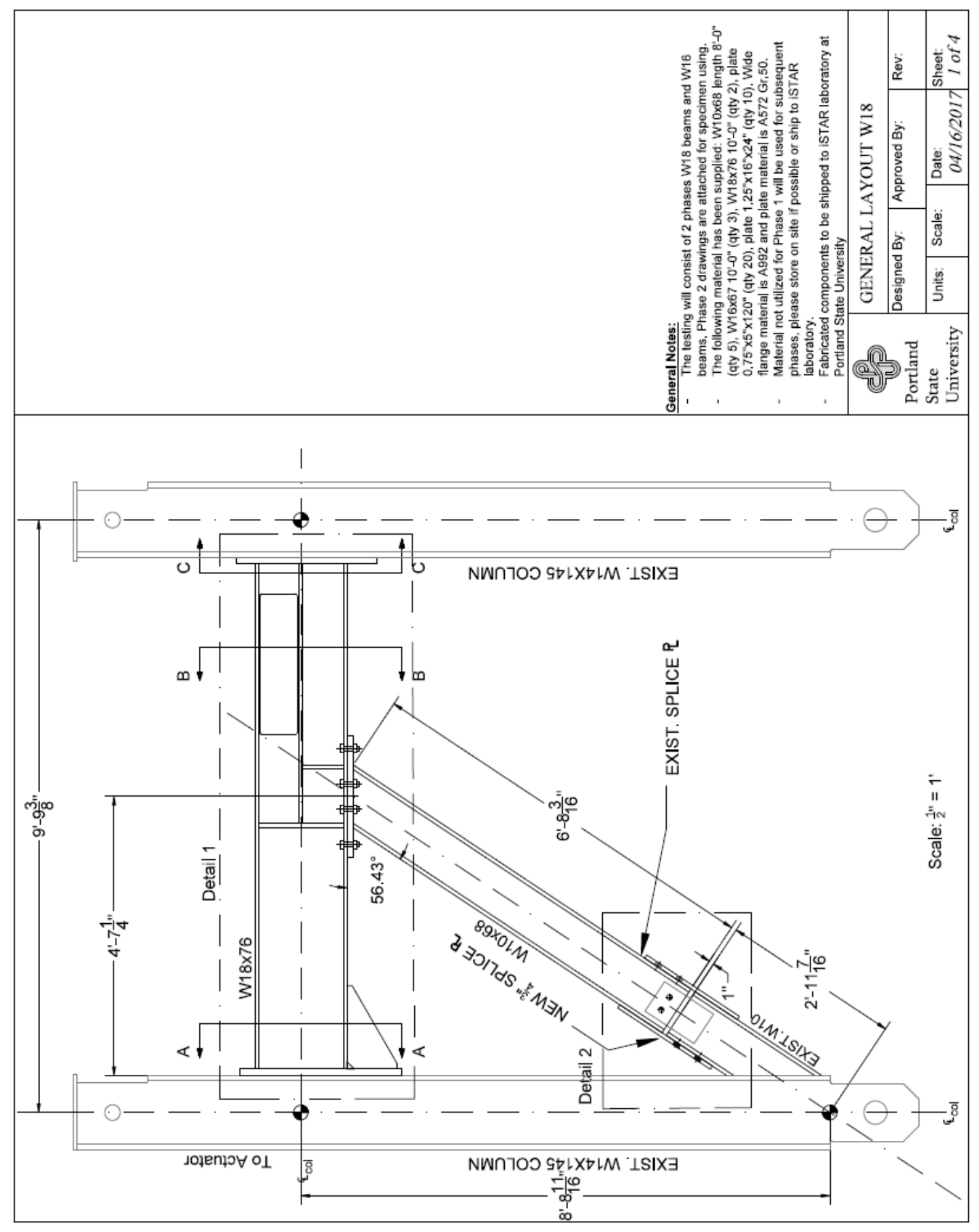




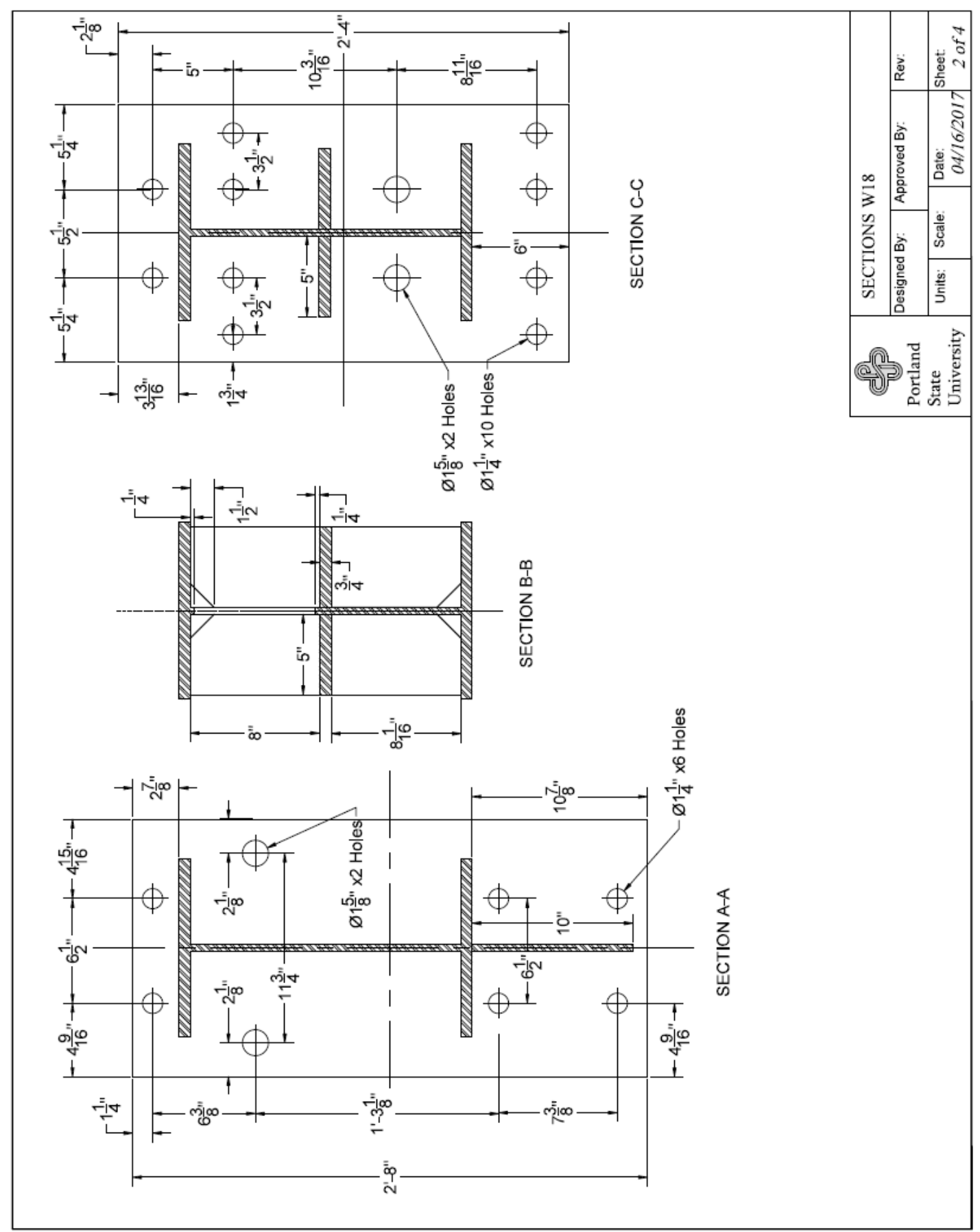




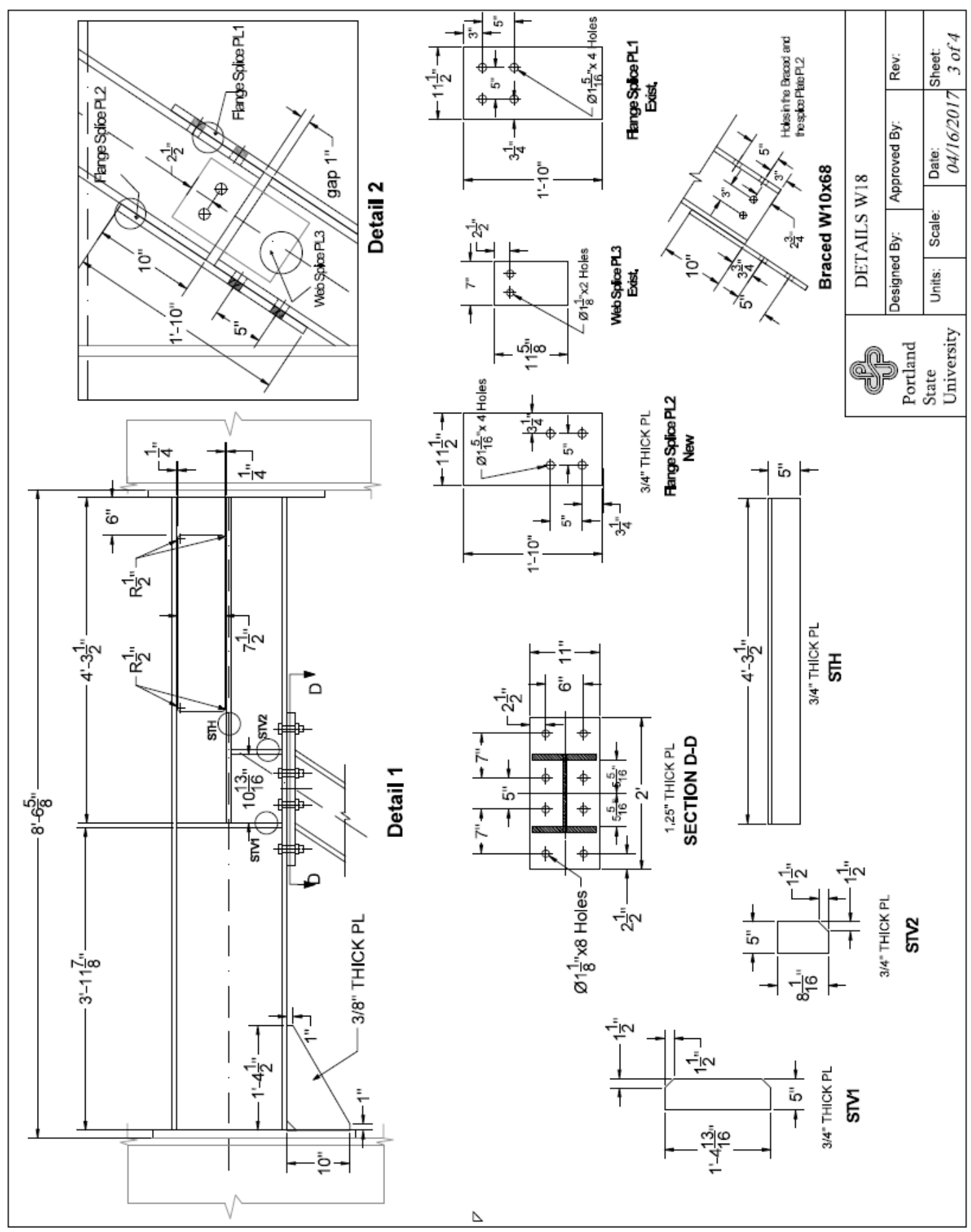




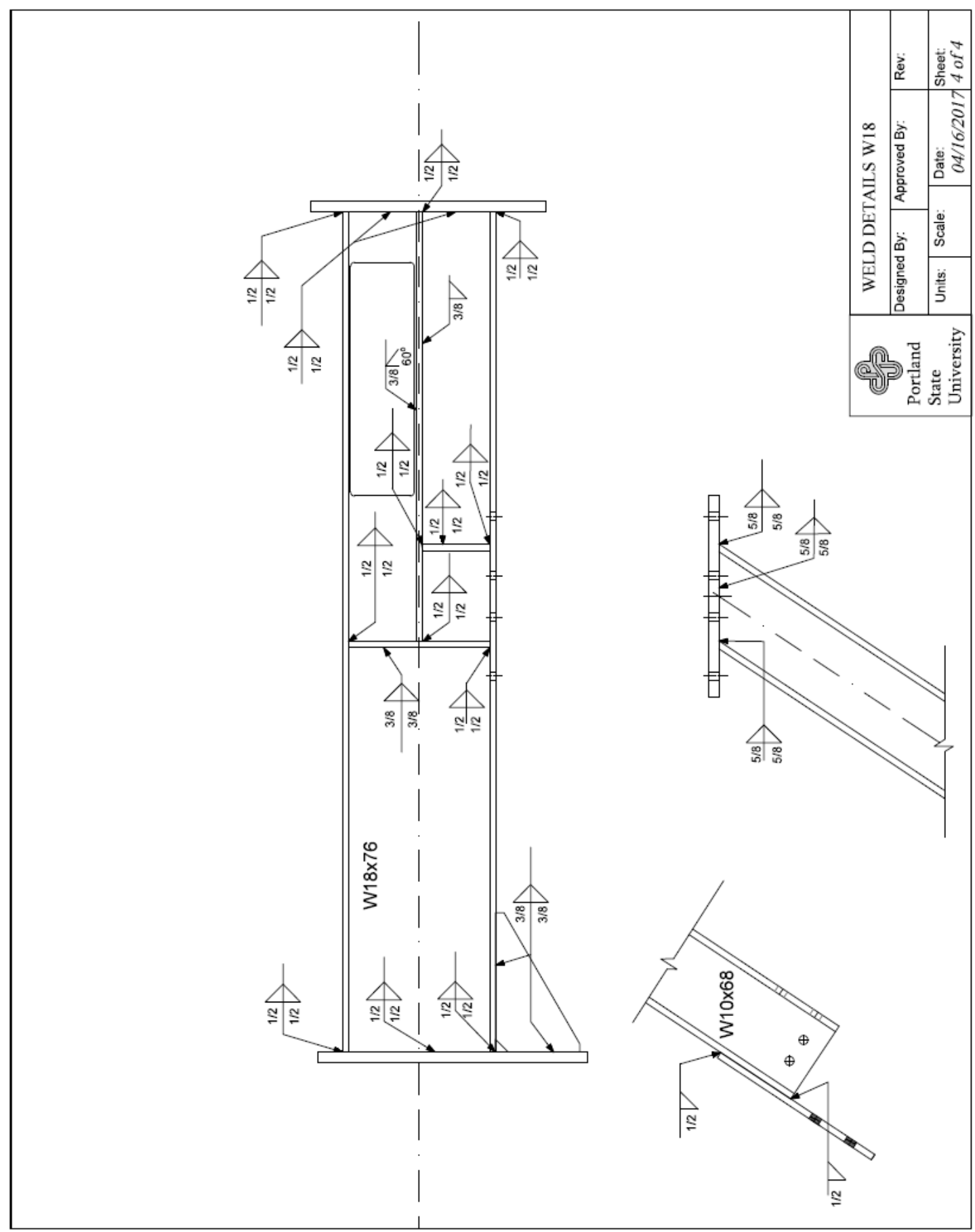




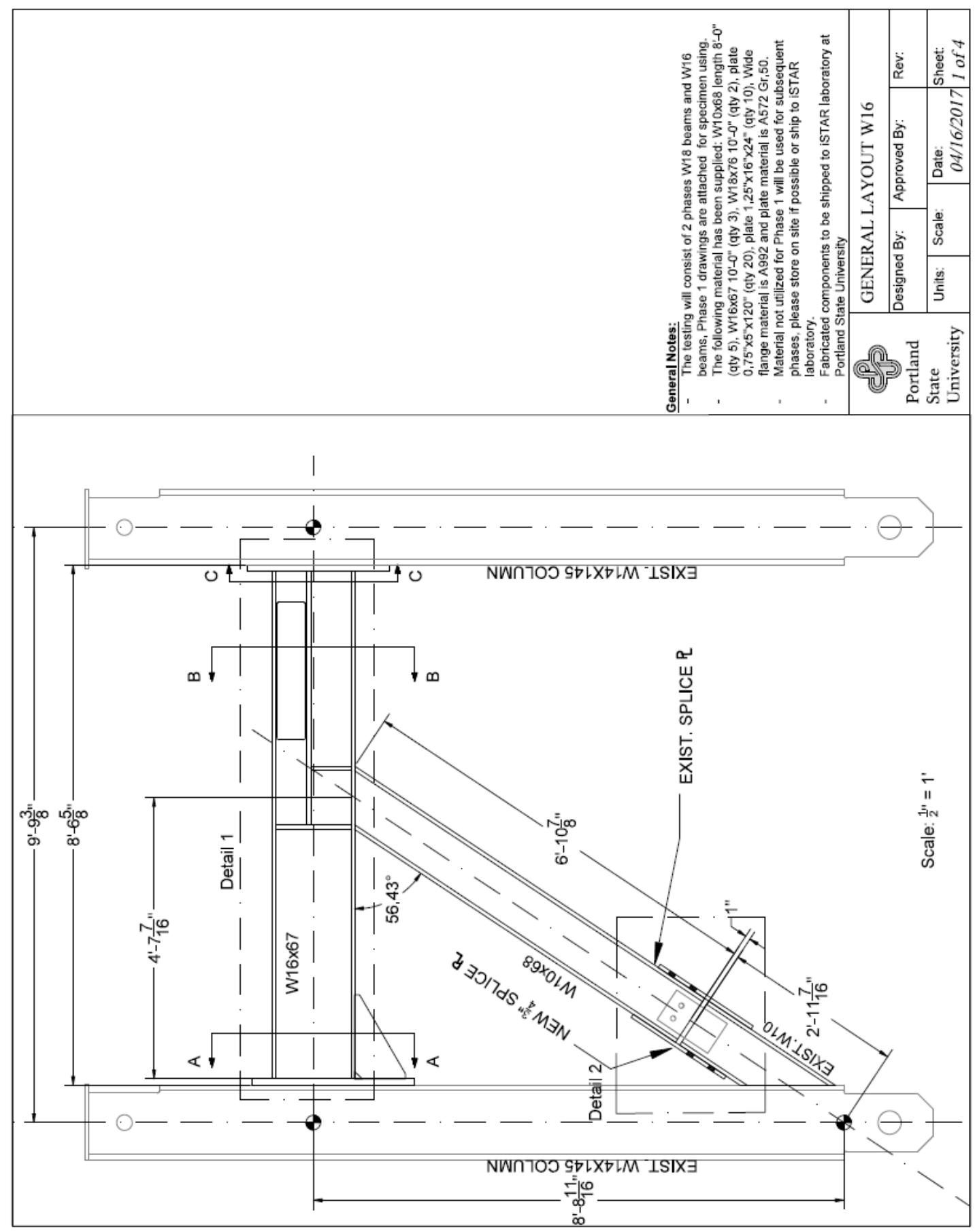




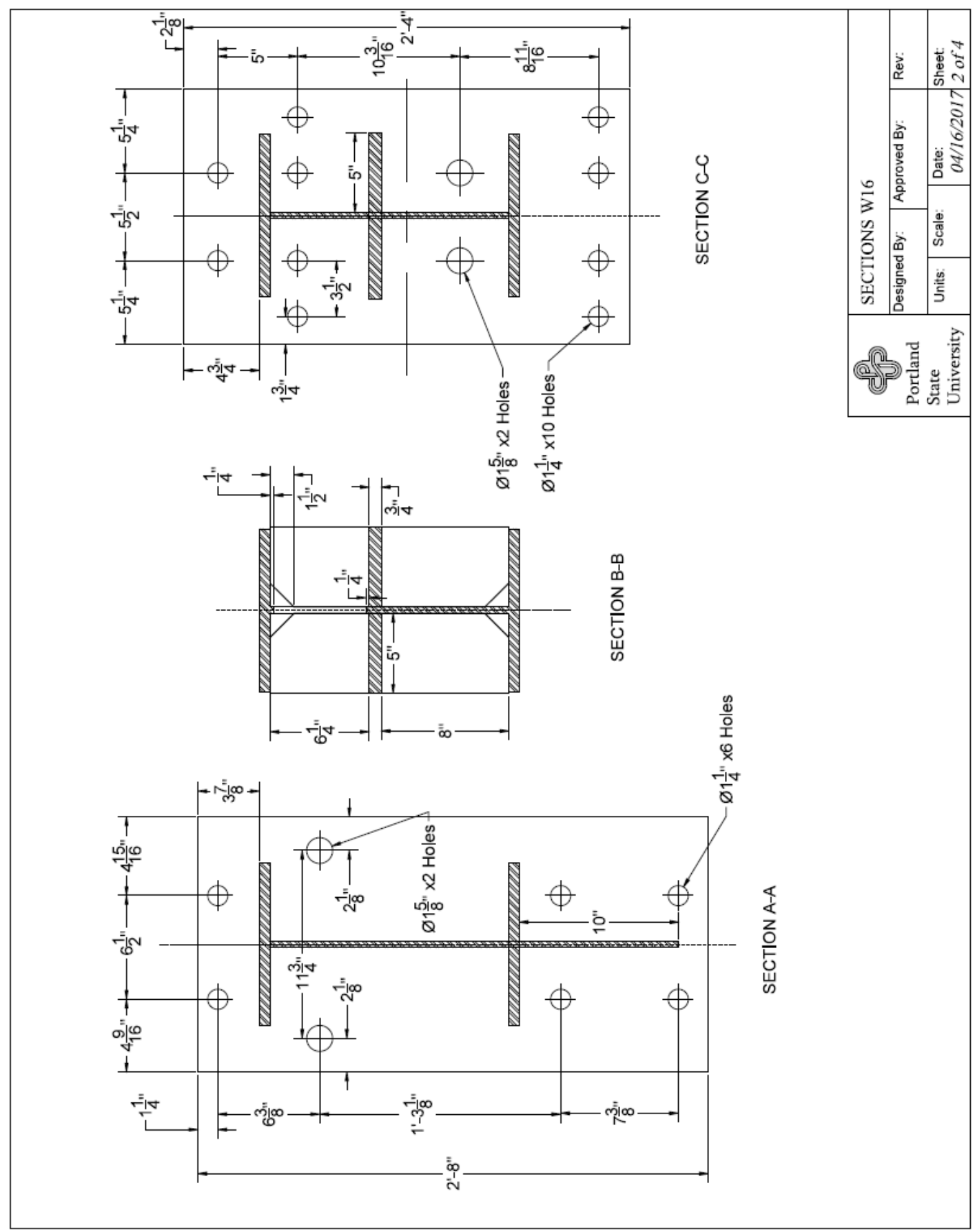




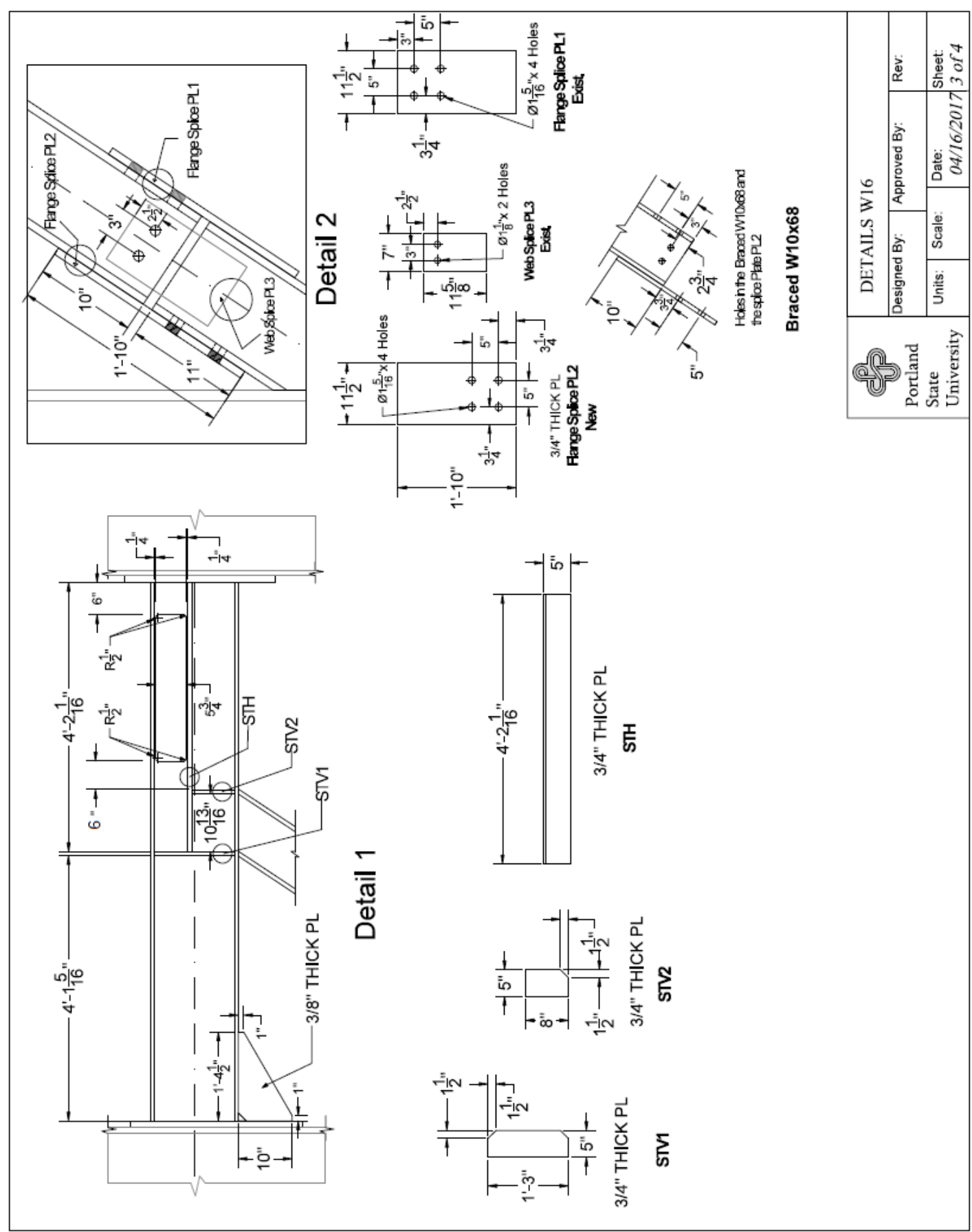




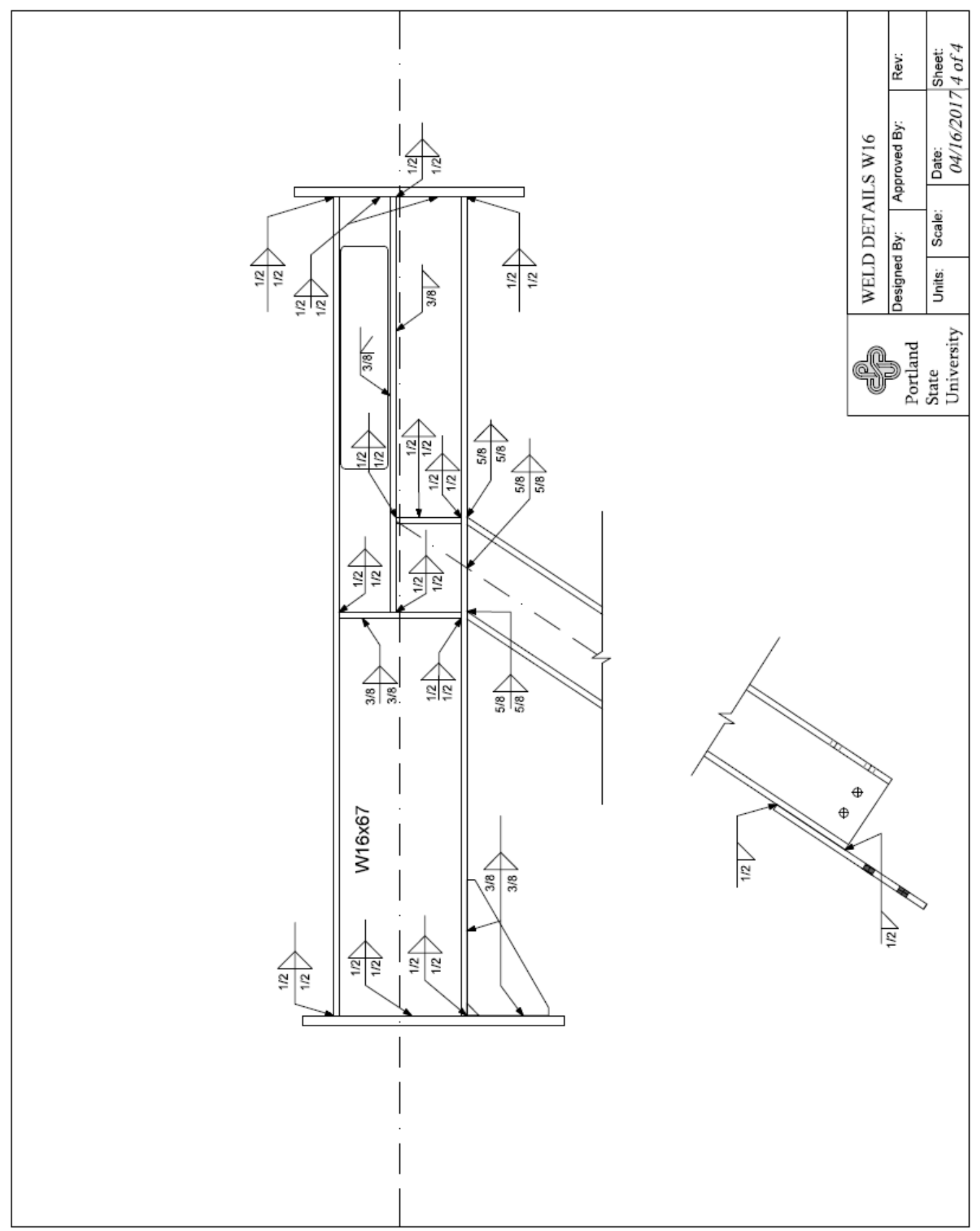




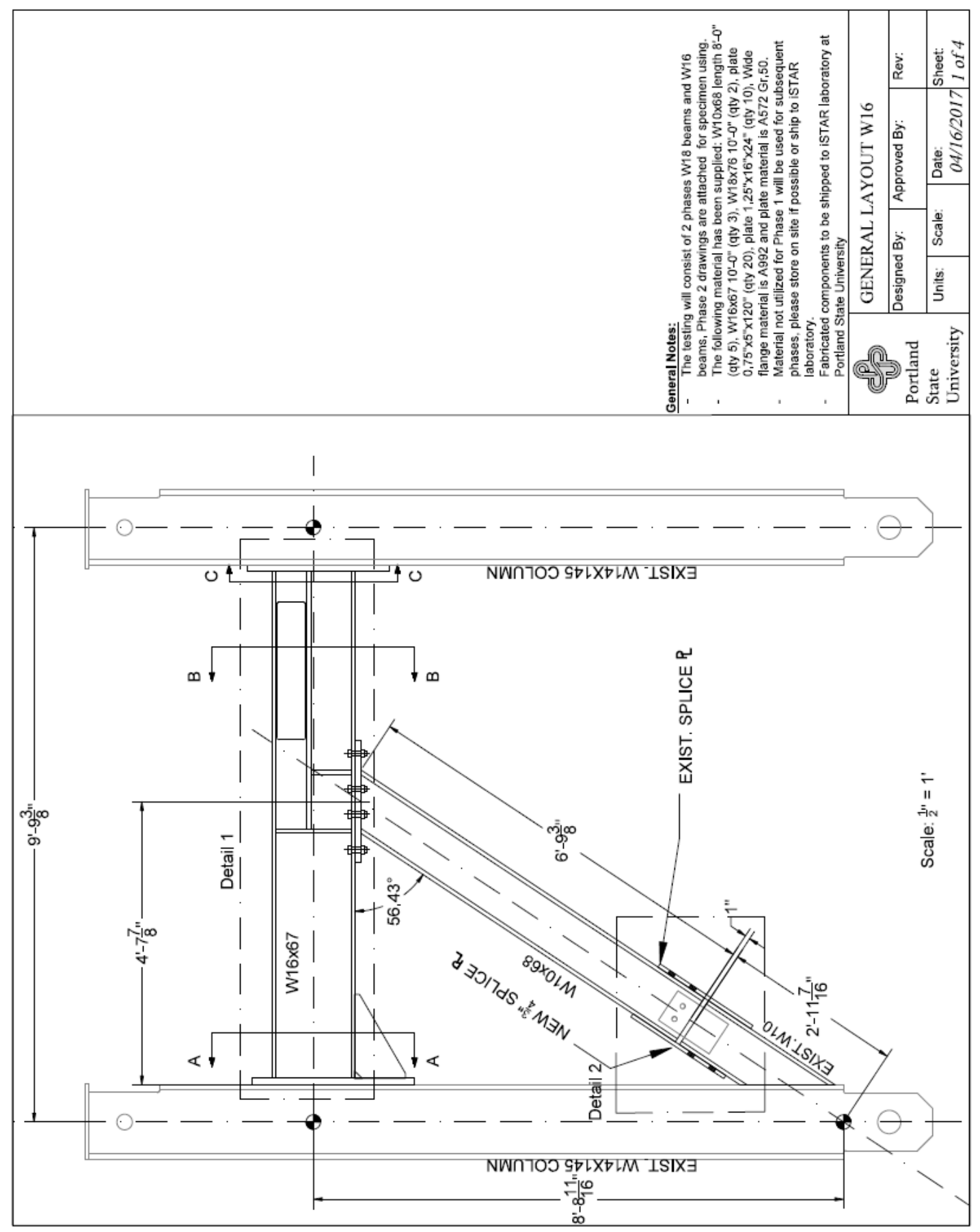




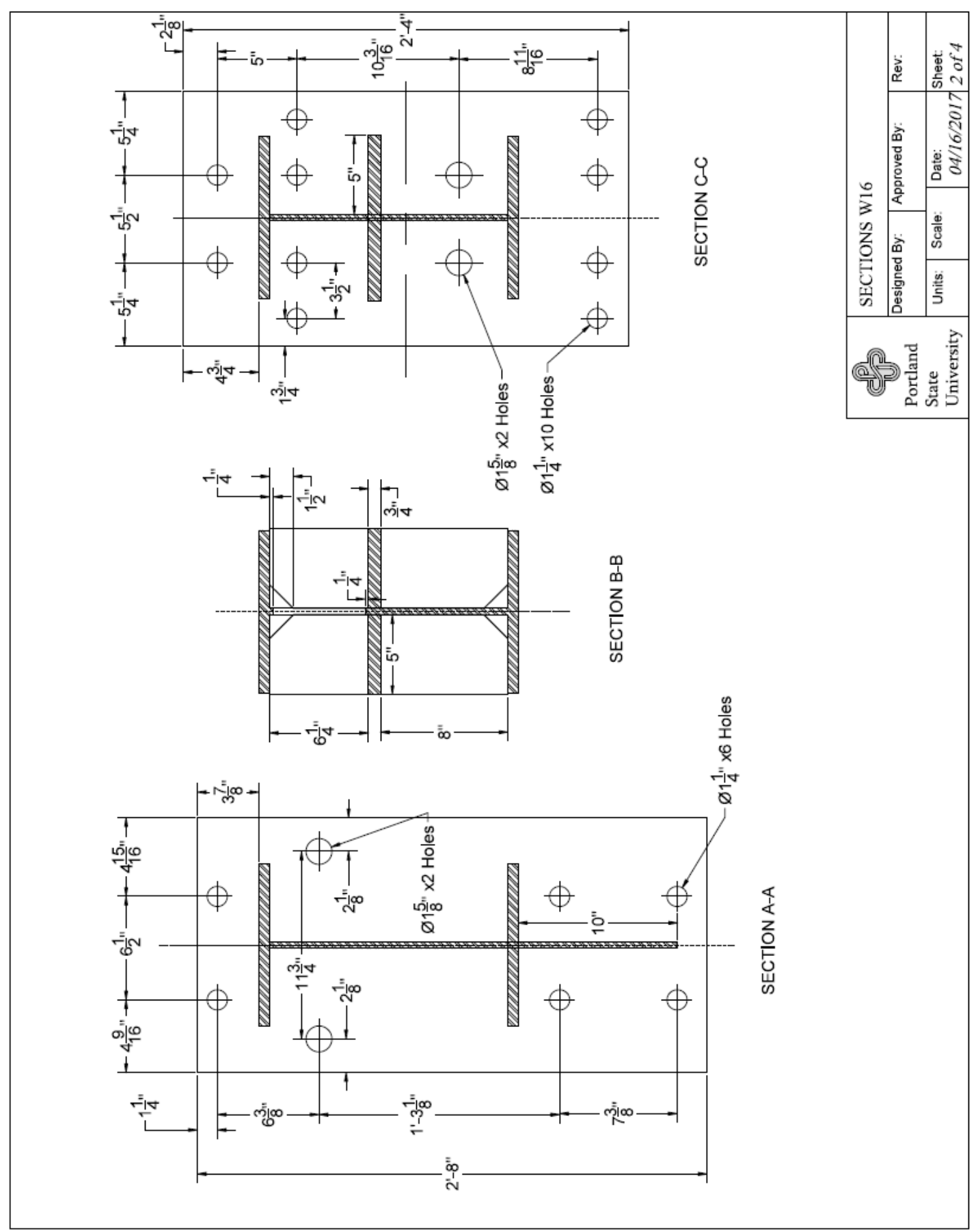




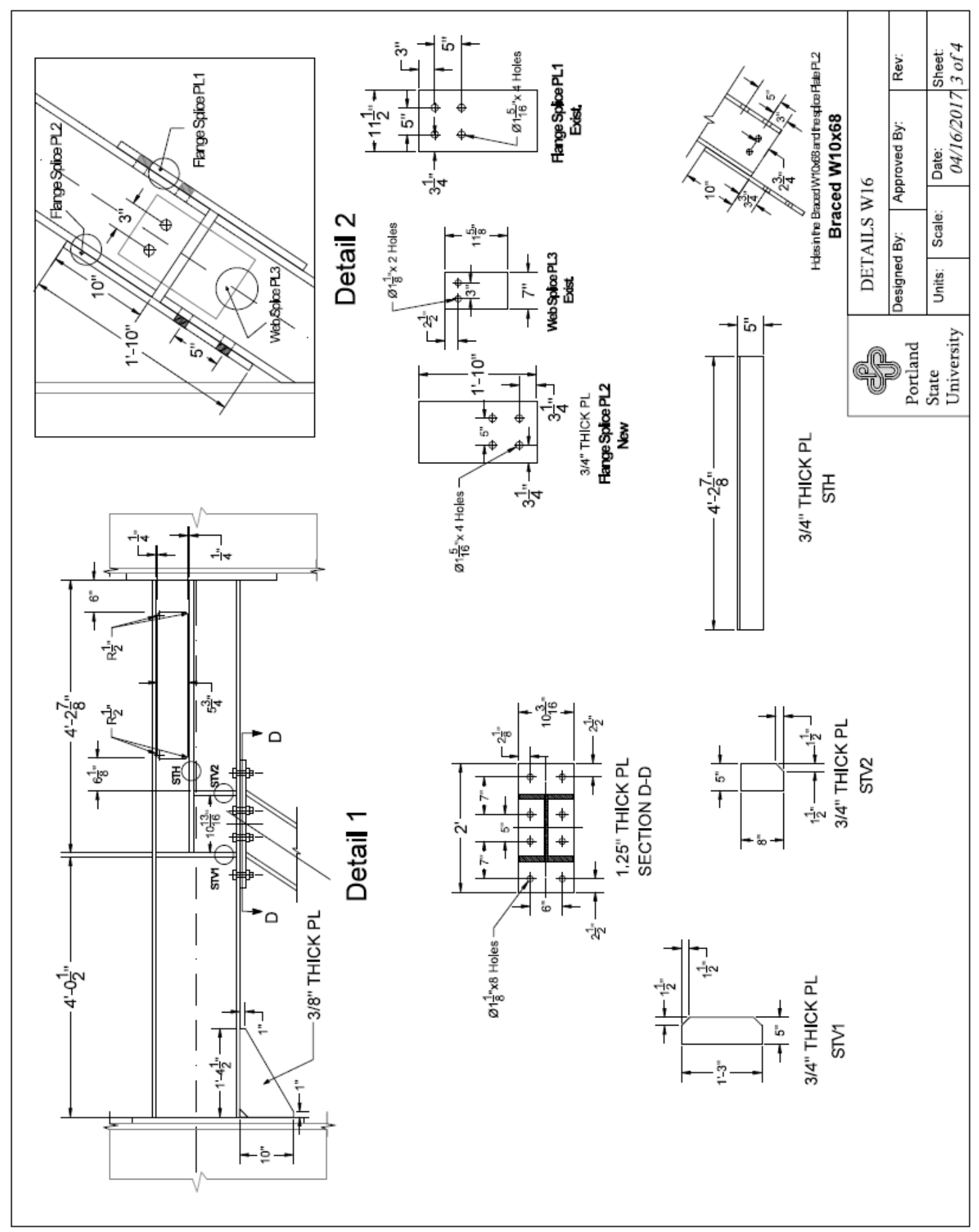




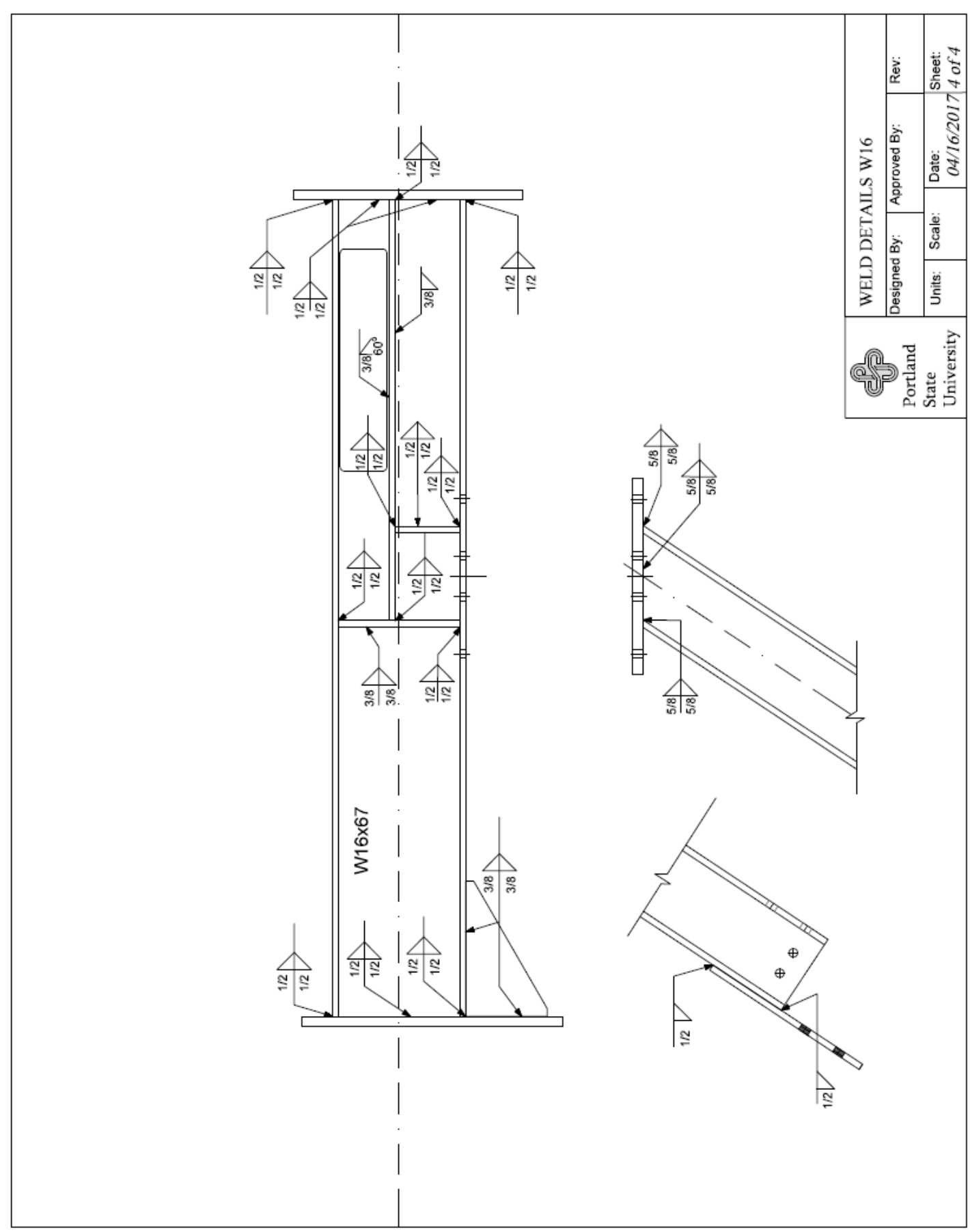




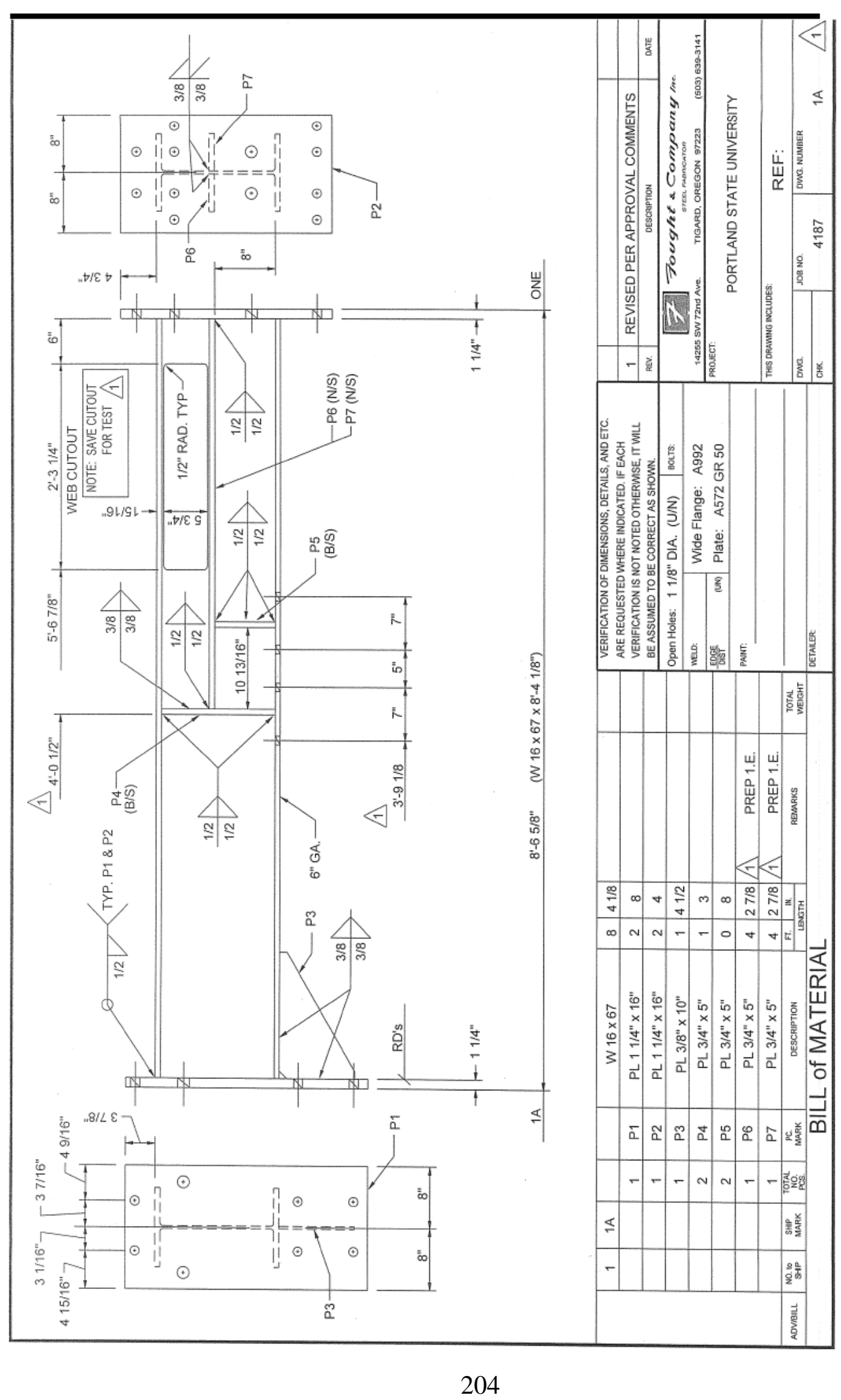




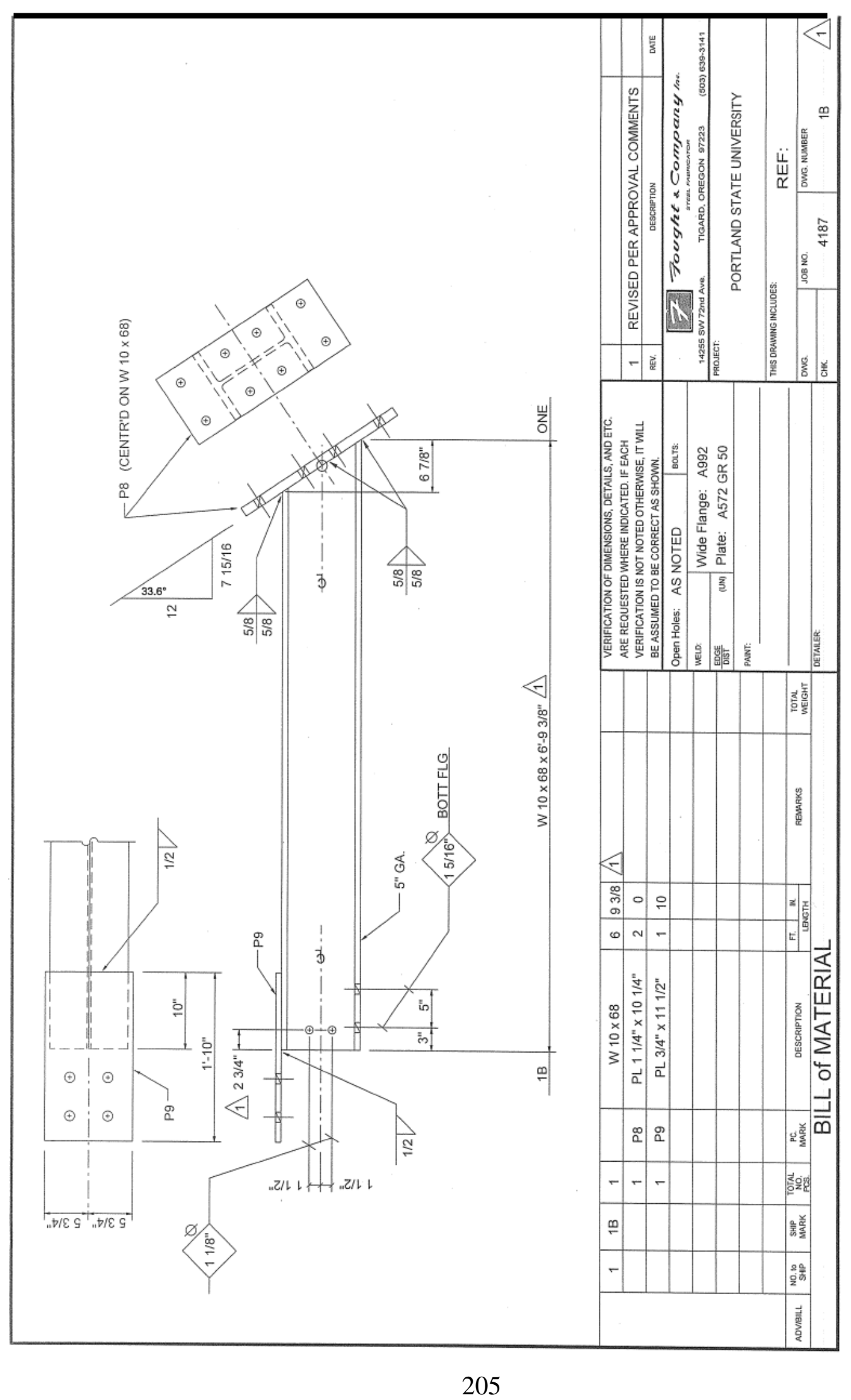




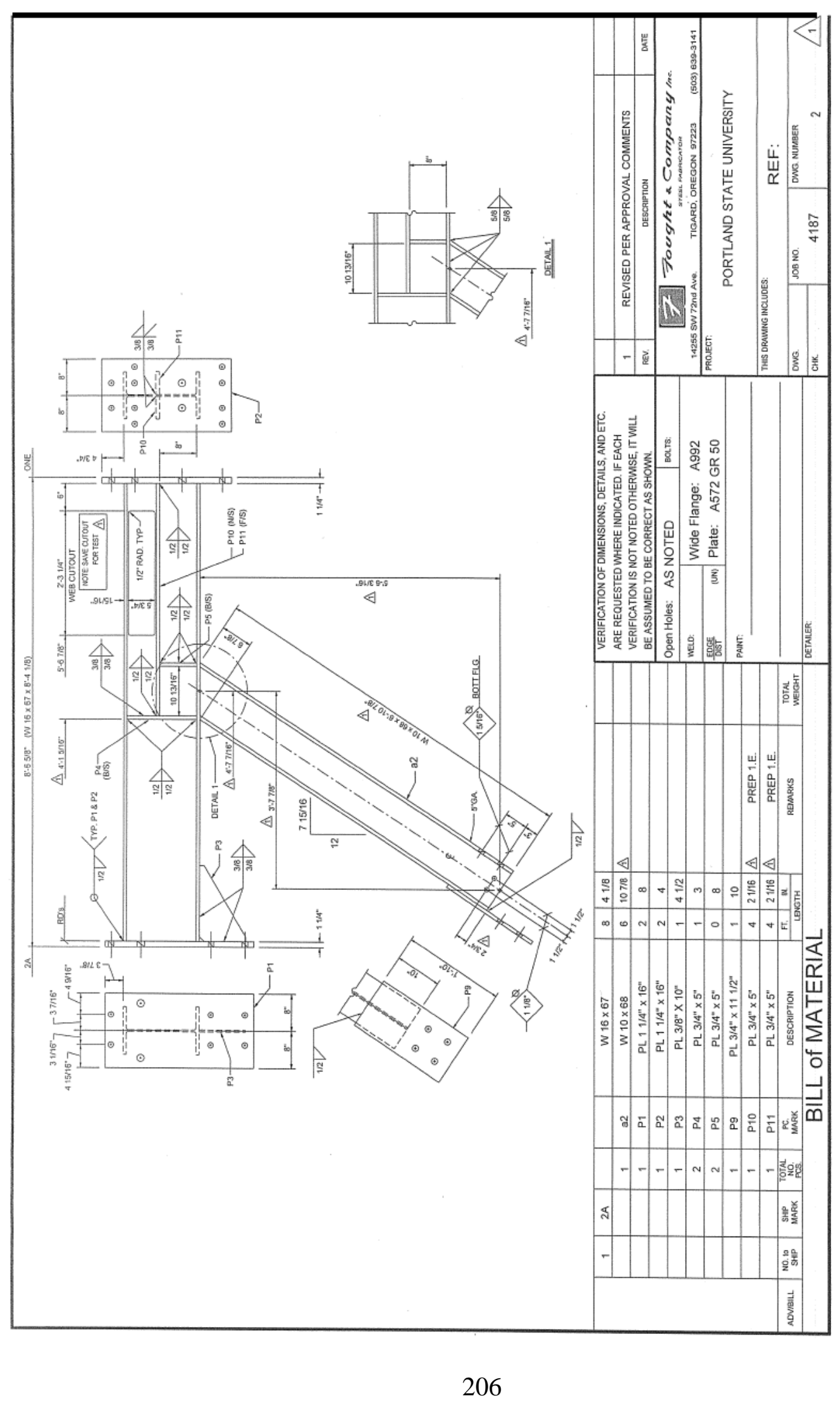




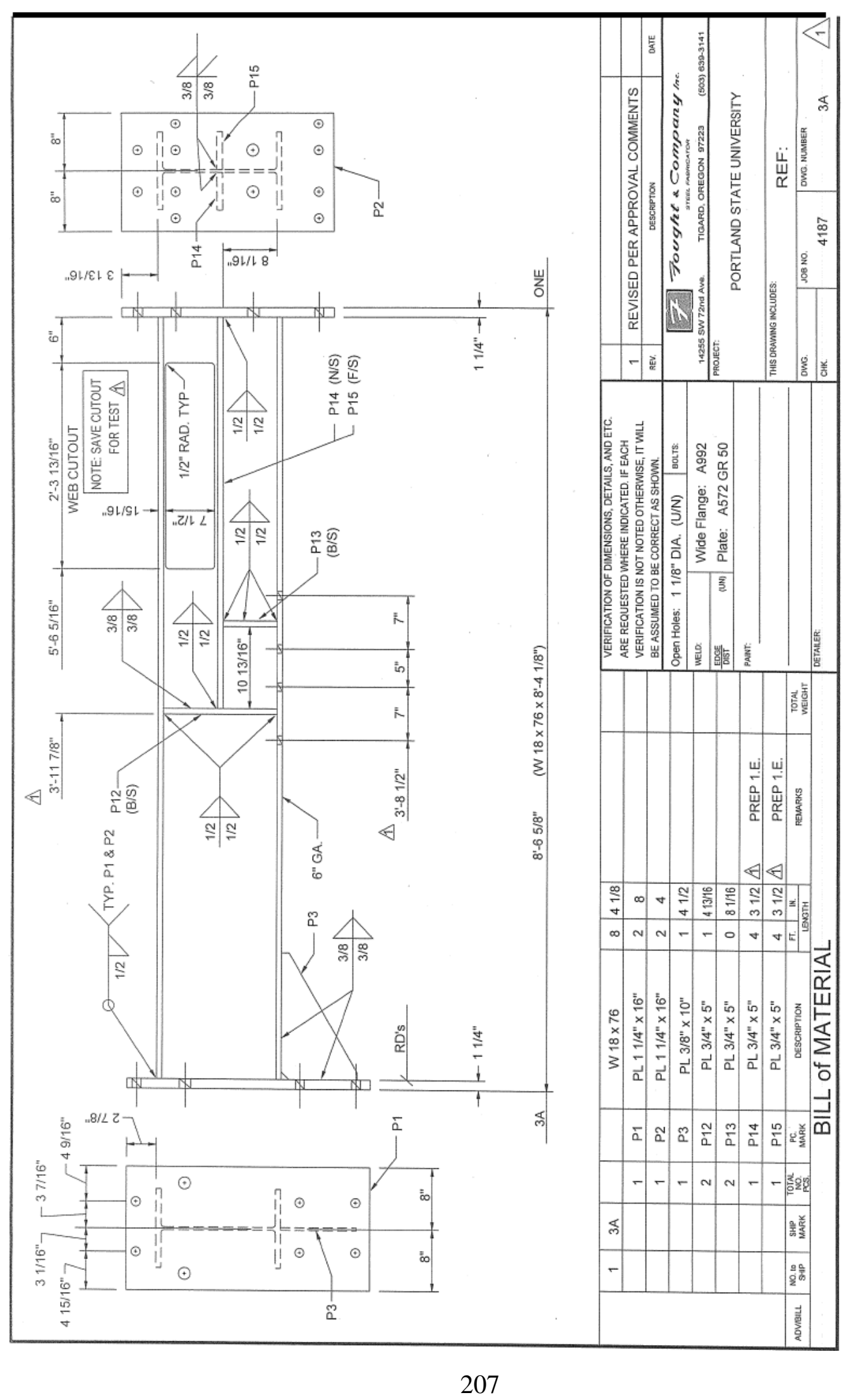




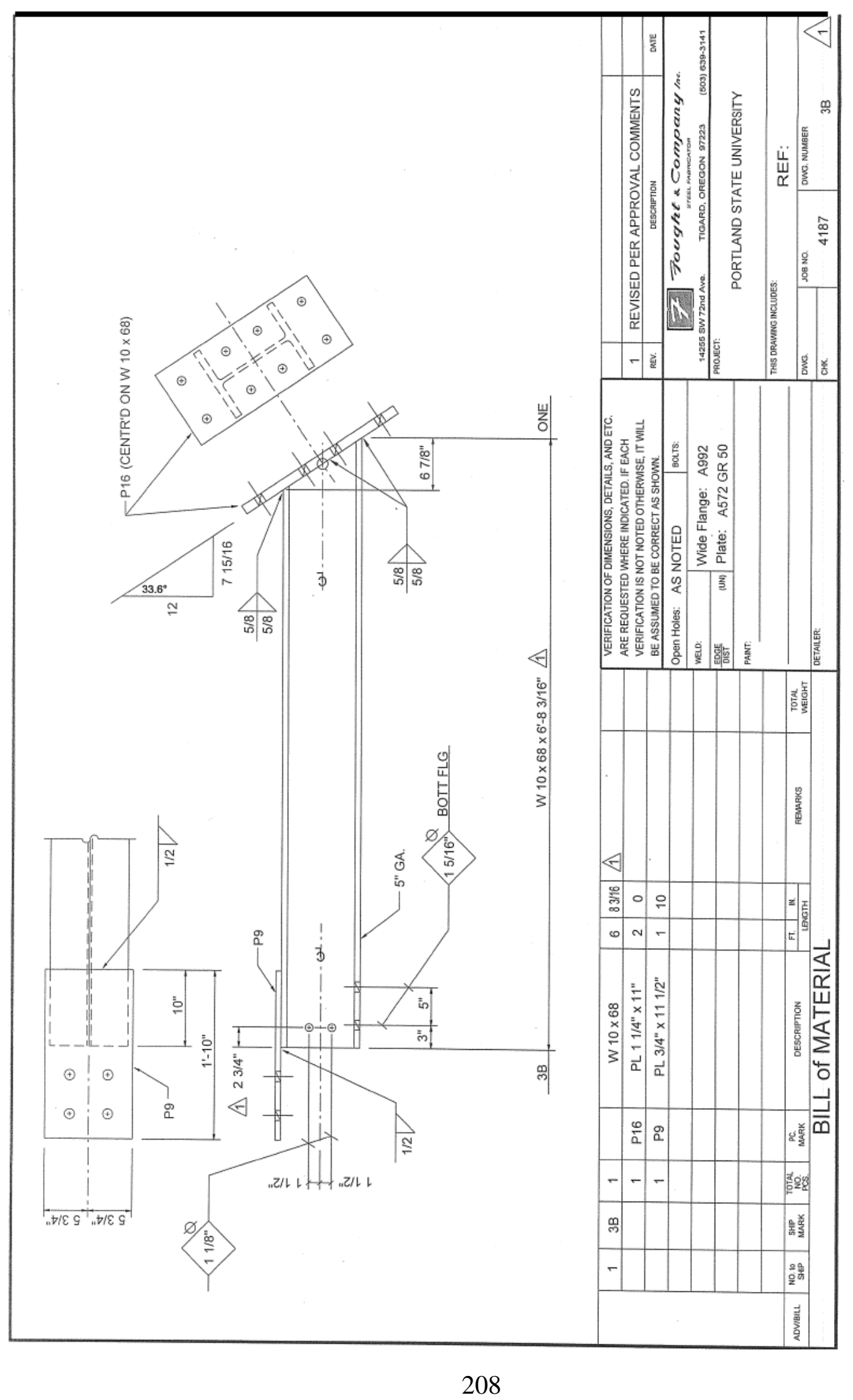




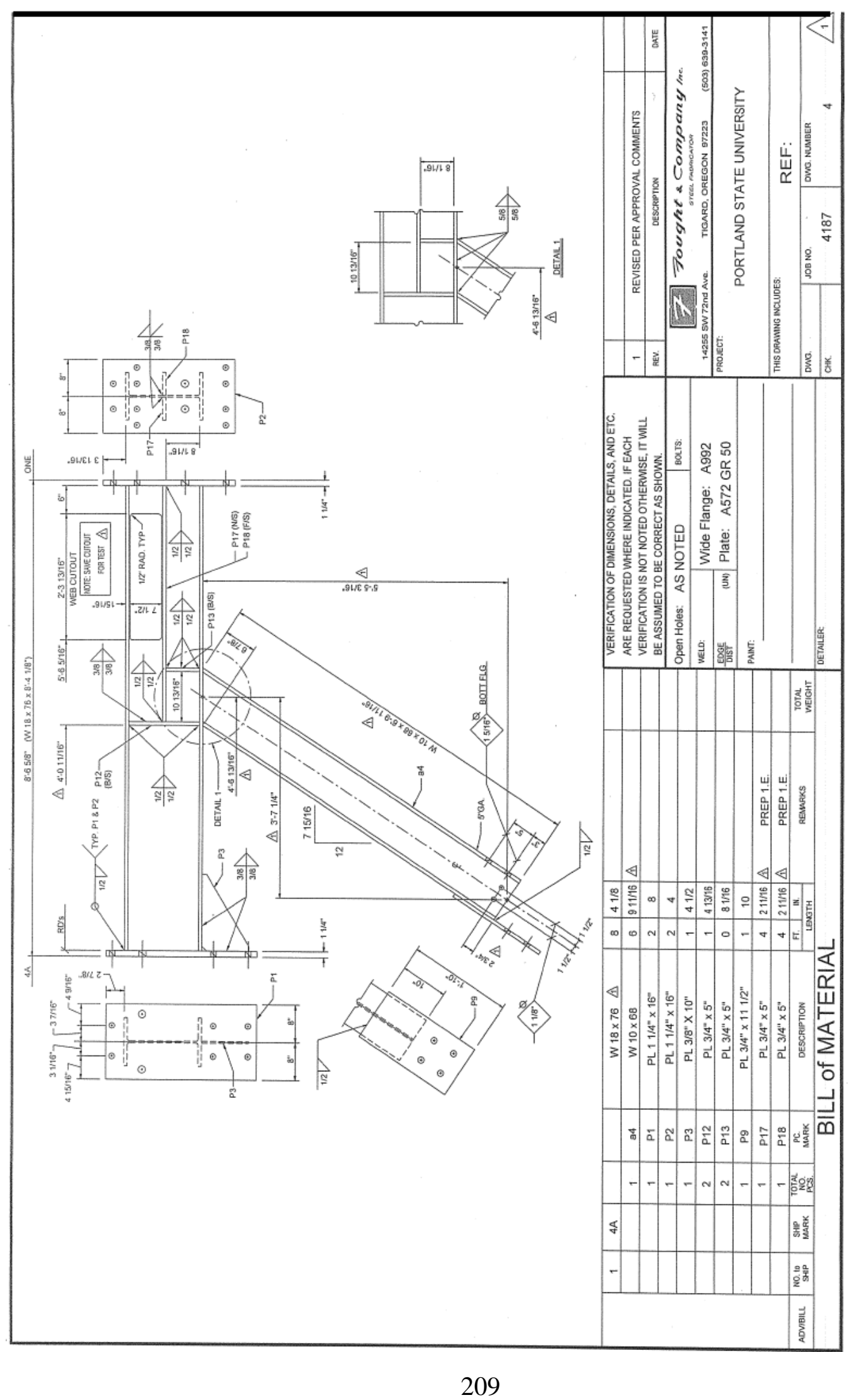




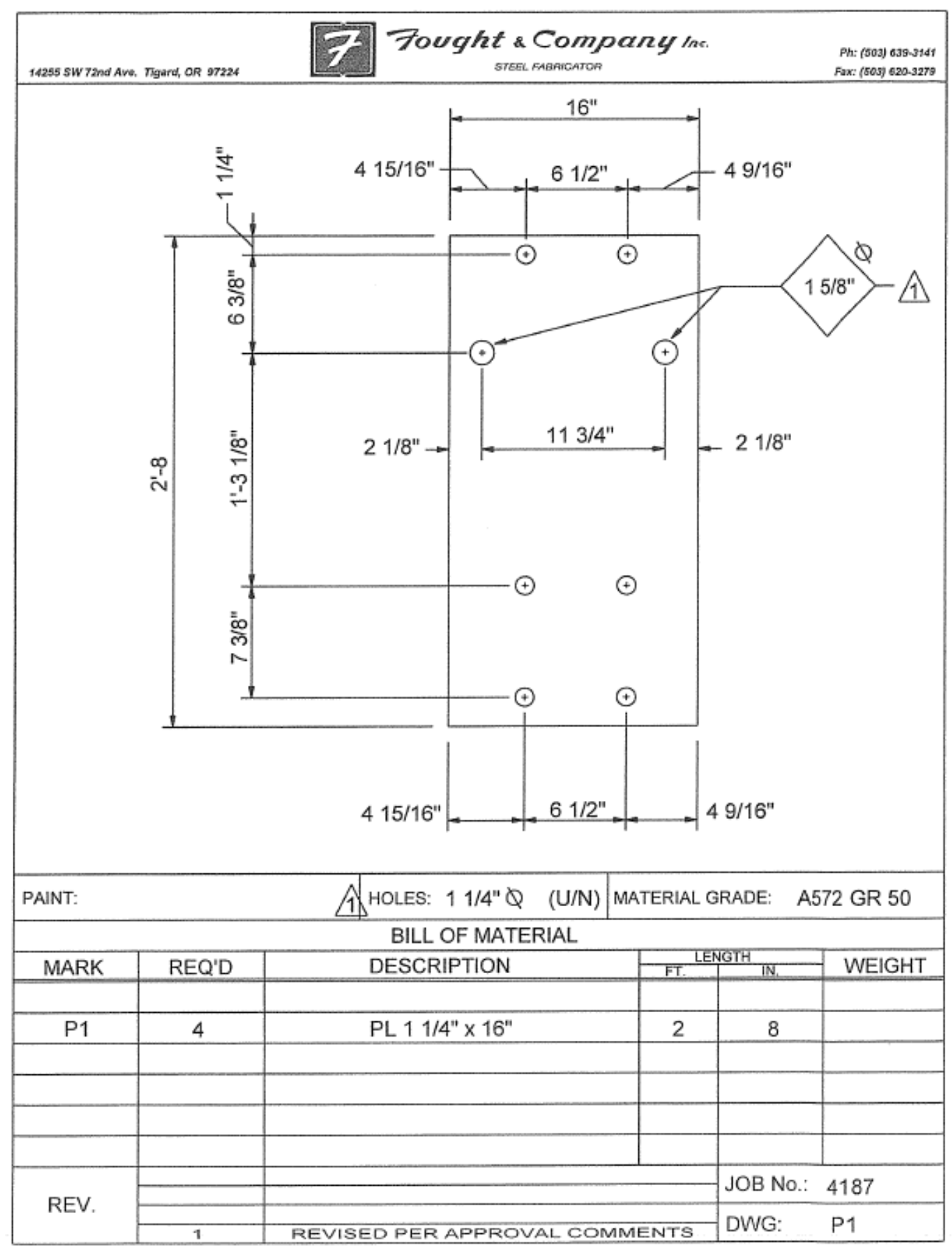




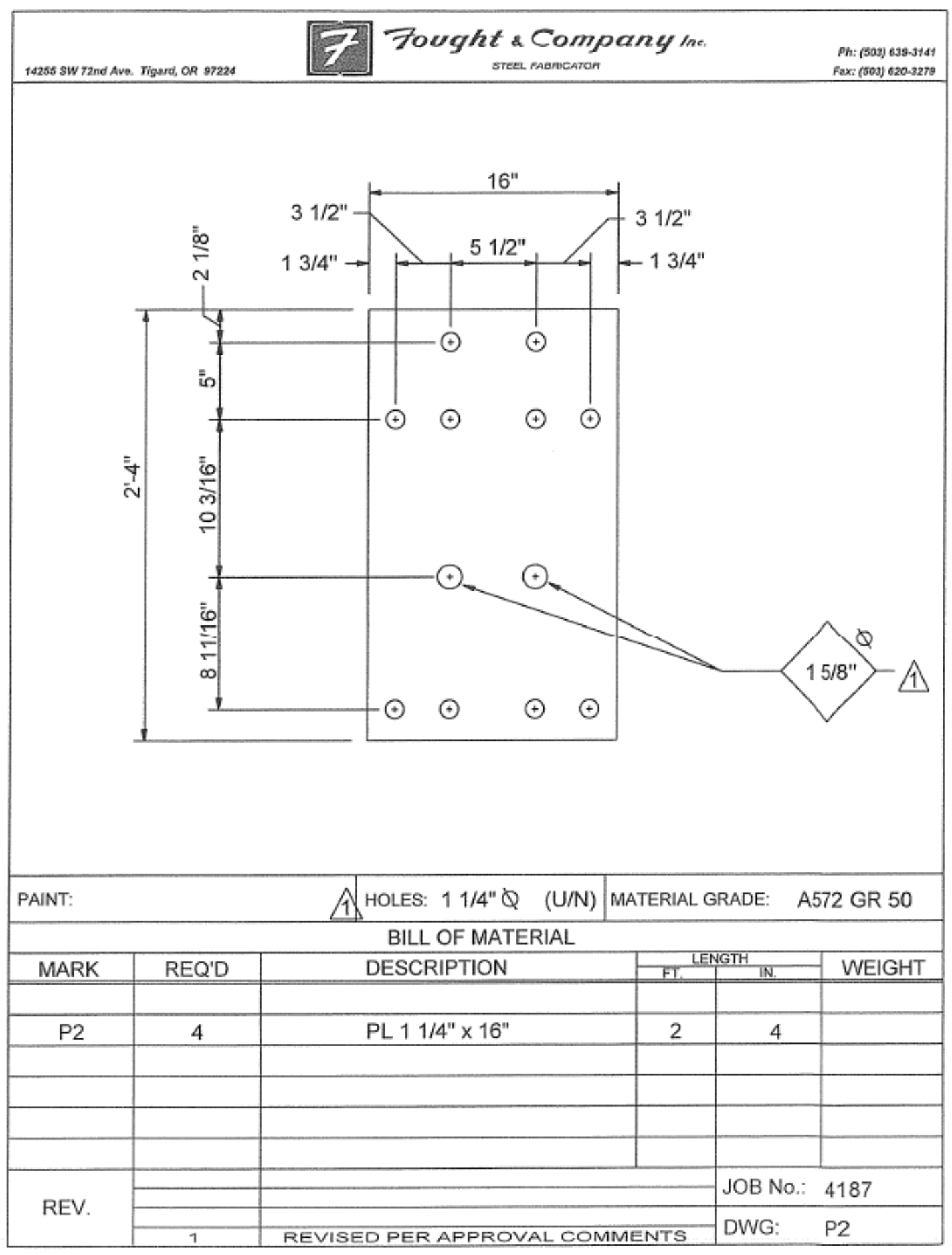




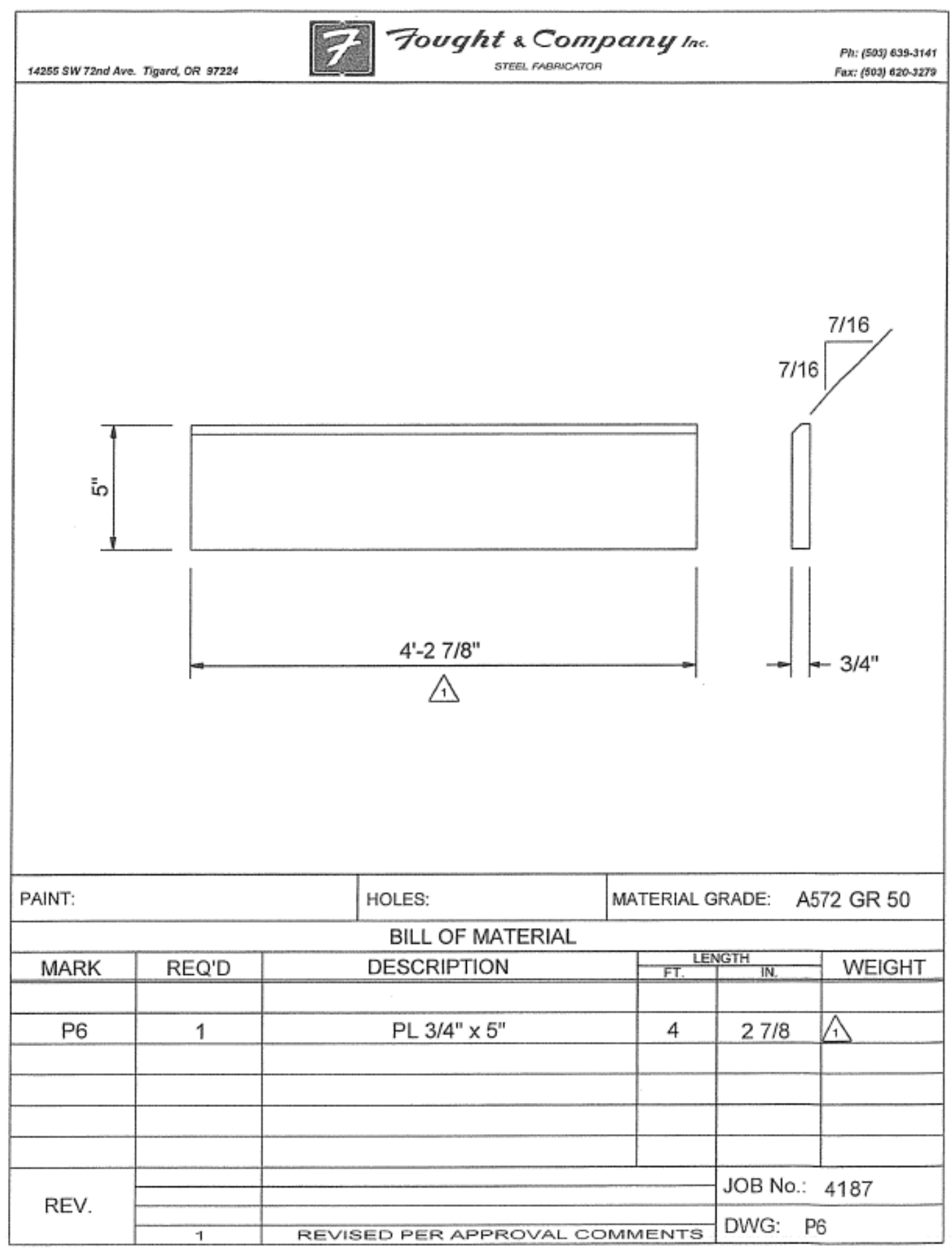




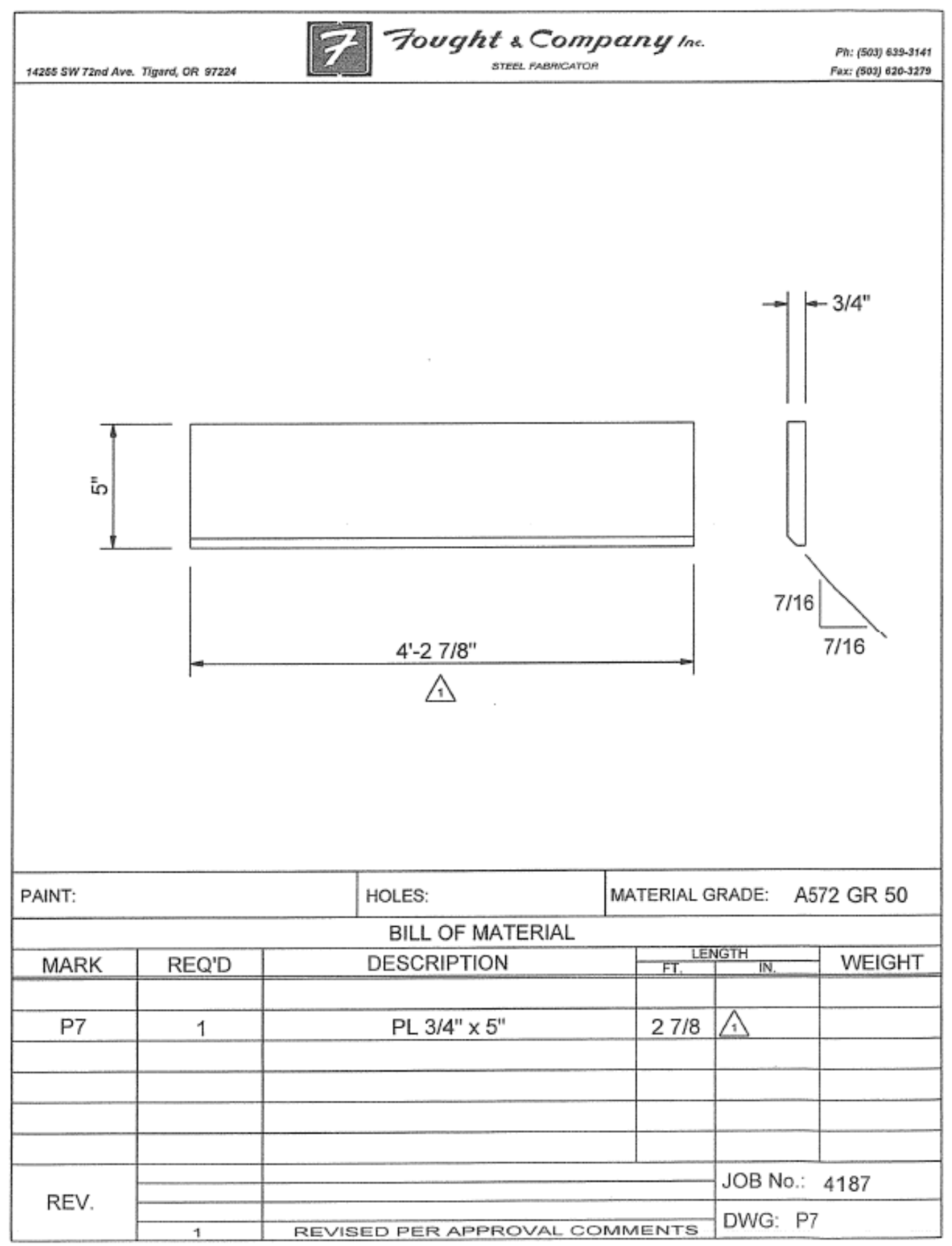




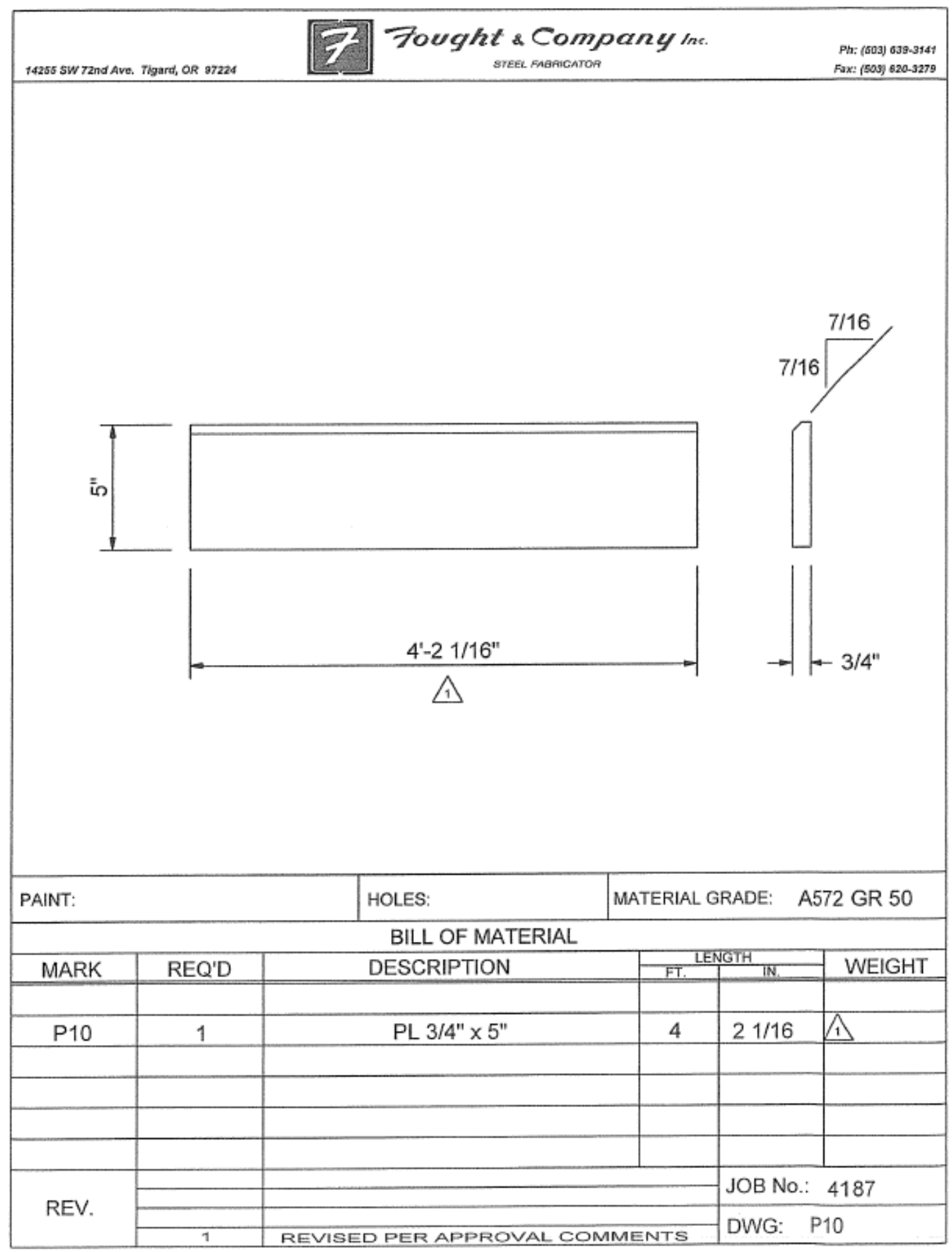




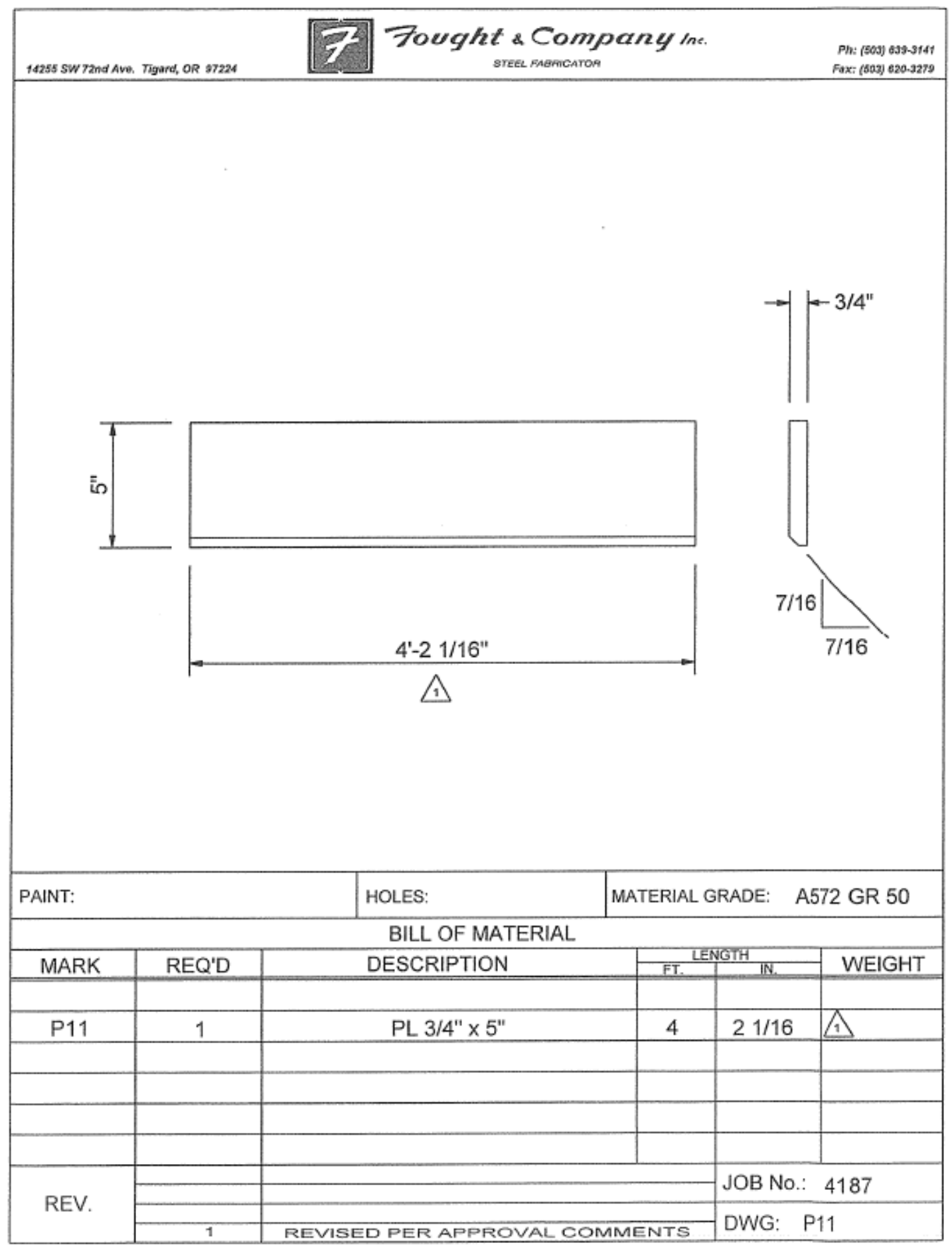




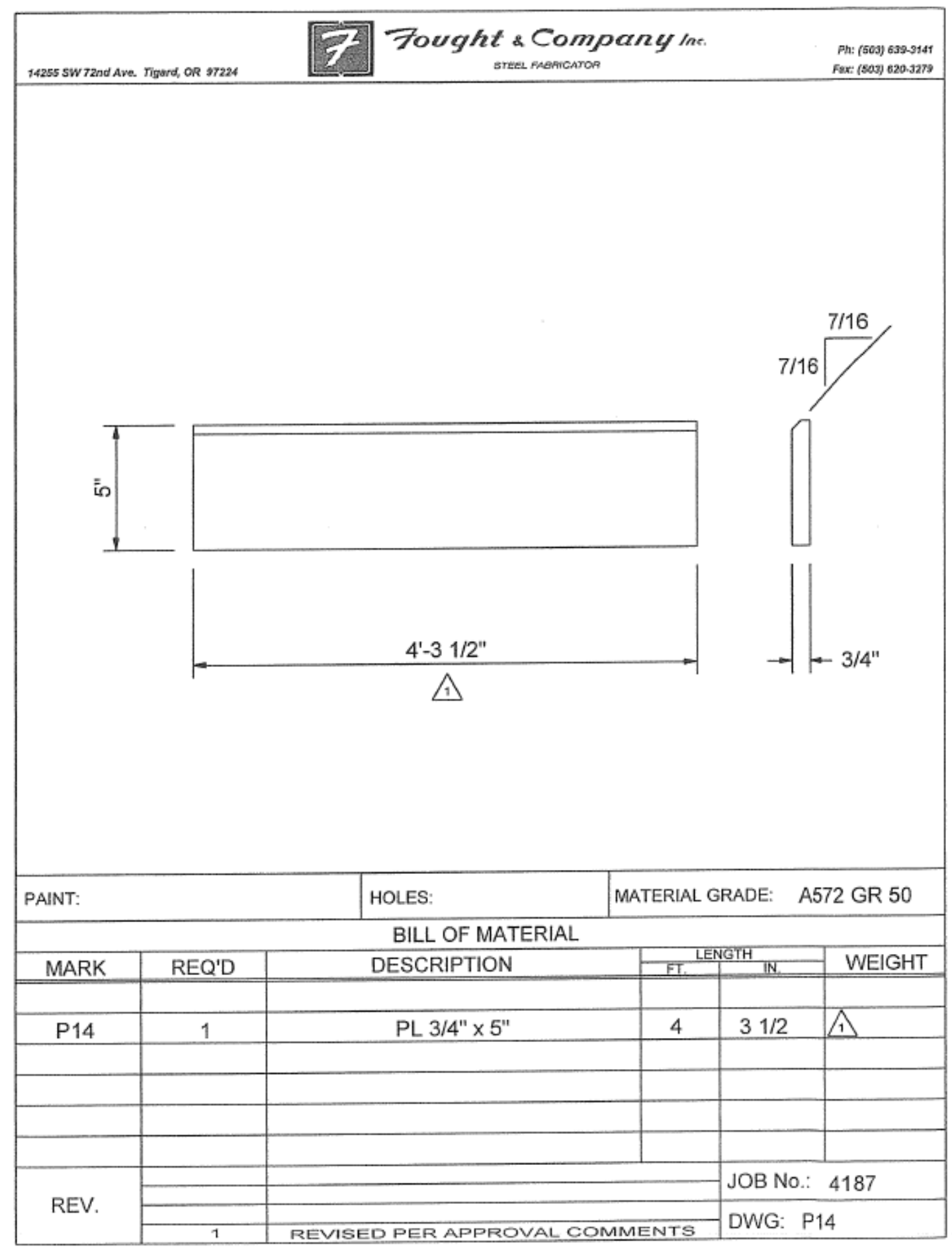




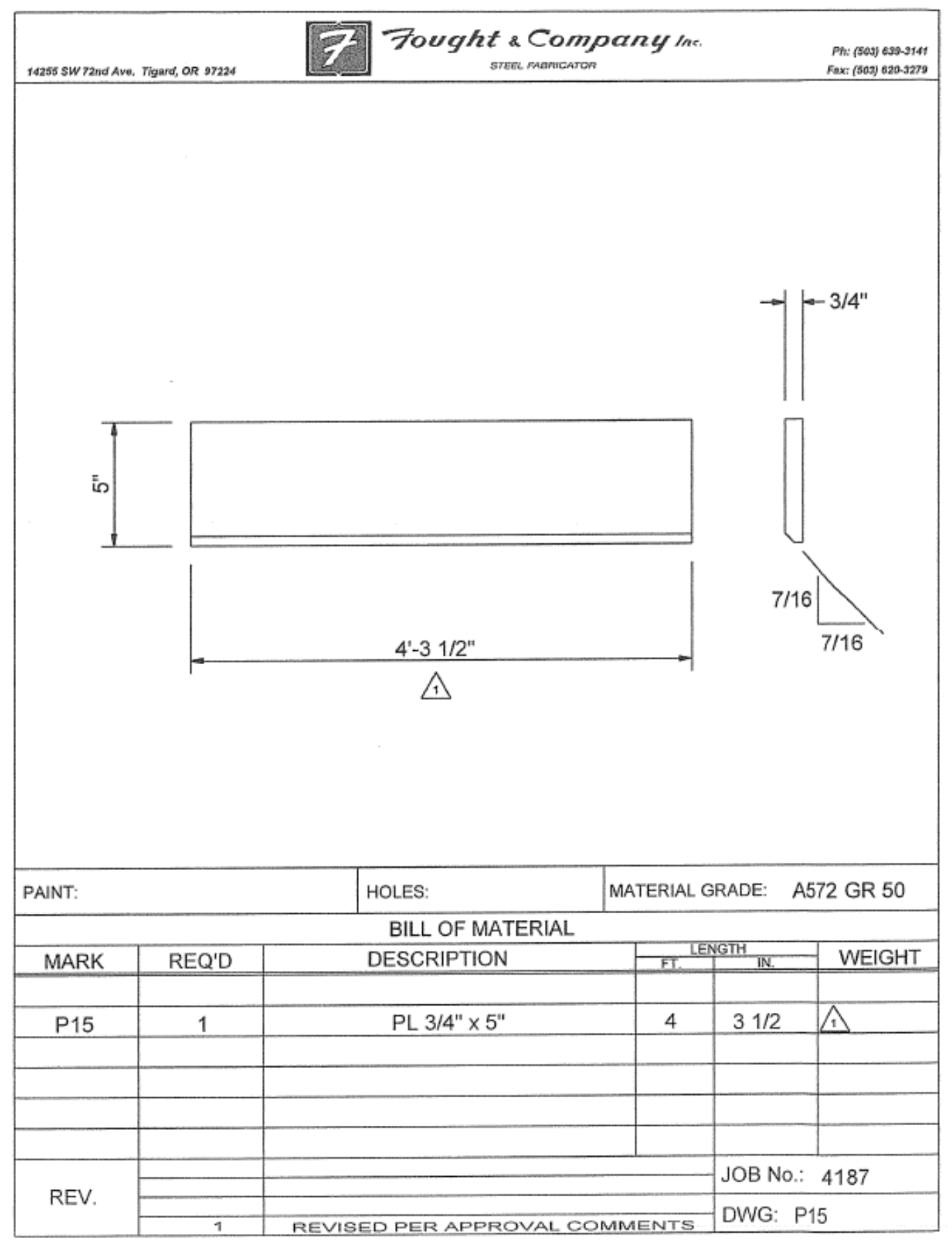




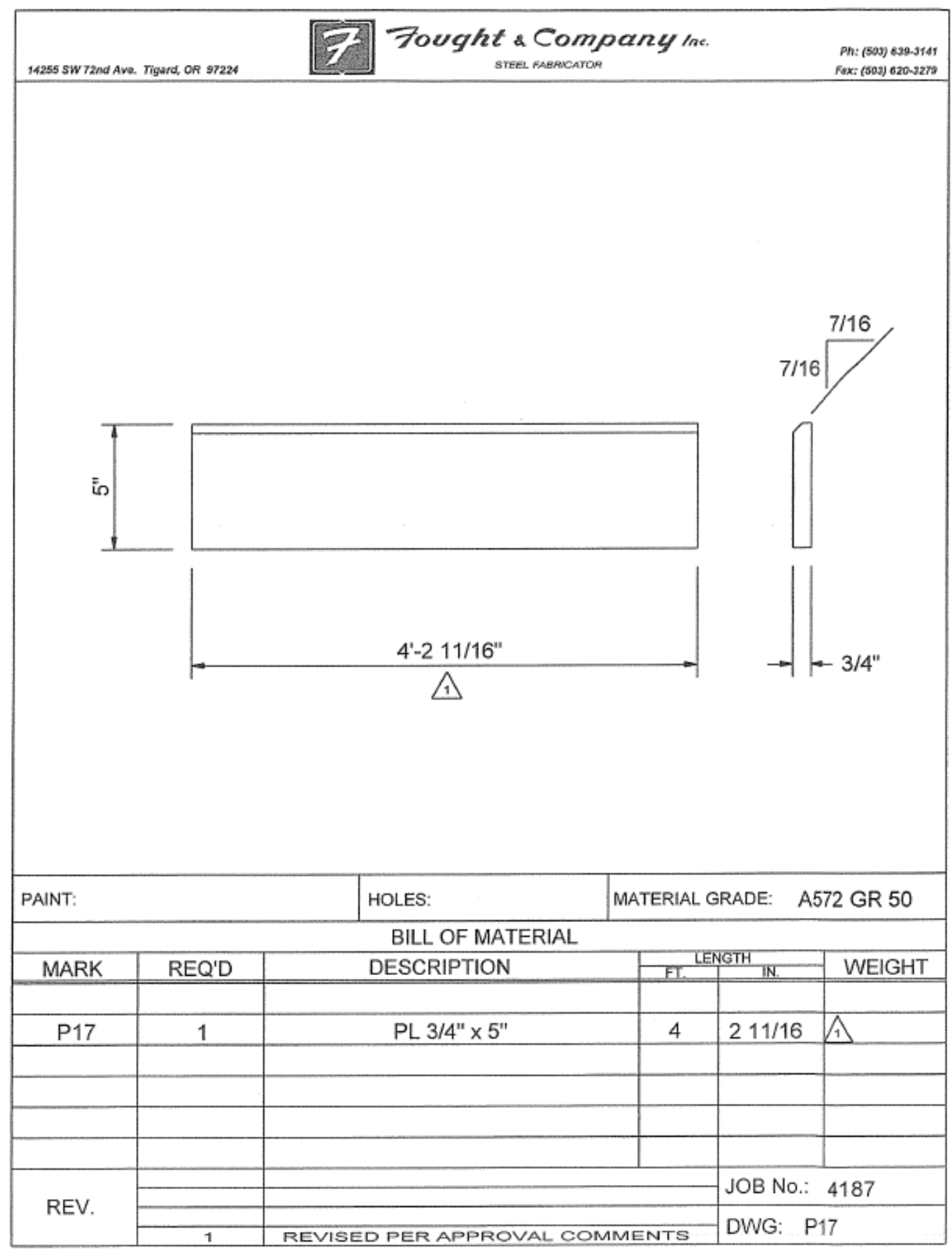




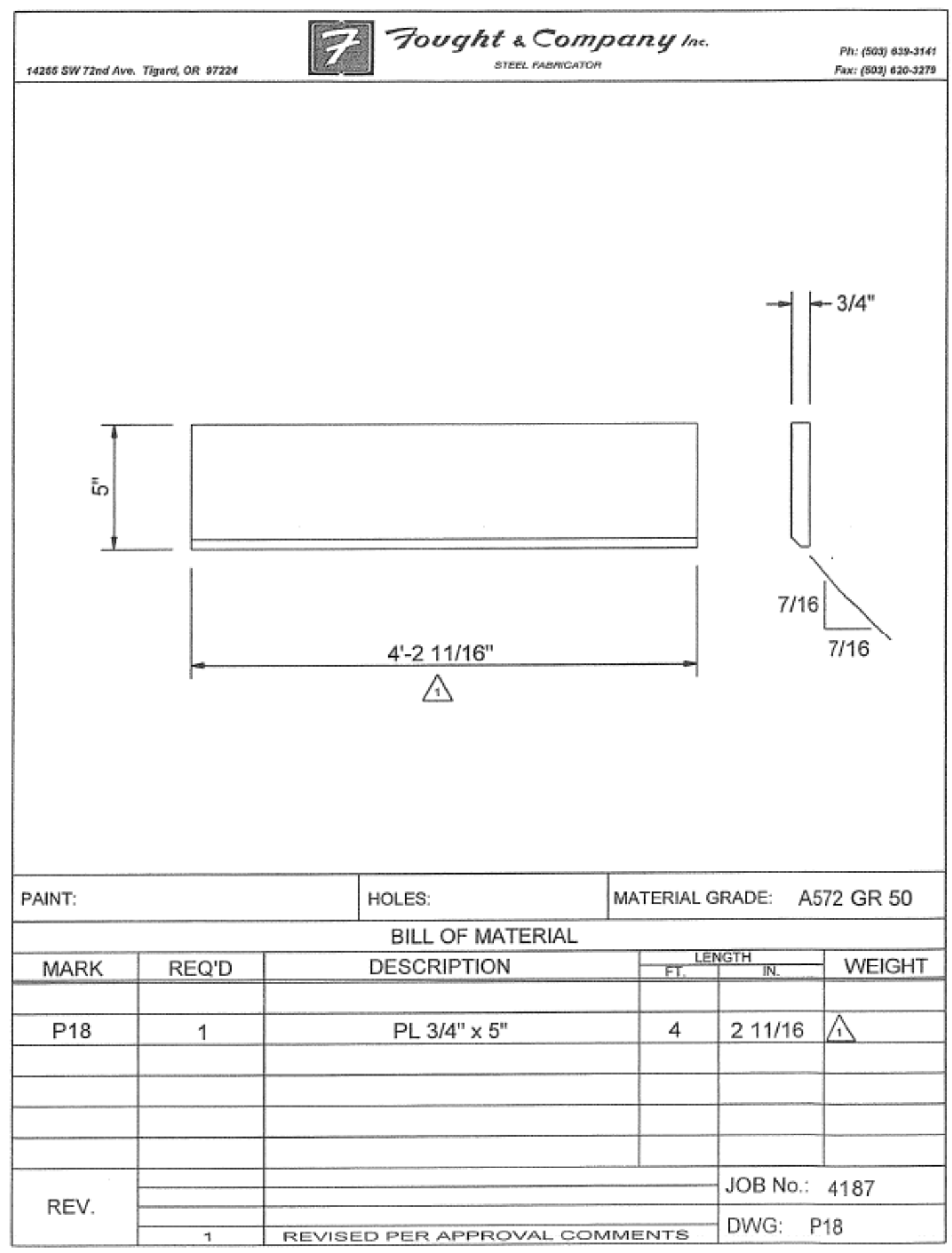




\section{Appendix D. Coupon Test Report}

IMR TEST LABS

A Curtiss-Whight Business Unit

www.imrportland.com
5687-A SE International Way Portland, OR 97222 T: 1.503.653.2904 | F: 1.503.653.9591

\section{TEST REPORT}

IMR Report Number 201802297

\section{SUMMARY}

Five samples were received for mechanical testing.

February 12, 2018

Hosam Al-Azzawi

Portland State University - iSTAR

ISTAR Lab

Science Research and Teaching

Center (Lower Level)

1719 SW 10 th

Portland, OR 97201

PONumber

Credit Card

Date Received

January 30,2018

Material

Steel
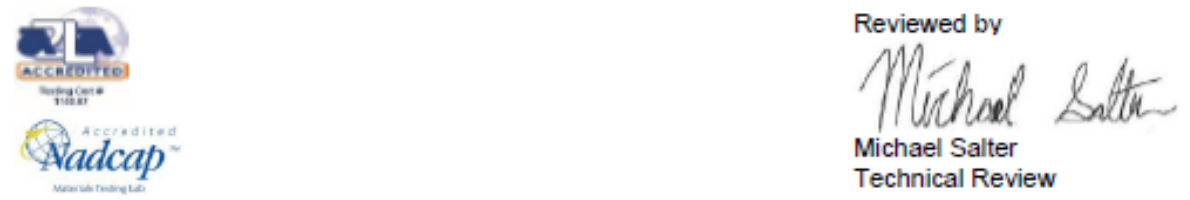

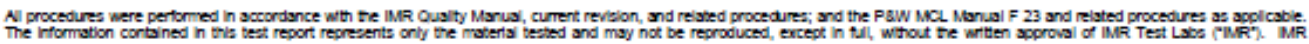

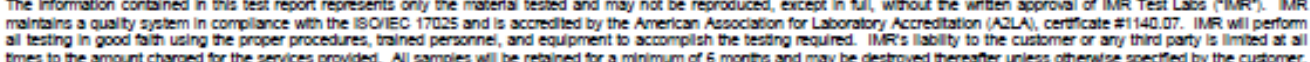

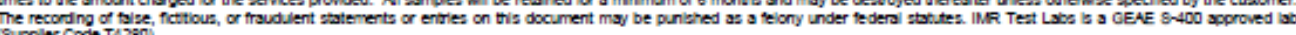
(Buppler Code T4280 
IMR Test Labs - Portland • 5687-A SE International Way • Portland, OR 97222 ROOM TEMPERATURE TENSILE TEST

\begin{tabular}{|c|c|c|c|c|c|c|}
\hline $\begin{array}{c}\text { Laboratory } \\
\text { Number }\end{array}$ & $\begin{array}{c}\text { Tensile } \\
\text { Strength } \\
(\mathbf{k s i})\end{array}$ & $\begin{array}{c}\text { Yield } \\
\text { Strength } \\
(\mathrm{ksi})\end{array}$ & $\begin{array}{c}\text { Elongation } \\
\text { in 2" } \\
(\%)\end{array}$ & $\begin{array}{c}\text { Orig. } \\
\text { Gage } \\
\text { Width } \\
\text { (in.) }\end{array}$ & $\begin{array}{c}\text { Orig. } \\
\text { Gage } \\
\text { Thickness } \\
\text { (in.) }\end{array}$ & Sample ID \\
\hline $02297-1 \mathrm{FTT}$ & 75.7 & 53.9 & 35.5 & 0.504 & 0.738 & Stiffener \\
\hline $02297-2 \mathrm{FTT}$ & 81.3 & 59.5 & 30.5 & 0.502 & 0.403 & W18_Web/(3A) \\
\hline $02297-3 \mathrm{FTT}$ & 77.4 & 57.2 & 35.5 & 0.504 & 0.642 & W18_Flange 3A \\
\hline $02297-4 \mathrm{FTT}$ & 80.0 & 59.4 & 30.5 & 0.505 & 0.392 & W16_Web (1A) \\
\hline $02297-5 \mathrm{FTT}$ & 77.2 & 56.3 & 34.0 & 0.507 & 0.634 & W-16_Flange (1A) \\
\hline
\end{tabular}

Yield strength: $0.2 \%$ offset method

Method(s): ASTM A370-17 